\title{
Evaluating utilization of beta -blockers as secondary prevention for post myocardial infarction in a Medicaid population
}

Ancilla W. Fernandes

West Virginia University

Follow this and additional works at: https://researchrepository.wvu.edu/etd

\section{Recommended Citation}

Fernandes, Ancilla W., "Evaluating utilization of beta -blockers as secondary prevention for post myocardial infarction in a Medicaid population" (2003). Graduate Theses, Dissertations, and Problem Reports. 1863.

https://researchrepository.wvu.edu/etd/1863

This Dissertation is protected by copyright and/or related rights. It has been brought to you by the The Research Repository @ WVU with permission from the rights-holder(s). You are free to use this Dissertation in any way that is permitted by the copyright and related rights legislation that applies to your use. For other uses you must obtain permission from the rights-holder(s) directly, unless additional rights are indicated by a Creative Commons license in the record and/ or on the work itself. This Dissertation has been accepted for inclusion in WVU Graduate Theses, Dissertations, and Problem Reports collection by an authorized administrator of The Research Repository @ WVU.

For more information, please contact researchrepository@mail.wvu.edu. 
Evaluating Utilization of Beta-Blockers as Secondary Prevention for Post Myocardial Infarction in a Medicaid Population

Ancilla W. Fernandes

Dissertation Submitted to the

School of Pharmacy

at West Virginia University

in partial fulfillment of the requirements for the degree of

Doctor of Philosophy

in

Pharmaceutical Systems and Policy

Suresh S. Madhavan, Ph.D. Chair

Mayur M. Amonkar, Ph.D. Co-Chair

Dawn Dell, PharmD.

Syed Islam, Ph.D.

Virginia Scott, Ph.D.

Department of Pharmaceutical Systems and Policy

Morgantown, West Virginia

2003

Keywords: Medicaid, AHA/ACC guidelines, inappropriate prescribing, and utilization Copyright 2003 Ancilla W. Fernandes 


\section{ABSTRACT \\ Evaluating Utilization of Beta-Blockers as Secondary Prevention for Post Myocardial Infarction in a Medicaid Population}

\section{Ancilla W. Fernandes}

Acute myocardial infarction (AMI) is associated with high mortality and costs to the US healthcare system. Beta-blockers are known to reduce mortality and re-infarction rates when used for long-term prevention following an AMI. They are recommended by the American College of Cardiology (ACC)/American Heart Association (AHA) guidelines in post-AMI patients. However, this therapy is both underused (error of omission in eligible patients) and misused (error of commission in ineligible patients). This study involved two phases. Phase one included evaluating utilization of beta-blockers in a Medicaid population and determining the effect of their utilization on patient outcomes such as mortality, morbidity, utilization of healthcare services and expenditures. Phase two involved determining the association of physician-related factors such as knowledge of contraindications, willingness to prescribe, physician demographics and physician practice characteristics on their beta-blocker prescribing behavior. Phase one of the study revealed $37 \%$ inappropriate (misuse and underuse) utilization. During the 12-month follow-up after the incident AMI the appropriately prescribed group had a significantly lower all-cause mortality and lower, but insignificant, cardiac mortality compared to the inappropriately prescribed group. The appropriately prescribed group had significantly higher cardiovascular morbidity and higher utilization in the follow-up period. However, there were indications that the appropriate group was more severely ill as compared to the inappropriate group. Thus, the increase in morbidity and utilization could be due to patient severity rather than appropriate therapy. In phase two, a survey was mailed to 1,019 physicians involved in post-AMI care in WV, of which $261(25.61 \%)$ responded. Physician knowledge of contraindications was not associated with their self-reported beta-blocker prescribing behavior. Physicians' willingness to prescribe was positively associated with their beta-blocker prescribing behavior. Younger age and affiliation with a larger hospital were associated with better beta-blocker prescribing behavior. Multivariate analysis including knowledge, willingness to prescribe, demographics and practice characteristics revealed that willingness to prescribe was the only significant predictor of their beta-blocker prescribing behavior. Findings of this phase indicated a profile of general specialty/family practice physician, older in age, non-university or nonhospital affiliated, and attached to a smaller hospital as the target for interventions to improve beta-blocker prescribing behavior. 
For Mummy and Daddy 


\section{ACKNOWLEDGEMENT}

I would like to take this opportunity to thank the people who made this study possible. Most of all, I would like to express my deepest gratitude to Dr. Suresh Madhavan, my major advisor and Committee Chairperson, for his thoughtful guidance, patience, assistance as well as friendship throughout the course of this study. I would also like to thank Dr. Mayur Amonkar, Co-Chairperson on my Committee. His faith and support helped and aided in building my confidence and developing my research skills. I feel very fortunate to have had the guidance from both of them.

I want to thank Dr. Dawn Bell for providing her clinical expertise throughout the course of this study. Gratitude is expressed to Dr. Dr. Syed Islam and Dr. Ginger Scott for their time and advice while serving as advisory members on my committee. Their suggestions and guidance contributed to the success of this study. I would also like to extend my thanks to Dr. Kimberly Horn and the entire staff at ODAIS for their invaluable friendship and support during my dissertation.

Next, I would like to express appreciation to the staff of school of pharmacy, especially Marion and Debra. I will always be grateful for their prompt support. Further, I am grateful to my fellow graduate students for their help, cooperation and encouragement.

Finally, my sincere gratitude to those who deserve the greatest credit for all that I have accomplished - my parents for giving me strength and inspiration at every step in life, to my brother for his loving support and my friends who gave me encouragement through these years of graduate school. I want them to know that I couldn't have made it without them. 


\title{
TABLE OF CONTENTS
}

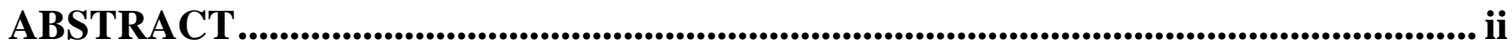

ACKNOWLEDGEMENT ...................................................................................................iv

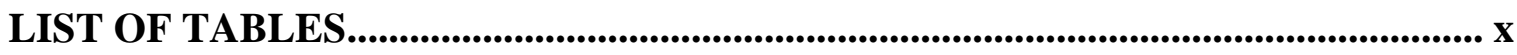

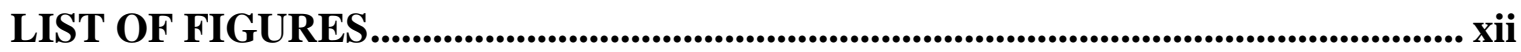

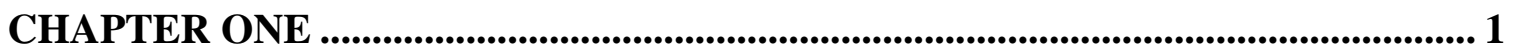

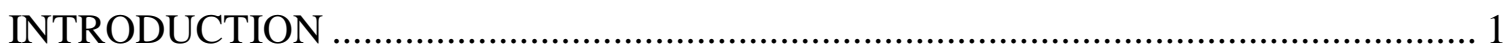

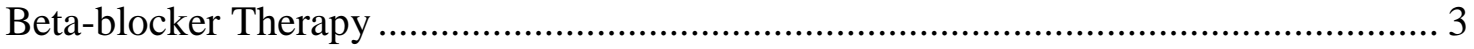

Evidence of Inappropriate Prescribing ……………….............................................. 5

Consequences of Inappropriate Prescribing …………….......................................... 7

Physicians' Role in Drug Prescribing................................................................. 9

STATEMENT OF PROBLEM …………………………….................................... 12

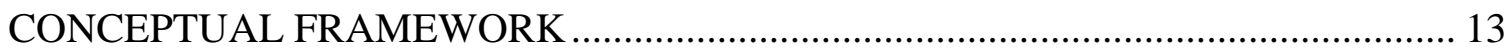

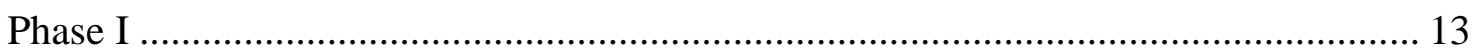

Selection of Medicaid Population …………………………................................ 13

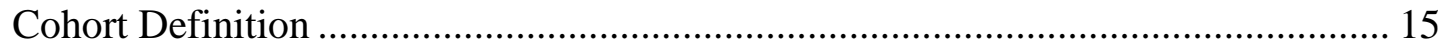

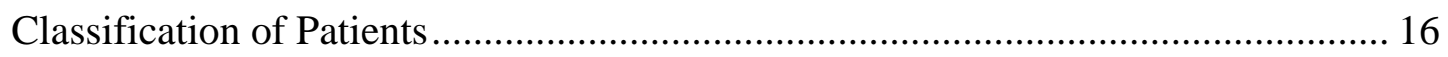

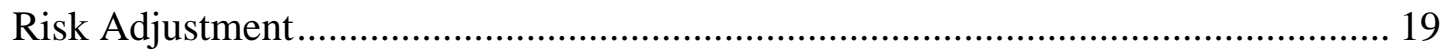

Evaluation of Outcomes ..................................................................................... 22

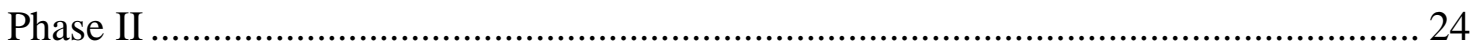

STUDY GOALS, AND RESEARCH QUESTIONS …………………………........ 28

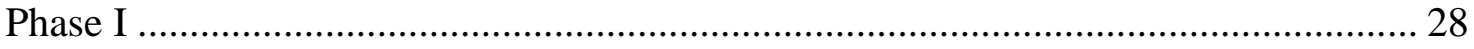

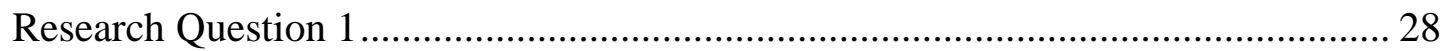

Research Question 2................................................................................. 28

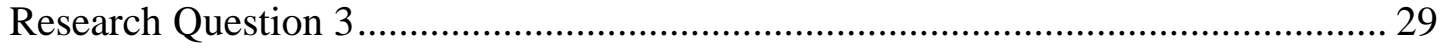




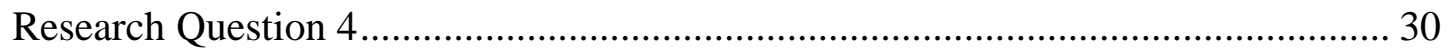

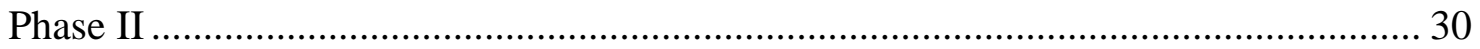

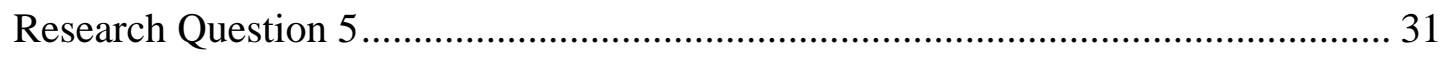

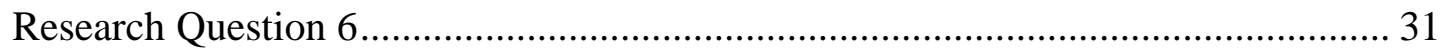

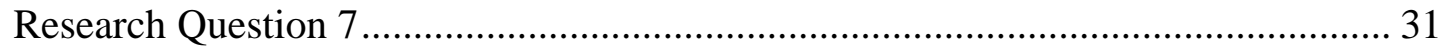

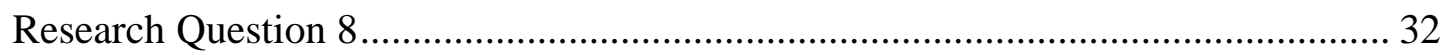

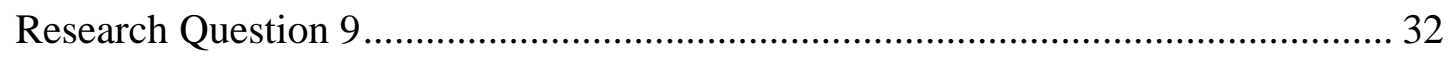

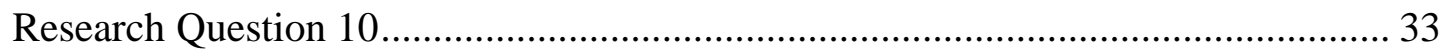

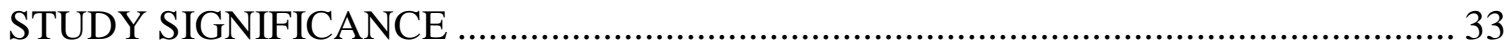

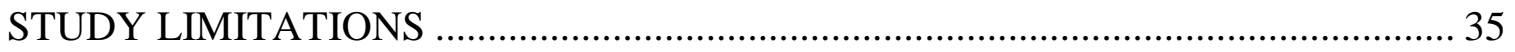

CHAPTER TWO

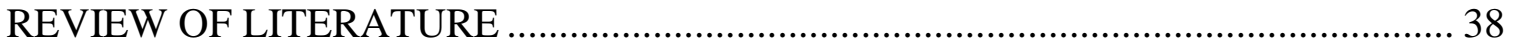

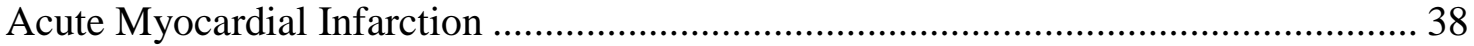

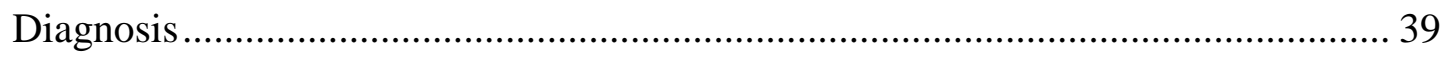

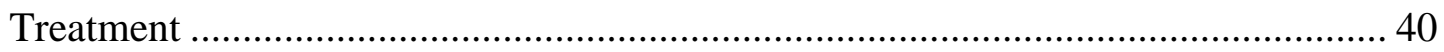

Beta-Blocker Therapy............................................................................................. 42

Effectiveness of Beta-Blockers for Post AMI ........................................................... 44

Recommendation for Beta-Blocker Use for Post AMI ................................................ 53

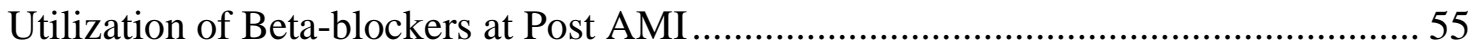

Implications of Inappropriate Utilization of Beta-Blocker Therapy .............................. 61

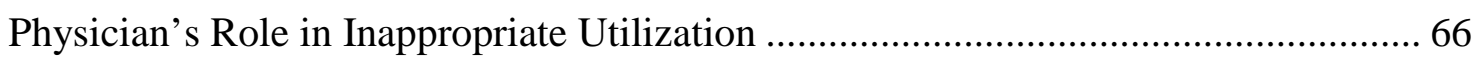

Medicaid Program …………………….................................................................... 69

CHAPTER THREE ......................................................................................................... 72

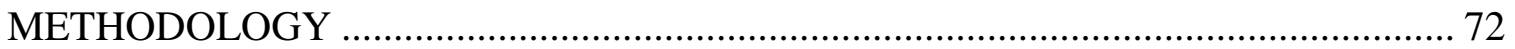

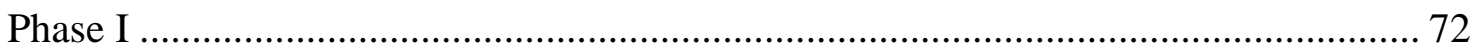

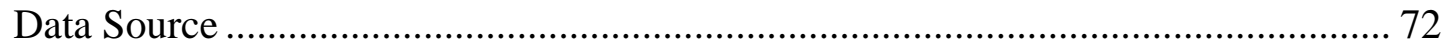

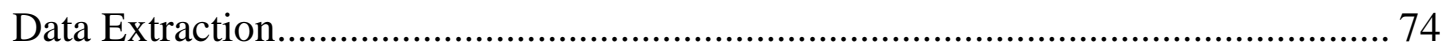

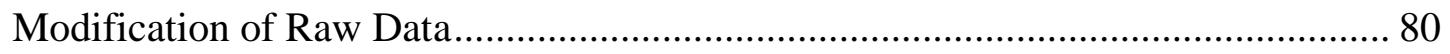




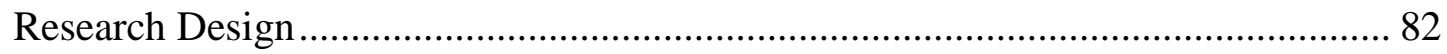

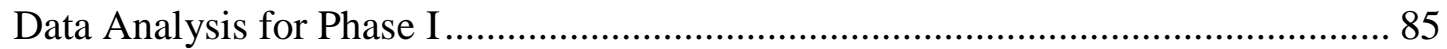

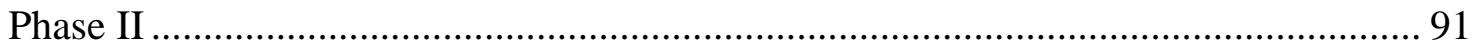

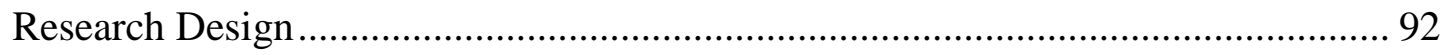

Study Population and Sample Selection …………………..................................... 92

Instrument Development and Content …………………................................... 92

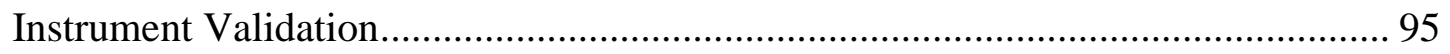

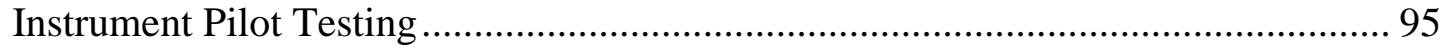

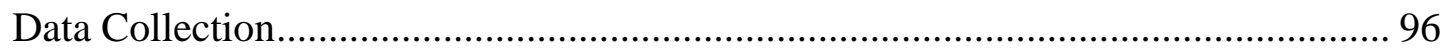

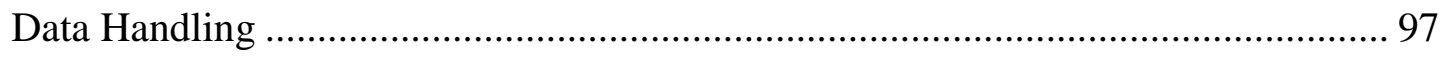

Data Analysis for Phase II................................................................................ 98

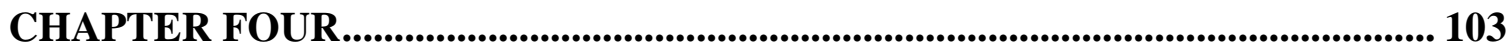

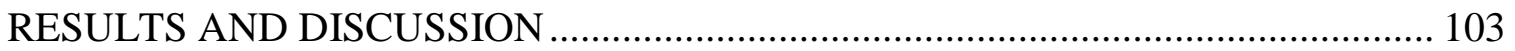

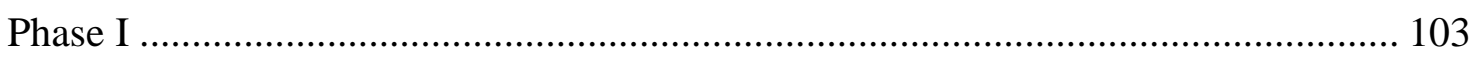

Demographic and Medical History of Patients in Phase I .................................... 106

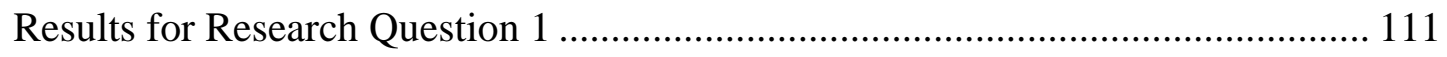

Discussion for Research Question 1 ................................................................ 119

Results for Research Question 2 ..................................................................... 120

Discussion for Research Question 2 .............................................................. 127

Results for Research Question 3 ...................................................................... 128

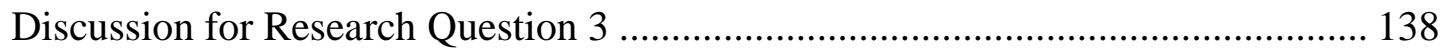

Results for Research Question 4 ........................................................................ 138

Discussion for Research Question 4 ................................................................ 162

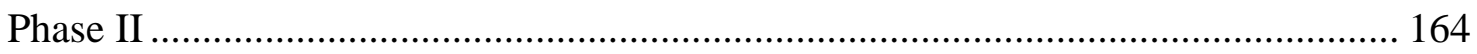

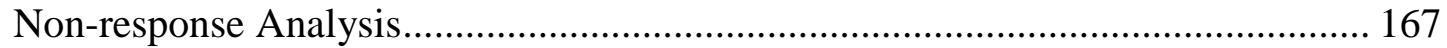

Demographic and Practice Characteristics of Physicians in Phase II ..................... 174

Results for Research Question 5 ...................................................................... 177

Discussion for Research Question 5 ................................................................... 184

Results for Research Question 6 ...................................................................... 184

Discussion for Research Question 6 ................................................................ 191 


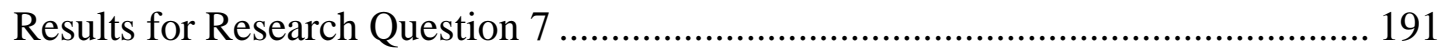

Discussion for Research Question 7 .................................................................. 196

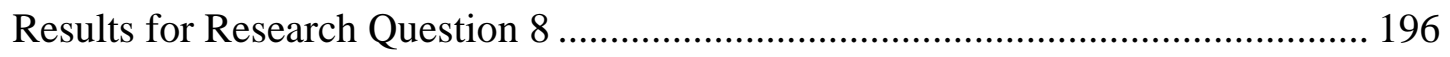

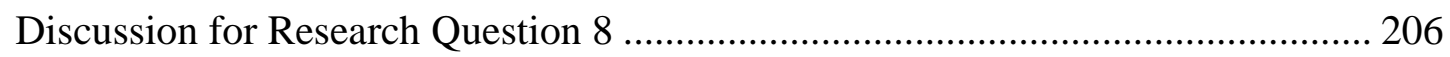

Results for Research Question 9 .......................................................................... 208

Discussion for Research Question 9 ................................................................ 211

Results for Research Question 10 ..................................................................... 214

Discussion for Research Question 10 ............................................................... 217

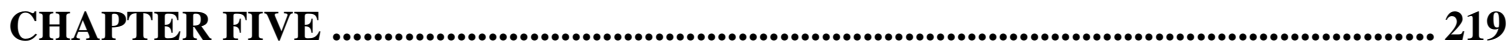

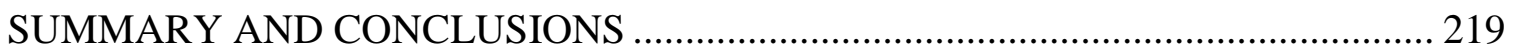

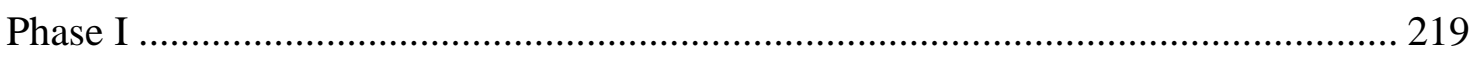

Conclusions for Phase I................................................................................. 220

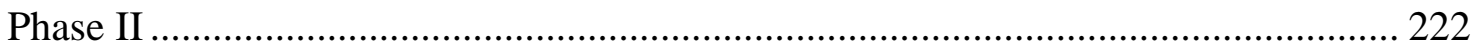

Conclusions for Phase II ................................................................................ 223

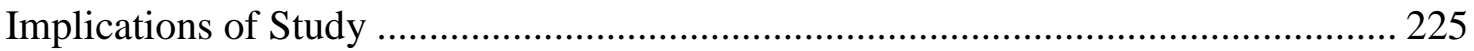

Implication to the Medicaid Program ………………………………................ 226

Implication to Patients................................................................................. 227

Implications to Prescribers …………….......................................................... 227

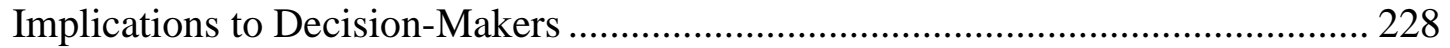

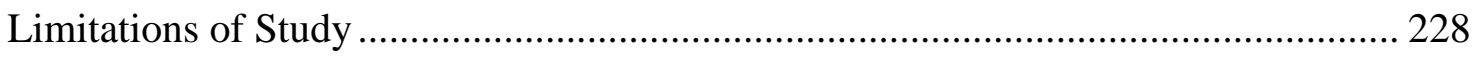

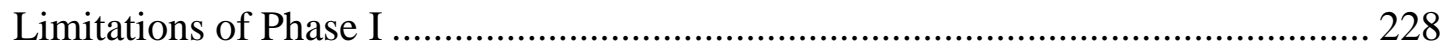

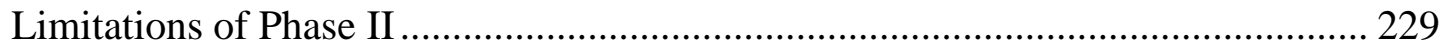

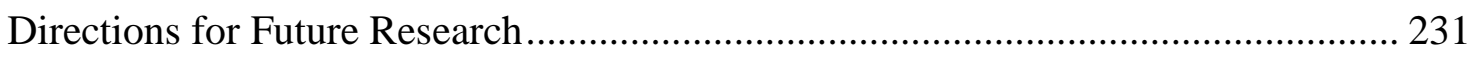

BIBLIOGRAPHY ....................................................................................................................... 233

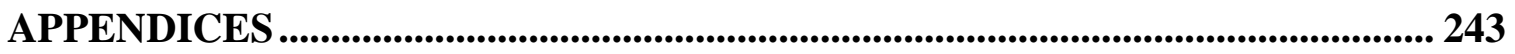

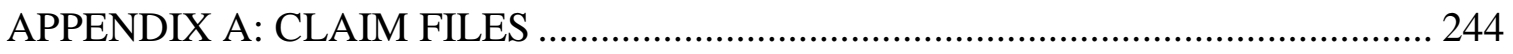

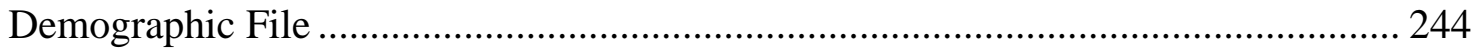

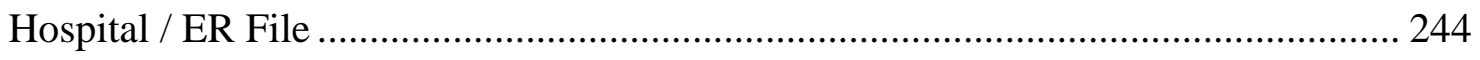




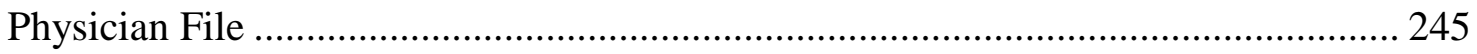

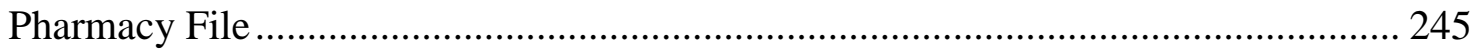

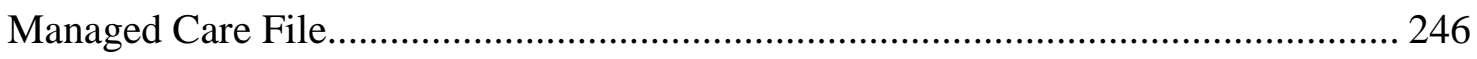

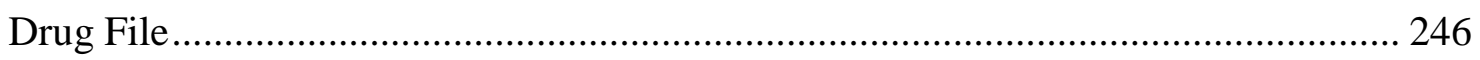

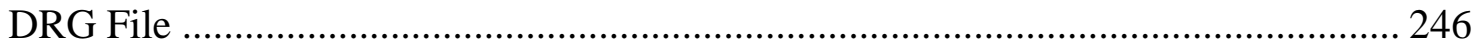

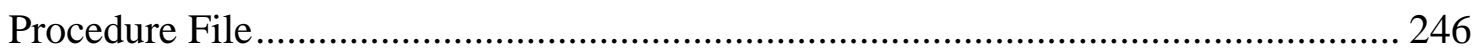

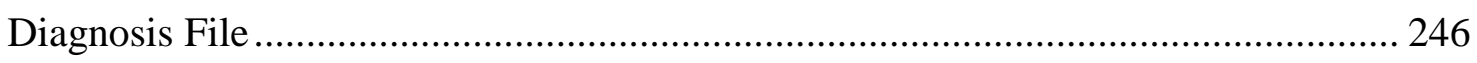

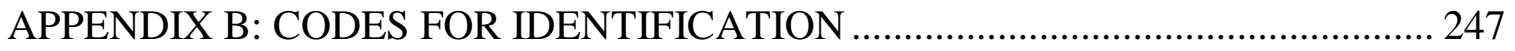

Relative Contraindications to Beta-blocker Therapy Post Acute Myocardial Infarction

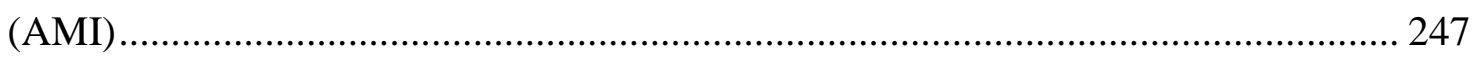

Absolute Contraindications to Beta-blocker Therapy Post AMI............................... 247

Identifying Codes for Other Conditions Utilized in the Study ................................. 248

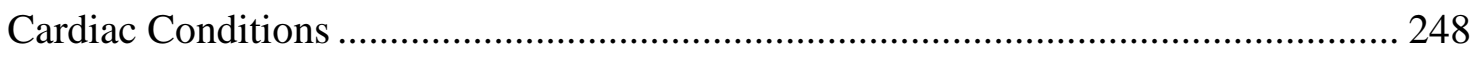

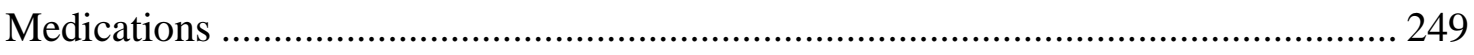

APPENDIX C: FIRST COVER LETTER ............................................................. 251

APPENDIX D: SECOND COVER LETTER …....................................................... 252

APPENDIX E: THIRD COVER LETTER ............................................................ 253

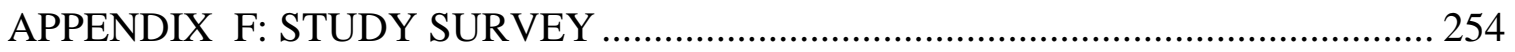

APPENDIX G: NON-RESPONSE SURVEY ..................................................... 259

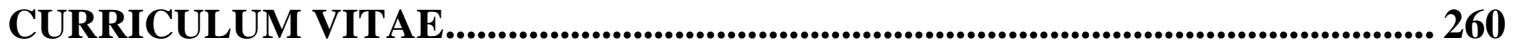




\section{LIST OF TABLES}

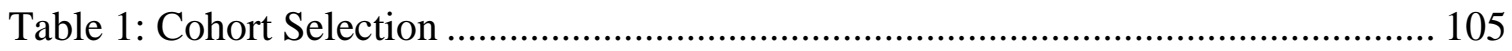

Table 2: Demographic Characteristics of the Final Phase I Study Patients.................... 108

Table 3: Medical History and Other Characteristics......................................................... 109

Table 4: Prescription Patterns for Beta-blocker Use...................................................... 115

Table 5: Univariate Comparisons between the Two Groups ......................................... 116

Table 6: Predictors for Appropriate/Inappropriate Group ............................................. 118

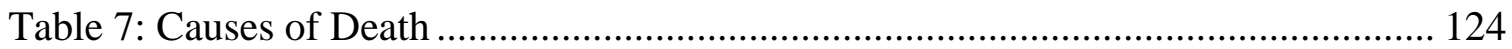

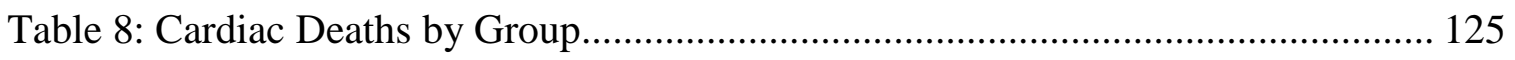

Table 9: Patient Deaths by Group Status ................................................................... 126

Table 10: Cardiovascular Events for Morbidity ............................................................. 131

Table 11: Cardiac Events by Group Status ............................................................. 134

Table 12: Cox-Proportional Regression Model for Time to First Cardiac Event........... 135

Table 13: Cox-Proportional Regression Model for Time to First Cardiac Event using

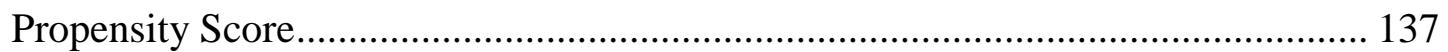

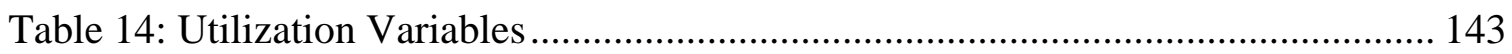

Table 15: Predictors for Number of Hospital Visits due to Cardiac Conditions ............ 144

Table 16: Predictors for Number of Hospital Visits due to Cardiac Conditions using

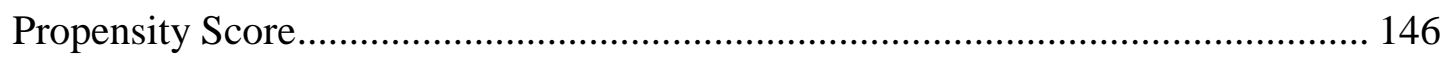

Table 17: Predictors for Number of ER Visits due to Cardiac Conditions...................... 147

Table 18: Predictors for Number of ER Visits due to Cardiac Conditions using Propensity

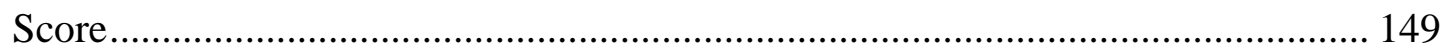

Table 19: Predictors for Number of Physician Visits due to Cardiac Conditions .......... 150

Table 20: Predictors for Number of Physician Visits due to Cardiac Conditions using

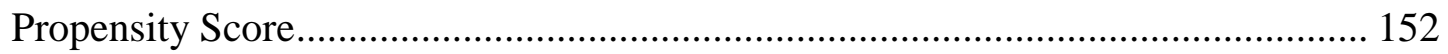

Table 21: Predictors for Length of Stay due to Cardiac Conditions............................... 153

Table 22: Predictors for Length of Stay due to Cardiac Conditions using Propensity Score

Table 23: Predictors for Log Charges due to Cardiac Conditions ................................. 156

Table 24: Predictors for Log Charges due to Cardiac Conditions Using Propensity Score 
Table 25: Predictors for Log Reimbursed Amounts due to Cardiac Conditions .....

Table 26: Predictors for Log Reimbursed Amounts due to Cardiac Conditions using Propensity Score.

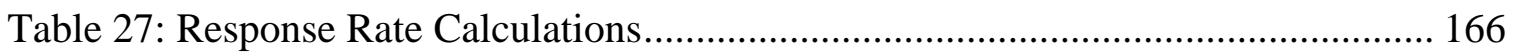

Table 28: Reasons for Not Responding to the Study Survey...................................... 169

Table 29: Demographic and Practice Characteristics of Respondents of the Non-response Survey 170

Table 30: Analysis of Non-response Bias for Demographic and Practice Characteristics

Table 31: Demographic and Practice Characteristics of Respondents ........................ 175

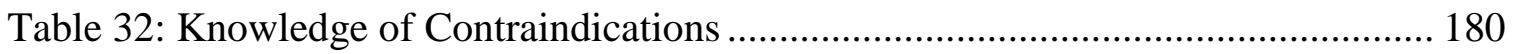

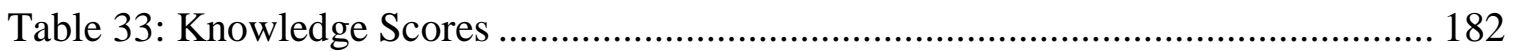

Table 34: Correlation Matrix for Knowledge of Contraindications ............................ 183

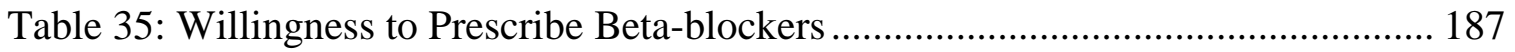

Table 36: Willingness to Prescribe Scores................................................................. 189

Table 37: Correlation Matrix for Willingness to Prescribe ........................................ 190

Table 38: Prescribing Behavior and Demographic Characteristics, Part 1................... 193

Table 39: Prescribing Behavior and Demographic Characteristics, Part 2................... 195

Table 40: Knowledge of Contraindications and Demographic Characteristics, Part 1 .. 200

Table 41: Knowledge of Contraindications and Demographic Characteristics, Part 2 .. 202

Table 42: Willingness to Prescribe and Demographic Characteristics, Part 1 .............. 203

Table 43: Willingness to Prescribe and Demographic Characteristics, Part 2 ............. 205

Table 44: Predictors of Prescribing Percent/Behavior................................................. 210

Table 45: Reasons for Not Prescribing Beta-blockers in Post-AMI Patients ................ 215 


\section{LIST OF FIGURES}

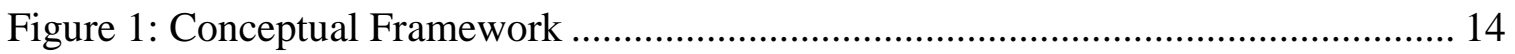

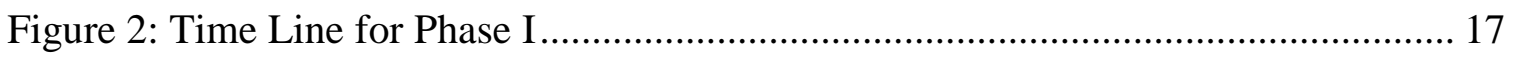

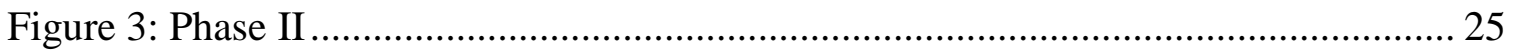

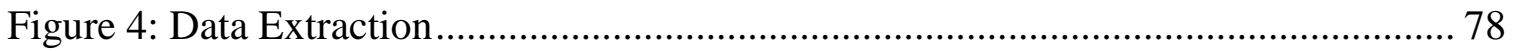

Figure 5: Quasi-experimental Longitudinal Research Design ..................................... 84

Figure 6: Appropriate/Inappropriate Use of Beta-blockers ........................................ 114

Figure 7: Kaplan-Meier Survival Curves (all-cause deaths) for Appropriate/Inappropriate

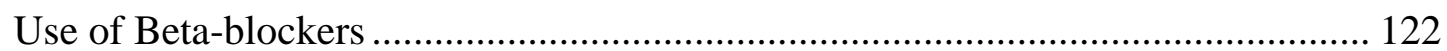

Figure 8: Kaplan-Meier Survival Curves (cardiac deaths) for Appropriate/Inappropriate Use of Beta-blockers 123

Figure 9: Kaplan-Meier Survival Morbidity Curves (time to first cardiovascular hospitalization) for Appropriate/Inappropriate Use of Beta-blockers.

Figure 10: Kaplan-Meier Survival Morbidity Curves (time to recurrent AMI) for Appropriate/Inappropriate Use of Beta-blockers

Figure 11: Summary of Univariate and Multivariate Findings. 


\section{CHAPTER ONE}

\section{INTRODUCTION}

Acute Myocardial Infarction (AMI), also known as "heart attack" is defined as the death of heart muscle due to loss of blood supply (Bellenir, 2000). Coronary artery disease $(\mathrm{CAD})$ is the primary underlying process that leads to an AMI (Stringer \& Lopez, 1999). It is the number one killer among both men and women in the United States (Stringer \& Lopez, 1999). Each year approximately 900,000 people experience an AMI. Apart from being a serious health issue, coronary disease is also responsible for severe economic losses in the United States. For instance, in 1997, the financial consequence due to coronary artery disease to the U.S. healthcare system was estimated to be approximately $\$ 91$ billion (Stringer \& Lopez, 1999). Thus, therapeutic interventions that reduce mortality and improve morbidity, as well as primary and secondary prevention strategies, could have a significant impact on the US healthcare system.

Of the 900,000 people who suffer from an AMI every year, over 800,000 people survive (Bradford, Chen \& Krumholz, 1999). Therapy with beta-blockers has shown to be an effective means of reducing mortality following a heart attack (Bradford, et al., 1999). Prophylaxis with beta-blockers after the onset of an AMI has been reported to be effective since the 1960s (Snow, 1965). Thousands of patients have participated in randomized trials which have demonstrated that cardiovascular mortality and reinfarction rates decrease with beta-blocker use following an AMI (Bradford, et al., 1999). There is also strong evidence that beta-blockers are safe and effective therapies. However, there are certain conditions, such as bradycardia, hypotension and 
atrioventricular (AV) block in patients where there is evidence that therapy is not useful or may be harmful [American College of Cardiology (ACC)/American Heart Association (AHA), 1996]. The national cardiology consensus committees have acknowledged this fact by publishing recommendations for its use in patients to whom it is beneficial and listing those conditions, in the presence of which, this therapy should not be used (ACC/AHA, 1996).

Despite the benefits of beta-blocker therapy and the established guidelines for proper use, they are not prescribed appropriately in patients after an AMI (Bradford, et al., 1999). Many patients who are eligible and should receive prescription for betablocker therapy at discharge do not get a prescription (error of omission or under use). While it is not as well documented, there are a few reports that suggest that sometimes patients who have contraindications and are not eligible for therapy receive a prescription for beta-blockers (error of commission or misuse) (Brand, Newcomer, Freiburger, \& Tian, 1995). This inappropriate prescribing of a useful therapeutic agent can affect patient outcomes such as morbidity, mortality, and increase healthcare utilization and costs. Thus, this study will assess the effects of inappropriate prescribing of betablockers for secondary prevention following an AMI. This study will also assess practitioner knowledge and willingness to prescribe long-term beta-blocker therapy in post AMI patients. The following sections will concentrate on beta-blocker therapy, evidence of its inappropriate prescribing, consequences of inappropriate prescribing, and the physician's role in drug prescribing. 


\section{Beta-blocker Therapy}

By definition, beta-blockers block beta-receptors (Khan, 2000). Structurally they resemble catecholamines. They competitively inhibit beta-receptors depending on the ratio of their concentration to catecholamine concentration. When given to an AMI patient, they are known to rapidly reduce systolic blood pressure, by reducing cardiac output (Plosker \& Clissold, 1992). They are also known to reduce myocardial oxygen demand. These beneficial effects are believed to decrease the incidence of cerebrovascular complications and new myocardial infarctions, thus providing clinical efficacy. Beta-blockers also have certain adverse effects such as fatigue, sexual dysfunction, nightmares and difficulty to detect hypoglycemia in diabetes.

Numerous studies have provided evidence that beta-blocker therapy is effective following an AMI (Bradford, et al., 1999). In a meta-analysis of 65 studies, Yusuf et al. concluded that long-term beta-blocker therapy for perhaps a year or so following discharge after an AMI reduces the odds of death and re-infarction by about 25\% (Yusuf, Peto, Lewis, Collins \& Sleight, 1985). Hjalmarson's review of data from 5 clinical trials reported a $36 \%$ reduction in mortality in two studies, a $26 \%$ reduction in one study, and a $13-14 \%$ reduction in mortality in the other two studies (Hjalmarson, 1988). Goldstein reported that the beneficial effects of oral beta-blocker therapy were maintained for at least six years after myocardial infarction, and discontinuation was associated with accelerated mortality (Goldstein, 1996). He also reported a reduction in cardiovascular mortality, re-infarction rate, and sudden death due to beta-blocker use following an AMI.

Furberg and Byinton examined data from the Beta-Blocker Heart Attack Trial (BHAT) (Furberg \& Byinton, 1983). They examined the difference in outcomes between 
patients in the tails of the distribution of expected mortality risk. They found a relative risk reduction of $40 \%$ in the group receiving beta-blocker agents in this limited set of patients. Olsson et al., analyzed individual patient level data from 5 randomized trials (Olsson, et al., 1992). They found an overall reduction in relative risk of mortality of 19\%. Also, a higher benefit to women (23\%) was observed as compared to men (16\%). The reduction in total mortality was mainly due to a reduction in sudden cardiac deaths. These studies also reported reductions in re-infarction rates, angina pectoris severity, and congestive heart failure severity (Bradford, Chen \& Krumholz, 1999).

The ACC/ AHA relied on the evidence of these beneficial effects to develop guidelines for beta-blocker use for secondary prevention after an AMI (ACC/AHA, 1996 $\&$ 1999). These guidelines suggest that all patients, except those with absolute contraindications to beta-blocker therapy, should begin treatment within a few days of AMI and continue it indefinitely. The absolute contraindications for beta-blocker use are few and include AV block, bradycardia, and hypotension (ACC/AHA, 1996 \& 1999, Philips et al., 2000). However, a variety of conditions are listed as relative contraindications. These include: heart rate less than $60 \mathrm{bpm}$, systolic arterial pressure less than $100 \mathrm{~mm} \mathrm{Hg}$, moderate or severe left ventricular (LV) failure, signs of peripheral hypoperfusion, PR interval greater than 0.24 second, second or third degree AV block, severe chronic obstructive pulmonary disease (COPD), history of asthma, insulin dependent diabetes mellitus (IDDM), and severe peripheral vascular disease (ACC/AHA, 1996). Though patients with relative contraindications are recommended to receive therapy, they need to be monitored for adverse reactions. 


\section{Evidence of Inappropriate Prescribing}

Considerable variation in beta-blocker use for secondary prevention following an AMI has been reported. Rogers et al., examined the National Registry of Myocardial Infarction (NRMI) to investigate beta-blocker use from 1990 to 1993 (Rogers et al., 1994). Oral beta-blockers were used in only $36 \%$ of registered thrombolytic recipients, whereas in patients who did not receive thrombolysis, the utilization was $30 \%$. Soumerai et al., and Krumholz et al., reported results of two recent studies investigating betablocker use. The first study linked Medicare claims data to two databases covering pharmaceutical usage in New Jersey for these Medicare patients. This study reported that while $70 \%$ of the patients were eligible for therapy, only $21 \%$ of these patients received a prescription for beta-blockers (Soumerai, McLaughin, Speilgelman, Hertzmark, Thibault

\& Goldman, 1997). The second study collected data through an intensive chart review of Medicare AMI patients selected from the Cooperative Cardiovascular Project (CCP). The CCP collected data on over 200,000 patients in 45 states over an 8-month period from 1994 to 1995 . This study reported an eligible population of about 39\%, of which only $50 \%$ had a prescription of beta-blocker at discharge (Krumholz, Radford, Wang, Chen, Heiat, \& Marciniak, 1998).

A geographical variation in beta-blocker usage has also been established. There was a significant state and region variation in beta-blocker utilization in the CCP (Krumholz et al., 1998). The 5 states with the highest use were Connecticut (77.1\%), Massachusetts (74.2\%), Maine (68.3\%), New Hampshire (68.9\%), and Vermont (66.7\%). The lowest use occurred in Mississippi (30.2\%), Puerto Rico (32.1\%), Oklahoma (33.5\%), Arkansas (33.5\%), and Nevada (36.4\%). Meehan et al., examined variations in 
a small area using data from six hospitals in Connecticut (Meehan, Hennen, Radford, Petrillo, Elstein, \& Ballard, 1995). The overall utilization of therapy was $41 \%$ among eligible patients. Also, there was a large variation based on hospitals. The utilization varied from $39-54 \%$ for the low-mortality hospitals to $29-33 \%$ for the high mortality hospitals. Pilote et al., also reported regional variation when data from the Global Utilization of Streptokinase and Tissue Plasminogen Activator for Occluded Coronary Arteries trial (GUSTO-1) was analyzed (Pilote et al., 1995). They found that New England had the highest utilization of therapy with $71 \%$ of patients receiving a betablocker prescription at discharge. In comparison, the Mountain and South Central regions had the lowest utilization at $47 \%$ and $49 \%$, respectively. Other studies have reported below optimal beta-blocker utilization for certain patient subgroups such as the elderly, women, and blacks (Fishkind, Paris, \& Aronow, 1997; Pashos, Normand, Garfinkle, Newhouse, Epenstein, \& McNeil, 1994, for details see Chapter 2).

In contrast to the under use in eligible patients, there is also evidence of prescribing of beta-blocker therapy to patients in the presence of contraindications. Brand et al., reported that about $11 \%$ of patients were prescribed therapy in the presence of contraindications (Brand et al., 1995). Another study reported 24\% utilization in patients with potential contraindications (Sial, Malone, Freeman, Battiola, Nachodsky \& Goodwin, 1994).

Thus, despite established guidelines for beta-blocker therapy as secondary prevention following an AMI, there are several reports of instances where eligible patients are not prescribed therapy - thereby establishing under use, and a few reports of patients who are not eligible for therapy being prescribed therapy - thereby establishing 
misuse. Both under use and misuse of therapy are examples of inappropriate use and non-adherence of the 1999 ACC/AHA guidelines.

\section{Consequences of Inappropriate Prescribing}

The inappropriate utilization of any medication can result in adverse patient outcomes. Medication use or prescribing can be considered inappropriate based on a wide variety of criteria such as medication use without indication, medication use with contraindication, over or under dosing, incorrect directions, drug-drug interactions, drugdisease interactions, therapeutic duplication, and longer or shorter duration of therapy than necessary (Schmader, Hanlon, Landsman, Samsa, Lewis \& Weinberger, 1997). For beta-blocker use as a secondary preventive agent in post AMI patients, inappropriate prescribing is usually a result of under use (error of omission) or misuse (error of commission). When patients eligible for beta-blocker use receive a prescription, lower mortality rates have been reported. For example, Krumholz et al., reported a $14 \%$ lower risk of mortality at 1 year after discharge, among eligible patients who received a prescription for beta-blockers at discharge, when compared to eligible patients who did not receive a prescription (Krumholz et al., 1998). In other words, under use was associated with higher mortality. Similarly, Soumerai et al, reported a $43 \%$ lower mortality rate among beta-blocker recipients compared to non-recipients (Soumerai et al., 1997). Additionally, this study also reported that beta-blocker recipients were rehospitalized $22 \%$ less often than non-recipients. Thus, in this study, under use was associated with higher mortality and higher re-infarction rates. Other healthcare 
utilization variables such as physician visits, emergency room visits, length of stay and expenditures have not been investigated so far with regards to under use.

While there is no information on the consequences of misuse of beta-blocker therapy, several studies dealing with other chronic diseases suggest some of the potential consequences of misuse of drug therapy. Shireman et al., demonstrated that drug utilization patterns for asthma, when not consistent with established guidelines, had undesirable outcomes (Shireman, Heaton, Gay, Cluxton \& Moomaw, 2002). Patients receiving excessive doses of beta agonists, an agent that should be used on an as-needed basis, had significantly greater emergency department visits. Anis et al., reported that those asthma patients with inappropriate medication use (defined as excessive use of beta agonists, an as-needed medication, and below optimal use of corticosteroid, a recommended medication), were more likely to be admitted to a hospital, and required more emergency room admissions compared to patients with appropriate medication use (Anis, et al., 2001). This study also reported that those patients with excessive use of beta-agonists visited a significantly greater number of physicians for their prescriptions. A study exploring the monetary effects of inappropriate medication use reported that $77 \%$ of the cost of albumin, a plasma expander, was related to inappropriate use which was defined as use when not necessary, or use when other less expensive therapeutic options were available (Remohi, Arcos, Ramos, Palome, \& Aznar, 2000). An investigation of an increase in prescription drug expenditures associated with peptic ulcer disease in Vermont's Medicaid program was also related to inappropriate use (Soons, Lynch, Seagrave \& Rolley, 1997). Physicians were prescribing H2 antagonists for greater than the recommended 6 to 8 weeks in $60 \%$ of the cases, resulting in a $21 \%$ increase of 
prescription expenditures for gastrointestinal drugs. Finally, a study focusing on 12 major categories of drugs used to treat chronic diseases among older patients revealed that under supply and over supply of medication affected healthcare utilization (Stroupe, Murray, Stump \& Callahn, 2000). Patients with under supply and over supply of medication had greater emergency department visits and hospital admissions compared to patients with a normal supply of medication. Thus, there is evidence that inappropriate medication use can affect patient outcomes. However, there are no studies investigating the outcomes of inappropriate beta-blocker therapy and therefore there is a need to undertake such an investigation.

\section{Physicians' Role in Drug Prescribing}

Many studies have attempted to understand physician's role in beta-blocker therapy. Ayanian et al., examined treatment decisions of cardiologists, internists and family practitioners for their patients with AMI (Ayanian, Hauptman, Guadagnoli, Antman, Pashas \& McNeil, 1994). They found that cardiologists were much more likely to prescribe beta-blockers compared to either internists or family practitioners. Another study compared cardiologists' compliance with practice guidelines for beta-blocker use after an AMI. Of the $60 \%$ of patients, who were eligible for beta-blocker therapy, only $48 \%$ actually received therapy (Brand, et al., 1995). This study also reported that $11 \%$ of patients with contraindications were prescribed beta-blockers. Jollis et al., studied physician specialty and treatment outcomes using the CCP data (Jollis et al., 1996). They found that cardiologists were more aggressive in their treatments and used more 
resources compared to other physicians. Also, their patients had lower mortality rates and better outcomes compared to patients of other physician specialties.

Similar information on other chronic disease treatments reveal that in addition to some of the above characteristics, there are other physician traits that affect prescribing behavior. For example, there is wide variability in preventive aspirin use among teaching and non-teaching hospitals (Venturini, Romero \& Tognoni, 1999). Similarly, younger physicians are more likely to select hypertensive agents consistent with guideline recommendations compared to older physicians (Mehta, Wilcox \& Schulman, 1999). A study that characterized physicians who frequently but inappropriately prescribed longacting benzodiazepines to the elderly, demonstrated that these physicians were more likely to be generalists, have graduated before 1979, practiced in long-term care settings and graduated from medical school from a particular region (Monette, Tamblyn, Mclead \& Gayton, 1997).

In addition to demographic and practice-related factors, knowledge, opinions and beliefs of physicians have also been related to prescribing behavior. For example, a study investigating the reasons for under prescribing of beta-blockers in post AMI patients reported that physicians believed that the adverse effects of beta-blockers would result in a negative quality of life (Kennedy \& Rosensen, 1995). Additionally, they had exaggerated concerns about the relative contraindications, despite reports of benefits to patients with these conditions. This study also reported that commercial influences of pharmaceutical companies contributed to negative attitudes of physicians regarding betablocker therapy. 
There is evidence that despite knowledge of guidelines, certain beliefs of physicians may affect prescribing behavior. For example, Huse et al. explored how well physicians who treat hypertension know the indications and contraindications for particular antihypertensive therapies, and how closely their opinions and practices agreed with national guidelines for their use (Huse, Roht, Alpert \& Hartz, 2001). The study revealed that the degree of specialization and knowledge of indications and contraindications were positively related. However, this knowledge did not result in adherence to treatment guidelines. This was primarily due to study physician's beliefs that the adverse effects of some therapies would affect patient compliance.

Knowledge has also been reported to affect prescribing behavior through interaction with practice characteristics of physicians. For example, a study assessed physician's knowledge, attitudes and prescribing behaviors with regard to the association between Chlamydia pneumoniae and cardiovascular diseases (Sanchez, Jernigan, Strausbaugh, Slemp, Perilla, \& Dowell, 2001). A higher knowledge score was documented among infectious disease specialists and cardiologists than generalists. The study also reported higher knowledge score among those physicians who saw relatively more patients with a myocardial infarction. Additionally, the results revealed that previous experience with managing cardiovascular disease was a good predictor of awareness, knowledge, and the probability of treating patients with anti-microbial agents.

In addition to demographics, practice characteristics, knowledge, attitudes, opinions and beliefs, the interaction of all of these factors can also affect prescribing behavior. Thus, it is important to investigate how these factors individually, and in combination, affect prescribing behavior among physicians for beta-blockers following 
an AMI. Identification of these physician factors could explain the underlying causes of inappropriate prescribing behavior, which would facilitate the targeting of appropriate educational efforts.

\section{STATEMENT OF PROBLEM}

Beta-blockers have been demonstrated to yield better health outcomes when used for secondary prevention in post AMI patients. However, in many instances it is inappropriately prescribed in such patients. There is evidence of under-prescribing in patients who should be prescribed, and there is some, although limited, evidence of prescribing in patients with contraindications. Many studies have investigated the effect of under prescribing of therapy on mortality and morbidity. However, these have examined data from clinical trials, which could be different from real life use or have investigated the elderly population in Medicare, which is a unique population segment. There has been very little literature examining the effects of this under prescribing on healthcare utilization and expenditures. Additionally, literature that examines patient outcomes due to misuse of therapy is lacking. Thus, there is a need to determine the effect of inappropriate prescribing on patient outcomes.

In addition to examining the effects of inappropriate prescribing on patient outcomes, this study will also explore the association of physicians' knowledge and willingness to prescribe beta-blocker therapy with their prescribing behavior. Previous studies have found ample variation of medication use by physician characteristics (demographic, practice and knowledge). However, there is a need to understand how these factors interact individually and in combination with prescribing behavior for beta- 
blockers post AMI. An understanding of these physician factors would facilitate in developing and targeting educational efforts towards changing prescribing behavior to be consistent with the published guidelines.

\section{CONCEPTUAL FRAMEWORK}

In Phase I, the study will evaluate the impact of appropriate and inappropriate prescribing of beta-blocker therapy by physicians, following an AMI, on patient outcomes and utilization of health services. In Phase II, the study will determine physician-related factors that are related to beta-blocker prescribing in post-AMI patients. Phase I will involve the use of secondary data and Phase II will require primary data collection (Figure 1).

\section{Phase I}

The following sections will describe the rational for selection of the Medicaid population for this study, cohort definition, classification of patients, risk adjustment and evaluation of patient outcomes that will be required for this phase of the study.

\section{$\underline{\text { Selection of Medicaid Population }}$}

This study will examine and compare the effects of appropriate and inappropriate prescribing of beta-blockers therapy as a post AMI secondary preventive agent on patient outcomes in a Medicaid population. Medicaid programs provide health insurance coverage to its members for physician visits, inpatient and outpatient hospitalizations, 


\section{Figure 1: Conceptual Framework}

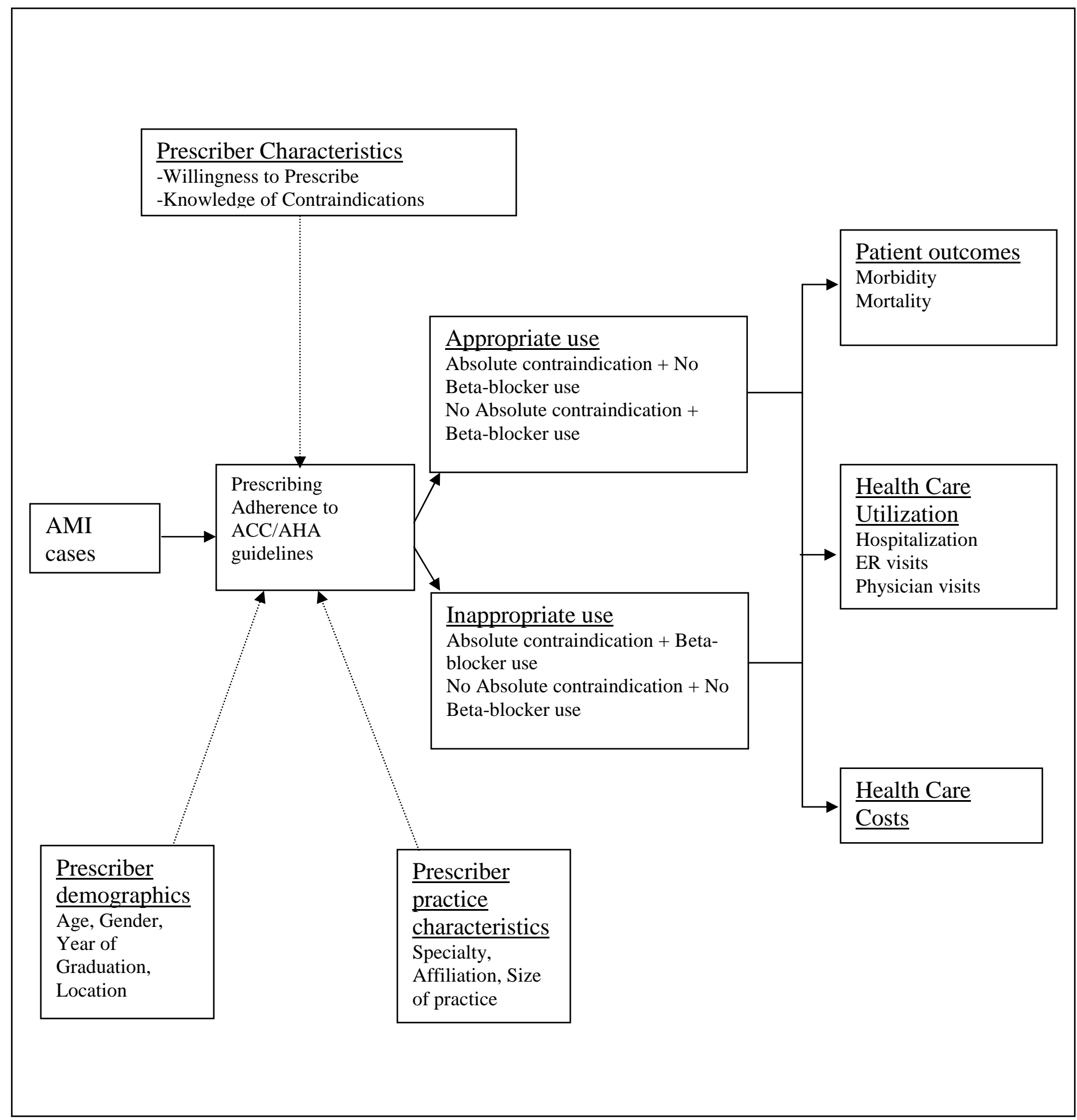

AMI: Acute myocardial infarction, ACC: American College of Cardiology, AHA: American Heart Association, ER: Emergency room 
laboratory tests, nursing home care, family planning services and supplies, home health care and pharmaceuticals. Thus, a retrospective analysis of Medicaid claims data can provide all necessary information about study patients in one place. Also, the effect of post AMI beta-blocker use has not been previously investigated in this population. While AMI has been established as a condition prevalent in the elderly, there is substantial evidence that it is common among younger people, too. Consequently the age of the patient population (less than 65 years of age) is not as big a disadvantage. In support, a recent study at the University of Michigan revealed that more than 1 in 10 patient with AMI is less than 46 years old. (Doughty, et al., 2002). Specifically, data from the West Virginia (WV) Medicaid population will be utilized for Phase I of this study.

\section{Cohort Definition}

The WV Medicaid program maintains data on each medical and pharmaceutical paid claim for its recipients from providers for health care services. The data includes the date when the service was provided, type of service, amount paid, type of provider, and recipient number. Information about utilization and expenditures for services for individual recipients can be retrieved for various time periods from the claims data. For this study, paid claims data from January 1, 1996 to June 30, 2001 will be used. All AMI patients who are less than 64 years of age and are not in managed care during the years 1997, 1998, 1999, and up to June 30, 2000 will be extracted. Recipients who are 65 years or older will not be included to avoid the issue of incomplete information since they will have Medicare as their primary payer for health services. For similar reasons, patients who are part of managed care will also be excluded. 
For this cohort of AMI patients, those patients whose information is missing after being initially present will be used to investigate the effect of appropriate or inappropriate beta-blocker prescribing on mortality. Patients who have follow-up information and are continuously eligible for a period of 12 months after the incidence of AMI will be considered to study the effect of appropriate or inappropriate beta-blocker prescribing on morbidity and health care utilization. The age-limit is set at 64 to avoid including those patients who will become eligible for Medicare, during the period of follow-up after being initially present. Claims data for services, prescriptions, and hospitalizations for a period of one year before the incident AMI will be extracted for each patient. This information will be used for risk adjustment of the patient, and to determine eligibility of the patient to receive beta-blockers after an AMI. Therefore, for each patient being investigated for morbidity and health care utilization, there will be one year of data prior to the incident AMI and one year of data following the incident AMI (Figure 2).

\section{Classification of Patients}

The AHA/ACC guidelines divide patients into three classes with respect to any therapy and/or procedure - Class I, Class II and Class III (ACC/AHA, 1996). Class I includes those patients with conditions for which there is evidence and/or general agreement that the therapy is beneficial, useful, and effective. Class II is sub-divided into IIa and IIb. Class IIa includes patients with conditions for which the weight of evidence/opinion is in favor of usefulness/efficacy of therapy and Class IIb includes patients with conditions for which usefulness/efficacy of therapy is less well established by evidence/opinion. Class III includes patients with conditions in the presence of which 


\section{Figure 2: Time Line for Phase I}

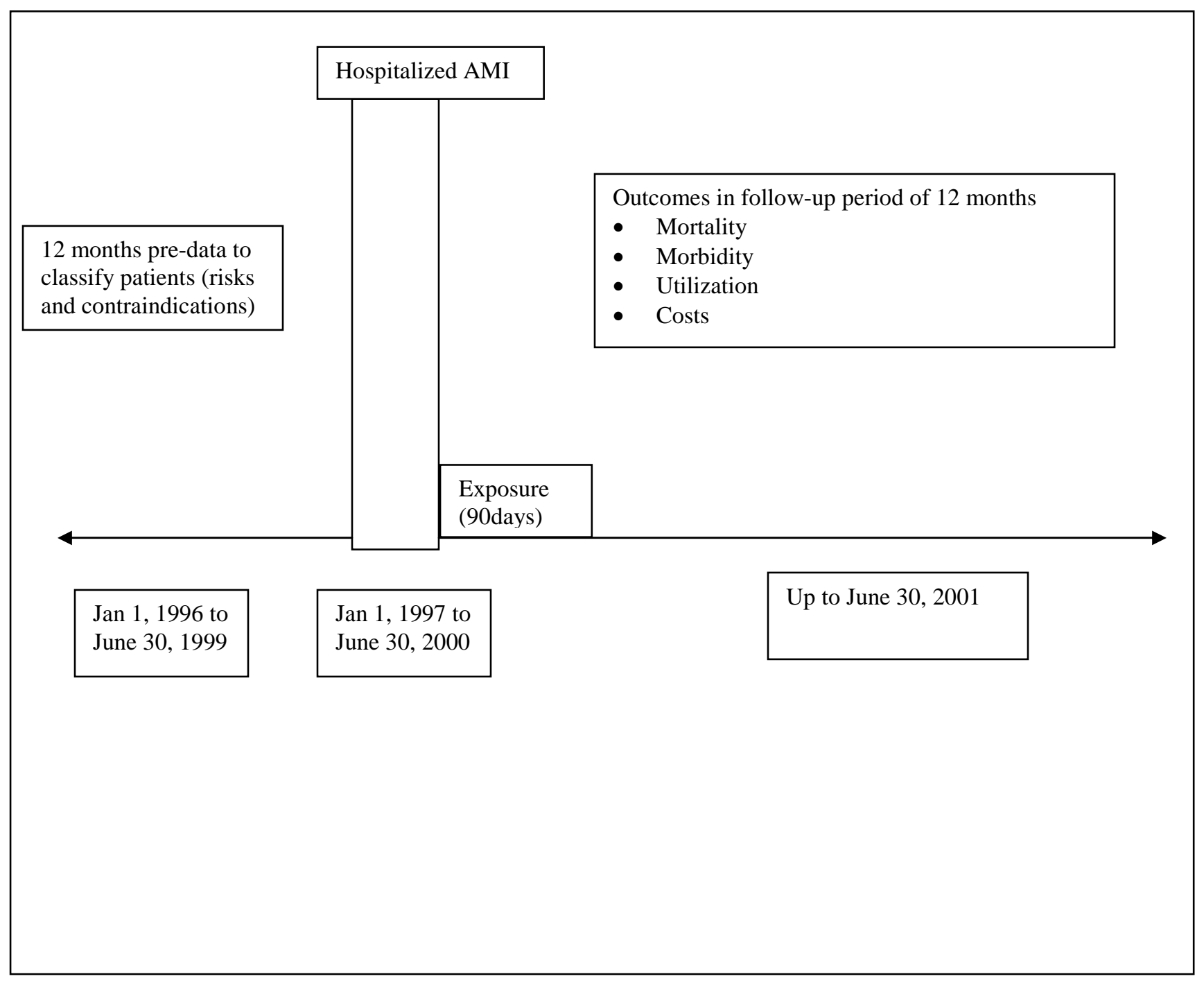

AMI: Acute myocardial infarction 
there is evidence and/or general agreement that a therapy is not useful/effective, and in some cases may be harmful. For beta-blocker therapy, Class I patients are non low-risk without a clear contraindication to beta-blocker therapy and survivors of non-ST-segment elevation (ACC/AHA, 1999). Class IIa includes low-risk patients without a clear contraindication to beta-blocker therapy and survivors of non-ST-segment elevation AMI. Class IIb are patients with moderate or severe left ventricular failure. Patients with relative contraindications to beta-blocker therapy are also included in this class, provided they are monitored closely. None of these patients belong to Class III. Apart from the above classifications, these guidelines also state that high-risk patients would be those with evidence of large or anterior infarction. Additionally, low risk patients include those without: previous infarction, anterior infarction, advanced age, complex ventricular ectopy, or hemodynamic evidence of LV systolic dysfunction.

Based on the above guidelines and recent reports, for all practical purposes patients can be classified as Class I, Class II and Class III for receiving beta-blocker prescription for secondary prevention following an AMI (ACC/AHA, 1999; Philips et al., 2000). A Class I and Class II patient for beta-blocker therapy at hospital discharge is one who has no absolute contraindications to therapy. Patients with relative contraindications are also included in this category (Class II). A patient would be Class III for beta-blocker therapy at hospital discharge if the patient has absolute contraindications to beta-blocker therapy. For study purposes, patient data during the year before the incident AMI and at the time of the incident AMI will be utilized from claims for this classification.

After classifying the patients into the afore-mentioned three categories, the prescription of beta-blockers during discharge can be determined from the prescription 
claims data. Based on appropriate and inappropriate prescribing criteria described above, the AMI patients in the study can be further divided into two groups - the appropriately prescribed and the inappropriately prescribed. Patients who are classified as Class I and Class II and receive therapy and patients who are classified as Class III and do not receive therapy will be categorized as "appropriately prescribed". In contrast to this, patients who are classified as Class I and Class II and do not receive therapy and patients who are classified as Class III but receive therapy will be categorized as "inappropriately prescribed".

\section{$\underline{\text { Risk Adjustment }}$}

In observational studies, investigators have no control over inherent risks that patients bring into the study. Therefore, large differences on observed covariates in the selected groups may exist, and these differences can lead to biased estimates of outcomes. An important statistical technique developed to overcome this kind of selection bias is estimation of propensity scores. This method involves estimating the conditional probability of an event such as receiving specific therapy, on a treatment outcome, based on observable characteristics. The propensity score calculated from observational characteristics of patients are then used to reduce bias and increase precision. The three commonly used techniques that use propensity scores are matching, stratification (also called sub-classification), and regression adjustment (D'Agostinio, 1998). Each of these techniques is a way of making an adjustment for covariates prior to (matching and stratification) or during (stratification and regression adjustment) 
calculation of the treatment effect. While the method of calculation of the propensity score is the same in all three techniques, the application is different.

In matching, control subject selection is based on whether they match with the treated subjects on background covariates that the investigator believes need to be controlled (D’Agostinio, 1998). However, it is often difficult to find subjects similar in all important covariates even when there are only a few background covariates of interest. In stratification, the subjects are grouped into strata determined by the observed background characteristics (D'Agostinio, 1998). Once strata are defined, treated and control subjects in the same stratum are compared directly. The number of strata depends on the number of covariates. For example, if all the covariates are dichotomous then there would be $2^{\mathrm{k}}$ subclasses for $\mathrm{k}$ covariates. If $\mathrm{k}$ is large, then some strata might contain subjects from only the treated group, which would make it impossible to estimate a treatment effect in that stratum. In regression adjustment, a large set of background covariates are used to estimate the propensity score, which is then used to adjust the final estimate of the treatment effect (D’Agostinio, 1998). Here the investigator can include only a subset of the most important variables and the propensity score in the final model.

The proposed study involves two groups of patients, those that were appropriately prescribed beta-blocker therapy and those that were inappropriately prescribed betablocker therapy. Irrespective of whether the patients receive therapy appropriately or not, there are certain patient characteristics that affect prognosis and outcomes after an AMI. For example, a younger patient (age <50) is likely to have a better recovery from an AMI when compared to an older patient. This would be true regardless of whether the patient received beta-blocker therapy appropriately or not. Similarly, a patient who has had a 
previous AMI would have a worse prognosis, and thus would have more adverse outcomes after an AMI. There are a variety of such patient conditions and characteristics that affect patient outcomes after an AMI but are not related to beta-blocker prescribing. It is possible that patients in the two groups, appropriately prescribed and inappropriately prescribed, differ based upon these characteristics. These characteristics require to be adjusted for patients before the effect of appropriate and inappropriate prescribing on outcomes can be determined. Therefore, selection bias due to the inherent risk of patients in this study needs to be accounted for to get a more precise effect of appropriate/inappropriate prescribing of beta-blocker therapy on patient outcomes. A propensity score that combines all background covariates will be utilized to adjust for differences in the study groups. This propensity score can then be utilized in all the analysis where the effect of appropriate/inappropriate prescribing on patient outcomes will be assessed.

The different characteristics which will be utilized in estimating this propensity score are demographic characteristics such as age, gender, and/or race; presence of relative contraindications for beta-blocker therapy; medical history such as presence of diabetes, hypertension, previous AMI, previous congestive heart failure, previous angioplasty or open heart surgery, stroke; procedures during the incident AMI such as percutaneous coronary transluminal angioplasty (PCTA), and coronary artery bypass graft (CABG); length of stay during incident AMI; other discharge medications such as aspirin, Angiotensin converting enzyme (ACE) inhibitors, calcium channel blockers, and loop diuretics; and use of beta-blockers before the incident AMI. These characteristics have been demonstrated to affect outcomes for AMI patients (Soumerai et al., 1997). 
Due to the large number of characteristics and conditions that need to be adjusted, regression adjustment would be the best method of applying the estimated propensity score.

\section{$\underline{\text { Evaluation of Outcomes }}$}

Beta-blocker therapy when appropriately used for secondary prevention following an AMI has been demonstrated to reduce cardiovascular morbidity and mortality. Thus, the selected cohort of appropriately prescribed and inappropriately prescribed patients will be followed to detect changes in cardiovascular mortality and morbidity related to the use of beta-blocker therapy. Beta-blockers are associated with maximum reduction of risk for cardiovascular death and morbidity in the first few years after an AMI (Philips et al., 2000, Goldman et al., 1988). The protective effect provided by beta-blockers has been reported to subside in the subsequent years. In this study, the patients will be followed for a period of one year after the incidence of AMI. Another reason for restricting the follow-up period to 1 year is that the number of continuously eligible patients decreases as the number of years increase. Thus, when multiple years are involved, the number of patients in the cohort would be small and pose problems of power for statistical tests.

Medicaid data contains information on the diagnostic and therapeutic procedures performed on all continuously eligible patients who have utilized health care services. Patients, who stop having information after being present in the system initially, do so for several reasons. They may have become ineligible to receive Medicaid benefits, have moved away, or died (which can be confirmed with death certificate information 
available from the WV Bureau of Vital Statistics and the National Death Index (NDI)). However, the unique ID of those patients with missing information during the follow-up period can be used to determine the actual reason for the missing information. If the person with the missing information is confirmed to be dead from Vital Statistics records, the cause of death can be obtained from their death certificates. If the cause is related to cardiovascular illness such as an AMI, heart failure, stroke, angina, other forms of ischemia, essential hypertension, coronary artery disease/artherosclerosis or cardiogenic shock, these patients will be involved in investigating the effect of appropriate/inappropriate prescribing of beta-blockers on mortality using survival analysis. An increase in mortality in the inappropriately prescribed group will be proof of poor outcomes in this group.

Among patients with follow-up information, those eligible in the 12-month period after the incident AMI will be examined for morbidity and the extent of health care utilization. Claims associated with cardiac conditions and procedures will be extracted to study outcomes in the follow-up period. An increase in cardiac conditions for the inappropriately prescribed group compared to the appropriately prescribed group will provide evidence of poor outcomes associated with morbidity for this group. The various patient characteristics that affect treatment outcomes will be adjusted for by using them to estimate a propensity score. This score along with group status (inappropriate or appropriate) can then be included in a survival analysis where the dependent variable will be the time to first hospitalization due to an event. Here the event will be any cardiovascular event such as a successive AMI, heart failure, stroke, angina, and other forms of ischemia, essential hypertension, cardiogenic shock, disease of endocardium, 
conduction disorders, or cardiomyopathy. In addition to the incidence of these conditions in the follow-up period, survival analysis will be used to evaluate the distribution of the time to incidence of these events. Studying the distribution of incidence will give a better idea about the effect of appropriate and inappropriate beta-blocker prescribing on outcomes. This is preferable to assessing differences in patients outcomes based on number of incidence of morbidity data alone.

Similarly, to study the effect on healthcare service utilization for the above mentioned cardiac conditions, the number of physician visits, hospitalization episodes, length of stay, and emergency room visits will be compared between patients in the appropriately prescribed and inappropriately prescribed cohorts. The hypothesis is that there is no difference in utilization of these services between the inappropriately prescribed group and the appropriately prescribed group. Regression analysis will be conducted with each of these utilization-related variables with group status as the independent variable. Patient characteristics that affect treatment outcomes will be adjusted for by using the propensity score as a co-variate. Regression analysis is a better way to compare these utilization-related variables as it allows for adjustment of multiple patient factors compared to other methods such as analysis of variance (ANOVA).

\section{Phase II}

The purpose of phase II of the study is to determine physician-related factors that are associated with prescribing of beta-blockers for secondary prevention of cardiac problems after an AMI. This phase of the study will involve surveying physicians in the specialties of cardiology, internal medicine, family practice and general practice in the state of WV (Figure 3). 


\section{Figure 3: Phase II}

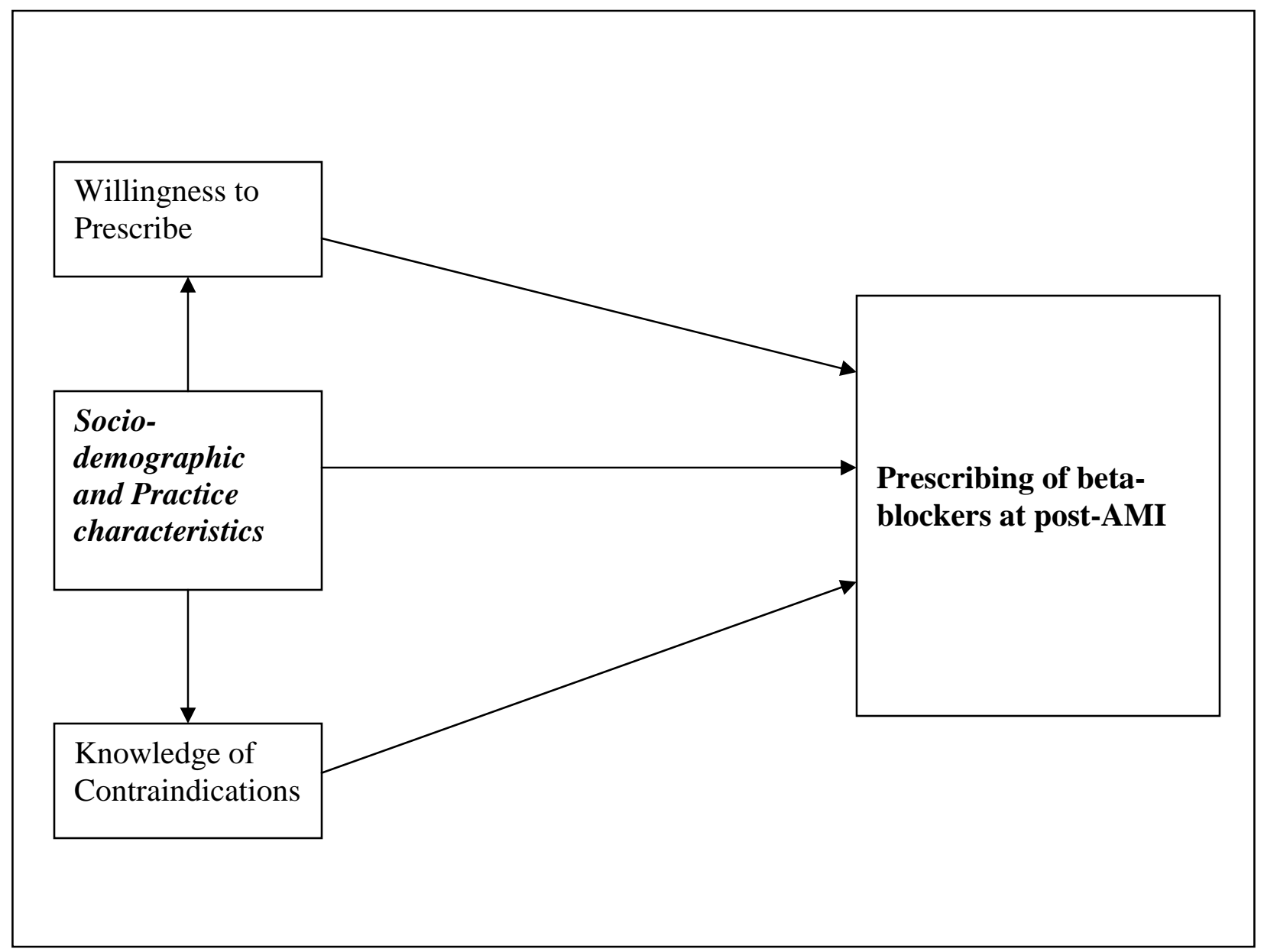

AMI- Acute myocardial infarction 
The target physicians will be sent a survey that measures the following: knowledge about contraindications to beta-blocker therapy, willingness to prescribe betablockers, practice characteristics and socio-demographics. One major reason for the nonprescribing of beta-blockers in patients without contraindications is that internists and family practitioners have been shown to be less aware or less certain than cardiologists of the cardiovascular medical advances established through clinical trails (Bradford et al, 1999). Thus, the survey will be designed to assess physicians' knowledge about contraindications for beta-blocker therapy post-AMI, to determine whether or not there are differences in knowledge when compared by specialty. Specifically, they will be compared for (1) knowledge of absolute contraindications when therapy with betablocker should not be initiated; and (2) knowledge of relative contraindications when therapy with beta-blockers can be, and should be, initiated.

In addition to knowledge, physician willingness to prescribe beta-blocker therapy for post AMI patients will be explored. A section will be dedicated to inquire about physicians' willingness to prescribe beta-blockers as post-AMI therapy in patients with different ailments.

The last section of the survey will focus on socio-demographic and practice characteristics. These have been reported to affect the provider's knowledge, beliefs, and practice (Ayanian et al., 1994). Specific characteristics reported include age, gender, specialty of practice, year of board certification in the primary field of practice, number of patients treated within a specific time, number of beds at practice site, and whether practice site is associated with teaching status. 
Physician prescribing behavior for beta-blockers will be determined by asking the respondents to recall the number of AMI patients that they prescribed long-term betablockers for secondary prevention, out of the last 10 AMI patients treated. Percent prescribing rate will be calculated based on the response of the physicians [i.e. (response/10)*100]. This will be used as a proxy for prescribing behavior. Though this is not the best method of measuring prescribing behavior, questions which ask the respondents the average number of post-AMI patients prescribed beta-blocker in the past month or longer duration of time will involve higher recall bias and thus may be unreliable. Also, whether the prescribing of the physicians for the last 10 patients was appropriate or inappropriate cannot be determined through a survey. This is because prescribing is unique for every patient and depends on patient co-morbidties. To obtain detailed information for each individual patient would involve a very lengthy survey, which can increase burden for the respondents for time of completion and effort to recall each specific case. This can also result in a very low response rate. Thus, given the time and resource constraints this method of getting a proxy for prescribing behavior appears to be the best.

The association of this estimated prescribing rate or the proxy prescribing behavior will be statistically investigated with knowledge of contraindications, willingness to prescribe and physicians socio-demographic and practice characteristics, individually and in combination. A sub-analysis will also be conducted to determine the association of socio-demographic and practice characteristics on physicians' knowledge of contraindications and willingness to prescribe. Thus, this phase of the study will give 
a better understanding of the role of physician characteristics that affect their prescribing behavior.

\section{STUDY GOALS, AND RESEARCH QUESTIONS}

\section{Phase I}

The overall goal of phase I is to study prescribing patterns of beta-blocker therapy in Medicaid patients following an AMI and its effect on patient outcomes. Specific research questions necessary to achieve this goal are as follows:

\section{Research Question 1:}

To what extent do physicians adhere to AHA/ACC guidelines for appropriate betablocker therapy for secondary prevention in patients who have had an AMI in the WV Medicaid program?

Rationale: This is an exploratory question to assess the prescribing of beta-blockers in the WV Medicaid population with regards to published guidelines and to classify them as appropriate or inappropriate. Medicaid patients have not been studied before for such an investigation. Thus, it would determine whether or not prescribing of beta-blockers in post-AMI Medicaid patients is similar to those reported in other patient populations such as the elderly (Medicare), and those in managed care.

\section{Research Question 2:}

What is the relationship between appropriate and inappropriate prescribing of betablockers following an AMI and all cause and cardiac mortality in WV Medicaid? 
Null Hypothesis A: There is no difference in all cause mortality rates between AMI patients who receive appropriate beta-blocker therapy and those who receive inappropriate beta-blocker therapy for secondary prevention.

Null Hypothesis B: There is no difference in cardiac mortality rates between AMI patients who receive appropriate beta-blocker therapy and those who receive inappropriate betablocker therapy for secondary prevention.

\section{Research Question 3:}

What is the relationship between appropriate and inappropriate prescribing of betablockers following an AMI and subsequent morbidity due to the following conditions in the WV Medicaid program (irrespective of whether they suffered from these conditions prior to the AMI under consideration)?

- Angina

- Cardiogenic shock

- Disease of endocardium, conduction disorders \& cardiomyopathy

- Essential hypertension

- Heart failure

- Other ischemic disease

- Stroke

- Successive or recurrent AMI

Null Hypothesis $C$ : There is no difference in cardiovascular morbidity between AMI patients who receive appropriate beta-blocker therapy and those who receive inappropriate beta-blocker therapy for secondary prevention. 


\section{Research Question 4:}

What is the relationship between appropriate and inappropriate prescribing of betablockers following an AMI and utilization of health care services (physician services, hospitalization services, hospital length of stay, ER visits) and expenditures due to the following conditions in WV Medicaid (irrespective of whether they suffered from these conditions prior to the AMI under consideration)?

- Angina

- Cardiogenic shock

- Disease of endocardium, conduction disorders \& cardiomyopathy

- Essential hypertension

- Heart failure

- Other ischemic disease

- Stroke

- Successive or recurrent AMI

Null Hypothesis D: There is no difference in utilization of services due to the above listed conditions between AMI patients who receive appropriate beta-blocker therapy and those who receive inappropriate beta-blocker therapy for secondary prevention.

Null Hypothesis E: There is no difference in costs due to the above listed conditions between AMI patients who receive appropriate beta-blocker therapy and those who receive inappropriate beta-blocker therapy for secondary prevention.

\section{Phase II}

The goal of phase II is to determine how physician characteristics such as knowledge, willingness to prescribe, demographic and practice characteristics, and interactions of these different characteristics affect their prescribing behavior for beta- 
blockers as secondary preventive agents after an AMI. Specific research questions for this phase are as follows:

\section{Research Question 5:}

How does WV physicians' knowledge of absolute and relative contraindications of betablocker therapy affect their self-reported prescribing behavior of this therapy as a secondary preventive agent following an AMI?

Null Hypothesis F: There is no relationship between knowledge of absolute and relative contraindications of beta-blocker therapy and physician prescribing behavior of this therapy as a secondary preventive agent.

\section{Research Question 6:}

How does WV physicians' willingness to prescribe beta-blocker therapy affect their selfreported prescribing behavior of this therapy as a secondary preventive agent following an AMI?

Null Hypothesis G: There is no relationship between WV physicians' willingness to prescribe beta-blocker therapy and their prescribing behavior of this therapy as a secondary preventive agent following an AMI.

\section{Research Question 7:}

How does demographic (age, gender, location, year of graduation) and practice (specialty, affiliation, size of primary practice hospital) characteristics of WV physicians 
affect their self-reported prescribing behavior of beta-blocker therapy as a secondary preventive agent following an AMI?

Null Hypothesis $H$ : There is no relationship between demographic and practice characteristics of WV physicians and their prescribing behavior of beta-blocker therapy as a secondary preventive agent following an AMI.

\section{Research Question 8:}

How does demographic (age, gender, location, year of graduation) and practice (specialty, affiliation, size of primary practice hospital,) characteristics of WV physicians affect their knowledge and willingness to prescribe beta-blocker therapy as a secondary preventive agent following an AMI?

Null Hypothesis I: There is no relationship between demographic and practice characteristics of WV physicians and their knowledge of absolute and relative contraindications of beta-blocker therapy as a secondary preventive agent following an AMI.

Null Hypothesis J: There is no relationship between demographic and practice characteristics of WV physicians and their willingness to prescribe beta-blocker therapy as a secondary preventive agent following an AMI.

\section{Research Question 9:}

Which factors among physician demographic and practice characteristics, physicians' knowledge of beta-blocker contraindications, and willingness to prescribe them, are the best predictors of their self-reported prescribing behavior of beta-blocker therapy as a 
secondary preventive agent following an AMI? (This simultaneously controls for all aspects described in research question $5,6 \& 7$ ).

Null Hypothesis K: There is no association between WV physicians' prescribing of betablocker therapy as a secondary preventive agent following an AMI and physician factors (demographic and practice characteristics, knowledge of beta-blocker contraindications, and willingness to prescribe).

\section{Research Question 10:}

What are the reasons reported by physicians for not prescribing beta-blockers in postAMI patients?

Rationale: A previous study reported reasons such as adverse effects, exaggerated concerns for relative contraindications and commercial influences of pharmaceutical companies as reasons for under prescribing of beta-blockers as post-AMI medications (Kennedy \& Rosensen, 1995). This research question is a follow-up on this report to determine whether the reasons for not prescribing beta-blockers in post-AMI patients are the same or have changed over the years.

\section{STUDY SIGNIFICANCE}

In Phase I, the effect of appropriate/inappropriate prescribing of beta-blockers by physicians on patient outcomes following an AMI will be evaluated in this study. In Phase II, physician characteristics that affect prescribing behavior of beta-blocker therapy post AMI will be determined. Information from Phase I will help in identifying consequences of inappropriate prescribing if any, such as mortality, morbidity, health 
care utilization (hospitalization, ER visits, and physician visits) and health care expenditures from a payer's perspective. Based on past studies, it is hypothesized that inappropriate use may increase mortality and morbidity in those patients and consequently increase health care utilization and expenditures. This is vital information from a payer's perspective because it provides information about both the quality of care provided to its members as well as cost-efficiencies within the financing of the system. Beta-blockers are a very inexpensive way of ensuring the quality of care by reducing mortality and morbidity received by post-AMI patients, and concurrently reducing costs due to unnecessary health care services utilization. This is a good example in which improving the quality of care and reducing costs are not competing interests. The above concerns are especially important from the perspective of a Medicaid program, which is a public health insurance program and supported by federal and state tax dollars.

Phase II will help in understanding physician related factors that are associated with prescribing of beta-blocker therapy following an AMI. This information can be utilized in designing active interventions to meet specific needs such as group or one-onone education, and individual outreach visits. Implementation of these active interventions will have better results in comparison to passive interventions such as dissemination of printed materials, reminders at the time of prescribing, and formulary control, which do not involve physician input (Figueiras, Sastre \& Gestal-Otero, 2001). Therefore, these active interventions may be more successful in improving prescribing behavior of physicians for secondary prevention following an AMI with beta-blockers, and more likely to lead to better adherence of guidelines. 
Identifying the consequences of inappropriate beta-blocker use and the factors contributing to physicians prescribing of beta-blockers are the first steps towards improving quality of care provided to AMI patients. While quality concerns by itself is a strong reason, reducing the avoidable health care utilization and expenditures is also vital to a system that is facing ever-increasing costs.

\section{STUDY LIMITATIONS}

The limitations of each phase of the study are discussed below:

In Phase I, the limitations are associated with the data being used. Medicaid data does not include utilization claims for individuals aged 65 years and over and these patients will not be a part of the study cohort. Patients who are a part of managed care will also be excluded due to lack of utilization claims data. Furthermore, patients who do not have continuous eligibility during the study period will be excluded. These selection criteria will exclude a significant portion of the Medicaid recipients. Thus, whether the effect of inappropriate prescribing is different for the excluded group cannot be determined and is beyond the scope of this study. Also, AMI is a condition more predominant in the elderly. Thus, when a younger group such as Medicaid patients is used to study this condition, the number of patients in the study will be relatively lower when compared to previous studies that involved the elderly. This may pose problems with power and effect size when complex statistical analyses are involved. Also, younger patients with AMI will have better prognosis than older patients, thus results from this study may not be extended to older patients. 
Another limitation is the fact that demographic characteristics of people from West Virginia may not necessarily be similar to demographic characteristics of other states. Therefore, results may not be generalized to other states. The study will use claims data and this does not allow adjusting for all the contraindications for betablockers, as some of them do not have an ICD-9 (International classification of disease, $9^{\text {th }}$ edition) code. For example, peripheral hypoperfusion, intolerance or allergies to betablockers are relative contraindications which cannot be identified. However, ICD-9 code for peripheral vascular disease can be used as a proxy for patients with peripheral hypoperfusion and the percentage of patients allergic to beta-blockers has been reported to be low ranging from $7.7 \%$ to $12.5 \%$ in previous studies (Phillips et al., 2000). Some of the attributes which classify patient risk levels can be identified only from patient charts and are not coded in patient claims. Therefore, true risk levels of the patients cannot be identified through claims data and thus the study will be unable to adjust for severity of the AMI. Since claims data is being used to determine presence and absence of conditions, errors due to billing and coding cannot be ruled out. Also, the study will not be able to consider the effect of other possible confounding factors such as obesity, smoking, or other life-style related conditions, which are not included in claims data.

Some of the limitations of Phase II are inherent due to the cross-sectional study design method of data collection i.e. mail survey. Factors that affect prescribing behavior such as disease severity, multiple co-morbidities, and patient choice will not ascertained through the survey used, and thus cannot be included in the analysis. A major limitation is that prescribing percent, which is the proxy for prescribing behavior, will be computed based on last 10 patients treated and may not be representative of the physicians 
prescribing behavior in general. Also, prescribing percent calculated will not reflect whether prescribing was appropriate or inappropriate. Thus, in reality association of physician related factors with prescribing percent will be investigated and not their association with appropriate and inappropriate prescribing percent.

Mail surveys usually have a response rate between 30-40 percent (Dillman, 1978). Hence, generalization of the results obtained to those who did not respond may be limited. There exists a lack of control over item non-response in mail surveys. Mail surveys are insensitive to substitution of respondents, thus whether the physician answered the survey or someone else cannot be determined. The questions in the survey can be interpreted differently by different respondents and thus it can fail to provide standardized understanding of survey questions among respondents. Other limitations such as recall bias, and limitations to recall, may also exist for the section associated with knowledge and prescribing behavior.

This chapter gave a brief introduction to the problem, the need, the research questions, and the significance of the study. The next chapter will give a extensive review of the existing literature associated with AMI, and beta-blocker therapy. 


\section{CHAPTER TWO \\ REVIEW OF LITERATURE}

This literature review will provide a background to understand - acute myocardial infarction (AMI), beta-blocker drug therapy, their effectiveness, recommendations for their use, variation in their use, implications of this variation in use and the physicians role in this variation of use. The final section in the chapter will provide a brief description of the Medicaid program and West Virginia (WV) Medicaid, the sample frame for this study.

\section{Acute Myocardial Infarction}

Acute Myocardial Infarction (AMI) is the death of heart muscle due to the loss of blood supply (Bellenir, 2000). This loss of blood supply is usually caused by a complete blockage of a coronary artery by a blood clot. Coronary arteries are blood vessels, which supply blood to the heart muscle. Coronary artery disease (CAD) is the primary underlying process that leads to AMI (Stringer \& Lopez, 1999). Cholesterol, a fatty chemical, is a part of the outer lining of cells in the body. Cholesterol plaque is the formation of a hard, thick substance on the artery walls, which is caused by the deposition of cholesterol on the artery walls, a process that begins early in life. With time, this accumulation causes thickening of the artery walls and narrowing of the arteries, a process called as atherosclerosis. Plaque accumulation can be accelerated by specific risk factors such as hypertension, diabetes mellitus, smoking, and hyperlipidemia. Atherosclerosis can progress and cause significant narrowing of the 
coronary arteries. These narrowed arteries cannot increase the blood supply to meet the increase in oxygen demand by the heart muscle during exercise or excitement. This condition called ischemia gives rise to chest pain or angina. However, this is not the etiology of AMI. The surface of a cholesterol plaque can become sticky due to a rupture or fissure, precipitating thrombus formation. When a blood clot forms on top of this plaque, the artery becomes completely blocked, causing the death of the heart muscle or AMI.

Pain or pressure in the chest is a common symptom of AMI (Bellenir, 2000). AMI's most frequently occur from 4 AM to $10 \mathrm{AM}$ due to high adrenaline amounts released from the adrenal glands during the morning hours. AMI's do not usually occur during exercise, although exercise is commonly associated with angina. About onefourth of AMI's are silent, without chest pain. In addition to chest pain, patients may complain of sweating, jaw pain, heartburn, arm pain, indigestion, back pain, general malaise, nausea and shortness of breath.

\section{$\underline{\text { Diagnosis }}$}

Initial diagnosis is made by a combination of clinical symptoms and electrocardiogram (EKG) changes (Bellenir, 2000). An EKG is a recording of the electrical activity of the heart and can detect areas of muscle deprived of oxygen and /or dead tissue in the heart. However, confirmation of an AMI can only be made hours later through detection of elevated creatinine phosphokinase (CPK) in the blood. CPK is muscle enzyme released into the blood by dying heart muscles when their surrounding membranes dissolve. 


\section{$\underline{\text { Treatment }}$}

The primary goal of the treatment is to open the blocked arteries and restore blood flow to the heart muscles, called as reperfusion (Bellenir, 2000; Stringer \& Lopez, 1999). Once the artery is open, the patient becomes pain free. Early reperfusion minimizes the extent of damage to the heart muscle and preserves its pumping function. Delay in reperfusion can result in irreversible death to the heart muscle cells and may reduce the pumping force of the remaining heart muscle. The future quality of life and longevity for the patients depends on the amount and health of the remaining heart muscle.

The fastest method of opening a blocked artery is to perform a percutaneous transluminal coronary angioplasty (PTCA). Here a tiny plastic catheter with a balloon at the end is advanced over a fine guide wire to the site of blockage and inflated, under Xray guidance. This pushes the plaque and clot out of the way. PTCA can be effective in opening up to $95 \%$ arteries within 60 minutes. Medications if given early are also effective in opening arteries. Clot dissolving medications also called as thrombolytic agents such as tissue plasminogen activator (t-PA) and Streptokinase are given intravenously. These are reported to open $80 \%$ of the blocked arteries within 90 minutes.

Anti-platelet agents, such as aspirin, reduce platelet clumping and clot-formation. This decreases the recurrent closure of artery and improves the chances of survival. Anticoagulant agents, such as heparin, given intravenously, act as a blood-thinning agent and prevent blood clots and maintains the artery open during the initial 24 hours. Nitroglycerin, a vasodilator, is given either under the tongue or intravenously, to prevent blood vessel spasm and minimize the area of the heart attack. Angiotensin-converting enzyme (ACE) inhibitors are given orally after an AMI to improve the heart muscle 
healing process. Beta-blockers act as antiarrhythmic and are given either intravenously or orally to decrease the magnitude of infarction and incidence of associated complications and the rate of infarction in patients (Bellenir, 2000). Other antiarrhythmics such as lidocaine, amiodarone, procainamide can also be used. Other classes of drugs such as calcium channel blockers, e.g. nifedine, verampil and inotropic agents such as digitalis may also be used [American College of Cardiology (ACC)/American Heart Association (AHA), 1996]. When PTCA and medications fail to achieve reperfusion or maintain open arteries, patients are subjected to coronary artery bypass graft (CABG) surgery (Bellenir, 2000).

Patients are monitored in the hospital for 3-6 days prior to discharge. Rhythm disturbances, shortness of breath, or recurrent pain are indications for further therapy either through PTCA, medications, or bypass surgery (Bellenir, 2000). Patients gradually increase their activity under observation. Before discharge, stress tests are useful for detecting any narrowing in the coronary arteries, rhythm changes, and heart muscle failure. These also help in prescribing discharge medications. Several weeks are needed for the heart to heal before resuming full activity. After a small AMI, patients can resume normal activities after 2 weeks. A moderate AMI requires gradual increase in activity after 4 weeks while a large AMI needs up to 6 weeks for recovery. During this time patients should avoid vigorous exertion and heavy lifting or activities which cause shortness of breath or sweating.

After initial recovery, patients are required to take medications to prevent a second AMI (ACC/AHA, 1996). These usually include aspirin, beta-blockers, ACE inhibitors, lipid lowering drugs, anticoagulants, and nitroglycerine. Additionally, patients 
are required to quit smoking, reduce weight and dietary fat consumption, control blood pressure and diabetes, reduce serum cholesterol and follow a regular prescribed exercise regime (ACC/AHA, 1996). These measures are known to improve quality of life and longevity after an AMI. Achievements of treatment goals can be facilitated through participation in formal cardiac rehabilitation program or home rehabilitation if patient is motivated (ACC/AHA, 1996). Cardiac rehabilitation combines prescriptive exercise training with education about coronary risk factor modification techniques. Formal rehabilitation programs have been shown to effectively improve functional capacity, promote compliance, decrease emotional distress, improve quality of life, reduce cardiovascular mortality, mitigate ischemic symptoms, promote reversal of atherosclerosis, and reduce risk of future coronary events.

\section{Beta-Blocker Therapy}

Beta-blockers exert an effect by blocking beta-receptors (Khan, 2000). Structurally they resemble catecholamines. Beta-blockers are competitive inhibitors, their action depending on the ratio of beta-blocker concentration to catecholamine concentration at the beta-receptor sites. Beta-receptors are sub-divided into two types:

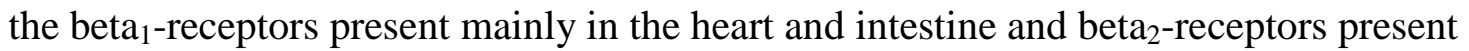
mainly in the bronchial and vascular smooth muscles. Thus, beta-blockers can be divided into cardioselective and non-cardioselective. Cardioselectivity implies that the drug blocks chiefly the receptors on the heart muscle and therefore partially spares the receptors on the lungs and blood vessels. However, selectivity may be lost at higher doses precipitating bronchospasm in susceptible individuals. Beta-blockers can also be 
divided based on the presence of intrinsic sympathomimetic activity (ISA). Intrinsic sympathomimetic activity (ISA) indicates partial agonist activity. Beta-blockers with ISA cause a slightly lower incidence of bradycardia compared with non-ISA drugs. However, this carries little or no advantages in angina at rest, or in angina at low exercise levels. This could, on the other hand, produce adverse effects on ventricular fibrillation threshold.

Physiologically, when used for AMI, beta-blockers rapidly reduce systolic blood pressure and there is reduction in cardiac output (Plosker \& Clissold, 1992). However, several weeks of therapy are required to achieve the maximum reduction in diastolic pressure and this reduction appears to result from a gradual decrease in total peripheral resistance (Benfield, Clissold, \& Brodgen, 1986). In addition to decreasing systemic arterial pressure, beta-blockers also decrease heart rate and contractility (Olsson, Ablad \& Ryden, 1990). They are known to reduce myocardial oxygen demand (ACC/AHA, 1996). Prolongation of diastole due to heart rate reduction probably facilitates blood flow through poorly perfused regions of the myocardium (Plosker \& Clissold, 1992). They delay cardiac ventricular repolarization, slow conduction velocity through the atrioventricular (AV) node, increase sinoatrial node recovery time and decrease automacity (Edvardsson \& Olsson, 1981; Camm, Ward \& Whitmarsh, 1982; Frishman \& Silverman, 1979; McDevitt, 1983). In accord with their electrophysiological effects, beta-blockers have shown antiarrhythmic activity in post myocardial infarction patients (Murray, Murray \& Little, 1986; Olsson \& Rehnqvist, 1984). They also involve prevention of the final thrombo-embolic process and/or progression of atherosclerotic lesions (Linden, Carmejo, Wilkund, Warnold, Olofsson \& Bondjers, 1988). A decrease 
in ejection velocity reduces hydraulic stress on the arterial wall, and this action may reduce the incidence of plaque rupture and thus protect from coronary thrombosis and fatal or non-fatal infarction (Khan, 2000). Beta-blockers may prevent early morning platelet aggregation induced by catecholamines, and decrease the early morning peak incidence of an AMI (Khan, 2000). They may also cause structural changes in plasma low density lipoprotein (LDL) resulting in a reduction of its potential for deposition in the arterial wall (Linden, Carmejo, Wilkund, Warnold, Olofsson \& Bondjers, 1988). These additional beneficial effects are believed to decrease the incidence of cerebrovascular complications and new myocardial infarctions, thus providing clinical efficacy.

Beta-blockers have certain side effects on the different systems it interacts with. Thus, in the cardiovascular system it can precipitate heart failure, atrioventricular (AV) block, hypotension, severe bradycardia, intermittent claudication, cold extremities, Raynaud's phenomenon and dyspnea. Dizziness, weakness, fatigue, vivid dreams, insomnia, and rare loss of hearing are central nervous system (CNS) side effects. Other side effects include nausea, vomiting, bronchospasm, respiratory distress, skin rashes, decreased libido and impotence (Khan, 2000).

\section{Effectiveness of Beta-Blockers for Post AMI}

Fifteen major randomized controlled trials of beta-blocker therapy administered after AMI were reported between 1974 and 1990 (Frishman \& Cheng, 1999). The mean follow-up time for these extended from 24 hours to six years. More than 18,000 patients with AMI were studied to document reductions in total deaths, cardiovascular death, coronary death, sudden death, and nonfatal re-infarction. The time between onset of AMI 
and initiation of treatment ranged from less than 24 hours to approximately 7.5 years. Patients with low and intermediate risk were studied in 12 of these trials whereas three trials involved patients with high risks. Results from 12 of the 15 long-term trials showed a lower mortality rate in the beta-blocker groups than the control groups. In three of the largest studies, the reduction in mortality rates was statistically significant, whereas in the remaining nine trials the results were not conclusive with regard to overall deaths. By combining the results of all 15 beta-blocker trials, a mortality rate reduction of approximately $21 \%$ was calculated. In eight trials that evaluated sudden cardiac death, lower rates were observed in the treatment groups. These trials yielded a trend of greater overall reduction in sudden cardiac deaths compared to all-cause deaths. An even larger benefit was recorded for instantaneous deaths, with an average reduction of $47 \%$. Ten of the 11 trials reporting the incidence of nonfatal re-infarction showed lower rates in the treatment groups compared to the control groups, however in only one of these trials, this was statistically significant.

In addition to these major randomized clinical trials, there have been various reviews and reports to study effectiveness of beta-blockers. A meta-analysis of about 65 studies, including long-term and short-term treatment with beta-blocker therapy was conducted by Yusuf et al., (Yusuf, Peto, Lewis, Collins \& Sleight, 1985). The overall results for the short-term trials demonstrated an effect on infarct size and on arrhythmias. However, a $6 \%$ risk reduction in mortality was found in the treatment group. This small difference did not reach statistical significance due to inadequate numbers in the randomization process. The long-term component included results of eight randomized trials, involving over 3,000 patients. Therapy was started orally a few days or weeks 
after myocardial infarction and continued for a period of some months or years thereafter. A crude overview of the results of these studies suggested a risk reduction of about $20 \%$. After weighting the average of the relative risks reduction, a pooled relative risk estimate of 0.77 was obtained. Though the trials had adequate numbers to demonstrate a main effect, it was not possible to select out sub-groups (defined by age, site, or severity of disease) of patients among whom therapy was advantageous and subgroups among whom it was not. To elucidate the mechanism by which death was prevented, deaths were classified as sudden and non-sudden based on time to death from the onset of pain. In several of the studies, reductions in sudden death were statistically significant with odds reduced by about $30 \%$. Not all trials reported the frequency of nonfatal re-infarction. Among those which did, treatment with long-term beta-blockers reduced the odds of reinfarction by about one-fourth and this was enormously significant $(\mathrm{P}<.0001)$. The metaanalysis concluded that long-term beta-blockade for perhaps a year or so following discharge after an AMI would reduce the odds of death and re-infarction by about $25 \%$. Differences among benefits of different beta-blockers were not apparent and side effects of therapy, being reversible by changing the beta-blocker or discontinuation of treatment, were not considered to be a major problem.

Hjalmarson conducted a review of data from five clinical trials that dealt with early and late interventions with beta-blockers following an AMI (Hjalmarson, 1988). Two of these trials, the Norwegian (NMS- Norwegian Multicenter Study) and the Goteborg, examined the effects of prophylactic use of beta-blocker therapy. The Norwegian trial had a 2-year follow-up with timolol as the beta-blocker and demonstrated a 36\% reduction in mortality. The Goteborg trial also demonstrated a 36\% reduction in 
mortality with metoprolol as the beta-blocker, however it involved a 3-month follow-up period. The American Beta-blocker Heart Attack Trial (BHAT) reported a 26\% reduction in mortality and involved propranolol as the beta-blocker. Effect of early intervention was also assessed in the Goteborg trial, which reported a 35\% greater reduction in mortality within the first two weeks. The other two trials in this review were Metoprolol In Acute Myocardial Infarction (MIAMI) and the First International Study of Infarct Survival (ISIS) trial. Both of these involved large numbers of patients, investigated early intervention and had lower differences in mortality as compared to the Goteborg trial in the range of $13-14 \%$. The reason mainly being that the trials included more low-risk patients, fewer older patients and fewer patients with pre-existing cardiac complications. The percent of patients withdrawn from these trials ranged from 19 to $29 \%$, and the reasons for withdrawal were presence of hypotension and/or bradycardia.

The above two reviews were restricted to reports from major trials. Goldstein reviewed 89 separate studies that addressed the effect of beta-blockers in heart disease generally (Goldstein, 1996). In these, 19 focused specifically on outcomes following an AMI. This review was more recent than the meta-analysis conducted by Yusuf et al., and reported average effects for late application of beta-blockers following AMI similar to those reported by Hjalmarson. In addition to overall mortality, this review reported reduction in cardiovascular mortality, re-infarction rate and sudden death due to betablocker use following an AMI. Data indicated that the beneficial effects of oral betablocker therapy were maintained for at least six years after an AMI, and discontinuation was associated with accelerated mortality. A trial involving late intervention in patients deemed high risk indicated a $48 \%$ decrease in overall mortality and a $58 \%$ decrease in 
vascular mortality. Analysis on subgroups identified by increasing mortality risk revealed mortality benefits from a low $2 \%$ to a high $23 \%$. Subgroup analysis was also conducted on data from trials, for high-risk patients such as those with congestive heart failure (CHF), elderly patients and patients with diabetes. Beta-blockers had a more marked effect in decreasing sudden death in those with CHF. It clearly illustrated that absolute benefits of treatment in older patients were greater than those achieved in younger patients. Additionally, for patients with diabetes the reduction in mortality was greater, (22-48\%) compared to patients without diabetes, (4-29\%). Thus, higher benefits accrue to patients who are at higher risk of mortality. It is important to consider the fact that in all of these studies the proportion of the AMI population determined to be eligible for beta-blocker therapy varied significantly in every study. Hjalmarson reported that 14$18 \%$ of patients were excluded from treatment in the Norwegian, Goteburg and the American trials because of contraindications. However, the specific contraindications were not mentioned in this review. Agusti et al., reported that $23 \%$ of the patients in their study had contraindications and these were history of chronic obstructive bronchitis, intermittent claudication, heart failure and permanent AV block (Agusti, Arnau \& Laporte, 1994). Another study, which mentioned specific contraindications, was by Whitford and Southern (Whitford \& Southern, 1994). The contraindications specified were 'active reversible airway disease, heart block, and heart failure'. This deemed 45\% of the patients ineligible for therapy.

An examination of the data from BHAT was done by Furberg and Byinton (Furberg \& Byinton, 1983) wherein they examined the difference in outcomes between patients in the tails of the distribution of expected mortality risk. They found a relative 
risk reduction of $40 \%$ in the group receiving beta-blocker agents in this limited set. Olsson et al., took a different approach (Olsson, et al, 1992). They collected individual patient level data from five randomized trials and analyzed the pooled data. They found a relative risk of mortality reduction of $19 \%$ overall. A higher benefit to women (23\%) was observed compared to men $(16 \%)$. The reduction in total mortality was mainly due to reduced sudden cardiac deaths. Additionally, beneficial effect of drug was not influenced by risk factors such as age, sex, and smoking habits. Thus, treatment effects were observed both in high and low risk patients. However, pooling data from different studies involves limitations, as each study involved different treatment protocols, dosages, treatment initiation and treatment duration time frames.

Beta-blockers have four potentially important ancillary properties: intrinsic sympathomimetic activity (ISA), beta ${ }^{-}$- selectivity, membrane stabilizing activity and lipophilicity (White, 1999). A meta-analysis of 73 trials was conducted to determine if these properties could help predict the degree of mortality benefit in the peri- and postAMI periods (Soriano, Meems, \& Grobbee, 1997). The results were divided among drugs with or without each of these properties. This meta-analysis suggested that the absence of intrinsic sympathomimetic effect and membrane stabilizing effect, and the presence of beta $1_{1}$ - selectivity and lipophilicity were most efficacious at reducing oneweek mortality, long-term mortality, re-infarction and sudden death. However, when the Cooperative Cardiovascular Project (CCP) data was investigated to compare effects of three beta-blockers- atenolol, metoprolol and propranolol, the study suggested that the specific beta-blocker selected has little influence on mortality (Gottlieb \& McCareter, 2001). The mortality rates of the lipophilic agent metoprolol and the nonlipophilic agent 
atenolol were virtually identical. When comparison was made between the nonselective agent propranolol with the selective agents- atenolol and metoprolol, propranolol had slightly negative outcomes in patients. However, patients receiving propranolol were sicker, compared to the other two agents, and after adjusting for the confounding variables, the difference between the selective and nonselective agents decreased. Furthermore, outcomes in patients who received propranolol was much better than patients not receiving any beta-blocker.

Observational studies involving retrospective database studies investigating betablocker efficacy have also been previously reported. A retrospective analysis was conducted to determine the effectiveness of metoprolol (a beta-blocker) for secondary prevention of AMI in Japan (Iskkawa et al, 2000). All AMI patients who were admitted or received regular outpatient treatment for AMI during an 11-year period from 1986 to 1996 were included. The primary endpoints were recurrent AMI (fatal and non-fatal), sudden death, or death from CHF. The metoprolol group had a 3\% incidence of cardiac events compared to the $6.8 \%$ of the non beta-blocker group, which represents a $56 \%$ reduction. Multivariate analysis revealed that metoprolol was significant in reducing cardiac events and in reducing the incidence of sudden death. Sub-group analysis by heart rate $(\geq 65$ and $<65$ beats/min) revealed that the effect of metoprolol on lower incidence of cardiac events remained unchanged.

Chen et al., investigated the data from the CCP, to determine whether betablockers were effective in reducing mortality after an AMI in patients who undergo coronary re-vascularization (Cheng, Radford, Wang, Marchiniak \& Krumholz, 2000). The CCP collected data on over 200,000 patients in 45 states over an eight-month period 
from 1994 to 1995 . The study was initiated due to the lack of inclusion of patients who undergo coronary re-vascularization such as PTCA or CABG in randomized clinical trials. Mortality at the end of one year between patients who underwent revascularization procedures such as $\mathrm{CABG}$ and PTCA and patients who did not undergo re-vascularization procedures were compared. The findings suggested that beta-blocker therapy was as effective in reducing-one year mortality for patients who undergo revascularization (CABG Vs. PTCA) as for patients not undergoing re-vascularization. Bisoprolol, a beta-blocker, has been shown to reduce cardiac death and myocardial infarction when used long-term in high-risk patients after major vascular surgery, further establishing the effectiveness of beta-blockers in this cohort of patients (Poldermans et al, 2001).

Due to their negative inotropic and chronotropic effects, beta-blockers are contraindicated in patients with pre-existing decompensated heart failure and acute pulmonary edema. However, they can be used in patients with large AMI that may result in left ventricular dysfunction (White, 1999). In a subgroup of Survival And Enlargement Ventricular (SAVE) trial, which evaluated patients with left-ventricular dysfunction after AMI, beta-blockers reduced the risk of cardiovascular death and the risk of developing severe heart failure (Vantrimpont, Rouleau, \& Wun, 1997). Thus, data suggests that patients with more severe AMI's and reduced ejection fraction after an AMI have greater benefits than those with less severe AMI's. In a subgroup analysis of the BHAT, patients with stable heart failure before receiving propranolol (a beta-blocker) achieved a mortality reduction similar to that of propranolol-treated patients without heart failure (Chadda, Goldstein, Byinton, \& Curb, 1986). Propranolol also reduced the 
occurrence of sudden death more frequently in patients with heart failure. Also, propranolol therapy did not increase the overall incidence of heart failure exacerbation, nor did it increase the incidence of heart failure exacerbation in patients with a prior history of heart failure.

Beta-blockers have shown to induce broncho-constriction in some patients with chronic obstructive pulmonary disease (COPD), hence COPD is a relative contraindication (White, 1999; ACC/AHA, 1996). However, it is usually patients with reversible obstructive lung disease (bronchial asthma, asthmatic bronchitis) who are at

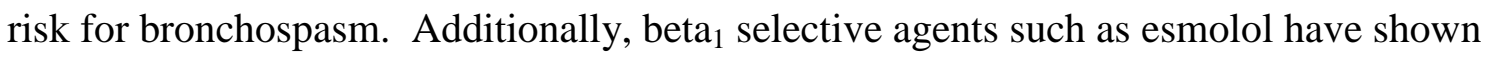
not to alter pulmonary function in patients with non-reversible obstruction (Gold, Dee, Cocca-Spofford \& Thompson, 1991). However, caution is required when used in such patients, as increase in dose may increase risk of worsening pulmonary function. Treating patients with fixed COPD with a beta s $_{1}$ selective agent poses great benefits. A retrospective trial using beta-blocker in patients with COPD revealed mortality risk reduction of 40\% (Gottleib, McCarter \& Vogel, 1998).

Beta-blockers are also relatively contraindicated in the patients with diabetes as they increase the risk for hypoglycemia (White, 1999). However, when used in diabetic patients during the post-AMI period, they have been associated with a $36 \%$ mortality risk reduction (Gottleib et al, 1998). This is important because patients with diabetes are historically known to have the worse outcomes compared to patients without diabetes, after an AMI. Thus, there is ample evidence that there are absolute benefits from betablocker therapy among patients with relative contraindications. Additionally, esmolol, a short-acting beta-blocker, has been demonstrated to be relatively safe in AMI patients 
with relative contraindications (Mooss, Hilleman, Mohiuddin \& Hunter, 1994). Thus, this drug can be replaced for conventional beta-blockers such as propranolol or metoprolol in such patients.

There has been debate about use of beta-blockers in patients with low-risk of complications associated with AMI (ACC/AHA, 1996). The risks of drug use could be greater than the benefits in these relatively healthy patients. However, a study including low-risk patients and beta-blocker therapy revealed a $40 \%$ decrease in mortality (Gottleib et al., 1998). Due to their better long-term prognosis, the absolute benefit in low-risk patients was not as great as high-risk patients, but there is a substantial improvement in survival.

\section{Recommendation for Beta-Blocker Use for Post AMI}

Due to their effectiveness, beta-blocker therapy has been included in the guidelines for management of patients with AMI by the ACC and AHA (ACC/AHA, 1996). The guidelines recommend their use both in hospital and for long-term prevention. The guidelines divide patients into three classes with respect to beta-blocker therapy - Class I, Class II and Class III. Class I include those patients with conditions for which there is evidence and/or general agreement that the therapy is beneficial, useful, and effective. Class II is sub-divided into IIa and IIb. Class IIa includes patients with conditions for which the weight of evidence/opinion is in favor of usefulness/efficacy of therapy and Class IIb includes patients with conditions for which usefulness/efficacy of therapy is less well established by evidence/opinion. And finally, Class III includes patients with 
conditions in the presence of which there is evidence and/or general agreement that a therapy is not useful/effective, and in some cases may be harmful.

For long-term therapy with beta-blockers, all patients accept those who are low-risk and without a clear contraindication to beta-blockers were included in Class I. Treatment for these patients should begin within a few days of the event and continue indefinitely. Low-risk patients without a clear contraindication to beta-blocker were in Class IIa. Class IIb did not include anyone. And Class III included patients with a contraindication to beta-blocker therapy. However, due to the increasing evidence of effectiveness of beta-blockers in patients with relative contraindications and low-risk patients, these guidelines were updated in 1999. According to the updated guidelines, Class I still includes patients who are not low-risk and have no clear contraindication to beta-blocker therapy. Class IIa includes low-risk patients without a clear contraindication to betablocker therapy and survivors of non-ST elevation AMI. There was an addition of Class IIb patients that included patients with moderate to severe left ventricular (LV) failure or other relative contraindications to beta-blockers, provided the patients were monitored closely. The updated guidelines did not include any patients in Class III.

In addition to being recommended by $\mathrm{ACC} / \mathrm{AHA}$, use of these agents long-term for secondary prevention after AMI has also been endorsed by other organizations such as the HEDIS (Health Plan Employer Data and Information Set). This organization has included long-term beta-blocker therapy as a quality of care indicator to evaluate performance of managed care plans (Bradford et al, 1999). 


\section{Utilization of Beta-blockers at Post AMI}

Although clinical trails have revealed impressive results, a large population of patients who could benefit from this therapy do not receive it in actual practice. Overall, $40 \%$ of all patients with AMI could be safely treated in the short-term with beta-blockers and at least $70 \%$ of patients could receive long-term therapy with beta-blockers (White, 1999). Surveys indicate that intravenous (IV) beta-blockers are used in $<15 \%$ of patients and oral beta-blockers are used in $<40 \%$ of patients without specific contraindications. Furthermore, $52-89 \%$ of patients in clinical practice receive beta-blocker doses that are $<50 \%$ of those studied in clinical trials.

The National Registry of Myocardial Infarction (NRMI) reported a 17 to $36 \%$ beta-blocker use in patients treated with thrombolytics and a 30 to $42 \%$ beta-blocker use in patients not receiving thrombolytics (Rogers et al, 1994). NRMI is a phase IV (post marketing), observational, collaborative endeavor sponsored by Genetec Inc, in which contributing hospitals throughout the United States record demographic, procedural, and outcomes data on patients with AMI. Even in 1996, in the quarterly report, the registry indicated that fewer than $50 \%$ of patients post AMI were taking beta-blockers (NRMI, 1996). However, a follow-up of this study in 1999 revealed a significant increase in betablocker usage at hospital discharge. This follow-up revealed that beta-blocker use increased from 42\% in 1994 to over $60 \%$ in 1999 (Rogers et al., 2000).

McCormik et al., examined utilization of beta-blockers in a different manner. They examined receipt of beta-blockers before hospitalization for recurrent AMI during 1986, 1988, 1990, 1991, 1993 and 1995 in all hospitals in Worcester, MA. (McCormik, Gurwitz, Lessard, Yarzebski, Gore \& Goldberg, 1999). They reported a moderate 
increase of beta-blocker use from $33.2 \%$ in 1986 to $44.4 \%$ in 1995 . Age and white race were negatively associated with beta-blocker use, whereas history of angina, hypertension, concurrent use of other cardiovascular medications such as aspirin and lipid-lowering drugs were positively associated with receiving beta-blocker agents.

Two recent studies that examined the consequences of beta-blocker under-use were conducted by Soumerai et al., and Krumholz et al. (Soumerai, McLaughin, Spielgelman, Hertzmark, Thibault \& Goldman, 1997; Krumholz, Radford, Wang, Chen, Heiat, \& Marciniak, 1998). The first study linked Medicare claims data to two databases covering pharmaceutical usage in New Jersey for these Medicare patients. Since the data utilized was for administrative claims, they were unable to identify all potential contraindications for beta-blocker therapy. Instead they defined eligibility as the absence of a diagnosis or medication reported prior to the AMI that would be suggestive of a contraindication. Thus, they excluded patients with CHF, asthma, COPD, as well as those with a prescription consistent with these diagnosis or a prescription for insulin. They found that $30 \%$ of the patients with AMI had 1 or more contraindications to betablocker treatment, thus $70 \%$ were eligible for therapy. However, only $21 \%$ of the eligible subjects received a prescription for beta-blockers. Also, among patients not receiving beta-blocker treatment before AMI, only $15 \%$ were started on therapy after the AMI. The second study collected data through an intensive chart review of Medicare AMI patients. These were selected from the CCP. For this study, Krumholz et al., excluded patients who died, were transferred, and who had contraindications for therapy. The contraindications included were: bradycardia, low blood pressure, high grade AV block, asthma, chronic lung disease, heart failure during hospitalizations or chart documented 
intolerance to beta-blocker. This study reported an eligible population of about 39\%. Of this, only $50 \%$ had a prescription for a beta-blocker at discharge.

Variation in beta-blocker usage has also been reported geographically. A significant variation in utilization by state and region was present in the CCP (Krumholz et al, 1998). Connecticut (77.1\%), Massachusetts (74.2\%), Maine (68.3\%), New Hampshire (68.9\%), and Vermont (66.7\%) had the highest utilization rates, whereas Mississippi (30.2\%), Puerto Rico (32.1\%), Oklahoma (33.5\%), Arkansas (33.5\%), and Nevada (36.4\%) had the lowest utilization rates. Also, among the different regions, New England had the highest utilization rates of $72.6 \%$. Variations within small areas were observed while examining data from six hospitals in Connecticut (Meehan, Hennen, Radford, Petrillo, Elstein, \& Ballard, 1995). Overall utilization rate was 41\%, however, it varied from $39 \%$ and $54 \%$ for the low-mortality hospitals to $29 \%$ and $33 \%$ for the high mortality hospitals.

Pilote et al., reported regional variation within the United States, when data from the Global Utilization of Streptokinase and Tissue plasminogen activator for Occluded coronary arteries trial (GUSTO-1) was explored (Pilote et al, 1995). Generally, they found that New England had the highest utilization of therapy with $71 \%$ of patients receiving a beta-blocker at discharge. Compared to this the Mountain and South Central regions had the lowest utilization at $47 \%$ and $49 \%$, respectively. The average age of patients in this study was between 59 and 62 for the various regions. An investigation of treatment and outcomes of AMI in Quebec from 1988-1995 revealed similar results (Pilote, Lavoie, Ho \& Eisenberg, 2000). Increasing beta-blocker utilization was found for the duration of the study, with the 50\% rate in 1995 being the highest. Analysis of 
patient treatment data in 11 geographically defined European regions revealed rates from $25 \%$ to $85 \%$ from center to center (Woods et al, 1998). About $48 \%$ of patients had at least one contraindication to beta-blocker on admission. In the patient group not admitted on a beta-blocker and without perceived contraindications, only $58 \%$ of patients actually received therapy at discharge. Variation in beta-blocker use has also been reported by urban-rural location. An examination of the CCP data in Kansas by rural, semi-rural and urban locations revealed utilization rates of $30.7 \%, 30.2 \%$ and $36.4 \%$, respectively, among ideal patients (patients who should definitely receive the agents). The trend was repeated even among the less than ideal patients (patients in whom use was controversial due to contraindications) with utilization rates of $22.5 \%, 25.0 \%$, and $31.3 \%$ in rural, semi-rural and urban locations (Sheikh \& Bullock, 2001).

Underutilization of beta-blockers, following AMI, has also been established in specific patient sub-groups. Fishkind et al., studied elderly patients (mean age $=81$ ) and found that utilization of beta-blockers following an AMI was quite low at 19\% (Fishkind, Paris, \& Aronow, 1997). This number is lower than the average usage in the overall AMI population. This suggests that physicians are reluctant to use this therapy in older patients, despite age not being a contraindication. Although age is not a contraindication, older patients are more likely to have conditions that will make them ineligible for therapy. Also, the authors included patients without consideration to contraindications. A recent study in the rural state of WV revealed similar results (Schade, Behm, Stephens \& Rezek, 2002). Around $59.4 \%$ of patients received beta-blockers at discharge over a span of six quarters with no detectable trend of improvement over time. Additionally, beta-blocker use declined with patient age. Gurwitz et al., extracted data from patient 
charts from 16 hospitals in a single Metropolitan Statistical Area for six years (Gurwitz, Goldberg, Chen, Gore, \& Alpert, 1992). They demonstrated a consistent trend toward reduced beta-blocker therapy in older patients. After adjusting for demographic and clinical variables (gender, prior history of angina, hypertension, or diabetes mellitus; myocardial infarction characteristics; complications including CHF and shock; and use of digoxin), odds ratio for receipt of beta blocker therapy relative to patients less than 55 years of age were 0.61 for those 55 to $64,0.52$ for those 65 to $74,0.36$ for those 75 to 84 , and 0.26 for those 85 or older. Utilization of therapy was $39.8 \%$ for the overall study population. Male patients and those with a prior history of angina or hypertension were more likely to receive therapy than those with diabetes mellitus.

Utilization differences have also been reported by gender and race. Pashos et al., conducted a retrospective analysis of administrative data (Pashos, Normand, Garfinkle, Newhouse, Epenstein, \& McNeil, 1994). This demonstrated odds ratios of 0.93 for women relative to men and 0.81 for blacks relative to non-blacks for receiving betablockers. Similar findings were reported while examining patient charts in two large urban hospitals (Sial, Malone, Freeman, Battiola, Nachodsky, \& Goodwin, 1994). A utilization of $38 \%$ was reported in patients with no potential contraindications. It reported a variety of conditions to be associated with under utilization of therapy. These include female gender $(\mathrm{OR}=0.52)$, health insurance $(\mathrm{OR}=0.34)$, presence of $\mathrm{COPD}$ $(\mathrm{OR}=0.21)$, development of $\mathrm{CHF}(\mathrm{OR}=0.46)$, and $\mathrm{AV}$ block $(\mathrm{OR}=0.28) . \mathrm{CHF}$ has been reported to be the reason for underutilization in a number of other studies. Also, presence of contraindications did not determine prescribing behavior. Up to $24 \%$ utilization was reported in patients with potential contraindications. Beta-blockers have certain adverse 
effects, which may also be responsible for it's under use. These include fatigue, depression, sexual dysfunction, nightmares and difficulty to detect hypoglycemia and diabetics.

The CCP data has also demonstrated association of socio-demographic characteristics with the use of beta-blockers (Rathore, et al., 2000). Beta-blockers were underused as discharge medications in the treatment of black, female and poor patients with AMI. This variation in treatment was not explained by severity of illness, physician specialty, hospital and geographic characteristics. Variation in beta-blocker use at discharge by gender was also reported by Wilkinson et al., in an observational follow-up study for 5 years from 1988 to 1992, with fewer women receiving therapy compared to men (Wilkinson, Laji, Ranjadayaln, Parson, \& Timmis, 1994).

Though there are reports of under utilization of these agents for the post AMI population, there has been a steady rise in average use of these agents over recent years. A recent study examined changes in post-AMI beta-blocker use occurring between 1994 and 1997 (Heller, Ahern, \& Kozak, 2000). It included patients $\geq 65$ years of age who were enrolled in Pennsylvania's pharmaceutical assistance contract for the elderly (PACE) and who survived AMI between 1994 and 1997. The results presented an increase of beta-blocker use from $39.6 \%$ in 1994 to $58.6 \%$ in 1997 . Those patients who did not use beta-blockers tended to have greater severity of illness, and co-morbidities such as CHF, COPD, and asthma. Other researchers have also pointed out strong time trends in the utilization of beta-blocker therapy. Paschos et al., (1994) documented a rise from $29 \%$ to $38 \%$ from 1988 to 1992 . Similarly Pilote et al., (2000) reported an increase from $33 \%$ to $50 \%$ from 1991 to 1995 . This may be due to publication of results of 
rigorous clinical trials. Recent survey indicates that beta-blockers are prescribed to as few as $40 \%$ of eligible patients in some health plans, however, there are some plans that report 96\% prescribing rates (NCQA, 1999; NCQA, 2000).

Most of the investigations associated with beta-blocker use have excluded patients with contraindications. However, a study which did examine utilization of beta-blockers in patients with contraindications revealed that $11 \%$ of patients with contraindications were receiving beta-blockers at discharge after an AMI (Brand et al., 1995). Some of these patients had absolute contraindications such as AV block, whereas some had relative contraindications such as diabetes mellitus, heart failure, and asthma. Another study, reported a $24 \%$ utilization in patients with potential contraindications such as $\mathrm{CHF}$, hypotension, cardiogenic shock, COPD, asthma, AV block and diabetes (Sial et al.,1994). Despite established guidelines there is ample evidence to establish under use of betablockers in patients with no contraindications and a few reports of misuse in patients with absolute contraindications. All these reports establish inappropriate utilization of betablockers in AMI patients for secondary prevention, and thus non-adherence to guidelines.

\section{Implications of Inappropriate Utilization of Beta-Blocker Therapy}

The inappropriate utilization of beta-blocker therapy raises the primary question "What are the consequences of this pattern of care?" There is very little information that addresses this aspect directly. There can be higher morbidity and mortality due to inappropriate care and there is a cost that can be attributed to these effects. Whenever effective treatment is withheld from a patient, or given to a patient not eligible, it can result in death or lower quality of life than anticipated. Each life lost or day of sub 
optimal health quality increases societal costs. In addition to having intrinsic value, every life saved also contributes to economic productivity. Second, there is the increase in medical resources utilized due to inappropriate care. This can be in the form of increased hospital readmission, frequent physician visits, increase in number of bed days and increase in emergency visits.

Lower mortality rates have been previously reported when there is utilization of beta-blockers. For example, Krumholz et al., reported a 14\% lower risk of mortality at one year after discharge, among eligible patients who received a prescription for betablockers at discharge, when compared to eligible patients who did not receive a prescription (Krumholz et al., 1998). Similarly, Soumerai et al., reported a 43\% lower mortality rate among beta-blocker recipients compared to non-recipients (Soumerai et al., 1997). Additionally, this study also reported that beta-blocker recipients were rehospitalized $22 \%$ less often than non-recipients were.

The costs associated with mortality has been addressed to some extent through simulation and modeling techniques for underutilization. One such study analyzed the cost effectiveness of routine use of beta-blockers in three specific ages of 45, 55 and 65 (Goldman, Sia, Cook, Rutherford \& Weinstein, 1988). Additionally, for each age group three prognostic categories were considered - high risk, medium risk and low risk. The estimated cost of six years of therapy to save an additional year of life was $\$ 23,400$ in low-risk patients, $\$ 5,900$ in medium-risk patients, and $\$ 3,600$ in high-risk patients for all the three age categories. This was assuming that the entire benefit of earlier treatment is lost immediately after six years. With a more likely assumption that the benefit of six years of treatment wears off gradually over the subsequent nine years, the results 
differed. Here the estimated cost of therapy per year of life saved was $\$ 13,000$ in lowrisk patients, $\$ 3,600$ in medium-risk patients, and $\$ 2,400$ in high-risk patients. The study disregarded costs savings generated in follow-up medical utilization or potential costs of any side effects.

Another study investigated health and economic benefits of increased betablocker use following an AMI. A computer simulated Markov-model of the coronary heart disease policy (CHDP) model was used in the US population (Philips et al., 2000). The aim of the study was to estimate the epidemiological impact and cost-effectiveness of increased beta-blocker use from current to target levels among survivors of AMI aged 35 to 84 years. Simulations included one cohort of AMI survivors in 2000 followed up for 20 years and 20 annual successive cohorts of all first-AMI survivors in 2000-2020. In the first group, this resulted in 4,300 fewer coronary heart disease (CHD) deaths, 3,500 fewer AMIs, and 45,000 life-years gained when utilization of therapy was compared to current use. The incremental cost per QALY (quality adjusted life years) gained was estimated to be $\$ 4,500$. For the second simulation, increase in use resulted in savings of \$18 million and 72,000 fewer CHD deaths, 62,000 fewer AMIs and 447,000 life years gained. Restricting the therapy to ideal patients however, would reduce the impact of beta-blocker therapy by about $60 \%$.

Thus, there has been some attempt to model effect of underutilization on patient outcomes. However, effect on utilization related variables such as physician visits, emergency room visits, length of stay and expenditures have not been investigated. Additionally, implications of use of beta-blocker therapy in patients with contraindications (misuse) has been ignored. Similar investigations in other chronic 
disease management suggests adverse patient outcomes due to misuse of therapy. For example, in asthma the National Heart, Lung and Blood Institute (NHLBI) developed guidelines for diagnosing and managing asthma (Podell, 1992). According to these guidelines, a short course of systemic corticosteroids is the most cost-effective way of reducing asthma excaberations. Long term $\mathrm{B}_{2}$ agonists and methylxanthines are recommended to be used as adjuvant therapy. Short-term $\mathrm{B}_{2}$ agonists should be used only on an as-needed basis. When drug therapy patterns and effect of these patterns on patient outcomes was investigated, the results demonstrated patterns outside of the guidelines (Shireman et al., 2002). Less than one-half of the population under study received sub-optimal dose per day of inhaled corticosteroid. A large percent (44\%) of the population received high or very high levels of short-acting $\mathrm{B}_{2}$ agonists. Additionally, patients with high doses of short-acting $\mathrm{B}_{2}$ agonists had the worst asthma outcomes. Their odds of receiving an oral steroid burst, an indicator of ambulatory asthma excaberations, were more than doubled. They were also more likely to be hospitalized and had significantly more emergency department visits. Another study which investigated effect of inappropriate medication use and outcomes in asthma was conducted by Anis et al. (Anis et al., 2001). This defined inappropriate medication use as use of nine or more canisters of short-acting $\mathrm{B}_{2}$ agonists, combined with no more than $100 \mathrm{ug} /$ day of corticosteroid in a year. Appropriate medication use was defined as four or fewer canisters of short-acting $B_{2}$ agonists and at least $400 \mathrm{ug} /$ day of corticosteroid. A greater proportion of those patients with inappropriate medication use were admitted to hospital on an urgent basis at least once during the year. Additionally, these patients were admitted or admitted on an urgent basis more frequently compared to the 
appropriate medication use group. The appropriate medication use groups had fewer physician visits overall and also fewer physician visits per physician. Thus, the inappropriately prescribed patients used more health care services, suggesting greater morbidity and greater health care costs.

Another class of drugs that have been investigated for inappropriate prescribing is antibiotics. An investigation of inappropriate antibiotic use in pyelonephritis revealed an association between inappropriate prescribing and length of stay (LOS) (Knapp, Knapp, Speedie, Yaeger, \& Naker, 1979). Inappropriate prescribing was associated with a twoday or $50 \%$ longer LOS than appropriate prescribing. This was regardless of disease severity. When a sub-analysis was conducted with the youngest cohort in this study, the inappropriately prescribed group still had a longer LOS, indicating that patient age did not affect inappropriate prescribing.

In addition to specific drug classes there have been investigations of multiple drug classes and inappropriate prescribing. Such investigations have examined inappropriate prescribing using criteria's such as indication, effectiveness, dosage, correct directions, practical directions, drug-drug interactions, drug-disease interactions, therapeutic duplication, duration and cost (Schmader et al., 1997). One such investigation revealed that patients with inappropriate prescribing were more likely to be admitted to the hospital or have an unscheduled physician visit during the subsequent 12 months. Lindely et al., identified inappropriate prescriptions, defined as unnecessary drugs and those with absolute contraindication, and adverse drug reactions (ADRs), defined as presenting symptoms that were known adverse effects of admission drugs, in 429 patients aged 65 years or over (Lindley, Tulley, Paramsothy, \& Tallis, 1992). Of the total ADRs 
presented, $72.8 \%$ were attributable to inappropriate prescriptions. Similarly, in a study of patients over 65 years, readmitted to the hospital, 17 of the 48 problems in 40 patients were drug-related (Bero, Lipton, \& Bird, 1991). These were overdose, under-dose, inappropriate choice, allergy and drug-drug interaction. Stroupe et al., investigated under and over supply of required medications using medication possession ratio in a network of community based ambulatory care centers (Stoupe et al., 2000). This revealed that patients with over supply had higher average inpatient and outpatient costs compared to patients with normal supply. Both under and over supply of medication were independently associated with significantly higher probabilities of hospital admission and emergency department visits.

Thus, there is evidence of inappropriate prescribing of various drugs and their consequent adverse health outcomes among patients. However, such investigations with beta-blocker use for post AMI patients for long-term prevention is non-existent, and needs to be undertaken.

\section{Physician's Role in Inappropriate Utilization}

A number of researchers have gone beyond the issue of beta-blocker utilization and attempted to understand the physician's role in beta-blocker therapy. As most of the research associated with beta-blocker utilization has investigated under-utilization, the physician's role has also been investigated in under-utilization more than inappropriate utilization. One of the primary findings is the association between beta-blocker prescribing and physician specialty. Fehrenbach et al. investigated an administrative managed care data retrospectively (Fehrenbach, Budnitz, Gazmararian, \& Krumholz, 
2001). The results revealed that $43.4 \%$ of family practice physicians, and $40 \%$ of other physicians prescribed beta-blockers compared to $67.7 \%$ by cardiologists. Others have documented prescribing rates of $77.5 \%$ for cardiologists, $63.0 \%$ for internists, and $53.1 \%$ for family practitioners of beta-blockers as post AMI therapy (Ayanian et al. 1994). The CCP data revealed prescription rates of $52.4 \%$ for cardiologists which was much higher than $39.7 \%, 35.4 \%, 36.1 \% \& 39.4 \%$ for internal medicine, family medicine, general practice and other specialty, respectively. In elderly patients, individuals who had prescriptions written by cardiologists were more likely to use a beta-blocker than individuals who received prescriptions from non-cardiologists $(\mathrm{OR}=1.52)$ ( Heller, Ahern, \& Kozak, 2000). A recent study in WV revealed similar results (Schade, Behm, Stephens \& Rezek, 2002). Around $59.4 \%$ of patients received beta-blockers at discharge over a span of six quarters with no detectable trend of improvement over time. However, there was a significant difference in beta-blocker use by physician specialty with prescribing rates of $60.8 \%$ by cardiologists, $49.7 \%$ by primary care practitioners and $45.8 \%$ by other specialties. All these suggest that physicians in different specialties are not equally aware of information and that this information difference may be a reason for differential prescribing rates by these physicians. Though cardiologists are more likely to prescribe beta-blockers at discharge, this does not necessarily imply that their prescribing behavior is adherent to guidelines. For example, a study measuring cardiologists' adherence to guidelines revealed a low level of compliance (Brand et al., 1995). About $31 \%$ of the cases had an error of omission (beta-blocker not given in the absence of a contraindication) and $11 \%$ of the cases had an error of commission (beta-blocker given in the presence of a contraindication). 
In addition to physician specialty there are other characteristics which have been shown to affect beta-blocker prescribing. For example Fehrenbach et al, revealed that $52.6 \%$ of physicians trained before 1980 prescribed beta-blocking agents as compared to 63.2\% physicians trained since 1980 (Fehrenbach et al, 2001). Additionally, region of practice was also associated with use of beta-blockers in this study, with the physicians in the northeast being more likely to prescribe beta-blocking agents than physicians in other regions. One other factor that affects prescribing is affiliation with university. Mitra et al., reported that patients at a government, university affiliated teaching hospital had high prescribing rates for beta-blocker therapy and this was in accordance with the ACC/AHA guidelines (Mitra, Findley, Frohnapple \& Mehta, 2002). This study examined the frequency with which cardiologists prescribed post AMI medications at discharges and evaluated medical management at the end of 24 months after discharge from the acute event. The study reported that over $90 \%$ patients were given beta-blockers at discharge, this utilization however decreased to $71 \%$ at the end of 2 years. These prescribing rates however, were much higher than the national registry rates of $48 \%$. Similar results were found when beta-blocker use was compared in two centers of Perth and Newcastle of world health organization (WHO). The study revealed that more patients were discharged from hospital on beta-blockers in Perth (68\%) than in Newcastle (45\%). The reason attributed to this difference was the fact that higher proportion of patients in Perth were treated by cardiologists in large teaching hospitals compared to the more rural nature of New-Castle (Nicholls, McElduff, Dobson, Jambrozik, Hobbs, \& Leitch, 2001). While the above studies have dealt with association between physician and physician practice characteristics and prescribing of beta-blockers, there are also reports 
of association between physician knowledge and beliefs with prescribing behavior. For example, cardiologists believed more strongly that beta-blockers improve survival as long-term therapy after an AMI and they were also more likely to prescribe this therapy compared to internists and family practitioners (Ayanian et al., 1994). In a critical look at literature regarding physician under-utilization of beta-blocker therapy, three primary reasons were revealed (Kennedy \& Rosensen, 1995). These include exaggerated concerns of adverse effects of beta-blockers, undue importance of contraindications, and skepticism of drug therapies due to competitive practice of pharmaceutical industry.

\section{Medicaid Program}

The present study will be undertaken in a Medicaid population. Thus, it is necessary to understand the structure and functioning of this health care system. Medicaid is a federal and state jointly funded health insurance program for the indigent, disabled, and members of families with dependent children (NPC, 1995). This program was started in 1965. In managing Medicaid, the federal and the state governments have separate responsibilities (NPC, 1995). The federal government provides fiscal assistance and a framework with regulations, guidelines, and policies for operation to state governments. State governments are responsible for administration of the Medicaid program which include - determining eligibility, determining services, claims processing and monitoring. Benefits provided by Medicaid include coverage for physician visits, inpatient and outpatient hospitalizations, laboratory tests, nursing home care, family planning services and supplies, and home health care. These benefits are mandated by the federal government. Additionally, there are optional services that are left to the 
discretion of each state. Some of these are coverage for pharmaceuticals, eyeglasses, and dental services for person 21 and older. Although optional, all states provide pharmaceutical coverage as a part of Medicaid benefits.

From the time of its initiation, Medicaid has had a tremendous impact on the US health care system. It has made healthcare accessible to individuals who are not financially well off. However, health care spending through Medicaid programs has grown tremendously. Total federal and state Medicaid expenditures, including administrative costs and dis-proportionate share hospital payments, increased from $\$ 58$ billion in 1989 to $\$ 194.7$ billion in 2000 (Coughlin, Ku, \& Holahan, 1994; Medicaid Consumer Information, 2003). One of the major reasons for this increase in expenditures is the increase in size of the Medicaid-covered populations. In 1990, there were 25.3 million recipients which increased to 36.3 million in 1995 and in 2000 there were more than 44 million (NPC, 1995; Medicaid Consumer Information, 2003).

This national trend for Medicaid growth is also evident in WV. This growth has been witnessed by both increase in enrollment and expenditures. In WV, the Bureau for Medical Services of the WV Department of Health and Human Resources has been responsible for the management of the Medicaid program (West Virginia Medicaid Program -WVMP). The total number of recipients in the WVMP has increased from 178,254 in 1982 to 354,326 in 2000 (NPC, 1998; Medicaid Consumer Information, 2003). This growth in number of recipients has resulted in a dramatic increase in expenditures from \$121 million in 1982 to over $\$ 1.391$ billion in 2000 (NPC, 1998; Medicaid Consumer Information, 2003). This can be interpreted as an increase in average spending per recipient from $\$ 678.84$ to approximately $\$ 3,900$ (NPC, 1998; 
Medicaid Consumer Information, 2003). Thus, it becomes important to identify areas that incur unnecessary costs - appropriate use of medication can be one such area. Investigation undertaken to study consequences of inappropriate care will help in developing and implementing strategies for minimizing expenditures for this health care system.

Thus, this chapter presented a review of literature associated with AMI, betablocker drug therapy, their effectiveness, recommendation for their use, variation in their use, implications of this variation in use and physicians role in this variation of use. It also provided a brief introduction to Medicaid, the health system in the study. The next chapter will discuss the methodology employed to fulfill the goals of this study. 


\section{CHAPTER THREE}

\section{METHODOLOGY}

This study had two phases. In phase I, the study evaluated the impact of appropriate and inappropriate prescribing of beta-blocker therapy by physicians, following an acute myocardial infarction (AMI), on patient outcomes and utilization of health services. In Phase II, the study determined physician-related factors that were related to beta-blocker prescribing in post-AMI patients. The methodologies required to accomplish the two phases were different. Phase I involved analysis of secondary data from paid claims of West Virginia (WV) Medicaid. Phase II was completed using primary data collected from physicians involved in post AMI care in the state of WV.

\section{Phase I}

The following sections describe the data source, data extraction, modification of raw data, and the analytical methods that were utilized for this phase of the study.

\section{$\underline{\text { Data Source }}$}

The WV Bureau for Medical Services (WVBMS) contracts with Consultec Inc. (Atlanta, Georgia), to serve as its claims processor. Consultec maintains and operates the Medicaid Management Information Systems (MMIS), which process provider claims and payments. MMIS data comprises of 3 files- provider, recipient, and claims files (Momani, 1999). 
The provider file contains specific information regarding various types of providers eligible to deliver services to Medicaid recipients. This includes a provider number, the provider's name, address, specialty, Medicaid eligibility, and tax related information. The recipient file contains information about Medicaid recipients, such as name, Medicaid number, eligibility begin date, end date, Social Security Number (SSN), aid category, gender, race and address. The claim files store detailed information specific to processed claims. For each medical claim information, such as invoice type, provider number, recipient number, International Classification of Disease $9^{\text {th }}$ edition (ICD-9) code of diagnosis for which service was provided, Common Procedural Terminology $4^{\text {th }}$ edition (CPT-4) code for procedures and services provided, Diagnosis Related Group (DRG) codes, date claims are submitted, date of adjudication through-date of service, coordination of benefit code, total primary carrier code, and total amount paid. For pharmaceutical products, the file contains fields such as name of the drug, begin date, number of days supply, metric quantity, National Drug Classification (NDC) code, generic code, therapeutic class code, refill number, pharmacy provider number, and the amount paid.

Paid claims data relevant for the study were obtained from Consultec, Inc. Consultec stores data going back several years. However, not all of this data were pertinent to this study and so relevant data between January 1, 1996 to June $30^{\text {th }}, 2001$ were extracted to conduct the analysis. 


\section{$\underline{\text { Data Extraction }}$}

The data obtained from Consultec was loaded on a computer in access files. These were extracted through the software BrioIntelligence and converted into statistical analysis software (SAS® version 8.2) data sets. The extraction process involved defining selection criteria, defining the time period of the study, inclusion of specific fields required, defining exclusion criteria, and classification of groups.

\section{Selection Criteria}

The main selection criteria for patients was recipient numbers who were discharged from a hospital with the primary or secondary diagnosis of AMI, ICD-9 codes 410.0 to 410.9, during January 1, 1997 to June 30, 2000. These recipient numbers were unduplicated to make sure that each recipient number occurred only once. Social security numbers (SSNs) for these recipients were identified. This is because SSN is the only unique identifier for Medicaid recipients since each recipient can have multiple Medicaid ID numbers.

\section{Time Period}

The total period of study was from January 1, 1997 to June 30, 2000 (42 months). From the database, every patient with AMI identified was categorized into two groups. The first group consisted of Class I \& II patients, who are eligible to receive beta-blocker therapy post-AMI and second group consisted of Class III patients, who are not eligible to receive beta-blocker therapy post-AMI. However, in order to do this, in addition to the data from the time period of the study, patient information from the previous year was also needed. Therefore, additional patient data for the time period of January 1, 1996 to 
December 31, 1996 was additionally extracted from the database. Also, since the study involved investigating outcomes for these AMI patients in the following one-year period after the AMI, additional data from July 1, 2000 to June 30, 2001 was extracted. Thus, the history file (hospital, ER, and physician), demographic file, managed care file, prescription or drug file were obtained for the identified AMI patients from January 1, 1996 to June 30, 2001.

\section{Specific Fields}

Appendix A provides the lists of the fields chosen that were essential for the study. From the demographic file, the fields selected were recipient number, recipient social security number (SSN), date of birth, sex, race, eligibility begin date and eligibility end date. From the hospital/ER file, the fields selected were recipient number, recipient SSN, first date of service, last date of service, billed amount, paid amount, procedure code, diagnosis codes and hospital extract indicator. From the physician file, the fields requested were recipient number, recipient $\mathrm{SSN}$, first date of service, last date of service, billed amount, paid amount, procedure code, diagnosis codes and physician extract indicator. From the pharmacy file, the fields requested were recipient number, recipient SSN, date of prescription filled, billed amount, paid amount, NDC code, generic code, and days supply. From the managed care file, the fields requested were recipient SSN, managed care begin date and managed care end date. The drug file had the NDC code, drug name and generic code. The DRG file had DRG code and DRG name. The procedure file had procedure (CPT-4) codes and code description. And finally, the diagnosis file had the diagnosis (ICD-9) codes and code description. 


\section{Exclusion Criteria}

The following exclusion criteria were applied to the selected SSNs with a diagnosis of an AMI during the time specified;

- The first restriction was to include only those patients who were continuously eligible in the Medicaid system for a period of one year before the hospitalized AMI under study.

- From these, managed care recipients were excluded as managed care recipients do not have all their utilization information in the Medicaid claims.

- Patients who were 65 years or older were excluded to avoid the issues of incomplete information as they have Medicare as their primary payer for health services. The age limit was set to 64 to avoid including those patients who would become eligible for Medicare during the period of follow-up after being initially present.

- Patient who have length of hospital stay less than 3 days were excluded to avoid error due to misdiagnosis of AMI.

- Patients with the primary ICD-9 code of 410.x2 were excluded as this code is utilized for subsequent episodes of care for AMI where the initial treatment was received less than 8 weeks ago.

- When patients who had recurrent AMI within the time period of the study were present, only the first AMI was considered.

- Beta-blocker receipt in the study was defined as presence of beta-blocker prescription in the claims within 90 days of discharge. Thus, those patients who did not receive a prescription for beta-blockers in the first 90 days after the AMI but 
received it sometime within the year were excluded to prevent potential bias due to misclassification.

- Patients who died during the AMI or within 30 days of the incident AMI were excluded.

The resultant group of patients was investigated to see the effect of appropriate and inappropriate prescribing on mortality

- Patients who died due to non-cardiac causes in the follow-up period were excluded.

The resultant group of patients was investigated to see the effect of appropriate and inappropriate prescribing on cardiac mortality

- Patients who were not continuously eligible for Medicaid in the follow-up oneyear period after the AMI were excluded.

The resultant group of patients was investigated to see the effect of appropriate and inappropriate prescribing on health care utilization and expenditures. This process of applying inclusion and exclusion criteria is outlined in Figure 4. 
Figure 4: Data Extraction

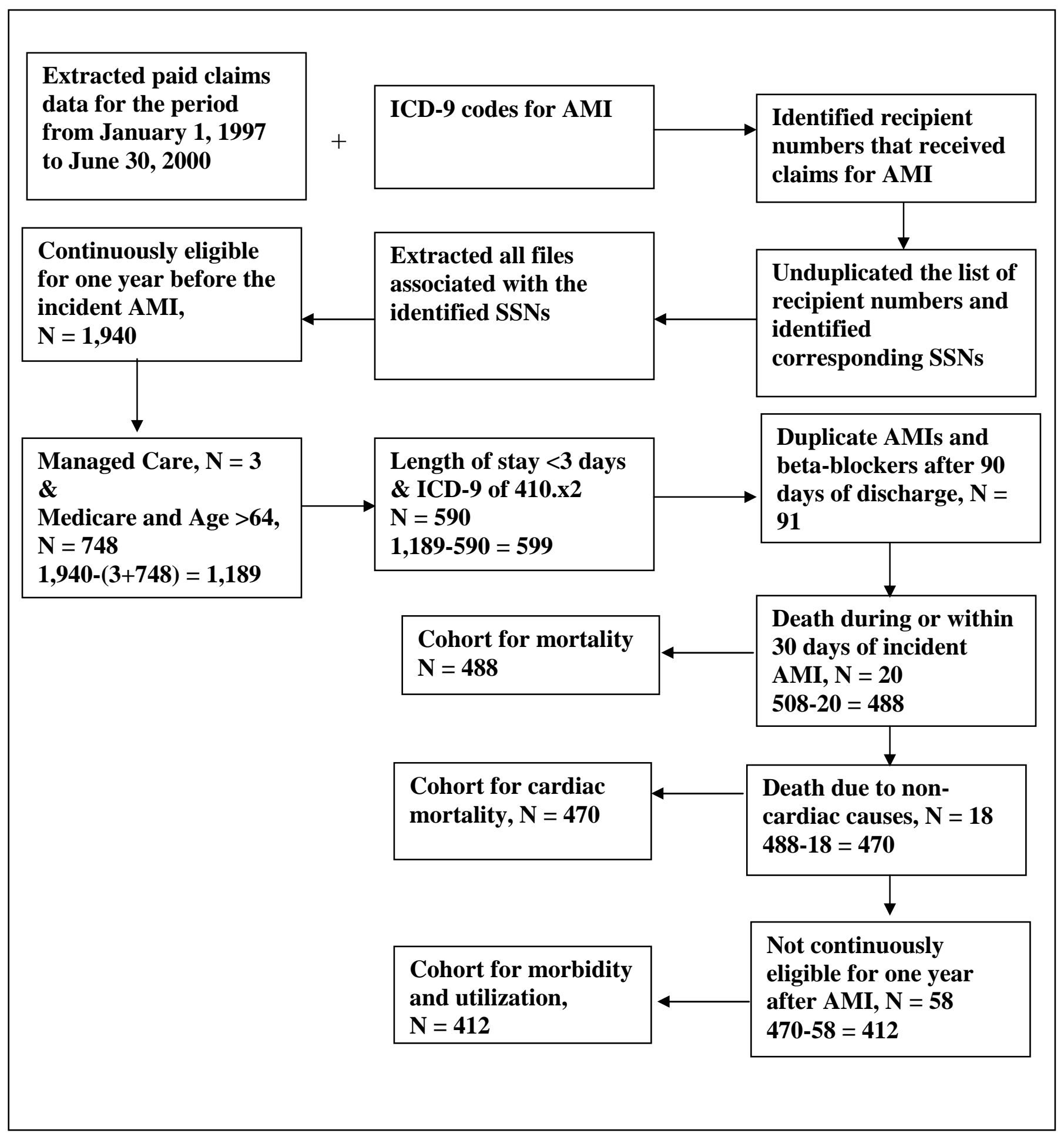

AMI: Acute myocardial infarction, ICD-9: International classification of disease- $9^{\text {th }}$ edition, SSN: Social security numbers 


\section{Classification of groups}

Information about procedures, services, medications and medical history were extracted during the incident AMI, one year prior to the incident AMI and one year after the incident AMI for every patient identified who satisfied all the inclusion and exclusion criteria. Based on their medical history, patients were divided into those with absolute contraindications, and those with relative contraindications, using ICD-9, CPT-4 codes and DRG-codes (see Appendix B). For example, a person with the ICD-9 code of 458.x was considered to have hypotension, an absolute contraindication. It is important to point out that certain relative contraindications such as a heart rate less than $60 \mathrm{bpm}$, systolic arterial pressure less than $100 \mathrm{Hg}$, signs of peripheral hypoperfusion, and beta-blocker intolerance were not recognized and constitute a limitation of this study.

Based on presence of the contraindications, patients were classified as Class I, II and III for beta-blocker use after AMI. Prescription claims within 90 days of AMI were obtained. Patients were classified into two groups based on presence of absolute contraindications and beta-blocker receipt. Those who did not have absolute contraindications and received therapy and those who had absolute contraindications and did not receive therapy constituted the appropriately prescribed group. Similarly, those who did not have absolute contraindications and did not receive therapy and those who had absolute contraindications and received therapy constituted the inappropriately prescribed group. Additionally, a sub-classification within the inappropriately prescribed group was created to differentiate between patients with under use (those who did not have absolute contraindications and were not prescribed) and misuse (those who had absolute contraindications and were prescribed) of beta-blocker therapy. 


\section{Modification of Raw Data}

The raw data obtained from the hospital, physician and prescription claims data was modified to obtain all the relevant variables that were required for the analysis, using SAS® version 8.2. For the pre-phase (before the incident AMI) and during the incident AMI, information about various medical conditions, such as left ventricular (LV) failure, $1^{\text {st }}$ and/or $2^{\text {nd }}$ and /or $3^{\text {rd }}$ degree atrioventicular (AV) block, chronic obstructive pulmonary disease (COPD), asthma, insulin dependent diabetes mellitus (IDDM), hypotension, bradycardia, non-insulin diabetes mellitus (NIDDM), hypertension, previous $\mathrm{AMI}$, previous coronary artery bypass graft $(\mathrm{CABG})$, previous percutaneous transluminal coronary angioplasty (PTCA), congestive heart failure (CHF), stroke and renal dysfunction was obtained. The presence of these were determined by presence of either ICD-9 codes or CPT-4 codes in either the hospital or the physician claims during the year before the incident AMI or as secondary diagnosis during the incident AMI. In some cases DRG-codes were used (only CABG and PTCA). These are listed in Appendix B.

The hospital claims had both hospitalization and ER claims in one file and the physician file contained claims for clinic, department of health screening, lab tests, transportation, family planning, special services and practitioner. Thus, the hospitalization file required to be separated into hospitalizations and ER visits and the physician file had to be limited to only outpatient physician visits. There was a variable called hospitalization extract indicator in the hospital claims data, which classified the claims as hospitalizations or ER visit. This variable and the following criteria were utilized to separate the hospital claims data into hospitalization or ER visits. Those 
claims that had the variable as 1 (indicating hospital claim), had length of stay greater than 1 day, and had a specific DRG code were classified as hospitalizations. Those events which had length of stay as zero, had the indicator variable as 2 (indicating ER extraction) and CPT-4 codes between 99281 to 99285 (CPT-4 codes for emergency department visits) were classified as ER visits. There were claims that did not fit into both these categories. An inspection of the procedure codes of these claims revealed that these were claims associated with laboratory, radiology, \& pathology during either hospital admissions or ER admissions. Since the only information needed from these claims were billed and paid amounts, they were classified as hospital/ER laboratory claims. Similarly, in the physician claims files there was an extract indicator variable, which was utilized to classify the physician visits as outpatient physician visits and outpatient laboratory claims. All claims were converted to one observation per visit. However, the billed and paid values for these observations were a sum of all the billed and paid values of all the claims associated with that visit.

The total number of admissions for any cardiac condition in the pre-phase was determined by summing the number of hospitalizations in the pre-phase with ICD-9 codes for cardiac conditions (Appendix B). Similarly, those hospitalizations that were not related to cardiac conditions were summed to get total number of admissions due to non-cardiac conditions in the pre-phase.

The pharmacy claims data was combined with the drug file to get drug names in the database. Based on drug names, the total number of different unique drug claims per person in the six months before the incident AMI was utilized to get total number of drugs in the pre-phase. Similarly, any use of drugs such as digoxin, Angiotensin 
converting enzyme (ACE) inhibitors, anti-arrhythmic agents, beta-blockers, and calcium channel blockers in the pre-phase was determined. Use of these agents in the 90-day period after the incident AMI was also determined. Medication possession ratio and total days supply of beta-blockers in the post-AMI period was determined. Medication possession ratio is defined as

Total days supply of medication during study period Total number of days between fill date of first and last prescription + days supply on last claim

Total days supply was the sum of the days for which the patient filled a prescription during the year after the incident AMI. In the post-AMI period, the total number of hospitalizations, ER visits, physician visits and total length of stay for those who had hospitalizations for the cardiac conditions under study were estimated by addition of each visit for the specific ICD-9 codes (See Appendix B-outcomes conditions).

Expenditures were defined as direct costs incurred to Medicaid. Thus, reimbursed amount was obtained by summing the paid amounts for the specific ICD-9 codes from the hospitalizations, ER visits, hospital/ER laboratory, physician visits and physician laboratory files for each patient. Similarly, total charges was obtained by adding the billed amounts for the specific ICD-9 codes from the hospitalizations, ER visits, hospital/ER laboratory, physician visits and physician laboratory files for each patient.

\section{$\underline{\text { Research Design }}$}

The research design used for this phase of the study was a quasi-experimental longitudinal design. There was an experimental group and a comparison group, i.e. the appropriately prescribed group and the inappropriately prescribed group. However, since 
the allocation to group was not randomized it was a quasi-experimental design. Both the groups were followed for a period of 12-months to determine the effect of prescribing on outcomes, thus it is also longitudinal. Additionally, since the study involved following a cohort of patients, determining their exposure to inappropriate/appropriate beta-blocker use and determining the effect of this exposure on outcomes, it can also be classified as a cohort design. 
Figure 5: Quasi-experimental Longitudinal Research Design

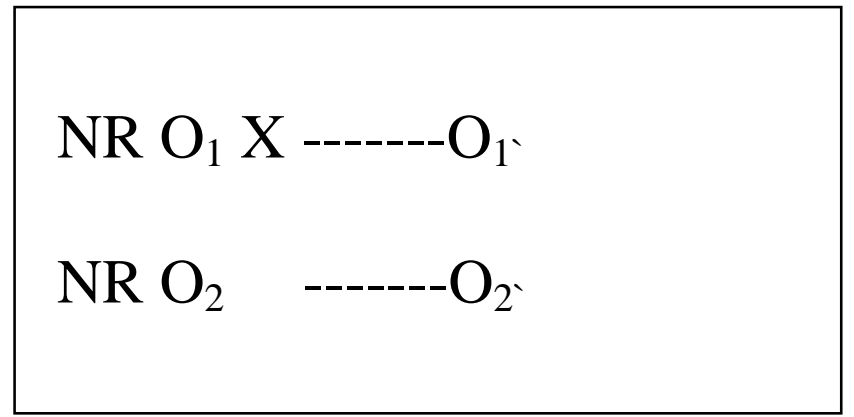

NR: Non randomized, $\mathrm{X}$ : Exposure, $\mathrm{O}_{1}$ : Experimental group, $\mathrm{O}_{2}$ : Control group 


\section{Data Analysis for Phase I}

The data analysis used for this phase is presented by research questions proposed. The Statistical Package for Social Sciences® (SPSS) version 10) was used for data analysis:

\section{Research Question 1: Prescribing Patterns for Beta-blocker Therapy in WV Medicaid}

Frequencies were calculated to identify what proportion of patients received betablocker therapy, appropriately and inappropriately, as per AHA/ACC guidelines. There were four classes of patients; those who should be prescribed and received a prescription - appropriate use, those who should be prescribed and did not receive a prescription under use, those who should not be prescribed and received a prescription - misuse and those who should not be prescribed and did not receive a prescription - appropriate use. The percentage of patients in the misuse category was low, and hence was pooled with the under use category to get an inappropriately prescribed group which was utilized for analysis purposes. Similarly, the two appropriate use groups were pooled into one.

These two groups (appropriate and inappropriate) were compared on demographic and patient history (co-morbidities and drug use) characteristics with t-tests and chisquares depending on whether the variable was dichotomous or categorical. The different characteristics compared were: age, gender, presence of diabetes, hypertension, peripheral vascular disease, renal dysfunction, previous AMI, previous $\mathrm{CHF}$, previous stroke, previous $\mathrm{CABG}$, presence of relative contraindications, presence of absolute contraindications, number of cardiac admissions in the year prior to AMI, number of noncardiac admissions in the year prior to AMI, number of different drugs in the six months 
before AMI, CABG during incident AMI, PTCA during incident AMI, length of stay during incident AMI, number of secondary diagnosis during incident AMI, use of digoxin, ACE inhibitors, anti-arrhythmics, beta-blockers and calcium channel blockers before the incident AMI.

Of these, age, hypertension, presence of absolute contraindications, number of non-cardiac admissions, use of digoxin and use of beta-blockers in the pre-period were significantly different between the two groups. These variables were used in a logistic regression to predict group status. Group 1 was for appropriately prescribed patients and 0 for inappropriately prescribed patients. The predicted value of this logistic regression was used as propensity score for further analysis. Propensity scores calculated from observational characteristics of patients are used to reduce selection bias in observational studies. This is because in observational studies, investigators have no control over inherent risks that patients bring into the study. Thus, large differences on observed covariates in the selected groups, may lead to biased estimates of outcomes. Propensity score calculated from these observed co-variates can thus be used to reduce bias and increase precision.

The equation for the logistic regression can be represented as:

Appropriate assignment $(\mathrm{Y})=\quad \mathrm{X}_{1}$ (age)

to treatment with beta-blockers $\quad+X_{2}$ (hypertension)

$+\mathrm{X}_{3}$ (presence of absolute contraindications)

$+\mathrm{X}_{4}$ (number of previous non-cardiac admissions)

$+\mathrm{X}_{5}$ (previous use of digoxin)

$+\mathrm{X}_{6}$ (previous use of beta-blockers) 
Research Question 2: Effect of Appropriate and Inappropriate Prescribing on Mortality

Patients who did not have follow-up information and were confirmed to have died from death certificate records in the 12-month period after the AMI were utilized to study the relationship between appropriate and inappropriate prescribing of beta-blockers and mortality. This relationship was investigated by Kaplan-Meier survival curves where the dependent variable was time to death and the independent variables were appropriate and inappropriate use. A duplicate analysis was conducted with time to cardiac death as the dependent variable. Significant survival analysis is usually followed by Cox-proportional hazards regression model, where the effect of the main independent variable on survival time is examined after adjusting for other covariates. However, since the survival time for cardiac death was not significant, a follow-up Cox-proportional hazards model was not conducted.

The equations for the survival analysis can be represented as follows;

\section{Survival Analysis:}

Time to death $(\mathrm{Y})=\mathrm{X}_{\mathrm{i}}($ appropriate use $)+\mathrm{X}_{\mathrm{ii}}$ (inappropriate use)

Where, $\mathrm{X}_{\mathrm{i}}$, was 1 for those patients in the appropriate group and $\mathrm{X}_{\mathrm{ii}}$ was 1 for those patients in the inappropriate group.

\section{Research Question 3: Effect of Appropriate and Inappropriate Prescribing on Morbidity}

Patients who had follow-up data and were continuously eligible for the follow-up period were utilized to investigate the relationship between beta-blocker prescribing and morbidity. As in the case of mortality, Kaplan-Meier survival curves were utilized to examine the relationship between cardiovascular $(\mathrm{CV})$ morbidity and prescribing of beta- 
blockers following an AMI. However, the dependent variable was time to event during the follow-up period where event was first hospitalization due to any of the following: a successive AMI, heart failure, stroke, angina, other ischemic disease, essential hypertension, cardiogenic shock, disease of endocardium, conduction disorders and/or cardiomyopathy, in the follow-up period after the incident AMI. Since survival analysis was found to be significant it was followed by a Cox-proportional regression model to adjust for the various co-variates. Thus the equations were;

\section{Survival Analysis:}

Time to event $(\mathrm{Y})=\mathrm{X}_{\mathrm{i}}($ appropriate use $)+\mathrm{X}_{\mathrm{ii}}$ (inappropriate use $)$

\section{Cox-Proportional Hazards Regression Model:}

Time to event $(\mathrm{Y})=\mathrm{X}_{1}($ demographics- age, gender $)$ $+\mathrm{X}_{2}$ (appropriate/inappropriate use) $+\mathrm{X}_{3}$ (predictors of appropriate beta-blocker assignment or propensity score) $+\mathrm{X}_{4}$ (presence of absolute contraindications) $+\mathrm{X}_{5}$ (current procedures CABG, PTCA, length of stay) $+\mathrm{X}_{6}$ (other discharge medications - aspirin, calcium channel blockers, ACE inhibitors, loop diuretics) $+\mathrm{X}_{7}$ (medication possession ratio) $+\mathrm{X}_{8}$ (days supply)

Where $\mathrm{X}_{1}$, represented demographic characteristics such as age and gender. 
$\mathrm{X}_{2}$, was 1 for those patients who were in the appropriately prescribed group and 0 for those who were inappropriately prescribed group.

$\mathrm{X}_{3}$, was propensity score estimated through the logistic regression with group status as the predictor, estimated in research question 1. An alternative analysis was done where this included a list of variables that were significant predictors of appropriate betablocker receipt in the logistic regression for predicting group status in research question 1. $\mathrm{X}_{4}$, was 1 if any of the absolute contraindications such as Bradycardia, Hypotension, and/or AV block were present and 0 if none of them were present. $\mathrm{X}_{5}$, and $\mathrm{X}_{6}$, were variables associated with procedures undertaken during the incident AMI and discharge medications after the incident AMI, respectively. $\mathrm{X}_{7}$, was medication possession ratio. Medication possession ratio is proxy for patient compliance calculated as days of supply of the medication divided by the total days elapsed between $1^{\text {st }}$ fill and last fill of the medication, which was obtained from the prescription data.

$\mathrm{X}_{8}$, was days supply a total of number of days for which the patient had beta-blockers filled.

Research Question 4: Effect of Appropriate and Inappropriate Prescribing on Utilization and Expenditure

Patients who had follow-up data and were continuously eligible for the follow-up period were utilized to investigate the relationship between beta-blocker prescribing and health care utilization and expenditure. To examine the relationship between prescribing 
of beta-blockers and utilization of health care services, multiple regression analyses were conducted. The utilization variables investigated were:

$$
\begin{aligned}
& Y_{1}=\text { Number of physician visits due to cardiac conditions } \\
& Y_{2}=\text { Number of hospitalizations due to cardiac conditions } \\
& Y_{3}=\text { Total hospital length of stay due to cardiac conditions } \\
& Y_{4}=\text { Number of ER visits due to cardiac conditions }
\end{aligned}
$$

Cardiac conditions included the following: a successive AMI, heart failure, stroke, angina, other ischemic disease, essential hypertension, cardiogenic shock, disease of endocardium, conduction disorders and/or cardiomyopathy, in the follow-up period after the incident AMI. To examine the relationship between prescribing of beta-blockers and expenditures, two multiple regression analyses were conducted. The dependent variables were:

$\mathrm{Y}_{5}=\mathrm{Log}$ total charges associated with physician visits, hospitalizations, and ER visits due to cardiac conditions $\mathrm{Y}_{6}=\mathrm{Log}$ total reimbursed amounts associated with physician visits, hospitalizations, and ER visits due to cardiac conditions Here, cardiac conditions were the same as defined earlier. Reimbursed amount and charges were transformed into log form to decrease effect of skewness associated with the cost data. The independent variables were similar to the regression models for morbidity analysis. Thus, the generic equation can be represented as; 


$$
\begin{array}{ll}
\text { Dependent variable }(\mathrm{Y})=\quad & \mathrm{X}_{1} \text { (demographics- age, gender) } \\
& +\mathrm{X}_{2} \text { (appropriate/inappropriate use) } \\
& +\mathrm{X}_{3} \text { (predictors of appropriate beta-blocker } \\
& \text { assignment or propensity score) } \\
& +\mathrm{X}_{4} \text { (presence of absolute contraindications) } \\
& +\mathrm{X}_{5} \text { (current procedures CABG, PTCA, length of } \\
& \text { stay) } \\
& +\mathrm{X}_{6} \text { (other discharge medications }- \text { aspirin, calcium } \\
& \text { channel blockers, ACE inhibitors, Loop diuretics }) \\
& +\mathrm{X}_{7} \text { (medication possession ratio) } \\
& +\mathrm{X}_{8}(\text { days supply) }
\end{array}
$$

\section{Phase II}

The goal of the second phase was to determine association between physician characteristics and prescribing behavior for beta-blockers for secondary prevention following an AMI. For this, knowledge of contraindications, willingness to prescribe, socio-demographic, and practice characteristics of prescribers in the state were obtained. Primary data was collected for this phase using a survey instrument. The following section describes the research design, methodology for identifying the study population, development of the survey instrument, the data collection process, and data analytical techniques that were used for this phase of the study. 


\section{$\underline{\text { Research Design }}$}

The research design employed for this phase of the study was a cross-sectional design. The assumption made for this phase is that the knowledge, willingness to prescribe and socio-demographic and practice characteristics of physicians would affect their prescribing behavior. In this phase of the study, the dependent variable was prescribing rate of beta-blocker therapy by physicians, which was obtained from the survey.

\section{Study Population and Sample Selection}

The population of interest for this phase was physicians who were care-providers for AMI patients in WV. A mailing list of all WV board certified physicians in the specialties of cardiology, internal medicine, primary practice and general practice was obtained from the West Virginia Medical Board (WVMB). The total number of physicians in the state obtained from the WVMB in these specialties was 1151. Since physician surveys are associated with low response rates, all of the listed physicians were surveyed.

\section{Instrument Development and Content}

A self-administered survey was used for this phase. Mail surveys offer many advantages such as the ability to collect data from a larger geographical area at a relatively low cost, greater versatility, and absence of interviewer bias. For respondents, it offers the flexibility of replying at their convenience and offers respondent anonymity. In developing the survey instrument, attention should be given to the kind of information 
needed to measure both the independent and dependent variables of interest, length of questionnaire, cost, comprehension level of prospective respondents, complexity of the questionnaire and the time required to complete it (Dillman, 1978). All these factors collectively can have a significant impact on the kind of data collected and the response rate.

The survey in this study was designed to assess the WV physicians' knowledge of contraindications according to the guidelines associated with post AMI care and use of beta-blockers, and their willingness to prescribe beta-blocker therapy in patients with various co-morbidities. To avoid leading the respondents to desirable responses, questions for other post-AMI medications were included in the survey. Thus, section one of the survey was designed to be more general for post-AMI medications. The first question asked the respondents to mark those medications which were important secondary preventive agents following an AMI. The response set included those medications which are present in the AHA/ACC guidelines. A follow-up question required the respondents to rate their willingness to prescribe these medications for patients after an AMI, on a 1 to 5 Likert scale where 1 was least likely and 5 was most likely. The next two questions in this section asked respondents to indicate how many of the last 10 AMI patients they had treated, they had prescribed beta-blockers and ACE inhibitors. Those respondents whose response was less than 10 were instructed to list the specific reasons for not prescribing these medications in those patients not prescribed beta-blockers or ACE-inhibitors.

The second section of the survey was primarily designed to measure willingness to prescribe beta-blockers in patients with other co-morbidities, on a 1 to 5 Likert scale 
where 1 was least likely and 5 was most likely. The respondents were also asked to list their willingness to prescribe ACE-inhibitors in these patients with other co-morbidties. However, this was done to prevent leading the respondents into giving desirable responses to questions with regards to beta-blocker use. The co-morbidities included a list of conditions that were either relative or absolute contraindications for beta-blocker use, or conditions that are associated with higher or lower risk of complications, during an AMI. A few other conditions associated with CV disease, but not associated with beta-blocker use, were also added to give a better mix of co-morbidities.

The third section of the questionnaire focused on knowledge of contraindications. The respondents were asked to classify a list of conditions into relative and absolute contraindications and conditions that were neither relative nor absolute contraindications, for use of beta-blockers after an AMI. The response set consisted of a list of conditions, which are either relative or absolute contraindications for beta-blocker use. A few conditions, which were neither relative nor absolute contraindications, were also listed to give a good mixture of disease conditions. These conditions were listed randomly in the response set.

Socio-demographic and practice information were obtained in the last section of the survey. Data obtained included age, gender, specialty, year of board certification in this specialty, the average number of patients with AMI they treat per month (both new and repeat), the number of beds in the hospital which is their primary practice site, and information about their primary practice site. The demographic questions were asked on a dichotomous or an interval scale. Open-ended questions were used for obtaining information about practice characteristics. 


\section{$\underline{\text { Instrument Validation }}$}

The instrument was submitted to experts for face and content validation. Experts in the area of health services research at West Virginia University were approached to assess clarity and appropriateness. Content validity is defined as " the extent to which test exercises reflect and fully cover the curriculum which the test was designed to measure" (Mussio, 1987). Feedback from the experts was incorporated into the survey. Institutional review board (IRB) permission was obtained from West Virginia University on the validated survey. The final survey is represented in Appendix F.

\section{Instrument Pilot Testing}

The survey instrument was pre-tested to assess clarity, readability, and time for completion. This process helps determine whether any important variable is being omitted, any information is redundant, and if any changes are required in the questionnaire wording and formatting, determine the ease of use and comprehension of the instrument, and helps obtain a mean completion time. Pilot testing was conducted in a convenience sample of cardiology, and internal medicine residents at the West Virginia School of Medicine. A total of six internal medicine residents and two cardiology residents responded to the survey. Seven of these surveys had complete responses to all the questions indicating ease of comprehension. The residents were asked to rate the questionnaire for clarity and readability on a 1 to 5 scale where 1 was unacceptable and 5 was acceptable. A total of 5 residents responded to this question, and the mean was 5 for both the clarity and readability. The residents were also asked to state the time taken for completing the survey. Five residents responded to this question and the mean time for 
completion was 11.4 minutes. No changes were made to the survey and the pilot testing suggested that the survey was suitable for the study.

\section{$\underline{\text { Data Collection }}$}

The data collection process included survey implementation, and non-response assessment.

\section{Survey Implementation}

Physicians' from the mailing list were mailed an individualized cover letter, the survey instrument and a business reply envelope. The cover letter explained the purpose of the study, and assured confidentiality of response (Appendix C). Respondents were asked to return the completed questionnaire in the self-addressed business reply envelope

provided in the package. The surveys were coded for follow-up purposes. All completed and returned questionnaires were checked off the mailing list. A second mailing was made two weeks after the first one. This consisted of the questionnaire survey, the second cover letter, and the self-addressed business reply envelope. The second mailing was done only to those physicians who did not respond to the first mailing. This was possible due to the coding procedure utilized during the first mailing. The second cover letter was designed to stress the importance of participating in the study (Appendix D). Similar to the first mailing, completed responses were checked off the coding list. The physicians who did not respond to the second mailing were sent a third mailing to boost the response rate. This package contained the third cover letter (Appendix E), which 
stressed the importance of the physicians' response, the survey instrument and the selfaddressed envelope. All three cover letters were pre-approved by the IRB.

\section{Non-Response Survey}

Bias can be introduced into the study due to possible differences between respondents and non-respondents. Therefore a non-response analysis was conducted to determine if those physicians, who chose not to respond to any of the surveys, were in any way different from those physicians who did respond. The non-respondents were mailed a brief, half-page questionnaire to ascertain their reason for not responding to the survey. Several options were presented including: no time, did not receive the survey, forgot, survey misplaced, lost the survey, topic irrelevant, no incentive, don't like to respond to survey, too long, not enough information and not interested. A few critical items from the study survey instrument were also included. These were utilized to compare the demographic and practice characteristics of the non-respondents with the respondents of the study survey, thus determine if there were significant differences between the two groups on key variables that prevent generalization of the study findings. Appendix $\mathrm{G}$ represents the non-response survey. This was also approved by the IRB

\section{$\underline{\text { Data Handling }}$}

Each questionnaire received was checked for completeness. The Statistical Package for Social Sciences ${ }^{\circledR}$ (SPSS) version 10 was used for data entry and statistical analysis. The data was checked for appropriate entry and to assure that data was free of errors. A total of 20 surveys were not included in the analysis as they had more than $50 \%$ 
of the questions incomplete. Thus, the data file contained 261 cases that represent usable responses.

\section{Data Analysis for Phase II}

The data analysis used for this phase is presented by research questions proposed:

Research Question 5: Knowledge of Contraindications and Prescribing Behavior

Descriptive statistics were performed to summarize the correct classification of the different conditions listed in the knowledge section (Section C) of the survey instrument. The absolute contraindications were hypotension, bradycardia, second and third degree AV block. The relative contraindications were systolic blood pressure $<100 \mathrm{Hg}$, heart rate $<60 \mathrm{bpm}$, peripheral vascular disease, peripheral hypoperfusion, ejection fraction $(\mathrm{EF})<40 \%$, first degree AV block, COPD, asthma, insulin dependent diabetes mellitus (IDDM), PR interval on ECG>0.24 second, and betablocker intolerance. The conditions which were neither absolute nor relative contraindication, were hypertension, non-insulin dependent diabetes mellitus (NIDDM), stroke, and ACE inhibitor intolerance. Three scores were calculated based on their correct classification (number of correct responses) - the absolute contraindication score, the relative contraindication score, and the not a contraindication score.

Prescribing rate for beta-blocker therapy was calculated by dividing the number of patients, of the last $10 \mathrm{AMI}$ patients, the respondents prescribed beta-blockers, divided by 10 . This was multiplied by 100 to get the prescribing percent, which was used as a proxy for prescribing behavior. The correlation of this prescribing behavior with the 
three computed scores was estimated to determine the association of prescribing behavior for beta-blockers as a secondary preventive agent after an AMI and knowledge of contraindication of beta-blockers.

\section{Research Question 6: Willingness to Prescribe and Prescribing Behavior}

Descriptive statistics were performed to get the mean of willingness to prescribe, for each of the conditions listed in the Section B of the survey. These were divided into those conditions where beta-blockers should definitely be prescribed, as they definitely provide benefits, conditions where they may be prescribed, as there is uncertainty about their benefits to patients with these conditions, and conditions where they definitely should not be prescribed as they do not provide benefits and can be harmful.

The conditions in the definitely prescribe category were: age greater than 50 years, large or anterior AMI, previous infarction, angioplasty, by-pass surgery, stroke, hypertension, and complex ventricular ectopy. The conditions in the maybe prescribe category were: age less than or equal to 50 years, small infarction, $\mathrm{EF}<40 \%$, history of CHF, systolic blood pressure $<100 \mathrm{Hg}$, heart rate $<60 \mathrm{bpm}, \mathrm{LV}$ failure, first degree AV block, peripheral vascular disease, COPD, asthma, and IDDM. The conditions in the definitely not prescribe category were: hypotension, bradycardia, second and third degree AV block. Three scores were calculated by summations of the answers to the conditions in each of these categories-the definitely prescribe score, the maybe prescribe score and the definitely not prescribe score. The correlation of prescribing behavior calculated in research question 5 with these three computed scores were estimated to determine the 
association of prescribing behavior for beta-blockers as a secondary preventive agent after an AMI and willingness to prescribe.

\section{Research Question 7: Demographic and Practice Characteristics and Prescribing Behavior}

The association of prescribing percent/behavior calculated in research question 5 with demographic and practice characteristics such as age, gender, specialty, year of board certification, location, type of primary practice site, number of beds (size of hospital) and number of patients (both new and repeat) was investigated. Association of prescribing percent with categorical variables such as gender, specialty, location, year of board certification and type of primary practice site were evaluated using t-tests and Ftests. Association of prescribing percent with continuous variables such as age, size of hospital, and number of patients (both new and repeat) was investigated using Pearson's correlations.

Research Question 8: Knowledge of Contraindications, Willingness to Prescribe and Demographic and Practice Characteristics

The association of the three knowledge scores estimated in research question 5 and the three willingness to prescribe scores estimated in research question 6 with demographic and practice characteristics such as age, gender, specialty, year of board certification, location, type of primary practice site, number of beds (size of hospital) and number of patients (both new and repeat) was investigated. Association of each individual score with categorical variables such as gender, specialty, year of board 
certification, location, and type of primary practice site was evaluated using t-tests and Ftests. Association of each individual score with continuous variables such as age, size of hospital, and number of patients (both new and repeat) was investigated using Pearson's correlations.

\section{Research Question 9: Physician Characteristics and Prescribing Behavior}

Association of all physician characteristics with prescribing behavior was investigated using multiple regression analysis. The dependent variable was prescribing percent calculated in research question 5. The independent variables were age, gender, specialty, primary practice site, the three knowledge scores estimated in research question

5 , the three willingness to prescribe scores estimated in research question 6 , and number of AMI patients treated (both new and repeat). Size of hospital, and location were not investigated in this analysis as this question was answered by only those physicians who reported their primary practice site as hospitals. Year of board certification was excluded, as more than $33 \%$ of the respondents did not answer this question and inclusion of this variable would decrease the number of cases in the analysis below the required minimum for adequate power.

\section{Research Question 10: Reasons for Not Prescribing Beta-Blockers}

The reasons for not prescribing beta-blockers in post-AMI patients for secondary prevention given by respondents were combined and enlisted. 
This chapter discussed in detail the methodology employed to fulfill the goals and research questions of both phases I and II. In the next chapter, results of the analysis conducted for accomplishing these will be presented. 


\section{CHAPTER FOUR}

\section{RESULTS AND DISCUSSION}

Earlier chapters of this text provided an overview of the effect of beta-blockers on outcomes, the effect of physician knowledge on prescribing, need for study, and study objectives. The methods employed to achieve these objectives were also outlined. This chapter presents the findings of the study and discussion for the results obtained. Results of phase I are presented first, followed by phase II in the second half of the chapter.

\section{Phase I}

Phase I of the study involved analyzing claims data for those patients who suffered from an acute myocardial infarction (AMI) from January 1, 1997 to June 30, 2000. The purpose was to determine prescribing of beta-blockers as long-term therapy following an AMI and effect of this prescribing on patient outcomes such as mortality, morbidity, health care utilization, and costs.

Paid claims data relevant for the study were obtained from Consultec, Inc. Consultec, Inc. stores data going back to several years. However, not all of this data was pertinent to this study and so relevant data between January 1, 1996 to June 30, 2001 was obtained to conduct the analysis. Table 1 outlines the extraction process from the raw data. A total of 1,940 patients had a hospital claim for AMI and were continuously eligible for one year before the AMI. Of these, 3 patients were excluded as they were part of managed care. The next criterion was age limit. A total of 748 patients were excluded, as they were older than 64 years of age. This also excluded the Medicare 
eligible patients. Those patients who had length of stay less than 3 days (indicating a possible misdiagnosis) and those with ICD-9 (International Classification of Disease $9^{\text {th }}$ edition) codes of 410.x2 were excluded in the next step. This gave a cohort of 599 patients.

In this cohort there were some patients who had a recurrent AMI. In such situations only the first AMI during the selected time frame was included. Additionally, those patients who did not receive beta-blockers in the first 90 days after discharge from the incident AMI but received a prescription after the first 90 days were excluded. The reason being the objective of the study, which was to evaluate the effect of beta-blockers when given long-term after an AMI according to American College of Cardiology (ACC)/American Heart Association (AHA) guidelines. These patients did receive the drug, but this was not according to the guidelines and inclusion of these patients would lead to misclassification bias. A total of 20 patients were excluded because they died during the incident AMI or within 30 days of the incident AMI. Thus, a total of 488 patients satisfied the inclusion criteria and were further investigated for beta-blocker use and its effect on mortality. A total of 18 patients died due to causes not related to AMI. These were excluded from the analysis that investigated effect of appropriate/inappropriate beta-blocker use on cardiac deaths. From this cohort, 58 patients were excluded for investigating beta-blocker use and morbidity and health care utilization, as they were not continuously eligible during the 12-month period after the incident AMI. Thus, 412 patients satisfied all the inclusion criteria for evaluating betablocker use and its effect on morbidity and health care utilization following an AMI. 
Table 1: Cohort Selection

\section{$\begin{array}{lll}\text { Inclusion Criteria } & \text { Patients Excluded } & \text { Selected Cohort }\end{array}$}

First extraction- patients

with AMI and

1,940

continuously eligible for

one year before AMI

Managed care recipients

3

1,937

Age $>64$ years and

1,189

Medicare eligible patients

590

599

Length of Stay $<3$ days

and/or ICD-9 of 410.x 2

91

508

Duplicates and received

beta-blockers after the

initial 90 days of discharge

Death during incident AMI

488 => final cohort for all

or within 30 days of

incident AMI

Death due to non-cardiac

18 cause mortality

causes

death due to cardiac

Continuously eligible for one year after AMI mortality

excluding those who died within the year

412 => final cohort for morbidity and utilization related objectives

AMI: Acute myocardial infarction, ICD-9: International classification of disease $9^{\text {th }}$ edition 


\section{Demographic and Medical History of Patients in Phase I}

The demographic characteristics of the selected cohort is presented in Table 2. The mean age of the cohort at incident AMI was 53.76 (S.D. \pm 8.14) years. More than half $(68.2 \%)$ were over the age of 50 years with majority between the age of 50 to 60 years. About one-third (31.7\%) were below the age of 50, with $22.3 \%$ in the age group of 40 to 50 years. This positive skewness in the age distribution confirms that AMI is an elderly condition and is more prevalent in higher age groups. The study cohort consisted of 246 males (50.4\%), 238 females (48.8\%) and $0.8 \%$ who did not report their gender and were excluded. This is not consistent with the gender distribution of the Medicaid population which is predominantly female (In the year 1999, the distribution of WV Medicaid was 43:56, M:F). This could be due to the fact that AMI is more common among males in this age group (below 65 years) compared to females. The majority of the cohort (91.6\%) was white, $4.1 \%$ were black and the remaining $4.3 \%$ belonged to other ethnic groups. This is characteristic of the West Virginia Medicaid population which consists of a white majority (In the year 1999, 92\% of the Medicaid recipients were white).

Medical history and other characteristics of the selected cohort are represented in Table 3. About $45 \%$ of the patients had hypertension, $37 \%$ were diabetic, and about $7 \%$ had peripheral vascular disease. Approximately $20 \%$ of the patients had suffered from congestive heart failure (CHF) in the year before the incident AMI and 28\% had suffered a previous AMI. A very small percent of patients had a previous coronary artery bypass graft (CABG) $(1.2 \%)$ and none had a previous percutaneous transluminal coronary angioplasty (PTCA). Around 6\% of the patients had suffered a stroke, and less than 1 
percent had renal dysfunction. Over $11 \%$ had some absolute contraindications and over $60 \%$ had some relative contraindications for beta-blocker use following an AMI. The mean number of cardiac admissions and non-cardiac admissions in the previous year was less than 1. However, the range was broader for non-cardiac admissions (0-5). The mean number of different prescription drugs the patients were on was 10.49. Less than onethird of the patients were on medications associated with AMI such as calcium channel blockers (31.6\%), Angiotensin converting enzyme (ACE) inhibitors (29.1\%), betablockers $(22.7 \%)$, digoxin $(12.3 \%)$, and anti-arrhythmics $(2.5 \%)$, and in the six months prior to the incident AMI. A very small number of the patients underwent a CABG (10\%) or PTCA (about 16\%) during the incident AMI. The mean length of stay during the incident AMI was 7 and the patients had an average of 4 secondary diagnoses at index AMI. Among the discharge medications, about $64 \%$ were prescribed beta-blockers, $40 \%$ were prescribed ACE-inhibitors, $38 \%$ were prescribed loop diuretics, $27 \%$ were prescribed calcium channel blockers, and 12\% were prescribed aspirin. 
Table 2: Demographic Characteristics of the Final Phase I Study Patients

$\begin{array}{lll}\text { Demographic } & \text { N } & \text { Percent } \\ \text { Characteristics } & \end{array}$

Age

$\begin{array}{lcc}\leq 30 \text { years } & 4 & 0.8 \\ 30<\text { to } \leq 40 \text { years } & 42 & 8.6 \\ 40<\text { to } \leq 50 \text { years } & 109 & 22.3 \\ 50<\text { to } \leq 60 \text { years } & 212 & 43.5 \\ 60<\text { to } \leq 64 \text { years } & 121 & 24.8 \\ \text { Total } & 488 & 100.0\end{array}$

Average Age \pm S.D. $\quad 53.76 \pm 8.14$

Gender

$\begin{array}{ccc}\text { Males } & 246 & 50.4 \\ \text { Females } & 238 & 48.8 \\ \text { Unknown } & 4 & 0.8 \\ \text { Total } & 488 & 100.0\end{array}$

Race

$\begin{array}{lcc}\text { White } & 447 & 91.6 \\ \text { Black } & 20 & 4.1 \\ \text { Others } & 21 & 4.3 \\ \text { Total } & 488 & 100.0\end{array}$

N: Number of patients, S.D: Standard deviation 
Table 3: Medical History and Other Characteristics

Characteristics*

Hypertension

Diabetes

Previous AMI

Previous CHF

Peripheral vascular disease

Previous stroke

Previous CABG

Renal dysfunction

Previous PTCA

Presence of relative contraindications

Presence of absolute contraindications

Pre-AMI medications received

Calcium channel blockers

ACE inhibitors

Beta-blockers

Digoxin

Anti-arhythymics
$\mathbf{N}$

221

179

138

97

33

30

6

4

0

295

55

154

142

111

60

12

Procedures during the incident AMI

PTCA

15.8
11.3

2.5

Percent

45.3

36.7

28.3

19.9

6.8

6.1

1.2

0.8

0.0

60.5

31.6

29.1

22.7

12.3 
Table 3: Medical History and Other Characteristics (contd.)

\begin{tabular}{lcc}
\hline Characteristics* & N & Percent \\
\hline CABG & 50 & 10.2 \\
Discharge Medications & 310 & 63.5 \\
$\quad$ Beta-blockers & 193 & 39.5 \\
$\quad$ ACE-inhibitors & 183 & 37.5 \\
$\quad$ Loop diuretics & 132 & 27.0 \\
$\quad$ Calcium channel blockers & 58 & 11.9 \\
$\quad$ Aspirin & Mean (S.D.) & Min-Max \\
$\quad$ & $0.26(0.44)$ & $0-1$ \\
$\quad 0.30(0.68)$ & $0-5$ \\
Number of cardiac admissions in the prior year & $10.49(7.18)$ & $0-42$ \\
Number of non-cardiac admissions in the prior year & $7.36(4.49)$ & $4-38$ \\
Number of different drugs in the prior six months & $4.17(2.88)$ & $1-9$ \\
Length of stay during incident AMI & & \\
Number of secondary diagnosis at index AMI & & \\
\hline
\end{tabular}

N: Number of patients, AMI: Acute myocardial infarction, CHF: Congestive heart failure, CABG: Coronary artery bypass graft surgery, PTCA: Percutaneous transluminal coronary angioplasty, ACE: Angiotensin converting enzyme S.D: Standard deviation, Min: Minimum value, Max: Maximum value * Medical history and presence of various conditions were determined using International classification of disease- $9^{\text {th }}$ edition (ICD-9) codes, Common procedural terminology (CPT) codes and Diagnosis related group (DRG) codes. Medication use was determined by using generic and National drug classification (NDC) codes 


\section{Results for Research Question 1}

The first research question of the study was to determine prescribing patterns of betablocker therapy for secondary prevention following an AMI and to see whether it was consistent with recommendations by AHA/ACC.

A total of $310(63.5 \%)$ patients received beta-blocker prescription within 90 days of discharge. When beta-blocker use was determined at the end of 30 days and 60 days from discharge, the number of patients was lower with $282(57.8 \%)$ receiving betablocker prescription at both occasions. For study purposes, beta-blocker use was defined as a prescription of beta-blocker within 90 days of discharge from the incident AMI. This allowed sufficient time to pass, after discharge, in case the patients received an initial supply of medications with refill orders from the hospital during discharge for which there would be no prescription record. Based on the presence or absence of absolute contraindications to beta-blocker and presence of beta-blocker usage, the patients were classified to those who received beta-blocker appropriately and those who received beta-blocker inappropriately (See Figure 6). Thus, those who had the absolute contraindications and received therapy were classified in the misuse category, and those who did not have absolute contraindications and did not received therapy were classified in the underuse category. Both misuse $(5.7 \%)$ and underuse $(30.9 \%)$ formed the inappropriately prescribed category (36.7\%). Similarly, those patients who did not have absolute contraindications and received therapy (57.8\%) and those who did have absolute contraindications and did not receive therapy (5.5\%) were classified as appropriately prescribed (63.3\%) (See Table 4). 
Univariate Analysis was conducted to see if the groups (appropriately prescribed and inappropriately prescribed) were different from each other (Table 5). The two groups were compared on the following variables: Age, gender, presence of diabetes, presence of hypertension, presence of peripheral vascular disease, presence of renal dysfunction, presence of previous AMI, $\mathrm{CHF}$, stroke, previous $\mathrm{CABG}$, presence of relative and absolute contraindications, number of cardiac and non-cardiac admissions in the preperiod, number of drugs in the 6 months before AMI, procedures during incident AMI (CABG, PTCA), length of stay during incident AMI, number of secondary diagnosis at incident AMI and pre-AMI medication use (ACE inhibitors, anti-arrhythmic agents, betablockers, calcium channel blockers \& digoxin,). T-tests were done for continuous variables and chi-square analysis was performed for categorical variables. Among these different variables, age, presence of hypertension, presence of absolute contradindications, number of non-cardiac admissions in the pre period, and use of digoxin, and beta-blockers in the pre-period were significantly different between the two groups. The appropriate group was slightly younger, had more hypertensive patients, had fewer patients with absolute contraindications, and fewer patients who had fewer noncardiac admissions in the pre-period as compared to the inappropriate group. Additionally, the appropriate group had more patients who received prescriptions of betablockers but fewer patients who received prescriptions for digoxin in the pre-period compared to the inappropriate group. Though not significant the appropriate group had higher percentage of patients with stroke and previous CABG compared to the inappropriate group. 
A logistic regression analysis was conducted to predict assignment of patients to the appropriate and inappropriate group. Those variables that were significant in the univariate analysis were used as independent variables. Thus, the independent variables included were age, presence of hypertension, presence of absolute contraindications, number of non-cardiac admissions in the pre-period, use of digoxin, and beta-blockers in the pre-period. Table 6 represents results of this analysis. The model was significant $\left(\chi^{2}\right.$ $=58.003, \mathrm{p}=0.000, \mathrm{n}=488)$ with an $\mathrm{R}^{2}$ of $15.3 \%$ and $67.42 \%$ correct classification. Five of the six independent variables were significant predictors of group status. These were age, hypertension, presence of absolute contraindications, number of non-cardiac admissions in the pre-period, and use of beta-blockers in the pre-period. Those who were hypertensive were almost twice as likely to belong to the appropriate group, whereas those who received beta-blockers in the pre-period were 2.6 times more likely to belong to the appropriate group. Older patients, those with absolute contraindications and those with more non-cardiac admissions in the pre-period were more likely to belong to the inappropriate group. The predicted value of this regression or propensity score, was utilized in future analysis as a co-variate to reduce bias due to the differences between the two groups. 
Figure 6: Appropriate/Inappropriate Use of Beta-blockers

Presence of Absolute Contraindications

\begin{tabular}{|c|c|c|}
\hline & Yes & No \\
\hline $\begin{array}{l}\text { Beta-blocker } \\
\text { Use }\end{array}$ & Misuse & Appropriate use I \\
\hline No & Appropriate use II & Under use \\
\hline
\end{tabular}


Table 4: Prescription Patterns for Beta-blocker Use

\begin{tabular}{lcc}
\hline Category & N & Percent \\
\hline Appropriate use & 309 & 63.3 \\
Appropriate use I & 282 & 57.8 \\
Appropriate use II & 27 & 5.5 \\
$\quad 179$ & 36.7 \\
Inappropriate Use & 151 & 30.9 \\
Under use & 28 & 5.7 \\
Misuse & & 100.0 \\
Total & 488 & \\
\hline
\end{tabular}

$\mathrm{N}$ : Number of patients 
Table 5: Univariate Comparisons between the Two Groups

\begin{tabular}{|c|c|c|c|c|}
\hline Variables & $\begin{array}{l}\text { Appropriate } \\
\text { Group \% }\end{array}$ & $\begin{array}{l}\text { Inappropriate } \\
\text { Group \% }\end{array}$ & $\begin{array}{l}\text { Test } \\
\text { Statistic }\end{array}$ & $\begin{array}{l}\text { Significance } \\
\text { (p) }\end{array}$ \\
\hline Age Mean (S.D.) & $52.72(8.58)$ & $55.56(7.00)$ & $\mathrm{t}=3.975$ & $0.000 *$ \\
\hline Gender & & & $\chi^{2}=0.215$ & 0.643 \\
\hline Males & 51.6 & 49.4 & & \\
\hline Females & 48.4 & 50.6 & & \\
\hline Diabetes & 35.3 & 39.1 & $\chi^{2}=0.716$ & 0.397 \\
\hline Hypertension & 50.5 & 36.3 & $\chi^{2}=9.188$ & $0.002^{*}$ \\
\hline $\begin{array}{l}\text { Peripheral vascular } \\
\text { disease }\end{array}$ & 6.8 & 6.7 & $\chi^{2}=0.002$ & 0.969 \\
\hline Renal dysfunction & 1.0 & 0.6 & $\chi^{2}=0.237$ & 0.626 \\
\hline Previous AMI & 29.1 & 26.8 & $\chi^{2}=0.298$ & 0.585 \\
\hline Previous CHF & 19.1 & 21.2 & $\chi^{2}=0.324$ & 0.569 \\
\hline Previous Stroke & 7.1 & 4.5 & $\chi^{2}=1.380$ & 0.240 \\
\hline Previous CABG & 1.6 & 0.6 & $\chi^{2}=1.048$ & 0.306 \\
\hline $\begin{array}{l}\text { Presence of relative } \\
\text { contraindications }\end{array}$ & 59.2 & 62.6 & $\chi^{2}=0.531$ & 0.466 \\
\hline $\begin{array}{l}\text { Presence of absolute } \\
\text { contraindications }\end{array}$ & 8.7 & 15.6 & $\chi^{2}=5.403$ & $0.020^{*}$ \\
\hline $\begin{array}{l}\text { Number of cardiac } \\
\text { admissions Mean } \\
\text { (S.D.) }\end{array}$ & $0.28(0.45)$ & $0.23(0.42)$ & $\mathrm{t}=1.215$ & 0.225 \\
\hline $\begin{array}{l}\text { Number of non- } \\
\text { cardiac admissions } \\
\text { Mean (S.D.) }\end{array}$ & $0.24(0.61)$ & $0.40(0.78)$ & $\mathrm{t}=2.454$ & $0.015^{*}$ \\
\hline
\end{tabular}


Table 5: Univariate Comparisons between the Two Groups (contd.)

\begin{tabular}{|c|c|c|c|c|}
\hline Variables & $\begin{array}{l}\text { Appropriate } \\
\text { Group \% }\end{array}$ & $\begin{array}{l}\text { Inappropriate } \\
\text { Group \% }\end{array}$ & $\begin{array}{l}\text { Test } \\
\text { Statistic }\end{array}$ & $\begin{array}{l}\text { Significance } \\
\text { (p) }\end{array}$ \\
\hline $\begin{array}{l}\text { Number of different } \\
\text { drugs in the prior six } \\
\text { months Mean (S.D.) }\end{array}$ & $10.66(7.51)$ & $10.20(6.56)$ & $t=0.694$ & 0.488 \\
\hline $\mathrm{CABG}$ & 10.0 & 10.6 & $\chi^{2}=0.046$ & 0.838 \\
\hline PTCA & 17.2 & 13.4 & $\chi^{2}=1.196$ & 0.274 \\
\hline $\begin{array}{l}\text { Length of stay Mean } \\
\text { (S.D.) }\end{array}$ & $7.20(4.63)$ & $7.65(4.23)$ & $\mathrm{t}=1.083$ & 0.280 \\
\hline $\begin{array}{l}\text { Number of secondary } \\
\text { diagnosis Mean } \\
\text { (S.D.) }\end{array}$ & $4.30(2.89)$ & $3.95(2.85)$ & $\mathrm{t}=1.299$ & 0.194 \\
\hline \multicolumn{5}{|l|}{$\begin{array}{l}\text { Pre-AMI medications } \\
\text { received: }\end{array}$} \\
\hline ACE inhibitors & 28.2 & 30.7 & $\chi^{2}=0.363$ & 0.547 \\
\hline Anti-arhythymics & 2.9 & 1.7 & $\chi^{2}=0.723$ & 0.395 \\
\hline Beta-blockers & 28.8 & 12.3 & $\chi^{2}=17.586$ & $0.000 *$ \\
\hline $\begin{array}{l}\text { Calcium channel } \\
\text { blockers }\end{array}$ & 31.7 & 31.3 & $\chi^{2}=0.010$ & 0.921 \\
\hline Digoxin & 10.0 & 16.2 & $\chi^{2}=4.000$ & $0.046 *$ \\
\hline
\end{tabular}

* Significant at $\mathrm{p} \leq 0.05$, S.D: Standard deviation, AMI: Acute myocardial infarction, CHF: Congestive heart failure, $\mathrm{CABG}$ : Coronary artery bypass graft, PTCA: Percutaneous transluminal coronary angioplasty, ACE: Angiotensin converting enzyme 
Table 6: Predictors for Appropriate/Inappropriate Group

\begin{tabular}{|c|c|c|c|c|}
\hline Predictor & Estimate & S.E. & Odds Ratio & Significance \\
\hline Age & -0.0529 & 0.0135 & 0.9484 & $0.000 *$ \\
\hline Hypertension & 0.6420 & 0.2074 & 1.9002 & $0.002 *$ \\
\hline $\begin{array}{l}\text { Presence of absolute } \\
\text { contraindications }\end{array}$ & -0.7914 & 0.3056 & 0.4532 & $0.009 *$ \\
\hline $\begin{array}{l}\text { Number of non-cardiac } \\
\text { admissions }\end{array}$ & -0.4317 & 0.1478 & 0.6494 & $0.003 *$ \\
\hline \multicolumn{5}{|l|}{ Pre-AMI medications: } \\
\hline Digoxin & -0.3000 & 0.3010 & 0.7408 & 0.318 \\
\hline Beta-blockers & 0.9916 & 0.2723 & 2.6956 & $0.003 *$ \\
\hline \multicolumn{5}{|c|}{$\mathrm{R}^{2}=15.3 \%\left(\chi^{2}=58.003, \mathrm{p}=0.000\right), \mathrm{n}=488$} \\
\hline
\end{tabular}

* Significant at $\mathrm{p} \leq 0.05$, S.E: Standard error, AMI: Acute myocardial infarction 


\section{Discussion for Research Question 1}

An analysis of the data revealed that there was inappropriate utilization of betablockers as secondary preventive agents following an AMI. Compared to previous studies where the utilization among eligible patients ranged from $30-40 \%$, this study revealed a utilization of almost 64\% (Rogers et al., 2000; Krumholz et al., 1998 \& Soumerai et al., 1997). This was also higher compared to the utilization levels reported recently in a Medicare population in the state of West Virginia, the same state as the study state, where only 59.4\% received therapy at discharge (Schade et al., 2002). Thus, there appears to be an increase in utilization of beta-blockers among eligible patients over time. This confirms previous reports of steady rise in average use over years reported by Heller et al., (39.6\% to 58.6\%, 2000) and Pilote et al., (33\% to 50\%, 2000). Thus, physicians are responding to the increase in awareness about the beneficial effects of beta-blockers for AMI patients. The results also demonstrated a 5.7\% use among patients with absolute contraindications. This proportion is smaller than that reported in previous studies, where it was $11 \%$ (Brand et al., 1995) and 24\% (Sial et al., 1994). A subanalysis was conducted to investigate the distribution of age in the misuse, under use and appropriate use categories. This study confirmed the association of age with beta-blocker use as reported in previous studies (Fishkind et al., 1997 ;Gurwitz et al., 1992). Even in this younger population, the under use group was older (56.1 years) than the appropriate use (52.7 years) and misuse groups (52.7 years) and this was statistically significant $(\mathrm{F}=$ $9.29, \mathrm{p}<0.0001)$. Thus, though this study revealed an increase in use of beta-blocker therapy in post AMI patients, there is still room for improvement in patients in whom it would maximize benefits. 
A comparison of the two groups classified for study purposes revealed that they were different from each other on a few variables. However, this could not help identify which group was more severely ill between the appropriate and inappropriate. A multivariate comparison revealed that five of these variables were significant predictors of group status. These include- age, hypertension, presence of absolute contraindications, number of non-cardiac admission in pre-period, and use of beta-blockers in the preperiod. Patients who were older, had presence of absolute contraindications, and had a greater number of non-cardiac admissions were in the inappropriate group, whereas patients with hypertension and pre-beta-blocker use were in the appropriate group.

\section{Results for Research Question 2}

Kaplan-Meier survival curves were constructed to investigate effect of appropriate and inappropriate prescribing of beta-blocker therapy on mortality for the selected patients in the first-year after the incident AMI. Two separate analyses were conducted. The first included death due to all causes and the second included death due to cardiac causes. Figure 7 illustrates the all-cause death curves and Figure 8 illustrates the cardiac-cause death curves. The two groups (appropriate and inappropriate) differed significantly for survival at the end of the one-year period $(\log \operatorname{Rank}$ Statistic $=4.44, \mathrm{p}=$ 0.0351). The appropriate group had a better survival distribution compared to the inappropriate group. An investigation of only cardiac deaths revealed better survival distribution for the appropriate group too (Figure 8), however this was not statistically significant (Log Rank Statistic $=1.35, \mathrm{p}=0.245)$. 
The different causes of deaths are displayed in Table 7. The non-cardiac deaths were not related to AMI and were mostly due to cancer or diabetes. A majority of the cardiac deaths were due to coronary artery disease $(\mathrm{CAD})$ and acute myocardial infarction (AMI). A frequency of cardiac deaths by group is represented in Table 8. This shows that there were more deaths due to AMI in the inappropriate group. Power analysis revealed that time to all cause death analysis had a power of $88 \%$ whereas time to cardiac death had a power of $46 \%$. The numbers of deaths in the two groups were investigated by chi-square analysis. The results were similar to the survival analysis, with the difference between the two groups being significant for all cause deaths and not significant for cardiac-cause deaths (Table 9). Additionally, the groups were compared for deaths due to an AMI. Even though the percentage was higher in the inappropriate group this was not statistically significant. When analysis was repeated using betablocker prescribing at 30 and 60 days after the AMI to categorize appropriate and inappropriate prescribing, the results for both all-cause and cardiac mortality did not change. 
Figure 7: Kaplan-Meier Survival Curves (all-cause deaths) for Appropriate/Inappropriate Use of Beta-blockers

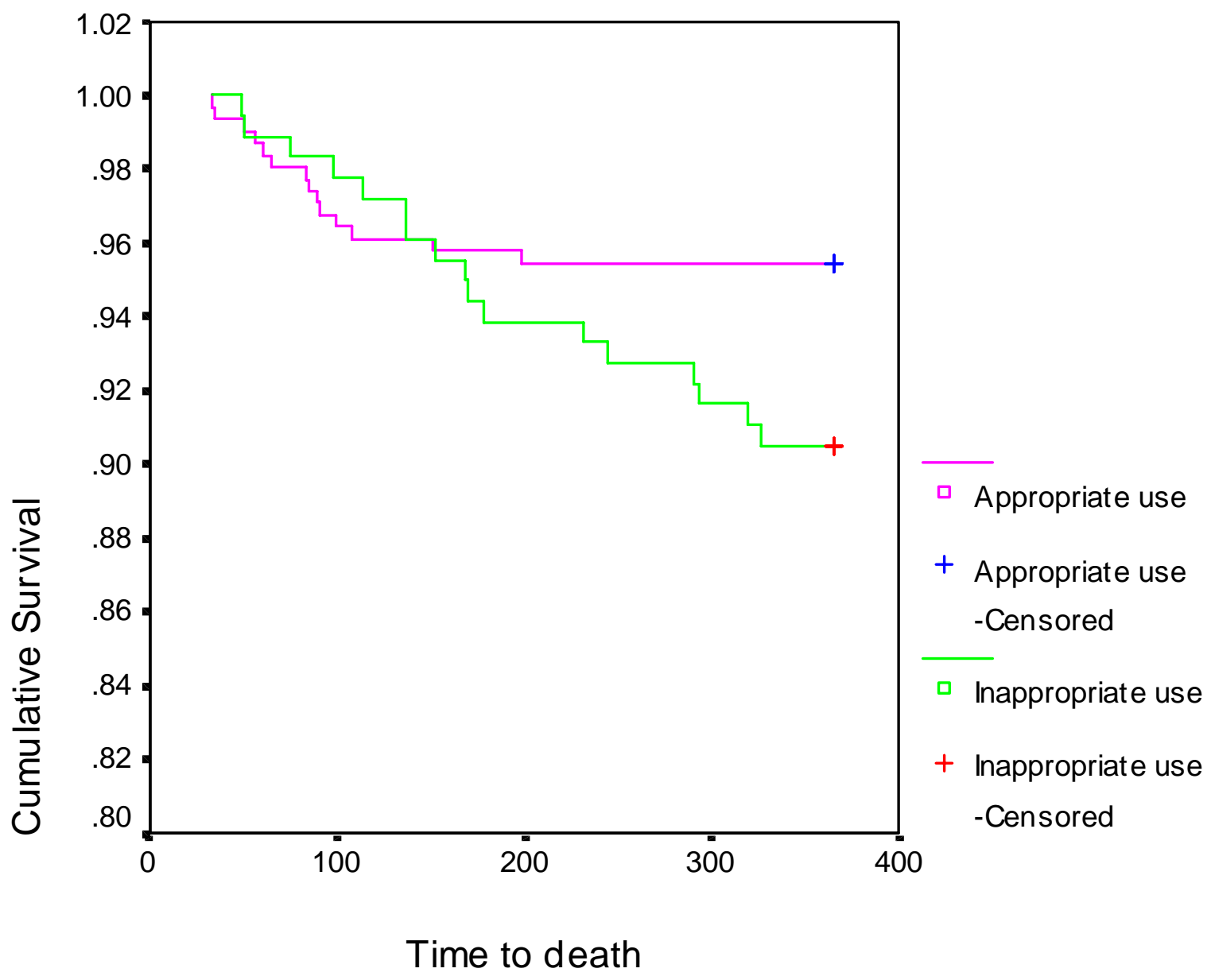


Figure 8: Kaplan-Meier Survival Curves (cardiac deaths) for Appropriate/Inappropriate Use of Beta-blockers

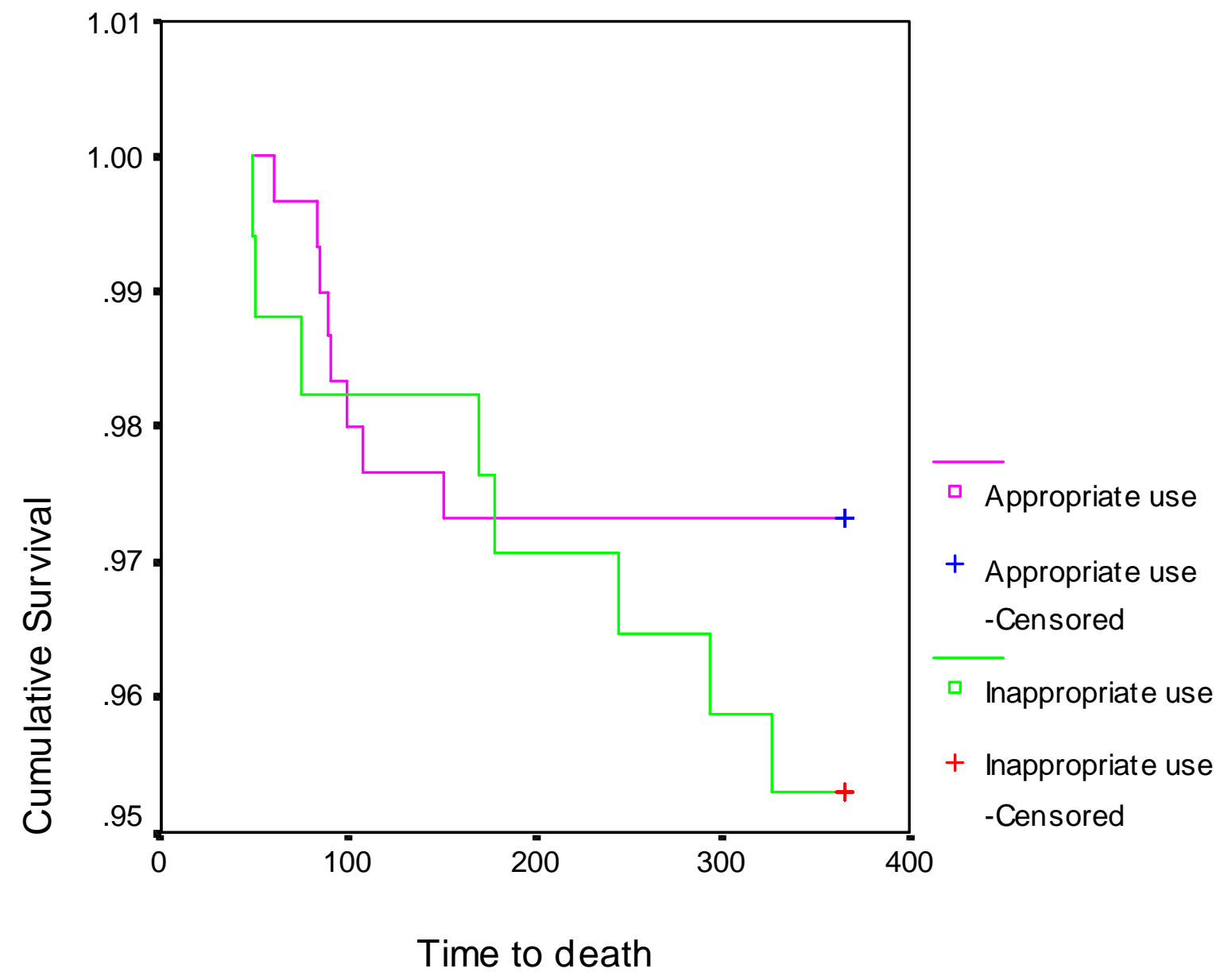


Table 7: Causes of Death

Non-cardiac Causes $(n=15) \quad$ Cardiac Causes $(n=16)$

Accident (1)

Coronary arteriosclerosis (5)

AIDS (1)

Dysrhythmia (1)

Cancer of trachea, lung, and bronchi (4)

Heart disease, unspecified (1)

Chronic obstructive pulmonary disease (1)

Ischemia (1)

Diabetes (4)

Acute myocardial infarction (8)

End stage renal disease (1)

Injury to peripheral nerves (1)

Kidney disorder (1)

Pulmonary embolism (1) 
Table 8: Cardiac Deaths by Group

Appropriate Use (8) Inappropriate Use (8)

Coronary arteriosclerosis (3) Coronary arteriosclerosis (2)

Dysrhythmia (1) Heart disease (1)

Ischemia (1) Acute myocardial infarction (5)

Acute myocardial infarction (3) 
Table 9: Patient Deaths by Group Status

\begin{tabular}{lccc}
\hline Mortality & Percent & $\chi^{2}$ Statistic & Significance \\
\hline All Cause Death & 4.5 & 4.699 & $0.030^{*}$ \\
Appropriate use & 9.5 & & \\
Inappropriate use & & 1.370 & 0.241 \\
Cardiac Death & & & \\
Appropriate use & 2.7 & & \\
Inappropriate use & 4.7 & & \\
& & & \\
Death due to AMI & 1.0 & & \\
Appropriate use & 2.4 & \\
Inappropriate use & & \\
\hline
\end{tabular}

* Significant at $\mathrm{p} \leq 0.05$, AMI: Acute myocardial infarction 


\section{Discussion for Research Question 2}

There was a significant relationship between beta-blocker receipt among eligible patients for secondary prevention after an AMI and all cause mortality. Thus, null hypothesis A is rejected. The appropriate group had a significantly better distribution of survival in the year after the AMI. This is consistent with the information associated with both clinical trials and observational studies in this area (Soumerai et al., 1997; Bradford et al., 1999). However, beta-blockers have been reported to reduce cardiac mortality by reduction in re-infarction rates and sudden death (Goldsetin, 1996). The chi-square test that investigated this relationship between cardiac mortality and beta-blocker use was not significant. Though the numbers were in favor of appropriate use of beta-blocker therapy, statistical significance was not observed. Thus, the null hypothesis B is not rejected. There could be two reasons for this observation. First, the number of patients in this cohort is small, thus the analysis did not have sufficient power to achieve statistical significance compared to previous studies that involved large numbers of patients. Soumerai et al., (1997) had a cohort of almost 4,000 patients and Krumholz et al., (1998) involved greater than 45,000 patients. The second more important reason is the fact that this study was conducted in a relatively younger population, in which the average age was less than 54 years compared to previous retrospective studies. Thus, intuitively, the probability of death in a younger patient due to AMI would be smaller compared to an older patient greater than 65 years old, and thus this population probably had lower death rates, which did not achieve significance. However, even in this younger population, appropriate use of beta-blocker had a lower proportion of cardiac deaths, compared to the inappropriate group. 
An important fact to consider is that some of the deaths that were excluded when cardiac mortality was investigated were associated with diabetes. Diabetes can be an underlying cause for death but not the actual cause of death, unless it is type I diabetes where acute coma is the leading cause of death (NIH, 1995). The majority of deaths in type II diabetics is due to heart disease (NIH, 1995). Additionally, one of the excluded death was due to an accident. A patient with a previous AMI is more likely to die of an AMI during an accident compared to other causes. Also, another death excluded was due to pulmonary embolism. Pulmonary embolism is one of the complications associated with an AMI (Ahdout, Damani, \& Ultan, 1989). However, a pulmonary embolism can be due to other causes too (Carson et al., 1992). Hence, this death had to be excluded. The information obtained from vital statistics for death is for record purposes and not research purposes. More detailed information about death would probably be helpful in getting the true cause of death. Thus, this identifies using death information from Vital Statistics as a limitation for determining true cause of death.

\section{Results for Research Question 3}

Morbidity between the two groups was compared via Kaplan-Meier survival curves too. However, the dependent variable was time to first hospitalization due to a cardiovascular event from the date of discharge. Here cardiovascular event was defined as first hospitalization due to various conditions related to AMI (See Table 10). This revealed a statistical significant difference in the distribution for the dependent variable by group. The inappropriate group had a better distribution with fewer events compared to the appropriate group (Long rank statistic $=5.42, \mathrm{p}=0.0199$, Figure 9). This analysis 
had a power of $75.5 \%$. When the number of patients was compared, this was also statistically significant (Table 11). However, when similar analysis was repeated for recurrent AMI, both distribution and number were not significant (Figure 10, Table 11).

The significant survival analysis of time to event was followed with Coxproportional hazard's regression model to adjust for patient conditions in the form of covariates. Two models were run, the first one used those variables which were significant predictors of group status (appropriate/inappropriate) as co-variates in addition to some of the identified variables associated with the incident AMI and post-AMI care. The second model used the propensity score calculated as the predicted probability for group status in analysis of research question 1 and the identified variables associated with the incident AMI and post-AMI care.

The first model revealed that survival distribution was significant by group status even after adjusting for the various co-variates. The hazards ratio (HR) for the group was 2.00 , implying that the hazard for the appropriate group was almost two times the hazard for the inappropriate group to suffer from the defined cardiovascular event (Table 12). Some of the other co-variates which were significant in this model were number of noncardiac admissions in the pre-period $(\mathrm{HR}=1.44)$, use of beta-blockers in the pre-period $(\mathrm{HR}=1.47), \mathrm{CABG}$ during incident $\mathrm{AMI}(\mathrm{HR}=0.39)$, and length of stay during incident AMI $(\mathrm{HR}=1.04)$. Days supply of beta-blockers in the follow-up period was almost significant $(\mathrm{HR}=0.99, \mathrm{p}=0.06)$. Thus, those with greater length of stay during incident AMI, pre-period beta-blocker use, and greater number of hospitalization in the pre-period were more likely to suffer from a cardiac event, whereas those who underwent CABG during incident AMI were less likely to suffer from a cardiac event. Also, days supply 
though not statistically significant was associated with lower morbidity. The second model also revealed that survival distribution was significant by group (Table 13). Here the HR for group was 1.98 , and two other variables CABG during incident AMI and length of stay during incident AMI were significant. Thus, results did not change much using propensity score analysis. When analysis was repeated using beta-blocker prescribing at 30 and 60 days after the AMI to categorize appropriate and inappropriate prescribing, the results for morbidity did not change. 
Table 10: Cardiovascular Events for Morbidity

\section{Conditions for Morbidity}

Angina

Cardiogenic shock

Cardiomyopathy

Conduction disorders

Disease of endocardium

Essential hypertension

Heart failure

Ischemia

Ischemic heart disease

Acute myocardial infarction

Stroke 
Figure 9: Kaplan-Meier Survival Morbidity Curves (time to first cardiovascular hospitalization) for Appropriate/Inappropriate Use of Beta-blockers

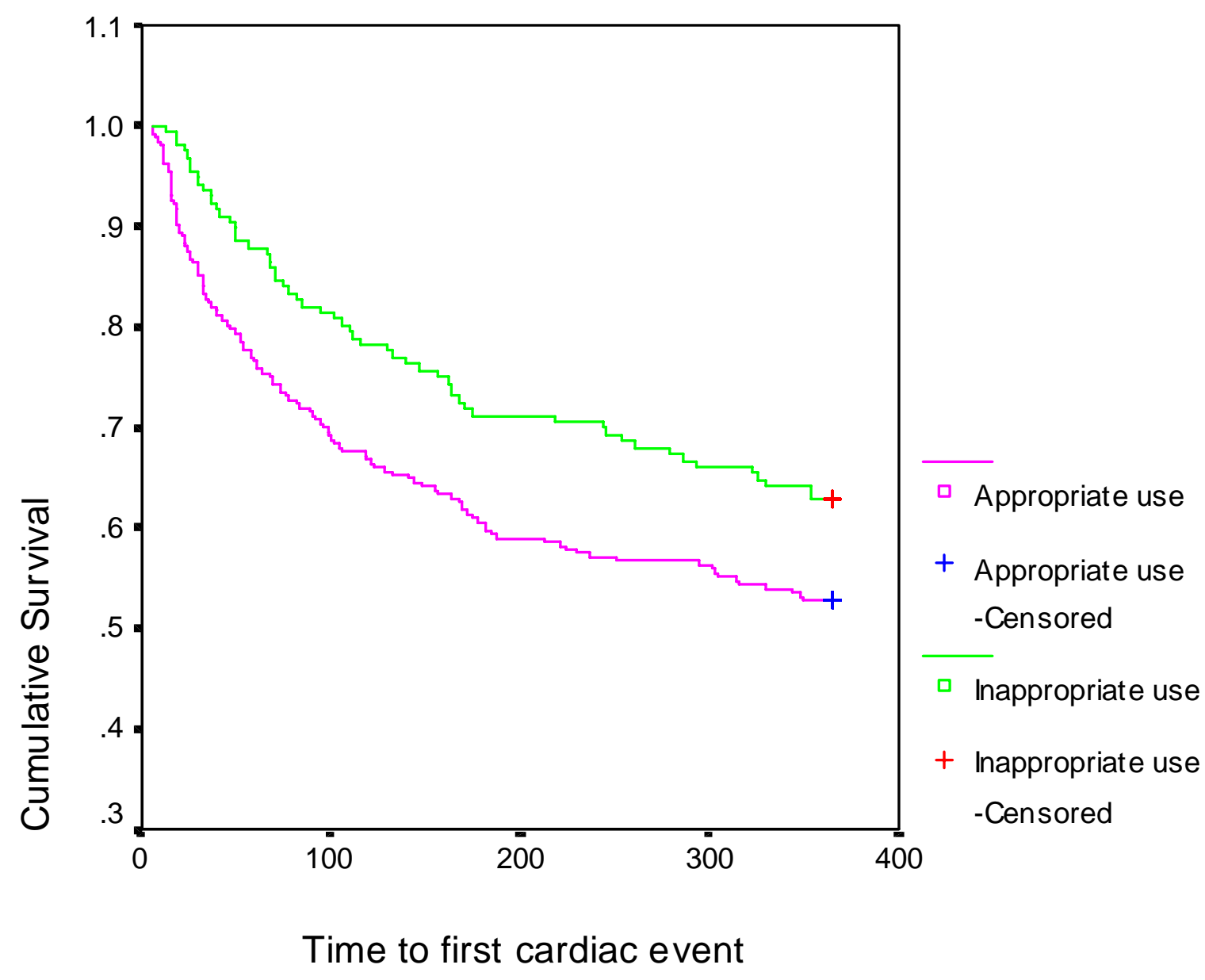


Figure 10: Kaplan-Meier Survival Morbidity Curves (time to recurrent AMI) for Appropriate/Inappropriate Use of Beta-blockers

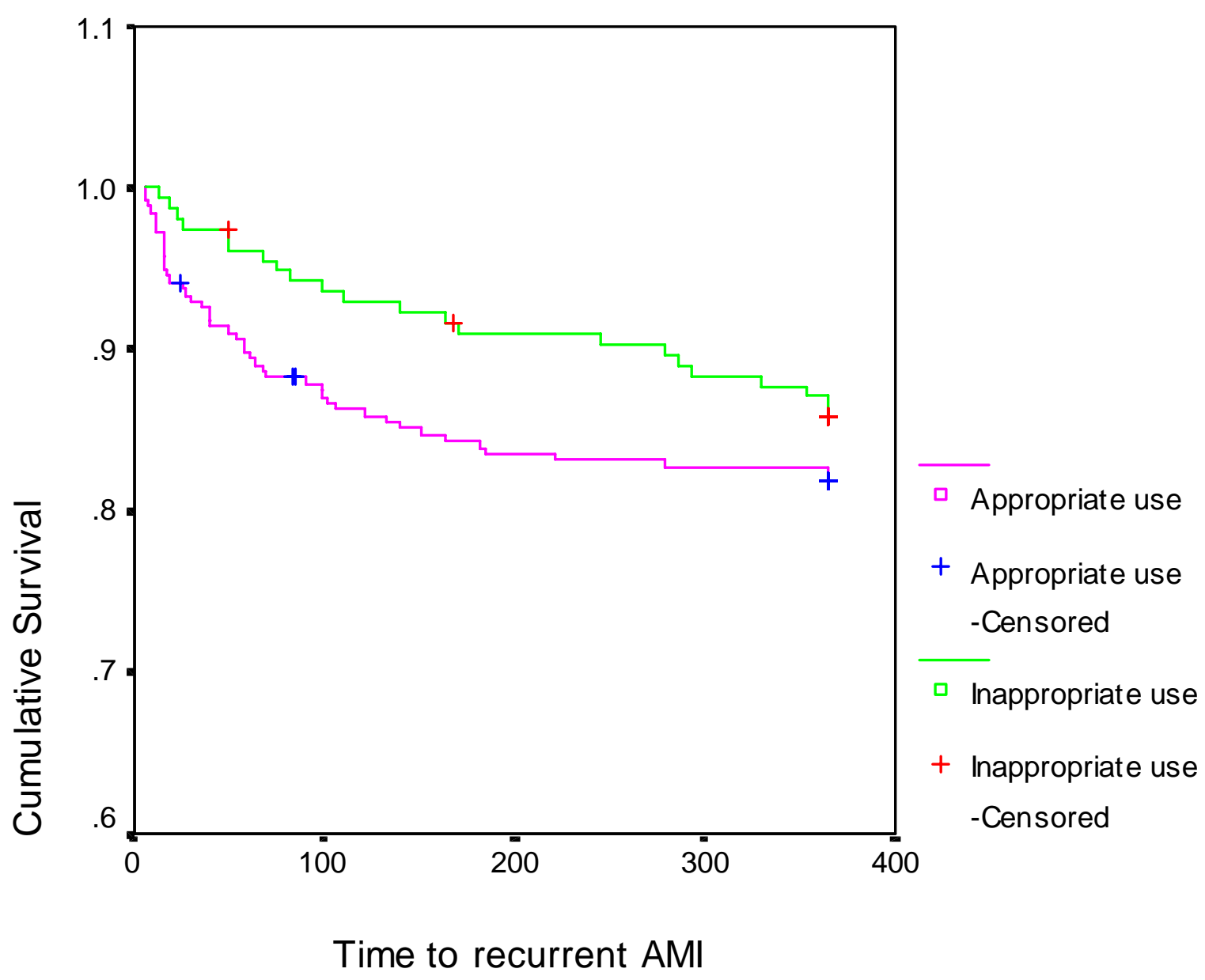


Table 11: Cardiac Events by Group Status

\begin{tabular}{lccc}
\hline Events & Percent & $\chi^{2}$ Statistic & Significance \\
\hline All Cardiac Events & & 4.013 & $0.045^{*}$ \\
Appropriate use & 47.3 & & \\
Inappropriate use & 37.2 & & \\
& & & \\
Recurrent Myocardial Infarction & & & \\
Appropriate use & 18.4 & & \\
Inappropriate use & 14.1 & & \\
\end{tabular}

* Significant at $\mathrm{p} \leq 0.05$ 
Table 12: Cox-Proportional Regression Model for Time to First Cardiac Event

Predictor

Estimate S.E. Hazard's Ratio Significance

\begin{tabular}{lllll}
\hline Age & 0.0076 & 0.0105 & 1.0079 & 0.449 \\
Sex & 0.0857 & 0.1621 & 1.0895 & 0.597 \\
$\begin{array}{l}\text { Appropriate/Inappropriate use } \\
\text { of Beta-blockers at post-AMI }\end{array}$ & 0.6931 & 0.2454 & 2.000 & $0.004^{*}$ \\
$\begin{array}{l}\text { Hypertension } \\
\begin{array}{l}\text { Presence of absolute } \\
\text { contraindications }\end{array}\end{array}$ & -0.1201 & 0.1658 & 0.8869 & 0.469 \\
$\begin{array}{l}\text { Number of non-cardiac } \\
\text { admissions }\end{array}$ & 0.3675 & 0.0979 & 1.4441 & $0.000^{*}$ \\
$\begin{array}{l}\text { Pre-AMI medications: } \\
\quad \text { Beta-blockers }\end{array}$ & & & & \\
$\begin{array}{l}\text { Procedures during the } \\
\text { incident AMI }\end{array}$ & 0.3856 & 0.1927 & 1.4705 & $0.045^{*}$ \\
$\quad$ & & & & \\
$\quad$ CABG & -0.9253 & 0.3275 & 0.3964 & $0.004^{*}$ \\
$\quad$ PTCA & -0.2361 & 0.2293 & 0.7897 & 0.303 \\
$\quad$ Length of stay & 0.0362 & 0.0171 & 1.0369 & $0.034^{*}$
\end{tabular}

Discharge Medications

$\begin{array}{lllll}\text { Aspirin } & 0.0716 & 0.2397 & 1.0742 & 0.765 \\ \text { ACE-inhibitors } & 0.1498 & 0.1614 & 1.1617 & 0.353 \\ \begin{array}{l}\text { Calcium channel } \\ \text { blockers }\end{array} & 0.0639 & 0.1684 & 1.0660 & 0.704 \\ \begin{array}{l}\text { Loop diuretics } \\ \text { cation Possession Ratio }\end{array} & 0.1531 & 0.1695 & 1.1654 & 0.366 \\ & 0.0252 & 0.3099 & 1.0256 & 0.935\end{array}$


Table 12: Cox-Proportional Regression Model for Time to First Cardiac Event (contd.)

\section{Predictor Estimate S.E. Hazard's Ratio Significance}

$\begin{array}{lllll}\text { Day supply } & -0.0014 & 0.0007 & 0.9987 & 0.061^{+}\end{array}$

$$
-2 \mathrm{LL}=1989.8\left(\chi^{2}=47.89, \mathrm{p}=0.000^{*}\right), \mathrm{n}=408
$$

* Significant at $\mathrm{p} \leq 0.05,{ }^{+}$Significant at $\mathrm{p} \leq 0.01$, S.E: Standard error, AMI: Acute myocardial infarction, CABG: Coronary artery bypass graft, PTCA: Percutaneous transluminal coronary angioplasty, ACE:

Angiotensin converting enzyme inhibitor 
Table 13: Cox-Proportional Regression Model for Time to First Cardiac Event using Propensity Score

Predictor Estimate S.E. Hazard's Ratio Significance

\begin{tabular}{|c|c|c|c|c|}
\hline Age & -0.0063 & 0.0124 & 0.9937 & 0.609 \\
\hline Sex & -0.0110 & 0.1587 & 0.9890 & 0.944 \\
\hline $\begin{array}{l}\text { Appropriate/Inappropriate use } \\
\text { of Beta-blockers at post-AMI }\end{array}$ & 0.6844 & 0.2455 & 1.9826 & $0.005^{*}$ \\
\hline Propensity score & -0.8823 & 0.6585 & 0.4138 & 0.180 \\
\hline $\begin{array}{l}\text { Presence of absolute } \\
\text { contraindications }\end{array}$ & -0.2401 & 0.2774 & 0.7866 & 0.386 \\
\hline \multicolumn{5}{|l|}{$\begin{array}{l}\text { Procedures during the } \\
\text { incident AMI }\end{array}$} \\
\hline CABG & -0.9348 & 0.3239 & 0.3927 & $0.003 *$ \\
\hline PTCA & -0.3421 & 0.2261 & 0.7102 & 0.130 \\
\hline Length of stay & 0.0333 & 0.0168 & 1.0339 & $0.047 *$ \\
\hline \multicolumn{5}{|l|}{ Discharge Medications } \\
\hline Aspirin & 0.0420 & 0.2374 & 1.0429 & 0.859 \\
\hline ACE-inhibitors & 0.1529 & 0.1605 & 1.1652 & 0.340 \\
\hline $\begin{array}{l}\text { Calcium channel } \\
\text { blockers }\end{array}$ & 0.1497 & 0.1671 & 1.1615 & 0.370 \\
\hline Loop diuretics & 0.2045 & 0.1681 & 1.2269 & 0.223 \\
\hline Medication Possession Ratio & 0.0239 & 0.3094 & 1.0241 & 0.938 \\
\hline Day supply & -0.0011 & 0.0006 & 0.9989 & 0.126 \\
\hline
\end{tabular}

* Significant at $\mathrm{p} \leq$ 0.05, S.E: Standard error, AMI: Acute myocardial infarction, CABG: Coronary artery bypass graft, PTCA: Percutaneous transluminal coronary angioplasty, ACE: Angiotensin converting enzyme inhibitor 


\section{Discussion for Research Question 3}

The above analysis rejected the null hypothesis $\mathrm{C}$ that there was no significant relationship between appropriate use and inappropriate prescribing of beta-blockers and morbidity (time to first hospitalization due to a cardiovascular event). Thus, there was an association, but unlike mortality this association revealed that appropriate use was associated with a higher probability of a cardiovascular event in the post AMI 12-month period as compared to the inappropriate group. When the event was restricted to recurrent AMI, though the distribution was not significant, the survival curves illustrated that the association was similar to when all cardiac events were considered. The Coxproportional hazards model confirmed this association even after adjusting for all covariates. Appropriate beta-blocker use was still associated with greater morbidity. Additionally, the first model, where the co-variates included predictors of appropriate/inappropriate group, days supply was almost significant and was negatively associated with morbidity. This illustrates a conflict (appropriate use is associated with greater morbidity, but higher days supply is associated with lower morbidity). Thus, although there is a definite association between appropriate/inappropriate prescribing and morbidity, the above results do not clearly help interpret this association.

\section{Results for Research Question 4}

Utilization of services between the two groups was compared by exploring whether there was any difference in the number of hospitalizations, number of ER visits, number of physician visits, total length of stay, total charges and total reimbursed amounts in the follow-up period due to cardiac conditions. The descriptive statistics for 
these are listed in Table 14. The cardiac conditions were the same as those specified in Table 10. Two multiple regression models were performed for each of the utilization variables. The first model included those co-variates that were significant in predicting group status for appropriate/inappropriate beta-blocker receipt in addition to other covariates. The second model included propensity score instead of these predicting variables with other co-variates. The total charges and total reimbursed amounts variables were $\log$ transformed to decrease skewness of the data. The following utilization models were significant: number of hospital visits (both models), number of ER visits (both models), number of physicians visits (only the first model), length of stay (both models), log total charges (both models), and log total reimbursed amounts (both models) (Tables 15-26). The $\mathrm{R}^{2}$ values for the significant models ranged between 6 to $14 \%$. Thus, there was a lot of variance in the utilization variables that could not be explained by the independent variables. For interpretation of semilogarithmic models, the correction of Halverson and Palmquist with a modification by Kennedy was employed (Halverson, \& Palmquist, 1980; Kennedy, 1981). All models were checked for violation of assumptions associated with linear regression. The models had no problems with multicollinearity, heteroskedasticity and autocorrelation. The power of analysis for the models was $100 \%$. For these models, the variables that were significant in predicting the utilization variables are listed below:

Number of Hospital visits due to Cardiac Conditions (Group when significant is in bold):

First Model (Table 15): Appropriate/Inappropriate group of beta-blocker at post AMI, Number of non-cardiac admissions, Use of beta-blockers in the preperiod, CABG during incident AMI, and Length of stay during incident AMI. 
Propensity Score Model (Table 16): Appropriate/Inappropriate group of betablocker at post AMI, CABG during incident AMI, and Length of stay during incident AMI.

Number of ER visits due to Cardiac Conditions:

First Model (Table 17): Appropriate/Inappropriate group of beta-blocker at post AMI, Presence of absolute contraindications, Number of non-cardiac admissions, Use of beta-blockers in the pre-period, and Medication possession ratio (MPR).

Propensity Score Model (Table 18): Appropriate/Inappropriate group of betablocker at post AMI, Presence of absolute contraindications, and MPR.

Number of Physician visits due to Cardiac Conditions:

First Model (Table 19): Presence of hypertension, Number of non-cardiac admissions, and Use of beta-blockers in the pre-period.

Propensity Score Model (Table 20, model not significant): Presence of absolute contraindications during or before incident AMI.

Length of Stay due to Cardiac Conditions:

First Model (Table 21): Appropriate/Inappropriate group of beta-blocker at post AMI, Number of non-cardiac admissions, Use of beta-blockers in the preperiod, CABG during incident AMI, Length of stay during incident AMI, and Days supply.

Propensity Score Model (Table 22): Appropriate/Inappropriate group of betablocker at post AMI, CABG during incident AMI, Length of stay during incident AMI, and Days supply. 
Log Total Charges due to Cardiac Conditions:

First Model (Table 23): CABG during incident AMI.

Propensity Score Model (Table 24): CABG during incident AMI.

Log Total Reimbursed Amounts due to Cardiac Conditions:

First Model (Table 25): CABG during incident AMI and Length of stay during incident AMI.

Propensity Score Model (Table 26): CABG during incident AMI and Length of stay during incident AMI.

Appropriate/inappropriate use of beta-blockers post AMI was a significant predictor in some of the utilization variable models. It was a significant predictor for number of hospitalizations in the follow-up period, number of ER visits in the follow-up period, and length of stay in the follow-up period due to cardiac conditions. It did not affect the number of physician visits, charges and reimbursed amounts. Additionally, for the variables where it was significant, the beta coefficients in the models were positive, suggesting increased utilization of health care services for the appropriate group. Among the other significant predictors, higher number of non-cardiac admissions in the preperiod were associated with higher utilization, use of beta-blockers in the pre-period was associated with higher utilization, presence of any absolute contraindication was associated with higher utilization except for physicians visits where it was associated with lower utilization. Presence of hypertension was associated with fewer physician visits, $\mathrm{CABG}$ during incident $\mathrm{AMI}$ was associated with lower utilization but higher expenditures, longer length of stay during incident AMI was associated with lower 
utilization but higher expenditures, compliance variable (MPR) was associated with lower utilization and days supply was also associated with lower utilization. Propensity score was not significant in any of the models and use of propensity score did not affect significance of other variables. When analysis was repeated using beta-blocker prescribing at 30 and 60 days after the AMI to categorize appropriate and inappropriate prescribing, the results for utilization and expenditures did not change. 
Table 14: Utilization Variables

\begin{tabular}{lcc}
\hline Variables & Mean \pm S.D. & Range \\
\hline Number of Hospital Visits & $0.86 \pm 1.54$ & $0-11$ \\
Number of ER Visits & $0.23 \pm 0.88$ & $0-12$ \\
Number of Physicians Visits & $6.63 \pm 9.62$ & $0-64$ \\
Length of Stay & $4.65 \pm 9.77$ & $0-70$ \\
Total Charges & $\$ 17,258.50 \pm 33,146.57$ & $0-3,14,066.70$ \\
Total Reimbursed Amounts & $\$ 15,609.00 \pm 30,573.02$ & $0-2,77,218.00$ \\
\hline
\end{tabular}

ER: Emergency room, S.D: Standard deviation 
Table 15: Predictors for Number of Hospital Visits due to Cardiac Conditions

\begin{tabular}{|c|c|c|c|c|}
\hline Predictor & Estimate & S.E. & t-Statistic & Significance \\
\hline Age & -0.003 & 0.010 & 0.368 & 0.713 \\
\hline Sex & -0.013 & 0.152 & -0.087 & 0.931 \\
\hline $\begin{array}{l}\text { Appropriate/Inappropriate use } \\
\text { of beta-blockers at post-AMI }\end{array}$ & 0.829 & 0.228 & 3.627 & $0.000 *$ \\
\hline Hypertension & -0.180 & 0.155 & -1.162 & 0.246 \\
\hline $\begin{array}{l}\text { Presence of absolute } \\
\text { contraindications }\end{array}$ & 0.127 & 0.242 & 0.525 & 0.600 \\
\hline $\begin{array}{l}\text { Number of non-cardiac } \\
\text { admissions }\end{array}$ & 0.500 & 0.118 & 4.244 & $0.000 *$ \\
\hline \multicolumn{5}{|l|}{ Pre-AMI medications: } \\
\hline Beta-blockers & 0.486 & 0.189 & 2.575 & $0.010^{*}$ \\
\hline \multicolumn{5}{|l|}{$\begin{array}{l}\text { Procedures during the incident } \\
\text { AMI }\end{array}$} \\
\hline CABG & -0.647 & 0.260 & -2.489 & $0.013^{*}$ \\
\hline PTCA & 0.0007 & 0.205 & 0.004 & 0.997 \\
\hline Length of stay & -0.043 & 0.020 & 2.224 & $0.027 *$ \\
\hline \multicolumn{5}{|l|}{ Discharge Medications } \\
\hline Aspirin & -0.168 & 0.231 & -0.725 & 0.469 \\
\hline ACE-inhibitors & 0.128 & 0.157 & 0.816 & 0.415 \\
\hline $\begin{array}{l}\text { Calcium channel } \\
\text { blockers }\end{array}$ & 0.075 & 0.167 & 0.456 & 0.649 \\
\hline Loop diuretics & 0.225 & 0.165 & 1.366 & 0.173 \\
\hline Medication Possession Ratio & -0.129 & 0.309 & -0.419 & 0.676 \\
\hline
\end{tabular}


Table 15: Predictors for Number of Hospital Visits due to Cardiac Conditions (contd.)

\begin{tabular}{lcccc}
\hline Predictor & Estimate & S.E. & t-Statistic & Significance \\
\hline Days supply & -0.001 & 0.001 & -1.503 & 0.134 \\
& $\mathrm{R}^{2}=13.5 \%(\mathrm{~F}=3.798, \mathrm{p}=0.000 *), \mathrm{n}=407$ & \\
\hline
\end{tabular}

* Significant at $\mathrm{p} \leq 0.05$, S.E: Standard error, AMI: Acute myocardial infarction, CABG: Coronary artery bypass graft, PTCA: Percutaneous transluminal coronary angioplasty, ACE: Angiotensin converting enzyme inhibitor 
Table 16: Predictors for Number of Hospital Visits due to Cardiac Conditions using Propensity Score

\begin{tabular}{lllll} 
Predictor & Estimate & S.E. & t-Statistic & Significance \\
\hline Age & -0.007 & 0.012 & -0.611 & 0.542 \\
Sex & -0.096 & 0.156 & -0.622 & 0.534 \\
$\begin{array}{lllll}\text { Appropriate/Inappropriate use of } \\
\text { beta-blockers at post-AMI }\end{array}$ & 0.799 & 0.232 & 3.437 & $0.001^{*}$ \\
$\begin{array}{l}\text { Propensity score } \\
\text { Presence of absolute }\end{array}$ & -0.650 & 0.625 & -1.039 & 0.299 \\
$\begin{array}{l}\text { contraindications } \\
\text { Procedures during the incident }\end{array}$ & -0.026 & 0.267 & -0.101 & 0.920 \\
AMI & & & & \\
$\quad$ CABG & & & & \\
$\quad$ PTCA & -0.679 & 0.267 & -2.548 & $0.011^{*}$ \\
$\quad$ Length of stay & -0.111 & 0.209 & -0.532 & 0.595 \\
$\quad$ & -0.045 & 0.020 & 2.268 & $0.024^{*}$
\end{tabular}

Discharge Medications

\begin{tabular}{|c|c|c|c|c|}
\hline Aspirin & -0.215 & 0.237 & -0.909 & 0.364 \\
\hline ACE-inhibitors & 0.093 & 0.160 & 0.588 & 0.557 \\
\hline Calcium channel blockers & 0.177 & 0.169 & 1.047 & 0.296 \\
\hline Loop diuretics & 0.270 & 0.169 & 1.593 & 0.112 \\
\hline Medication Possession Ratio & -0.143 & 0.317 & -0.450 & 0.653 \\
\hline Days supply & -0.0008 & 0.001 & -1.114 & 0.266 \\
\hline
\end{tabular}

* Significant at $\mathrm{p} \leq$ 0.05, S.E: Standard error, AMI: Acute myocardial infarction, CABG: Coronary artery bypass graft, PTCA: Percutaneous transluminal coronary angioplasty, ACE: Angiotensin converting enzyme inhibitor 
Table 17: Predictors for Number of ER Visits due to Cardiac Conditions

\begin{tabular}{|c|c|c|c|c|}
\hline Predictor & Estimate & S.E. & t-Statistic & Significance \\
\hline Age & -0.006 & 0.006 & -1.188 & 0.236 \\
\hline Sex & -0.096 & 0.090 & -1.071 & 0.285 \\
\hline $\begin{array}{l}\text { Appropriate/Inappropriate use } \\
\text { of beta-blockers at post-AMI }\end{array}$ & 0.470 & 0.135 & 3.484 & $0.001 *$ \\
\hline Hypertension & -0.037 & 0.091 & -0.414 & 0.679 \\
\hline $\begin{array}{l}\text { Presence of absolute } \\
\text { contraindications }\end{array}$ & 0.320 & 0.143 & 2.242 & $0.026 *$ \\
\hline $\begin{array}{l}\text { Number of non-cardiac } \\
\text { admissions }\end{array}$ & 0.155 & 0.070 & 2.233 & $0.026 *$ \\
\hline \multicolumn{5}{|l|}{ Pre-AMI medications: } \\
\hline Beta-blockers & 0.220 & 0.111 & 1.977 & $0.049 *$ \\
\hline \multicolumn{5}{|l|}{$\begin{array}{l}\text { Procedures during the incident } \\
\text { AMI }\end{array}$} \\
\hline CABG & -0.057 & 0.153 & -0.376 & 0.707 \\
\hline PTCA & 0.095 & 0.121 & 0.794 & 0.427 \\
\hline Length of stay & -0.003 & 0.012 & -0.271 & 0.786 \\
\hline \multicolumn{5}{|l|}{ Discharge Medications } \\
\hline Aspirin & -0.049 & 0.137 & -0.361 & 0.718 \\
\hline ACE-inhibitors & -0.064 & 0.093 & -0.693 & 0.489 \\
\hline $\begin{array}{l}\text { Calcium channel } \\
\text { blockers }\end{array}$ & -0.0006 & 0.098 & -0.007 & 0.994 \\
\hline Loop diuretics & 0.165 & 0.097 & 1.697 & 0.090 \\
\hline Medication Possession Ratio & -0.474 & 0.182 & -2.598 & $.010^{*}$ \\
\hline
\end{tabular}


Table 17: Predictors for Number of ER Visits due to Cardiac Conditions (contd.)

\begin{tabular}{lcccc} 
Predictor & Estimate & S.E. & t-Statistic & Significance \\
\hline Days supply & -0.00004 & 0.000 & 0.095 & 0.924 \\
& $\mathrm{R}^{2}=8.5 \%(\mathrm{~F}=2.276, \mathrm{p}=0.003 *), \mathrm{n}=407$ &
\end{tabular}

* Significant at $\mathrm{p} \leq 0.05$, ER: Emergency room, S.E: Standard error, AMI: Acute myocardial infarction, CABG: Coronary artery bypass graft, PTCA: Percutaneous transluminal coronary angioplasty, ACE:

Angiotensin converting enzyme inhibitor 
Table 18: Predictors for Number of ER Visits due to Cardiac Conditions using Propensity Score

\begin{tabular}{lllll} 
Predictor & Estimate & S.E. & t-Statistic & Significance \\
\hline Age & 0.008 & 0.007 & -1.197 & 0.232 \\
Sex & -0.125 & 0.090 & -1.380 & 0.168 \\
$\begin{array}{l}\text { Appropriate/Inappropriate use of } \\
\text { beta-blockers at post-AMI }\end{array}$ & 0.460 & 0.135 & 3.417 & $0.001^{*}$ \\
$\begin{array}{l}\text { Propensity score } \\
\text { Presence of absolute }\end{array}$ & -0.006 & 0.363 & -0.017 & 0.987 \\
$\begin{array}{l}\text { contraindications } \\
\text { Procedures during the incident }\end{array}$ & 0.306 & 0.155 & 1.980 & $0.048^{*}$ \\
$\begin{array}{l}\text { AMI } \\
\quad \text { CABG }\end{array}$ & & & & \\
$\quad$ PTCA & -0.071 & 0.155 & -0.464 & 0.643 \\
$\quad$ Length of stay & 0.055 & 0.121 & 0.462 & 0.644 \\
$\quad-0.002$ & 0.012 & -0.200 & 0.842
\end{tabular}

Discharge Medications

$\begin{array}{lllll}\text { Aspirin } & -0.067 & 0.137 & -0.494 & 0.621 \\ \text { ACE-inhibitors } & -0.076 & 0.093 & -0.827 & 0.408 \\ \text { Calcium channel blockers } & 0.035 & 0.098 & 0.367 & 0.714 \\ \text { Loop diuretics } & 0.184 & 0.098 & 1.869 & 0.062 \\ \text { Medication Possession Ratio } & -0.481 & 0.184 & -2.619 & 0.009 * \\ \text { Days supply } & 0.0001 & 0.000 & 0.332 & 0.740 \\ & \mathrm{R}^{2}=6.4 \%(\mathrm{~F}=1.935, \mathrm{p}=0.022 *), \mathrm{n}=407 & \\ \end{array}$

\footnotetext{
* Significant at $\mathrm{p} \leq 0.05$, ER: Emergency room, S.E: Standard error, AMI: Acute myocardial infarction, CABG: Coronary artery bypass graft, PTCA: Percutaneous transluminal coronary angioplasty, ACE: Angiotensin converting enzyme inhibitor
} 
Table 19: Predictors for Number of Physician Visits due to Cardiac Conditions

\begin{tabular}{|c|c|c|c|c|}
\hline Predictor & Estimate & S.E. & t-Statistic & Significance \\
\hline Age & 0.008 & 0.062 & -0.136 & 0.892 \\
\hline Sex & -0.603 & 0.980 & -0.615 & 0.539 \\
\hline $\begin{array}{l}\text { Appropriate/Inappropriate use } \\
\text { of beta-blockers at post-AMI }\end{array}$ & 2.406 & 1.469 & 1.639 & 0.102 \\
\hline Hypertension & -1.995 & 0.995 & -2.004 & $0.046^{*}$ \\
\hline $\begin{array}{l}\text { Presence of absolute } \\
\text { contraindications }\end{array}$ & -2.984 & 1.554 & -1.920 & 0.056 \\
\hline $\begin{array}{l}\text { Number of non-cardiac } \\
\text { admissions }\end{array}$ & 2.248 & 0.757 & 2.968 & $0.003 *$ \\
\hline \multicolumn{5}{|l|}{ Pre-AMI medications: } \\
\hline Beta-blockers & 2.885 & 1.213 & 2.379 & $0.018 *$ \\
\hline \multicolumn{5}{|l|}{$\begin{array}{l}\text { Procedures during the } \\
\text { incident AMI }\end{array}$} \\
\hline $\mathrm{CABG}$ & -3.061 & 1.671 & -1.831 & 0.068 \\
\hline PTCA & -1.560 & 1.313 & -1.186 & 0.236 \\
\hline Length of stay & 0.061 & 0.127 & 0.489 & 0.625 \\
\hline \multicolumn{5}{|l|}{ Discharge Medications } \\
\hline Aspirin & -1.837 & 1.487 & -1.235 & 0.217 \\
\hline ACE-inhibitors & 0.570 & 1.010 & 0.564 & 0.573 \\
\hline $\begin{array}{l}\text { Calcium channel } \\
\text { Blockers }\end{array}$ & 0.250 & 1.071 & 0.234 & 0.815 \\
\hline Loop diuretics & 0.070 & 1.060 & -0.066 & 0.947 \\
\hline Medication Possession Ratio & 1.999 & 1.987 & 1.006 & 0.315 \\
\hline
\end{tabular}


Table 19: Predictors for Number of Physician Visits due to Cardiac Conditions (contd.)

\begin{tabular}{llcll}
\hline Predictor & Estimate & S.E. & t-Statistic & Significance \\
\hline Days supply & -0.008 & 0.005 & -1.924 & 0.055 \\
& $\mathrm{R}^{2}=8.6 \%(\mathrm{~F}=2.299, \mathrm{p}=0.003 *), \mathrm{n}=407$ & \\
\hline
\end{tabular}

* Significant at $\mathrm{p} \leq 0.05$, S.E: Standard error, AMI: Acute myocardial infarction, CABG: Coronary artery bypass graft, PTCA: Percutaneous transluminal coronary angioplasty, ACE: Angiotensin converting enzyme inhibitor 
Table 20: Predictors for Number of Physician Visits due to Cardiac Conditions using Propensity Score

\begin{tabular}{lllll} 
Predictor & Estimate & S.E. & t-Statistic & Significance \\
\hline Age & -0.052 & 0.074 & -0.706 & 0.480 \\
Sex & -0.883 & 0.991 & -0.891 & 0.374 \\
$\begin{array}{l}\text { Appropriate/Inappropriate use of } \\
\text { beta-blockers at post-AMI }\end{array}$ & 1.977 & 1.481 & 1.335 & 0.183 \\
$\begin{array}{l}\text { Propensity score } \\
\text { Presence of absolute }\end{array}$ & -1.941 & 3.984 & -0.487 & 0.626 \\
contraindications & -3.571 & 1.698 & -2.103 & $0.036^{*}$ \\
$\begin{array}{l}\text { Procedures during the incident } \\
\text { AMI }\end{array}$ & & & & \\
$\quad$ CABG & & & & \\
$\quad$ PTCA & -3.139 & 1.699 & -1.848 & 0.065 \\
$\quad$ Length of stay & -2.140 & 1.330 & -1.609 & 0.108 \\
$\quad 0.071$ & 0.129 & 0.552 & 0.581
\end{tabular}

Discharge Medications

$\begin{array}{lllll}\text { Aspirin } & -2.044 & 1.510 & -1.353 & 0.177 \\ \text { ACE-inhibitors } & 0.194 & 1.017 & 0.191 & 0.849 \\ \text { Calcium channel blockers } & 0.641 & 1.078 & 0.594 & 0.553 \\ \text { Loop diuretics } & 0.231 & 1.079 & 0.214 & 0.831 \\ \text { Medication Possession Ratio } & 2.027 & 2.020 & 1.003 & 0.316 \\ \text { Days supply } & -0.007 & 0.005 & -1.554 & 0.121 \\ & \mathrm{R}^{2}=4.9 \%(\mathrm{~F}=1.441, \mathrm{p}=0.131), \mathrm{n}=407 & \\ \end{array}$

* Significant at $\mathrm{p} \leq 0.05$, S.E: Standard error, AMI: Acute myocardial infarction, CABG: Coronary artery bypass graft, PTCA: Percutaneous transluminal coronary angioplasty, ACE: Angiotensin converting enzyme inhibitor 
Table 21: Predictors for Length of Stay due to Cardiac Conditions

\begin{tabular}{|c|c|c|c|c|}
\hline Predictor & Estimate & S.E. & t-Statistic & Significance \\
\hline Age & 0.055 & 0.062 & 0.890 & 0.374 \\
\hline Sex & -0.173 & 0.977 & -0.177 & 0.859 \\
\hline $\begin{array}{l}\text { Appropriate/Inappropriate use } \\
\text { of beta-blockers at post-AMI }\end{array}$ & 3.417 & 1.464 & 2.334 & $0.020 *$ \\
\hline Hypertension & -0.427 & 0.992 & -0.430 & 0.667 \\
\hline $\begin{array}{l}\text { Presence of absolute } \\
\text { contraindications }\end{array}$ & 0.785 & 1.549 & 0.506 & 0.613 \\
\hline $\begin{array}{l}\text { Number of non-cardiac } \\
\text { admissions }\end{array}$ & 1.695 & 0.755 & 2.245 & $0.025 *$ \\
\hline \multicolumn{5}{|l|}{ Pre-AMI medications: } \\
\hline Beta-blockers & 2.343 & 1.209 & 1.939 & $0.053 *$ \\
\hline \multicolumn{5}{|l|}{$\begin{array}{l}\text { Procedures during the incident } \\
\text { AMI }\end{array}$} \\
\hline CABG & -5.510 & 1.666 & -3.307 & $0.001 *$ \\
\hline PTCA & -1.200 & 1.311 & -0.915 & 0.361 \\
\hline Length of stay & 0.425 & 0.126 & 3.368 & $0.001 *$ \\
\hline \multicolumn{5}{|l|}{ Discharge Medications } \\
\hline Aspirin & 0.316 & 1.483 & 0.213 & 0.831 \\
\hline ACE-inhibitors & 0.293 & 1.007 & 0.291 & 0.771 \\
\hline $\begin{array}{l}\text { Calcium channel } \\
\text { Blockers }\end{array}$ & -0.020 & 1.068 & -0.019 & 0.985 \\
\hline Loop diuretics & 1.561 & 1.057 & 1.476 & 0.141 \\
\hline Medication Possession Ratio & 1.142 & 1.981 & 0.576 & 0.565 \\
\hline
\end{tabular}


Table 21: Predictors for Length of Stay due to Cardiac Conditions (contd.)

\begin{tabular}{lcccc}
\hline Predictor & Estimate & S.E. & t-Statistic & Significance \\
\hline Days supply & -0.012 & 0.005 & -2.617 & $0.009^{*}$ \\
& $\mathrm{R}^{2}=12.0 \%\left(\mathrm{~F}=3.335, \mathrm{p}=0.000^{*}\right), \mathrm{n}=407$ & \\
\hline
\end{tabular}

* Significant at $\mathrm{p} \leq 0.05$, S.E: Standard error, AMI: Acute myocardial infarction, CABG: Coronary artery bypass graft, PTCA: Percutaneous transluminal coronary angioplasty, ACE: Angiotensin converting enzyme inhibitor 
Table 22: Predictors for Length of Stay due to Cardiac Conditions using Propensity Score

\section{Predictor}

Age
Sex
Appropriate/Inappropriate use o
beta-blockers at post-AMI
Propensity score
Presence of absolute
contraindications
Procedures during the incident
AMI
Estimate S.E. t-Statistic Significance

$\begin{array}{llll}0.033 & 0.073 & 0.455 & 0.649\end{array}$

$-0.492$

0.979

$-0.503$

0.615

3.341

1.463

2.284

$0.023^{*}$

$-0.818$

3.936

$-0.208$

0.835

0.512

1.678

0.305

0.760

$\begin{array}{lcccc}\text { CABG } & -5.652 & 1.678 & -3.368 & 0.001^{*} \\ \text { PTCA } & -1.624 & 1.314 & -1.236 & 0.217 \\ \text { Length of stay } & 0.433 & 0.127 & 3.404 & 0.001^{*}\end{array}$

Discharge Medications

\begin{tabular}{|c|c|c|c|c|}
\hline Aspirin & 0.105 & 1.492 & 0.071 & 0.944 \\
\hline ACE-inhibitors & 0.174 & 1.005 & 0.173 & 0.863 \\
\hline Calcium channel blockers & 0.376 & 1.065 & 0.353 & 0.724 \\
\hline Loop diuretics & 1.742 & 1.066 & 1.634 & 0.103 \\
\hline Medication Possession Ratio & 1.070 & 1.996 & 0.536 & 0.592 \\
\hline Days supply & -0.010 & 0.005 & -2.372 & $0.018^{*}$ \\
\hline
\end{tabular}

\footnotetext{
* Significant at $\mathrm{p} \leq 0.05$, S.E: Standard error, AMI: Acute myocardial infarction, CABG: Coronary artery bypass graft, PTCA: Percutaneous transluminal coronary angioplasty, ACE: Angiotensin converting enzyme inhibitor
} 
Table 23: Predictors for Log Charges due to Cardiac Conditions

\begin{tabular}{|c|c|c|c|c|}
\hline Predictor & Estimate & S.E. & t-Statistic & Significance \\
\hline Age & -0.035 & 0.039 & -0.893 & 0.372 \\
\hline Sex & 2.311 & 0.618 & 1.664 & 0.097 \\
\hline $\begin{array}{l}\text { Appropriate/Inappropriate use } \\
\text { of beta-blockers at post-AMI }\end{array}$ & 1.096 & 0.926 & 0.562 & 0.574 \\
\hline Hypertension & 1.138 & 0.628 & 0.521 & 0.603 \\
\hline $\begin{array}{l}\text { Presence of absolute } \\
\text { contraindications }\end{array}$ & 0.139 & 0.980 & -1.521 & 0.129 \\
\hline $\begin{array}{l}\text { Number of non-cardiac } \\
\text { admissions }\end{array}$ & 0.748 & 0.478 & 1.566 & 0.118 \\
\hline \multicolumn{5}{|l|}{ Pre-AMI medications: } \\
\hline Beta-blockers & 1.899 & 0.765 & 1.222 & 0.223 \\
\hline \multicolumn{5}{|l|}{$\begin{array}{l}\text { Procedures during the } \\
\text { incident AMI }\end{array}$} \\
\hline $\mathrm{CABG}$ & 0.011 & 1.054 & -3.715 & $0.000 *$ \\
\hline PTCA & 0.806 & 0.829 & 0.154 & 0.878 \\
\hline Length of stay & 0.144 & 0.080 & 1.808 & 0.071 \\
\hline
\end{tabular}

Discharge Medications

\begin{tabular}{|c|c|c|c|c|}
\hline Aspirin & 1.629 & 0.938 & 0.990 & 0.323 \\
\hline ACE-inhibitors & 0.854 & 0.637 & 0.072 & 0.942 \\
\hline $\begin{array}{l}\text { Calcium channel } \\
\text { blockers }\end{array}$ & 1.798 & 0.675 & 1.206 & 0.228 \\
\hline Loop diuretics & 1.539 & 0.669 & 0.980 & 0.328 \\
\hline cation Possession Ratio & 1.311 & 1.253 & 1.046 & 0.296 \\
\hline
\end{tabular}


Table 23: Predictors for Log Charges due to Cardiac Conditions (contd.)

\begin{tabular}{lcccc} 
Predictor & Estimate & S.E. & t-Statistic & Significance \\
\hline Days supply & -0.003 & 0.003 & -1.215 & 0.225 \\
& $\mathrm{R}^{2}=7.7 \%\left(\mathrm{~F}=2.050, \mathrm{p}=0.010^{*}\right), \mathrm{n}=407$ &
\end{tabular}

* Significant at $\mathrm{p} \leq 0.05$, S.E: Standard error, AMI: Acute myocardial infarction, CABG: Coronary artery bypass graft, PTCA: Percutaneous transluminal coronary angioplasty, ACE: Angiotensin converting enzyme inhibitor 
Table 24: Predictors for Log Charges due to Cardiac Conditions Using Propensity Score

\begin{tabular}{lcccl} 
Predictor & Estimate & S.E. & t-Statistic & Significance \\
\hline Age & -0.029 & 0.046 & -0.631 & 0.528 \\
Sex & 2.186 & 0.616 & 1.415 & 0.158 \\
$\begin{array}{l}\text { Appropriate/Inappropriate use of } \\
\text { beta-blockers at post-AMI }\end{array}$ & 1.136 & 0.921 & 0.600 & 0.549 \\
$\begin{array}{l}\text { Propensity score } \\
\begin{array}{l}\text { Presence of absolute } \\
\text { contraindications }\end{array}\end{array}$ & 0.955 & 2.477 & 0.386 & 0.700 \\
& 0.144 & 1.056 & -1.303 & 0.193
\end{tabular}

Procedures during the incident

AMI

$\begin{array}{lllll}\text { CABG } & 0.010 & 1.056 & -3.818 & 0.000^{*} \\ \text { PTCA } & 0.669 & 0.827 & -0.072 & 0.943 \\ \text { Length of stay } & 0.150 & 0.080 & 1.868 & 0.062\end{array}$

Discharge Medications

$\begin{array}{lcccc}\text { Aspirin } & 1.495 & 0.939 & 0.898 & 0.370 \\ \text { ACE-inhibitors } & 0.779 & 0.632 & 0.078 & 0.938 \\ \text { Calcium channel blockers } & 2.233 & 0.670 & 1.534 & 0.126 \\ \text { Loop diuretics } & 1.668 & 0.671 & 1.099 & 0.272 \\ \text { Medication Possession Ratio } & 1.219 & 1.256 & 0.970 & 0.332 \\ \text { Days supply } & -0.003 & .003 & -1.126 & 0.261 \\ & \\ \mathrm{R}^{2}=6.7 \%(\mathrm{~F}=2.003, \mathrm{p}=0.017 *), \mathrm{n}=407 & \end{array}$

* Significant at $\mathrm{p} \leq 0.05$, S.E: Standard error, AMI: Acute myocardial infarction, CABG: Coronary artery bypass graft, PTCA: Percutaneous transluminal coronary angioplasty, ACE: Angiotensin converting enzyme inhibitor 
Table 25: Predictors for Log Reimbursed Amounts due to Cardiac Conditions

\begin{tabular}{|c|c|c|c|c|}
\hline Predictor & Estimate & S.E. & t-Statistic & Significance \\
\hline Age & -0.041 & 0.039 & -1.044 & 0.297 \\
\hline Sex & 2.406 & 0.621 & 1.725 & 0.085 \\
\hline $\begin{array}{l}\text { Appropriate/Inappropriate use } \\
\text { of beta-blockers at post-AMI }\end{array}$ & 1.380 & 0.930 & 0.812 & 0.418 \\
\hline Hypertension & 1.110 & 0.630 & 0.481 & 0.631 \\
\hline $\begin{array}{l}\text { Presence of absolute } \\
\text { contraindications }\end{array}$ & 0.164 & 0.984 & -1.343 & 0.180 \\
\hline $\begin{array}{l}\text { Number of non-cardiac } \\
\text { admissions }\end{array}$ & 0.846 & 0.480 & 1.763 & 0.079 \\
\hline \multicolumn{5}{|l|}{ Pre-AMI medications: } \\
\hline Beta-blockers & 2.032 & 0.768 & 1.307 & 0.192 \\
\hline \multicolumn{5}{|l|}{$\begin{array}{l}\text { Procedures during the } \\
\text { incident AMI }\end{array}$} \\
\hline CABG & 0.011 & 1.059 & -3.708 & $0.000 *$ \\
\hline PTCA & 0.763 & 0.833 & 0.093 & 0.926 \\
\hline Length of stay & 0.159 & 0.080 & 1.981 & $0.048 *$ \\
\hline \multicolumn{5}{|l|}{ Discharge Medications } \\
\hline Aspirin & 1.152 & 0.942 & 0.622 & 0.534 \\
\hline ACE-inhibitors & 0.816 & 0.640 & 0.004 & 0.997 \\
\hline $\begin{array}{l}\text { Calcium channel } \\
\text { blockers }\end{array}$ & 1.639 & 0.678 & 1.067 & 0.287 \\
\hline Loop diuretics & 1.403 & 0.672 & 0.841 & 0.401 \\
\hline Medication Possession Ratio & 1.415 & 1.258 & 1.124 & 0.262 \\
\hline
\end{tabular}


Table 25: Predictors for Log Reimbursed Amounts due to Cardiac Conditions (contd.)

\begin{tabular}{lcccc}
\hline Predictor & Estimate & S.E. & t-Statistic & Significance \\
\hline Days supply & -0.003 & 0.003 & -1.268 & 0.206 \\
& $\mathrm{R}^{2}=8.0 \%(\mathrm{~F}=2.134, \mathrm{p}=0.007 *), \mathrm{n}=407$ & \\
\hline
\end{tabular}

* Significant at $\mathrm{p} \leq$ 0.05, S.E: Standard error, AMI: Acute myocardial infarction, CABG: Coronary artery bypass graft, PTCA: Percutaneous transluminal coronary angioplasty, ACE: Angiotensin converting enzyme inhibitor 
Table 26: Predictors for Log Reimbursed Amounts due to Cardiac Conditions using Propensity Score

\begin{tabular}{lllll} 
Predictor & Estimate & S.E. & t-Statistic & Significance \\
\hline Age & -0.003 & 0.046 & -0.795 & 0.427 \\
Sex & 2.027 & 0.620 & 1.451 & 0.148 \\
$\begin{array}{l}\text { Appropriate/Inappropriate use of } \\
\text { beta-blockers at post-AMI }\end{array}$ & 1.422 & 0.926 & 0.843 & 0.400 \\
$\begin{array}{l}\text { Propensity score } \\
\text { Presence of absolute }\end{array}$ & 0.863 & 2.490 & 0.346 & 0.729 \\
$\begin{array}{l}\text { contraindications } \\
\text { Procedures during the incident }\end{array}$ & 0.165 & 1.062 & -1.160 & 0.247 \\
AMI & & & & \\
$\quad$ CABG & & & & \\
$\quad$ PTCA & 0.009 & 1.062 & -3.813 & $0.000^{*}$ \\
$\quad$ Length of stay & 0.621 & 0.831 & -0.158 & 0.875 \\
$\quad 0.164$ & 0.080 & 2.044 & $0.042^{*}$
\end{tabular}

Discharge Medications

\begin{tabular}{lcccc} 
Aspirin & 1.049 & 0.944 & 0.523 & 0.601 \\
ACE-inhibitors & 0.816 & 0.636 & 0.000 & 1.000 \\
Calcium channel blockers & 2.072 & 0.674 & 1.419 & 0.157 \\
Loop diuretics & 1.534 & 0.675 & 0.973 & 0.331 \\
Medication Possession Ratio & 1.318 & 1.263 & 1.044 & 0.297 \\
Days supply & -0.003 & 0.003 & -1.166 & 0.244 \\
& $\mathrm{R}^{2}=6.7 \%\left(\mathrm{~F}=2.031, \mathrm{p}=0.015^{*}\right), \mathrm{n}=407$ & \\
\hline
\end{tabular}

\footnotetext{
* Significant at $\mathrm{p} \leq 0.05$, S.E: Standard error, AMI: Acute myocardial infarction, CABG: Coronary artery bypass graft, PTCA: Percutaneous transluminal coronary angioplasty, ACE: Angiotensin converting enzyme inhibitor
} 


\section{Discussion for Research Question 4}

The null hypothesis D is rejected and appropriate/inappropriate use of betablockers does affect utilization and null hypothesis $\mathrm{E}$ is not rejected and appropriate/inappropriate use of beta-blockers does not affect expenditures. Appropriate/Inappropriate prescribing of beta-blockers following an AMI, did affect utilization such as hospital visits, ER visits and length of stay in the follow-up duration. This relationship was similar to the morbidity results, which demonstrated that appropriate use was associated with greater hazards for cardiac hospitalization. In other words, appropriate use was associated with increased utilization and longer length of stay due to cardiac visits. Physician visits and expenditures were not affected by appropriate/inappropriate use.

A further investigation to explain this association was undertaken. This revealed that the appropriate group had a significantly higher percentage $(\mathrm{p}=0.003, \mathrm{n}=412)$ of patients with hypertension (50.4\%) - one of the important risk factors for cardiac problems, compared to the inappropriate group (35.3\%). A recent study has revealed that during the 6-month period after a successful PTCA, patients with hypertension have significantly higher adverse event rates that those without hypertension (Tsang, Sheppard, Mak, Brown, Huynh et al., 2002). Hence, it is possible that the patients in the appropriate group had worse outcomes (higher utilization-hospitalization, ER visits and length of stay and lesser time to first cardiac event in research question 3) due to a higher percentage of hypertensive patients.

Additionally, the two groups were compared for other anti-anginal drug use such as nitroglycerin and calcium channel blocker. Due to the unavailability of other 
information such as Killip class, ejection fraction (EF), and creatinine phosphokinase levels (available in patient charts but not available in patient claims data) this was considered to be a proxy for severity of incident AMI. This revealed that the appropriate group had a significantly higher $(\mathrm{p}=0.001)$ percentage $(74.4 \%)$ of patients on other antianginals compared to the inappropriate group (60.3\%). Both the above characteristics (hypertension and anti-anginal use) insinuate that the appropriate group was probably in a worse health state during the incident AMI compared to the inappropriate group. Hence, higher utilization in the appropriate group could be because of their more severe illness rather than beta-blocker use.

An important observation is that beta-blockers did have significantly lower allcause mortality and a non-significantly lower cardiac mortality. It is possible that therapy actually postponed death in severe patients. This however, resulted in them being alive and utilizing more resources which resulted in higher morbidity and utilization.

Among the other significant predictors for utilization, higher non-cardiac admissions before the incident AMI, presence of absolute contraindications, and use of beta-blockers in the pre-period were associated with higher utilization. These variables suggest that patients with more co-morbidities, and thus worse health states, had more utilization after the AMI. In other words, presence of certain co-morbidties can increase the risk for the patient to suffer from more cardiac illness. Presence of hypertension was a significant predictor in the number of physician visits model and was negatively associated with number of physician visits. Similarly, CABG during incident AMI and higher length of stay during incident AMI was associated with lower utilization. These findings are difficult to interpret. Logically, a person with hypertension would have more 
physician visits, compared to a non-hypertensive patient. Also, a patient with higher length of stay and/or CABG during incident AMI would have higher utilization due to higher severity of the incident AMI. Thus, these results identify more gaps due to unavailability of other information that would be present on patient charts. MPR or compliance and days supply, were both associated with lower utilization. Thus, for those patients who received beta-blockers, treatment did have a protective effect and thus they had lower utilization.

The above analyses indicated that there was a definite association between appropriate/inappropriate use and utilization of health care services. There was higher utilization in the appropriately prescribed group and this could be due to higher severity of the patients with AMI in this group compared to the inappropriate group. This phase also identified inability of claims data to recognize severity levels of patients' conditions as a limitation for studying utilization of health care services due to cardiac conditions in the follow-up period after an AMI.

\section{Phase II}

Phase II of the study involved assessing effects of physicians' knowledge of contraindications and willingness to prescribe on their prescribing behavior for post-AMI beta-blocker therapy. It involved surveying physicians associated with post-AMI care. A mailing list was obtained from West Virginia Medical Board. The mailing list had a total of 1,151 physicians from the specialties of cardiology, internal medicine, family practice and general practice. A survey instrument developed for this project was mailed to each physician. Table 27 illustrates the response rate analysis. Of the 1,151 surveys mailed, 
132 questionnaires were returned because of incomplete or wrong addresses, and death or retirement of the physician. Thus, 1,019 surveys were assumed to have reached the respondents. A total of 281 responses were obtained giving a response rate of $27.57 \%$. Twenty surveys were excluded because they had more than $50 \%$ incomplete item responses, thereby reducing the response rate to $25.61 \%$. A higher response rate is always desirable, but this response rate is comparable to other studies involving physician population surveys. For example a recent survey by Zeiler et al., had a response rate of $20 \%$ and Chao had a response rate of 17.6\%. (Zierler, Meissner, Cain \& Strandness, 2002 ; Chao, 2002). 
Table 27: Response Rate Calculations

Initial survey population $\quad 1,151$

Wrong addresses/dead/retired 132

Effective population reached $\quad 1,019$

Incomplete responses $\quad 20$

Response rate $261 / 1,019=25.61 \%$ 


\section{Non-response Analysis}

The non-response survey (Appendix G) was mailed to 738 physicians who did not respond to one of the three survey mailings. A total of 121 completed surveys were returned, thus giving a response rate of approximately $16.40 \%$. Table 28 depicts the reasons the respondents gave for not responding to the main survey. The most common reasons were: not enough time to complete the survey (30.6\%), followed by "don't like to respond to survey" (17.4\%), and survey was too long (14.9\%). Among other reasons, survey was misplaced (9.9\%), and forgot about survey (7.4\%) were more common. A few reported topic irrelevant (5.0\%), not interested (4.1\%), not enough incentive (3.3\%), did not receive survey (2.5\%), and not enough information (2.5\%).

Table 29 presents demographic and practice characteristics of the respondents to the non-response survey. Of the 89 respondents, who responded to the question, 65 were males and 24 were females. Also, $17.9 \%$ were less than 40 years of age, $34.5 \%$ were 40 $49,28.6 \%$ were $50-59$, and $19.0 \%$ were 60 or older. The mean age was $50.55(\mathrm{SD}=$ $11.80)$ years. The distribution of the respondents in the different specialty were; $2.2 \%$ cardiology, $30.3 \%$ internal medicine, $39.3 \%$ family practice, $5.6 \%$ general practice and $22.5 \%$ others. Of the 63 respondents who answered the question on year of board certification, $52.4 \%$ were certified before or during 1990 , and $47.6 \%$ were certified after 1990. A total of $43.9 \%$ described their primary practice place as solo, $32.9 \%$ as groupbased, $11.0 \%$ as hospital-based, $11.0 \%$ as university-affiliated hospital, and $1.2 \%$ as other. The mean number of new AMI patients visiting every month was 2.02 and the mean number of repeat AMI patients visiting every month was 2.63. 
The respondents and non-respondents were compared on the basis of their demographic and practice characteristics. The variables compared were age, gender, specialty, year of board certification, practice site, number of new and repeat AMI patients treated. Chi-squares and t-tests were used for comparison of these variables. The result of this analysis is reported in Table 30. The respondents were not different from the non-respondents on age, year of board certification, practice site and average number of new AMI patients treated per month. The respondents and non-respondents were significantly different on gender, specialty, and average number of repeat AMI patients treated per month. A higher percent of non-respondents were females compared to the respondents. There were more physicians among the non-respondents who reported to belong to other specialty compared to respondents. The respondents reported a higher average number of repeat AMI patients treated per month compared to nonrespondents. Thus, results of the study should be interpreted with caution. 
Table 28: Reasons for Not Responding to the Study Survey

\begin{tabular}{lll}
\hline Reasons & N (121) & Percent (\%) \\
\hline Not enough time to complete survey & 37 & 30.6 \\
Don't like to respond to survey & 21 & 17.4 \\
Survey was too long & 18 & 14.9 \\
Survey was misplaced & 12 & 9.9 \\
Forgot about survey & 9 & 7.4 \\
Topic was irrelevant & 6 & 5.0 \\
Not interested in such studies & 5 & 4.1 \\
Not enough incentive to complete survey & 4 & 3.3 \\
Did not receive the survey & 3 & 2.5 \\
Not enough information about study & 3 & 2.5 \\
Other (retired, don't treat AMI, leave, sick) & 46 & 38.0 \\
\hline
\end{tabular}

N: Number of physicians, AMI: Acute myocardial infarction

Multiple responses were checked by the responders of the non-response survey, hence total greater than $100 \%$ 
Table 29: Demographic and Practice Characteristics of Respondents of the Nonresponse Survey

$\begin{array}{lll}\text { Characteristics } & \text { N } & \text { Percent }\end{array}$

Age

Less than 40 years

40-49 years

50-59 years

24

28.6

60 years or older

16

19.0

Average age \pm S.D.

$50.55 \pm 11.80$

Gender

Males

65

Females

24

27.0

Specialty

Cardiology

Internal Medicine

Family Practice

General Practice

Other

Year of Board Certification

$$
\begin{aligned}
& \leq 1990 \\
& >1990
\end{aligned}
$$

Primary Practice site

Solo, office-based
30

47.6

30.3

39.3

5.6

22.5

20

36

43.9 
Table 29: Demographic and Practice Characteristics of Respondents of the Nonresponse Survey (contd.)

\begin{tabular}{lll}
\hline Characteristics & N & Percent \\
\hline $\begin{array}{l}\text { Group, office-based } \\
\text { Hospital-based }\end{array}$ & 27 & 32.9 \\
$\quad$ University-affiliated hospital based & 9 & 11.0 \\
$\quad$ Other & 1 & 11.0 \\
$\begin{array}{l}\text { Average number of new AMI patients } \\
\text { Mean (S.D.), Min-Max }\end{array}$ & $2.02 \pm 3.53$ & 1.2 \\
$\begin{array}{l}\text { Average number of repeat AMI patients } \\
\text { Mean (S.D.), Min-Max }\end{array}$ & $2.63 \pm 4.99$ & $0-20$ \\
& & $0-30$ \\
\hline
\end{tabular}

N: Number of physicians, S.D: Standard deviation, AMI: Acute myocardial infarction, Min: Minimum value, Max: Maximum value 
Table 30: Analysis of Non-response Bias for Demographic and Practice Characteristics

\begin{tabular}{|c|c|c|c|c|}
\hline Characteristics & Respondents & $\begin{array}{l}\text { Non- } \\
\text { respondents }\end{array}$ & $\begin{array}{l}\text { Test } \\
\text { Statistic }\end{array}$ & $\begin{array}{l}\text { Significance } \\
\mathbf{p}\end{array}$ \\
\hline Age Mean (S.D.) & $49.23 \pm 10.96$ & $50.55 \pm 11.80$ & $\mathrm{t}=-0.938$ & 0.349 \\
\hline Gender & & & $\chi^{2}=3.84$ & $0.050 *$ \\
\hline Males & 82.6 & 73.0 & & \\
\hline Females & 17.4 & 27.0 & & \\
\hline Specialty & & & $\chi^{2}=22.23$ & $0.000 *$ \\
\hline Cardiology & 9.7 & 2.2 & & \\
\hline Internal Medicine & 32.6 & 30.3 & & \\
\hline Family Practice & 47.7 & 39.3 & & \\
\hline General Practice & 3.5 & 5.6 & & \\
\hline Other & 6.6 & 22.5 & & \\
\hline $\begin{array}{l}\text { Year of Board } \\
\text { Certification }\end{array}$ & & & $\chi^{2}=0.018$ & 0.892 \\
\hline$\leq 1990$ & 53.4 & 52.4 & & \\
\hline$>1990$ & 46.6 & 47.6 & & \\
\hline Practice site & & & $\chi^{2}=5.334$ & 0.255 \\
\hline Solo, office-based & 35.1 & 43.9 & & \\
\hline $\begin{array}{l}\text { Group, office- } \\
\text { based }\end{array}$ & 33.6 & 32.9 & & \\
\hline Hospital-based & 14.7 & 11.0 & & \\
\hline $\begin{array}{l}\text { University- } \\
\text { affiliated } \\
\text { hospital-based }\end{array}$ & 10.0 & 11.0 & & \\
\hline
\end{tabular}


Table 30: Analysis of Non-response Bias for Demographic and Practice Characteristics (contd.)

\begin{tabular}{|c|c|c|c|c|}
\hline Characteristics & Respondents & $\begin{array}{l}\text { Non- } \\
\text { respondents }\end{array}$ & $\begin{array}{l}\text { Test } \\
\text { Statistic }\end{array}$ & $\begin{array}{l}\text { Significance } \\
\text { p }\end{array}$ \\
\hline Other & 6.6 & 1.2 & & \\
\hline $\begin{array}{l}\text { Average number of new } \\
\text { AMI patients } \\
\text { Mean (S.D.) }\end{array}$ & $3.36 \pm 5.40$ & $2.02 \pm 3.53$ & $t=1.894$ & 0.059 \\
\hline $\begin{array}{l}\text { Average number of } \\
\text { repeat AMI patients } \\
\text { Mean (S.D.) }\end{array}$ & $9.64 \pm 33.92$ & $2.63 \pm 4.99$ & $\mathrm{t}=3.005$ & $0.003^{*}$ \\
\hline
\end{tabular}

* Significant at $\mathrm{p} \leq 0.05$, S.D: Standard deviation, AMI: Acute myocardial infarction 


\section{Demographic and Practice Characteristics of Physicians in Phase II}

Demographic and practice characteristics of the respondents are presented in Table 31. Of the respondents, $214(82.6 \%)$ were males and $45(17.4 \%)$ were females. The average age was 49.23 years $(\mathrm{SD}=10.96)$. Approximately $10 \%$ belonged to the specialty of cardiology, $33 \%$ belonged to internal medicine, $48 \%$ belonged to family practice, $4 \%$ belonged to general practice, and $7 \%$ reported other specialties. The year of board certification was divided into two categories, those who received their board certification before or during 1990 and those who received their board certification after 1990. Among those who responded to the question on year of board certification, a total of 95 physicians received board certification before or during 1990 and 83 physicians received board certification after the year 1990.

The distribution of practice site among the respondents was as follows: $35.1 \%$ were solo office-based, $33.6 \%$ were group office-based, $14.7 \%$ were hospital-based, $10.0 \%$ were university-affiliated hospital based, and $6.6 \%$ reported other practice sites. Among those who reported the primary practice site as hospital, 22 described it as rural, 9 described it as sub-urban and 12 described it as urban. Also, 6 worked in governmental/nonfederal settings, 12 worked in government/federal settings, 24 worked in private-not-for-profit settings, and 3 worked in private investor owned settings. A total of 23 respondents reported provision of tertiary care at their hospital and the average number of beds in the hospital was 266.56. The average number of new AMI patients treated by the respondents was 3.36 and the average number of repeat AMI patients treated was 9.64. 
Table 31: Demographic and Practice Characteristics of Respondents

Characteristics

Age

Less than 40 years

Average Age \pm S.D.

Gender

Males

Females

Specialty

Cardiology

Internal Medicine

Family Practice

General Practice

Others

Year of Board Certification

$\leq 1990$

$>1990$

Primary Practice Site

Solo, office-based

Group, office-based
57

73

80

44

$49.23 \pm 10.96$

214

45

95

83

Percent

82.6

17.4

9.7

32.6

47.7

6.6

46.6

87 
Table 31: Demographic and Practice Characteristics of Respondents (contd.)

\begin{tabular}{|c|c|c|}
\hline Characteristics & $\mathbf{N}$ & Percent \\
\hline Hospital based & 38 & 14.7 \\
\hline University-affiliated Hospital based & 26 & 10.0 \\
\hline Others & 17 & 6.6 \\
\hline \multicolumn{3}{|l|}{ Hospital Characteristics } \\
\hline Rural & 22 & 31.4 \\
\hline Sub-urban & 9 & 12.9 \\
\hline Urban & 12 & 17.1 \\
\hline Government/Non-federal & 6 & 8.6 \\
\hline Government/Federal & 12 & 17.1 \\
\hline Private Not-for-Profit & 24 & 34.3 \\
\hline Private Investor Owned & 3 & 4.3 \\
\hline Provides Tertiary Care & 23 & 32.9 \\
\hline Number of beds Mean (S.D.), Min-Max & $266.56(208.40)$ & $18-900$ \\
\hline Number of new AMI patients Mean (S.D.), Min-Max & $3.36(5.40)$ & $0-50$ \\
\hline Number of repeat AMI patients Mean (S.D.), Min-Max & $9.64(33.92)$ & $0-450$ \\
\hline
\end{tabular}

N: Number of physicians, S.D: Standard deviation, AMI: Acute myocardial infarction, Min: Minimum value, Max: Maximum value 


\section{Results for Research Question 5}

The guidelines classify three conditions as absolute contraindications:

hypotension, bradycardia and atrioventricular (AV) block. The guidelines also classify second and third degree AV block as relative contraindications. Hence, there is uncertainty whether AV block is an absolute contraindication or a relative contraindication. For study purposes, first degree AV block was considered to be a relative contraindication and second and third degree AV blocks were considered to be absolute contraindications.

Hence, four absolute contraindications used in the questionnaire were hypotension, bradycardia, second degree and third degree AV blocks (Table 32). About $35 \%$ of the respondents classified hypotension as an absolute contraindication and almost $61 \%$ classified bradycardia as an absolute contraindication. Exactly $50 \%$ of the respondents classified second degree AV block and greater than $85 \%$ classified third degree AV block as absolute contraindications.

The relative contraindications in the questionnaire were systolic blood pressure greater than $100 \mathrm{Hg}$, heart rate less than $60 \mathrm{bpm}$, peripheral vascular disease, peripheral hypoperfusion, $\mathrm{EF}<40 \%$, first degree AV block, COPD, asthma, insulin dependent diabetes mellitus (IDDM), PR-interval greater than 0.24 second, and beta-blocker intolerance. A high percent of respondents classified systolic blood pressure $<100 \mathrm{hg}$ (about 78\%), heart rate<60bpm(75\%), COPD (about 83\%) and asthma (about 76\%) as relative contraindications. Greater than $50 \%$ of the respondents classified peripheral vascular disease (58\%), peripheral hypoperfusion (55\%), first degree AV block (53\%), IDDM (about 60\%) and PR>0.24 second (about 64\%) as relative contraindications. A 
relatively lower percent of respondents classified $\mathrm{EF}<40 \%$ (34\%) and beta-blocker intolerance $(41 \%)$ as a relative contraindication.

There were four conditions in the questionnaire, which were neither absolute nor relative contraindications for beta-blockers. These include hypertension, non-insulin diabetes mellitus (NIDDM), stroke and ACE-inhibitor intolerance. A very high percentage of respondents classified three of these conditions - hypertension (98\%), stroke (about 88\%), and ACE-inhibitor intolerance (about 91\%) as not a contraindication for beta-blocker therapy. However, NIDDM was classified almost equally as a relative contraindication (49\%) and not a contraindication (50\%).

Three aggregate scores were computed based on correct classification of each of the conditions in the questionnaire in the three groups - absolute contraindication, relative contraindication, and not a contraindication (Table 33). The mean absolute contraindication score was $2.29(\mathrm{SD}=1.20)$ out of a total of 4 responses. The mean relative contraindication score was $6.73(\mathrm{SD}=2.10)$ out of a total of 11 correct responses. And the mean not a contraindication score was $3.17(\mathrm{SD}=0.84)$ out of a total of 4 responses.

The number of patients prescribed beta-blockers by the respondent among the last 10 was obtained from the survey question 3, in Section A. Prescribing percent was calculated by dividing the response with 10 and multiplying it by 100 . Thus, the mean number of patients prescribed beta-blocker among the last 10 individuals treated by the physician was $9.01(\mathrm{SD}=1.35)$ and the mean prescribing percent of beta-blockers was $90.10(\mathrm{SD}=13.5)$. This prescribing percent was a proxy for prescribing behavior. 
A correlation matrix was developed for the prescribing percent/behavior with absolute contraindication score, relative contraindication score and not a contraindication score (Table 34). The only correlation that was significant was the not a contraindication score and it was positively related to prescribing percent. Thus, those respondents who classified the conditions which were not contraindications correctly, were more likely to prescribe beta-blockers among the post-AMI patients. 
Table 32: Knowledge of Contraindications

\begin{tabular}{|c|c|c|c|}
\hline Patient conditions & $\begin{array}{l}\text { Absolute } \\
\text { contraindication }\end{array}$ & $\begin{array}{l}\text { Relative } \\
\text { contraindication }\end{array}$ & $\begin{array}{l}\text { Not a } \\
\text { contraindication }\end{array}$ \\
\hline & $\%$ & $\%$ & $\%$ \\
\hline
\end{tabular}

\section{Absolute contraindications}

Hypotension (systolic pressure <90hg)

Bradycardia (heart 158

$61.00 \quad 98$

37.8

rate $<50 \mathrm{bpm}$ )

Second degree AV block

129

50.00

119

$46.12 \quad 10$

3.88

Third degree AV block

220

$85.27 \quad 29$

$11.24 \quad 9$

\section{Relative}

contraindications

Systolic blood pressure 13

$<100 \mathrm{hg}$

Heart rate $<60 \mathrm{bpm}$

15

5.79

196

$75.68 \quad 48$

18.53

Peripheral vascular

7

2.71

150

$58.14 \quad 101$

39.15

disease

Peripheral hypoperfusion $\quad 56$

$22.22 \quad 139$

$55.16 \quad 57$

22.62

$\mathrm{EF}<40 \%$

13

5.06

88

$34.24 \quad 156$

60.70

First degree AV block

15

$5.84 \quad 138$

53.70

104

40.47

COPD

10

3.85

217

83.46

33

12.69

Asthma

49

18.80

197

$75.50 \quad 15$

5.70

IDDM

14

5.43

156

$60.47 \quad 88$

34.11 
Table 32: Knowledge of Contraindications (contd.)

\begin{tabular}{|c|c|c|c|c|c|c|}
\hline \multirow[t]{2}{*}{ Patient conditions } & \multicolumn{2}{|c|}{$\begin{array}{l}\text { Absolute } \\
\text { contraindication }\end{array}$} & \multicolumn{2}{|c|}{$\begin{array}{l}\text { Relative } \\
\text { contraindication }\end{array}$} & \multicolumn{2}{|c|}{$\begin{array}{l}\text { Not a } \\
\text { contraindication }\end{array}$} \\
\hline & $\mathbf{N}$ & $\%$ & $\mathbf{N}$ & $\%$ & $\mathbf{N}$ & $\%$ \\
\hline PR $>0.24$ second & 38 & 15.02 & 165 & 65.22 & 50 & 19.76 \\
\hline Beta-blocker intolerance & 149 & 57.53 & 108 & 41.70 & 2 & 0.77 \\
\hline \multicolumn{7}{|l|}{$\underline{\text { Not a contraindication }}$} \\
\hline $\begin{array}{l}\text { Hypertension (systolic } \\
\text { pressure }>140 \mathrm{hg} \text { ) }\end{array}$ & 0 & 0 & 6 & 2.31 & 254 & 97.69 \\
\hline NIDDM & 3 & 1.16 & 126 & 48.65 & 130 & 50.19 \\
\hline Stroke & 1 & 0.04 & 31 & 12.11 & 224 & 87.50 \\
\hline $\begin{array}{l}\text { ACE-inhibitor } \\
\text { Intolerance }\end{array}$ & 8 & 3.29 & 15 & 6.17 & 220 & 90.53 \\
\hline
\end{tabular}

$\mathrm{N}$ : Number of physicians, AV: Atrioventricular, EF: Ejection fraction, COPD: Chronic obstructive pulmonary disease, IDDM: Insulin dependent diabetes mellitus, NIDDM: Non insulin dependent diabetes mellitus, ACE: Angiotensin converting enzyme 
Table 33: Knowledge Scores

\begin{tabular}{lcc} 
Variables & Mean \pm S.D. & Range \\
\hline Absolute contraindication score & $2.29 \pm 1.20$ & $0-4$ \\
Relative contraindication score & $6.73 \pm 2.10$ & $0-11$ \\
Not a contraindication score & $3.17 \pm 0.84$ & $0-4$ \\
$\begin{array}{l}\text { Number of patients prescribed beta- } \\
\text { blockers }\end{array}$ & $9.01 \pm 1.35$ & $2-10$ \\
Prescribing percent/behavior & $90.10 \pm 13.50$ & $20-100$ \\
\hline
\end{tabular}

S.D: Standard deviation 
Table 34: Correlation Matrix for Knowledge of Contraindications

\begin{tabular}{lllll}
\hline Variables & $\begin{array}{l}\text { Prescribing } \\
\text { percent/ } \\
\text { behavior }\end{array}$ & $\begin{array}{l}\text { Absolute } \\
\text { contraindica- } \\
\text { tion score }\end{array}$ & $\begin{array}{l}\text { Relative } \\
\text { contraindica- } \\
\text { tion score }\end{array}$ & $\begin{array}{l}\text { Not a } \\
\text { contraindica- } \\
\text { tion score }\end{array}$ \\
\hline $\begin{array}{l}\text { Prescribing } \\
\text { percent/ behavior }\end{array}$ & 1.000 & 1.000 & \\
$\begin{array}{l}\text { Absolute } \\
\text { contraindication }\end{array}$ & -0.032 & & & \\
score & & 0.114 & 1.000 & \\
$\begin{array}{l}\text { Relative } \\
\text { contraindication } \\
\text { score }\end{array}$ & 0.050 & & & \\
$\begin{array}{l}\text { Not a } \\
\text { contraindication } \\
\text { score }\end{array}$ & $0.229 *$ & -0.053 & -0.098 & \\
\hline
\end{tabular}

* Significant at $\mathrm{p} \leq 0.05$ 


\section{Discussion for Research Question 5}

Greater than $50 \%$ of the respondents classified three of the four listed absolute contraindications correctly. The one condition that had a lower percent of physicians classifying it as a contraindication was hypotension. Similarly, except for beta-blocker intolerance and $\mathrm{EF}<40 \%$ all the other relative contraindications were also classified correctly by greater than $50 \%$ of the physicians. Of the 4 conditions, which were neither absolute nor relative contraindications, three- hypertension, stroke and ACE-inhibitor intolerance were classified correctly as not a contraindication by greater than $85 \%$ of the physicians. However, a relatively high percent of physicians classified NIDDM as a relative contraindication. Thus, there appears to be higher than average awareness about the relative and absolute contraindications for beta-blocker therapy in post AMI patients. Based on the correct classification of these conditions three scores were computed, the absolute contraindication score, the relative contraindication sore and the not a contraindication score. Of these, two scores - the absolute contraindication score and the relative contraindication score were not significantly correlated with prescribing percent. Thus, the null hypothesis $\mathrm{F}$ that there is no association between knowledge of absolute and relative contraindications and prescribing behavior is not rejected. The not a contraindication score was significantly correlated with prescribing percent. Thus, correct knowledge of conditions that are not contraindications for use does result in positive prescribing behavior.

\section{$\underline{\text { Results for Research Question } 6}$}

The questionnaire asked the respondents to give their willingness to prescribe beta-blockers in patients with different conditions on a Likert scale from 1 to 5 where 1 
was least likely and 5 was most likely. These conditions could be divided into three categories: those conditions where beta-blockers definitely provide benefits, hence therapy should be definitely prescribed to the patient; those conditions where therapy may be prescribed as there is uncertainty about benefits to the patient either because of lower risk or because of presence of relative contraindications; and those conditions in the presence of which beta-blockers should definitely not be prescribed as these are absolute contraindications and in the presence of these conditions beta-blockers do not provide benefit and may be harmful.

The conditions in the definitely prescribe category were: age $>50$ years $($ Mean $=$ 4.76), large or anterior AMI (Mean = 4.55), previous AMI (Mean =4.77), patient who had an angioplasty (Mean $=4.64)$, or by-pass surgery $($ Mean $=4.67)$, patients with stroke $($ Mean $=4.23)$, hypertension $($ Mean $=4.84)$ and complex ventricular ectopy $($ Mean $=$ 4.19) (Table 35). The conditions in the maybe prescribe category were: age $\leq 50$ years $($ Mean $=4.74)$, small infarction $($ Mean $=4.75), \mathrm{EF}<40 \%($ Mean $=4.16)$, history of CHF $($ Mean $=3.95)$, systolic blood pressure $<100 \mathrm{Hg}($ Mean $=2.97)$, heart rate $<60 \mathrm{bpm}($ Mean $=$ 2.75), LV failure $($ Mean $=3.76)$, first degree AV block $($ Mean $=3.20)$, peripheral vascular disease $($ Mean $=3.26)$, COPD $($ Mean $=2.89)$, asthma $($ Mean $=2.36)$, and IDDM $($ Mean $=3.58)$. The conditions in the definitely not prescribe category were: hypotension $($ Mean $=2.30)$, bradycardia $($ Mean $=1.72)$, second degree AV block $($ Mean $=1.69)$ and third degree AV block $($ Mean $=1.31)$.

A score was computed for each of the three categories by adding the individual answers for each condition within the category (Table 36). Thus, the definitely prescribe score had a mean of $36.75(\mathrm{SD}=4.28)$ and ranged from 8 to 40 . The maybe prescribe 
score had a mean of $42.36(\mathrm{SD}=8.26)$ and ranged from 17 to 60 . And the mean for definitely not prescribe score was $7.01(\mathrm{SD}=2.91)$ and ranged from 2.5 to 18 . The mean for prescribing percent/behavior was $90.10(\mathrm{SD}=13.50)$.

A correlation matrix was developed for the prescribing percent with definitely prescribe score, maybe prescribe score and the definitely not prescribe score (Table 37). All the three scores had significant correlations with prescribing percent. The correlation coefficient was approximately 0.47 for definitely prescribe score, and 0.41 for maybe prescribe score, and was 0.13 for the definitely not prescribe score. Thus, those with higher scores in all the three categories were more likely to prescribe beta-blockers among the post-AMI patients. 
Table 35: Willingness to Prescribe Beta-blockers

$\begin{array}{lll}\text { Patient Characteristics } & \text { Mean } & \text { S.D. }\end{array}$

\section{Definitely prescribe}

Age $>50$ years

Large or anterior MI

Previous infarction

Angioplasty

By-pass surgery

Stroke

Hypertension

Complex ventricular ectopy

Maybe prescribe

Age $\leq 50$ years

Small infarction

$\mathrm{EF}<40 \%$

History of CHF

Systolic blood pressure $<100 \mathrm{Hg}$

Heart rate $<60 \mathrm{bpm}$

LV failure

First degree AV block

Peripheral vascular disease

COPD

Asthma
4.76

0.56

4.55

0.87

4.77

0.66

4.64

0.80

4.67

0.75

4.23

1.07

4.84

0.53

4.19

1.17

4.74

0.63

4.75

0.58

4.16

1.06

3.95

1.13

2.97

1.27

2.75

1.28

3.76

1.21

3.20

1.36

3.26

1.38

2.89

1.20

2.36

1.17 
Table 35: Willingness to Prescribe Beta-blockers (contd.)

\begin{tabular}{lll}
\hline Patient Characteristics & Mean & S.D. \\
\hline IDDM & 3.58 & 1.25 \\
Definitely not prescribe & & \\
Hypotension & 2.30 & 1.18 \\
Bradycardia & 1.72 & 0.99 \\
Second degree AV block & 1.69 & 1.05 \\
Third degree AV block & 1.31 & 0.92 \\
\hline
\end{tabular}

S.D.: Standard deviation, MI: Myocardial infarction, EF: Ejection fraction, CHF: Congestive heart failure, LV: Left ventricular, AV: Atrioventricular, COPD: Chronic obstructive pulmonary disease, IDDM: Insulin dependent diabetes mellitus 
Table 36: Willingness to Prescribe Scores

\begin{tabular}{lcc} 
Variables & Mean \pm S.D. & Range \\
\hline $\begin{array}{lc}\text { Definitely prescribe score } \\
\text { May be prescribe score }\end{array}$ & $36.65 \pm 4.28$ & $8-40$ \\
$\begin{array}{l}\text { Definitely not prescribe score } \\
\text { Number of patients prescribed beta- } \\
\text { blockers }\end{array}$ & $42.36 \pm 8.26$ & $17-60$ \\
$\begin{array}{l}\text { Prescribing percent/ behavior } \\
\text { P. }\end{array}$ & $9.01 \pm 1.35$ & $2.5-18$ \\
\hline
\end{tabular}

S.D: Standard deviation 
Table 37: Correlation Matrix for Willingness to Prescribe

\begin{tabular}{lllll}
\hline Variables & $\begin{array}{l}\text { Prescribing } \\
\text { percent/ } \\
\text { behavior }\end{array}$ & $\begin{array}{l}\text { Definitely } \\
\text { prescribe score }\end{array}$ & $\begin{array}{l}\text { Maybe } \\
\text { prescribe score }\end{array}$ & $\begin{array}{l}\text { Definitely not } \\
\text { prescribe } \\
\text { score }\end{array}$ \\
\hline $\begin{array}{l}\text { Prescribing } \\
\text { percent/ } \\
\text { behavior }\end{array}$ & 1.00 & & & \\
$\begin{array}{l}\text { Definitely } \\
\text { prescribe score }\end{array}$ & $0.474^{*}$ & 1.00 & & \\
$\begin{array}{l}\text { May be } \\
\text { prescribe score }\end{array}$ & $0.410^{*}$ & $0.648^{*}$ & 1.00 & \\
$\begin{array}{l}\text { Definitely not } \\
\text { prescribe score }\end{array}$ & $0.134^{*}$ & $0.194^{*}$ & & 1.00 \\
\hline
\end{tabular}

* Significant at $\mathrm{p} \leq 0.05$ 


\section{Discussion for Research Question 6}

The willingness to prescribe beta-blockers as post-AMI medication was high (means greater than 4 out of 5) for those conditions where beta-blockers should definitely be prescribed for secondary prevention. For those conditions where beta-blocker maybe prescribed as secondary prevention agents, the willingness to prescribe means were between 2 and 5. In addition, the means for willingness to prescribe beta-blockers as secondary preventive agents post-AMI in patients with conditions where it should definitely not be prescribed were between 1 and 3 . Thus, this indicates that willingness to prescribe is evidence-based and physicians definitely are aware of the guidelines. When the correlations of the three scores were calculated with prescribing percent, all three were significant. Thus, reject null hypothesis $\mathrm{G}$ that there is no relationship between physicians' willingness to prescribe beta-blockers as post AMI secondary preventive agents and physicians' prescribing of these agents is rejected. The analysis revealed a positive relationship between willingness to prescribe and prescribing behavior among physicians for beta-blockers post-AMI irrespective of patient co-morbidities.

\section{Results for Research Question 7}

To assess the effects of demographic and practice characteristics on prescribing behavior, t-tests, ANOVA's (Table 38) and correlations (Table 39) were conducted. Gender and year of board certification was investigated using t-tests whereas, specialty, location and practice site were investigated using F-tests. Association of age, size of hospital, number of new AMI patients, and number of repeat AMI patients treated were determined through Pearson's correlations. 
Age and size of hospital were significantly correlated with prescribing percent. Age was negatively associated with prescribing percent/behavior. Thus, older physicians were less likely to prescribe beta-blockers in post-AMI patients. Size of hospital was positively associated with prescribing percent. Thus, larger the size of the hospital the physicians worked in, the more likely they were to prescribe beta-blockers in post-AMI patients. Prescribing percent/behavior was not significantly different by gender, specialty, location, practice site and year of board certification. Prescribing percent/behavior was higher for cardiologists and physicians from internal medicine compared to family practitioners and general practitioners, also physicians from urban areas had higher prescribing percent compared to rural and sub-urban areas, females had a higher prescribing percent compared to males, and finally physicians who were boardcertified after 1990 had higher prescribing percent compared to those who were board certified before or during 1990, however, none of these were statistically significant. 
Table 38: Prescribing Behavior and Demographic Characteristics, Part 1

\begin{tabular}{|c|c|c|c|}
\hline Variables & $\begin{array}{l}\text { Prescribing percent/ } \\
\text { behavior } \\
\text { (Mean } \pm \text { S.D.) }\end{array}$ & Statistic & Significance \\
\hline Gender & & $\mathrm{t}=-1.15$ & 0.252 \\
\hline Males & $89.60 \pm 14.21$ & & \\
\hline Females & $92.21 \pm 9.41$ & & \\
\hline Specialty & & $\mathrm{F}=1.73$ & 0.143 \\
\hline Cardiology & $90.40 \pm 13.38$ & & \\
\hline Internal medicine & $92.88 \pm 9.44$ & & \\
\hline Family practice & $88.29 \pm 15.11$ & & \\
\hline General practice & $84.29 \pm 20.70$ & & \\
\hline Other & $90.83 \pm 13.79$ & & \\
\hline Location & & $F=0.363$ & 0.699 \\
\hline Rural & $92.63 \pm 14.47$ & & \\
\hline Sub-urban & $90.00 \pm 9.26$ & & \\
\hline Urban & $95.00 \pm 9.72$ & & \\
\hline Practice site & & $\mathrm{F}=1.41$ & 0.230 \\
\hline Solo, office based & $87.73 \pm 16.24$ & & \\
\hline Group, office-based & $90.18 \pm 12.48$ & & \\
\hline Hospital-based & $93.43 \pm 11.87$ & & \\
\hline $\begin{array}{l}\text { University-affiliated } \\
\text { hospital based }\end{array}$ & $92.50 \pm 8.47$ & & \\
\hline Other & $90.77 \pm 9.54$ & & \\
\hline
\end{tabular}


Table 38: Prescribing Behavior and Demographic Characteristics, Part 1 (contd.)

\begin{tabular}{clll}
\hline Variables & $\begin{array}{l}\text { Prescribing percent/ } \\
\text { behavior } \\
\text { (Mean } \pm \text { S.D. })\end{array}$ & Statistic & \\
\hline Year of board certification & $\mathrm{t}=-1.704$ & 0.090 \\
$\leq 1990$ & $88.52 \pm 14.15$ & & \\
$>1990$ & $91.79 \pm 10.16$ & & \\
& & \\
\hline
\end{tabular}

S.D: Standard deviation 
Table 39: Prescribing Behavior and Demographic Characteristics, Part 2

\begin{tabular}{llllll} 
Variables & $\begin{array}{l}\text { Prescribing } \\
\text { percent/ } \\
\text { behavior }\end{array}$ & Age & $\begin{array}{l}\text { Size of } \\
\text { hospital }\end{array}$ & $\begin{array}{l}\text { Number of } \\
\text { new AMI } \\
\text { patients }\end{array}$ & $\begin{array}{l}\text { Number of } \\
\text { repeat AMI } \\
\text { patients }\end{array}$ \\
\hline $\begin{array}{l}\text { Prescribing } \\
\text { percent/ } \\
\text { behavior }\end{array}$ & 1.00 & & & \\
Age & $-0.199^{*}$ & 1.00 & & \\
$\begin{array}{l}\text { Size of } \\
\text { hospital }\end{array}$ & $0.231^{*}$ & -0.092 & 1.00 & & \\
$\begin{array}{l}\text { Number of } \\
\text { new AMI } \\
\text { patients }\end{array}$ & 0.061 & -0.074 & $0.184^{*}$ & 1.00 & \\
$\begin{array}{l}\text { Number of } \\
\text { repeat AMI } \\
\text { patients }\end{array}$ & 0.047 & & & & \\
\hline
\end{tabular}

* Significant at $\mathrm{p} \leq 0.05$, AMI: Acute myocardial infarction 


\section{Discussion for Research Question 7}

Based on the analysis, the null hypotheses $\mathrm{H}$ that there is no relationship between demographic and practice characteristics and prescribing behavior is rejected. Age and size of the hospital do have an association with prescribing behavior. Younger physicians and physicians who worked in larger hospitals had higher prescribing percentages. Nicholls et al., (2001) reported higher prescribing rates in a large hospital compared to a small hospital. However, the large hospital in this study was also affiliated to a university and cardiologists treated patients in the large hospital. Thus, it is possible that when other characteristics of the hospital are adjusted for, size of hospital may not have an impact on prescribing behavior. Also, age has been reported to be associated with prescribing behavior in other disease conditions such as hypertension (Mehta et al., 1999). Younger physicians were more likely to prescribe recommended agents compared to older physicians. Since beta-blockers are recommended medications post-AMI, this study establishes a similar association between age and prescribing behavior for betablockers too. This study did not find an association between prescribing behavior and other physician characteristics contrary to results of previous studies which have reported that specialty, year of board certification, location, and university affiliation do affect prescribing behavior of physicians for beta-blockers as secondary preventive agents post AMI (Ayanian et al. 1994; Fehrenbach et al, 2001; Sheikh \& Bullock, 2001).

\section{Results for Research Question 8}

Similar to the analyses in research question 7, t-tests, ANOVA's and Pearson's correlations were conducted on the demographic and practice characteristics with the 
three knowledge scores and the three willingness to prescribe scores. The results for the three knowledge scores are reported in Tables 40 and 41 and the results for the three willingness to prescribe scores are reported in Tables 42 and 43.

Thus, we see that the knowledge scores did not differ by gender, location and year of board certification. However, the absolute contraindication score and the not a contraindication score were significantly different by specialty, and the not a contraindication score was also significantly different by primary practice site. The general practitioners had a higher absolute contraindication score compared to physicians from internal medicine. Thus, the general practitioners classified the absolute contraindications more correctly than physicians in internal medicine. The cardiologists had a higher not a contraindication score compared to family practitioners, which implies that cardiologists classified those conditions which were not contraindications more correctly compared to family practitioners. The not a contraindication score was also significantly different by primary practice site. Those who practiced in universityaffiliated hospital settings classified those conditions which were not contraindications for beta-blocker therapy more correctly compared those who practices in solo, officebased settings. The not a contraindication score was also negatively correlated to age. Thus, older physicians were less likely to classify those conditions which were not contraindications for beta-blocker therapy correctly. Similarly, the number of repeat AMI patients treated was also negatively correlated to absolute contraindication score. Thus, those physicians who treated more repeat AMI patients were less likely to classify those conditions which were absolute contraindications for beta-blocker therapy correctly. 
Willingness to prescribe scores (Tables $42 \& 43$ ) were also analyzed in a similar manner. These scores were not different by gender, location, and year of board certification. Specialty was significantly different for the definitely prescribe score and the maybe prescribe score. The general practitioners had a lower definitely prescribe score compared to internal medicine physicians. Thus, physicians in general practice were less willing to prescribe beta-blockers as post-AMI medication to those patients in whom it should definitely be prescribed when compared to physicians from internal medicine. A similar relation was found for the maybe prescribe score, with the general practitioners' and family practitioners' scores being significantly lower than physicians' in internal medicine. Thus, physicians from general practice and family practice were less willing to prescribe beta-blockers as post AMI medications in patients with those conditions where they maybe prescribed when compared to physicians in internal medicine.

Both 'definitely prescribe' score and 'maybe prescribe' score were significantly different by primary practice site. Physicians in solo office-based practice had a significantly lower definitely prescribe score compared to physicians at universityaffiliated hospitals. Also, physicians in solo practice had a significantly lower maybe prescribe score compared to both physicians from hospital practice and physicians from university-affiliated hospitals.

Age was significantly and negatively correlated to two scores, definitely prescribe, and maybe prescribe. Thus, older physicians were less willing to prescribe beta-blockers as post AMI medication in patients with conditions where they should be definitely prescribed, and maybe prescribed. Size of hospital was positively associated 
with definitely prescribe and maybe prescribe scores. Thus, physicians from larger hospitals were more willing to prescribe beta-blockers in patients where they should definitely or maybe be prescribed. Number of new AMI patients and number of repeat AMI patients treated by the physicians were not related to the willingness to prescribe scores. 
Table 40: Knowledge of Contraindications and Demographic Characteristics, Part 1

\begin{tabular}{llll}
\hline Variables & $\begin{array}{l}\text { Absolute } \\
\text { contraindication } \\
\text { score }\end{array}$ & $\begin{array}{l}\text { Relative } \\
\text { contraindication } \\
\text { score }\end{array}$ & $\begin{array}{l}\text { Not a } \\
\text { contraindication } \\
\text { score }\end{array}$ \\
\hline
\end{tabular}

Gender

$\begin{array}{llll}\text { Males } & 2.29 \pm 1.19 & 6.74 \pm 2.14 & 3.17 \pm 0.88 \\ \text { Females } & 2.27 \pm 1.21 & 6.64 \pm 1.87 & 3.22 \pm 0.67\end{array}$

Specialty

$\begin{array}{llll}\text { Cardiology } & 2.28 \pm 0.98 & 6.04 \pm 2.09 & 3.60 \pm 0.58^{\mathrm{a}} \\ \text { Internal medicine } & 2.01 \pm 1.14^{\mathrm{a}} & 6.80 \pm 2.03 & 3.24 \pm 0.82 \\ \text { Family practice } & 2.33 \pm 1.24 & 6.75 \pm 2.09 & 3.08 \pm 0.88^{\mathrm{a}} \\ \text { General practice } & 3.22 \pm 0.97^{\mathrm{a}} & 5.89 \pm 2.98 & 2.78 \pm 1.20 \\ \text { Other } & 2.76 \pm 1.25 & 7.41 \pm 1.66 & 3.18 \pm 0.64\end{array}$

Location

$\begin{array}{llll}\text { Rural } & 2.38 \pm 1.36 & 6.71 \pm 1.79 & 3.10 \pm 0.70 \\ \text { Sub-urban } & 2.44 \pm 1.13 & 6.22 \pm 2.22 & 3.44 \pm 0.53 \\ \text { Urban } & 2.18 \pm 1.40 & 6.36 \pm 1.57 & 3.45 \pm 0.69\end{array}$

Practice site

$\begin{array}{llll}\text { Solo, office based } & 2.21 \pm 1.25 & 6.42 \pm 2.20 & 2.96 \pm 1.03^{\mathrm{a}} \\ \text { Group, office-based } & 2.32 \pm 1.16 & 6.87 \pm 1.97 & 3.28 \pm 0.69 \\ \text { Hospital-based } & 2.29 \pm 1.39 & 6.87 \pm 2.17 & 3.34 \pm 0.62 \\ \begin{array}{l}\text { University-affiliated } \\ \text { hospital based }\end{array} & 2.35 \pm 0.94 & 6.88 \pm 2.08 & 3.46 \pm 0.70^{\mathrm{a}} \\ \text { Other } & & & \\ & 2.35 \pm 1.11 & 7.00 \pm 2.12 & 2.88 \pm 0.78\end{array}$


Table 40: Knowledge of Contraindications and Demographic Characteristics, Part 1 (contd.)

\begin{tabular}{clll}
\hline Variables & $\begin{array}{l}\text { Absolute } \\
\text { contraindication } \\
\text { score }\end{array}$ & $\begin{array}{l}\text { Relative } \\
\text { contraindication } \\
\text { score }\end{array}$ & $\begin{array}{l}\text { Not a } \\
\text { contraindication } \\
\text { score }\end{array}$ \\
\hline $\begin{array}{l}\text { Year of board } \\
\text { certification }\end{array}$ & & & \\
$\leq 1990$ & $2.24 \pm 1.46$ & $6.45 \pm 2.19$ & $3.17 \pm 0.83$ \\
$>1990$ & $2.35 \pm 1.11$ & $6.94 \pm 1.91$ & $3.33 \pm 0.68$ \\
\hline
\end{tabular}

t-tests: gender and year of board certification

ANOVA's: specialty, location, and practice site. Significant ANOVA's were investigated with post-hoc Tukey comparisons

a: Significant at $\mathrm{p} \leq 0.05$ 
Table 41: Knowledge of Contraindications and Demographic Characteristics, Part 2

$\begin{array}{rlllllll}\text { Variables } & \begin{array}{l}\text { Absolute } \\ \text { contrain- } \\ \text { dication } \\ \text { score }\end{array} & \begin{array}{l}\text { Relative } \\ \text { contrain- } \\ \text { dication } \\ \text { score }\end{array} & \begin{array}{l}\text { Not a } \\ \text { contrain- } \\ \text { dication }\end{array} & \text { Age } & \begin{array}{l}\text { Size of } \\ \text { hospital }\end{array} & \begin{array}{l}\text { Number } \\ \text { of new }\end{array} & \begin{array}{l}\text { Number } \\ \text { of } \\ \text { AMI }\end{array} \\ \text { repeat } \\ \text { patients } & \begin{array}{l}\text { AMI } \\ \text { patients }\end{array}\end{array}$

\begin{tabular}{|c|c|}
\hline $\begin{array}{l}\text { Absolute } \\
\text { contra- } \\
\text { indication } \\
\text { score }\end{array}$ & 1.000 \\
\hline $\begin{array}{l}\text { Relative } \\
\text { contra- } \\
\text { indication } \\
\text { score }\end{array}$ & 0.114 \\
\hline
\end{tabular}

$\begin{array}{llll}\text { Not a } & -0.053 & 0.098 & 1.000\end{array}$

contra-

indication

score

Age $\quad 0.001 \quad-0.047 \quad-0.160 * \quad 1.000$

$\begin{array}{llllll}\text { Size of } & -0.053 & -0.059 & 0.069 & -0.092 & 1.000\end{array}$

hospital

$\begin{array}{lllllll}\text { Number } & -0.027 & -0.060 & -0.009 & -0.074 & 0.184 * & 1.000\end{array}$

of new

AMI

patients

$\begin{array}{llllllll}\text { Number } & -0.156^{*} & -0.026 & 0.007 & 0.130 & 0.010 & 0.197 * & 1.000\end{array}$

of repeat

AMI

patients

\footnotetext{
* Significant at $\mathrm{p} \leq 0.05$, AMI: Acute myocardial infarction
} 
Table 42: Willingness to Prescribe and Demographic Characteristics, Part 1

Variables

Definitely prescribe score
Maybe prescribe score
Definitely not prescribe score

Gender

Males

Females

Specialty

Cardiology

Internal medicine

Family practice

General practice

Other

Location

Rural

Sub-urban

Urban
$36.68+4.43$

$36.81 \pm 3.27$

$37.16 \pm 6.36$

$37.45 \pm 3.22^{\mathrm{a}}$

$36.19+4.34$

$33.33 \pm 4.30^{\mathrm{a}}$

$37.97+3.06$

$37.48+3.44$

$34.22 \pm 12.89$

$37.82 \pm 3.19$

Practice site

Solo, office based

$35.59 \pm 4.79^{\mathrm{a}}$

$36.92+4.57$

$37.68+2.82$

$38.19 \pm 2.38^{a}$
$36.18+3.83$
University-affiliated hospital based

Other

Group, office-based

Hospital-based

Other

(n)

$42.32+8.55$

$7.00 \pm 2.94$

$42.52 \pm 6.64$

$7.10 \pm 2.86$ 
Table 42: Willingness to Prescribe and Demographic Characteristics, Part 1 (contd.)

\begin{tabular}{clll}
\hline Variables & $\begin{array}{l}\text { Definitely } \\
\text { prescribe score }\end{array}$ & $\begin{array}{l}\text { Maybe prescribe } \\
\text { score }\end{array}$ & $\begin{array}{l}\text { Definitely not } \\
\text { prescribe score }\end{array}$ \\
\hline $\begin{array}{l}\text { Year of board } \\
\text { certification } \\
\leq 1990\end{array}$ & $36.34 \pm 4.83$ & $42.64 \pm 8.29$ & $6.79 \pm 2.62$ \\
$>1990$ & $37.36 \pm 3.02$ & $43.05 \pm 7.50$ & $6.97 \pm 2.99$ \\
\hline
\end{tabular}

t-tests: gender and year of board certification

ANOVA's: specialty, location, and practice site. Significant ANOVA's were investigated with post-hoc Tukey comparisons

a,b: Significant at $\mathrm{p} \leq 0.05$ 
Table 43: Willingness to Prescribe and Demographic Characteristics, Part 2

\begin{tabular}{|c|c|c|c|c|c|c|c|}
\hline Variables & $\begin{array}{l}\text { Definitely } \\
\text { prescribe } \\
\text { score }\end{array}$ & $\begin{array}{l}\text { Maybe } \\
\text { prescribe } \\
\text { score }\end{array}$ & $\begin{array}{l}\text { Definitely } \\
\text { not } \\
\text { prescribe } \\
\text { score }\end{array}$ & Age & $\begin{array}{l}\text { Size of } \\
\text { hospital }\end{array}$ & $\begin{array}{l}\text { Number } \\
\text { of new } \\
\text { AMI } \\
\text { patients }\end{array}$ & $\begin{array}{l}\text { Number } \\
\text { of } \\
\text { repeat } \\
\text { AMI } \\
\text { patients }\end{array}$ \\
\hline $\begin{array}{l}\text { Definitely } \\
\text { prescribe } \\
\text { score }\end{array}$ & 1.000 & & & & & & \\
\hline $\begin{array}{l}\text { May be } \\
\text { prescribe } \\
\text { score }\end{array}$ & $0.648 *$ & 1.000 & & & & & \\
\hline $\begin{array}{l}\text { Definitely } \\
\text { not } \\
\text { prescribe } \\
\text { score }\end{array}$ & $0.194^{*}$ & $0.442 *$ & 1.000 & & & & \\
\hline Age & $-0.273^{*}$ & $-0.287 *$ & -0.091 & 1.000 & & & \\
\hline $\begin{array}{l}\text { Size of } \\
\text { hospital }\end{array}$ & $0.196^{*}$ & $0.193^{*}$ & 0.142 & -0.092 & 1.000 & & \\
\hline $\begin{array}{l}\text { Number } \\
\text { of new } \\
\text { AMI } \\
\text { patients }\end{array}$ & -0.002 & 0.085 & 0.028 & -0.074 & $0.184^{*}$ & 1.000 & \\
\hline $\begin{array}{l}\text { Number } \\
\text { of repeat } \\
\text { AMI } \\
\text { patients }\end{array}$ & -0.047 & 0.071 & 0.002 & 0.130 & 0.010 & $0.197 *$ & 1.000 \\
\hline
\end{tabular}

* Significant at $\mathrm{p} \leq 0.05$, AMI: Acute myocardial infarction 


\section{Discussion for Research Question 8}

Based on the results, both the null hypotheses I and $\mathrm{J}$ are rejected. The analysis revealed that specialty, type of practice site, age and number of repeat AMI patients treated were associated with knowledge scores. Similarly, specialty, type of practice site, age and size of the practice hospital were associated with willingness to prescribe scores.

The absolute contraindication score and the not a contraindication score were significantly different by specialty. Physicians from general practice had a higher absolute contraindication score compared to physicians from internal medicine and physicians from family practice had a lower not a contraindication score compared to cardiologists. Also, physicians from general practice had lower definitely prescribe score compared to physicians from internal medicine and physicians from both general and family practice had lower maybe prescribe score compared to physicians in internal medicine. Degree of specialization has been reported to be associated with knowledge of contraindications in other disease conditions like hypertension (Huse et al., 2001). An association between degree of specialization and knowledge was also reported by Sanchez et al. (2001) when they investigated cardiovascular diseases and Chlamydia pneumoniae. It makes intuitive sense that correct knowledge would result in higher willingness to prescribe scores, which in turn would eventually result in better prescribing behavior. Thus, though knowledge did not demonstrate a direct effect on prescribing behavior in research question 5, an indirect relationship may be present.

Type of practice site has not been reported to be associated with knowledge or willingness to prescribe before. On the other hand, type of primary practice site has been reported to be associated with prescribing behavior and this association usually involves 
presence of university or teaching affiliation, which results in higher prescribing rates of certain recommended medications such as aspirin (Venturini et al., 1999). This study revealed an association of university affiliation with both knowledge and willingness to prescribe scores. Thus, it is possible that university affiliation results in updated knowledge which increases willingness to prescribe and this may affect prescribing behavior.

Older physicians were less likely to identify those conditions that are not contraindications for beta-blockers correctly and were also less willing to prescribe betablockers in patients who should definitely receive them and those who may receive them. Previous reports have revealed that younger physicians are more likely to select medications consistent with guideline recommendations in other disease areas such as hypertension (Mehta et al., 1999). This study revealed an association between age and prescribing behavior, age and knowledge, and age and willingness to prescribe. Thus, this suggests that younger physicians are more aware of clinical practice guidelines, in other words are up to date with knowledge, which results in a higher willingness to prescribe which in turn results in their prescribing behavior being more consistent with such guidelines.

Among other variables, size of hospital was positively associated with two of the three ('definitely prescribe' and 'maybe prescribe') willingness to prescribe scores. It is possible that larger hospitals have better in-house seminars, and/or continuing medical education which help keep their physicians up to date about changes in guidelines and this results in a positive effect on their willingness to prescribe recommended therapies. 
Finally, number of repeat AMI patients was negatively associated with absolute contraindication score. It is possible that higher workload gives physicians less time to keep up with changes and this affects their knowledge.

\section{$\underline{\text { Results for Research Question } 9}$}

Effect of all physician characteristics such as demographic, practice, knowledge and willingness to prescribe on physician prescribing percent/behavior was investigated by regression analysis. The dependent variable was prescribing percent/behavior. The independent variables included; age, gender, specialty, practice site, absolute contraindication score, relative contraindication score, not a contraindication score, definitely prescribe score, maybe prescribe score, definitely not prescribe score, number of new AMI patients and number of repeat AMI patients treated by the physician per month. Specialty was converted to a dichotomous variable where 1 was cardiology and all the other specialties were grouped together as 0 , which implied non-cardiology or internal medicine, family practice, general practice or others. Similarly, practice site was converted into a dichotomous variable, where 1 implied university affiliation and 0 implied either solo or group office-based, non-university affiliated hospital or others. Location and size of hospital was not included in the analysis as only those physicians who worked in a hospital were asked to answer these questions. Year of board certification was not included in analysis as the data was missing for more than $33 \%$ of the respondents.

The resultant model was significant and had an $\mathrm{R}^{2}$ of $26.8 \%$, implying that the model explained almost $27 \%$ of the variance in prescribing percent/behavior (Table 44). 
The models were checked for violation of assumptions associated with linear regression. The model had no problems with multicollinearity, heteroskedasticity and autocorrelation. The analysis had a power of 95.6\%. However, the only independent variable that was a significant predictor of prescribing percent/behavior was the definitely prescribe score. Thus, physicians with higher willingness to prescribe beta-blockers in those patients where it should be definitely prescribed were more likely to have a higher prescribing percent for beta-blockers as secondary prevention agents post-AMI. 
Table 44: Predictors of Prescribing Percent/Behavior

Predictor

\begin{tabular}{lcccc}
\hline Age & -0.006 & 0.082 & -0.084 & 0.934 \\
Gender & -1.202 & 2.106 & -0.571 & 0.569 \\
Specialty- Cardiology & -0.518 & 2.905 & -0.179 & 0.859 \\
Practice site - University affiliation & -0.262 & 3.035 & -0.086 & 0.931 \\
Absolute contraindication score & 0.529 & 0.753 & 0.703 & 0.483 \\
Relative contraindication score & -0.107 & 0.383 & -0.281 & 0.779 \\
Not a contraindication score & -0.375 & 1.082 & -0.346 & 0.730 \\
Definitely prescribe score & 1.138 & 0.251 & 4.538 & $0.000 *$ \\
Maybe prescribe score & 0.233 & 0.148 & 1.577 & 0.116 \\
Definitely not prescribe score & 0.276 & 0.337 & 0.818 & 0.415 \\
Number of new AMI patients & 0.057 & 0.194 & 0.295 & 0.768 \\
Number of repeat AMI patients & 0.02 & 0.023 & 0.862 & 0.390 \\
\multicolumn{1}{c}{$\mathrm{R}^{2}=26.8 \%\left(\mathrm{~F}=5.908, \mathrm{p}<0.000^{*}\right), \mathrm{n}=206$} & \\
\hline
\end{tabular}

* Significant at $\mathrm{p} \leq 0.05$, S.E: Standard error, AMI: Acute myocardial infarction

Estimate S.E. t-Statistic Significance 


\section{Discussion for Research Question 9}

The null hypothesis $\mathrm{K}$ that there is no relationship between physicians prescribing of beta-blocker therapy post-AMI and the interaction of physician characteristics (demographic, practice, knowledge and willingness to prescribe) is rejected. Regression analysis which included physician knowledge scores, willingness to prescribe scores and demographic and practice characteristics helped in understanding how each of these traits affected prescribing behavior when the others were adjusted for. The results revealed that one of the willingness to prescribe scores explained a high percent of the variance in prescribing behavior for beta-blockers when it is used as a post-AMI medication when all the other characteristics were used as covariates.

Figure 11 provides a summary of the findings based on the results of research questions $5,6,7,8 \& 9$. The figure demonstrates all the significant associations that were obtained. Thus, specialty of internal medicine and more number of repeat AMI patients treated was associated with higher absolute contraindication score. The specialty of cardiology, younger age of physician and university affiliation was associated with higher not a contraindication score. Younger age, and university affiliation were also associated with higher definitely prescribe score, in addition to larger size of practicing hospital, and internal medicine specialty. Larger size of practicing hospital, internal medicine specialty and practicing in a hospital was associated with higher maybe prescribe score. Among all the tested variables, not a contraindication score, definitely prescribe score, maybe prescribe score, definitely not prescribe score, younger age and larger size of hospital were all associated in univariate analysis with higher prescribing rates or 
prescribing behavior. However, when all these variables were entered in a multivariate model, definitely prescribe score was the only significant variable.

The knowledge scores, willingness scores and prescribing behavior, were all proxy variables. Thus, the findings of this phase indicate a profile of a general specialty/family practice physician, older in age, non-university or non-hospital affiliated and attached to a smaller hospital as the target for interventions to improve beta-blocker prescribing behavior. 
Figure 11: Summary of Univariate and Multivariate Findings

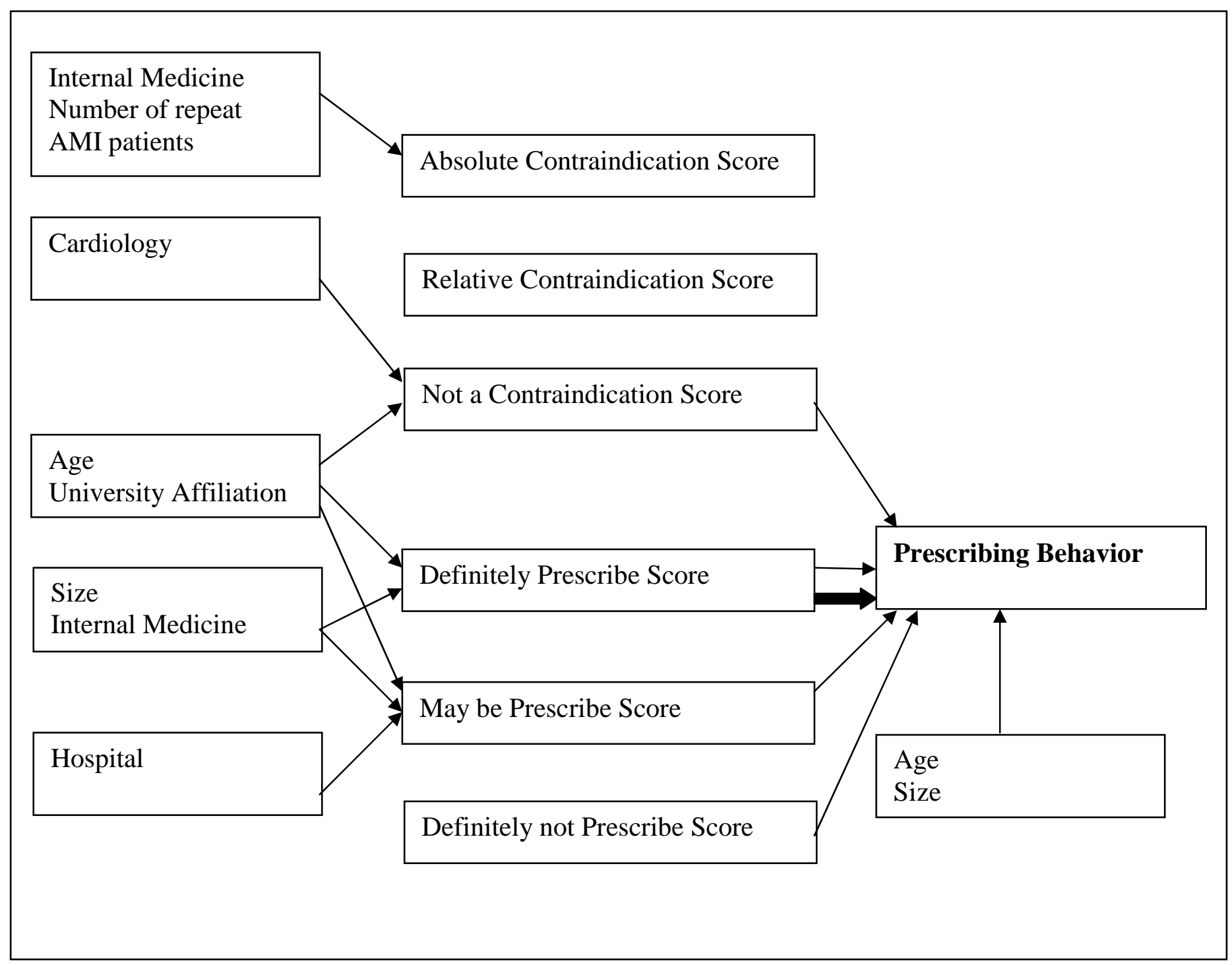

AMI: Acute myocardial infarction, Univariate association, Multivariate association 


\section{Results for Research Question 10}

This included a descriptive summarization of the various reasons reported by physicians for not prescribing beta-blockers in their post-AMI patients. It was an openended question and most physicians reported multiple reasons. These reasons were broadly categorized based on their similarities. For example bronchospasm, COPD, severe COPD, lung disease, and severe lung disease were included in one category and called as bronchospasm/COPD/lung disease. Similarly, contraindications, relative contraindication, partial contraindication, intolerance, cannot tolerate, complications were grouped together in contraindications/complications/intolerance.

The most important reasons reported by physicians for not prescribing betablockers as post-AMI medication were bronchospasm/COPD/lung disease, Bradycardia/bradyarrhythmia, and contraindications/complications/intolerance (Table 45). Among other reasons reported some included relative contraindications such as asthma/bronchial asthma, heart block, low blood pressure, low heart rate, pulse rate, peripheral vascular disease, $\mathrm{CHF}$ or low $\mathrm{EF}$, and diabetes. A few reported hypotension, an absolute contraindication. A few reported side effects or adverse effects as the general cause and one physician reported fatigue and another reported non-compliance as a cause. Very few physicians reported co-morbidity, cost, depression, and severe systolic dysfixia as a reason. The non-specific reasons were grouped in the other category and included-already on medication, don't treat MI, forgot, did not think about it, medication has new indications, not in active patients, symptomatic, and patient transferred for care. 
Table 45: Reasons for Not Prescribing Beta-blockers in Post-AMI Patients

\begin{tabular}{llll} 
Reason 1 (n= 116) & Reason 2 $(\mathbf{n = 5 7 )}$ & Reason 3 $(\mathbf{n}=\mathbf{1 3})$ & Reason $4(\mathbf{n}=\mathbf{3})$ \\
\hline $\begin{array}{l}\text { Bronchospasm/COP } \\
\text { D/lung disease (28) }\end{array}$ & $\begin{array}{l}\text { Bronchospasm/COP } \\
\text { D/lung disease (11) }\end{array}$ & Diabetes (2) & CHF/ low EF (1) \\
$\begin{array}{l}\text { Bradycardia/ } \\
\text { Bradyarrhythmia (20) }\end{array}$ & $\begin{array}{l}\text { Contraindications/ } \\
\text { complications/intole } \\
\text { rance (10) }\end{array}$ & Low BP (3) & Depression (1) \\
$\begin{array}{l}\text { Contraindications/co } \\
\text { mplications/intoleran } \\
\text { ce (17) }\end{array}$ & $\begin{array}{l}\text { Bradycardia/ } \\
\text { Bradyarrhythmia (8) }\end{array}$ & $\begin{array}{l}\text { Bronchospasm/CO } \\
\text { PD/lung disease (1) }\end{array}$ & $\begin{array}{l}\text { Side/adverse } \\
\text { effects (1) }\end{array}$ \\
$\begin{array}{l}\text { Asthma/bronchial } \\
\text { asthma (13) }\end{array}$ & Hypotension (6) & $\begin{array}{l}\text { Bradycardia/ } \\
\text { Bradyarrhythmia }\end{array}$ &
\end{tabular}

(1)

Side/adverse effects Asthma/bronchial CHF/ low EF (1)

(7) asthma (4)

Hypotension (5) Heart block (4) Cost (1)

Low heart rate (5) Low pulse (3) Heart block (1)

Low BP (4) Side/adverse effects Low heart rate (1) (3)

Heart block (3) Low BP (2) Peripheral Vascular disease (1)

Co-morbidity (2) Low heart rate (2) Side/adverse effects (1)

CHF/ low EF (1) Diabetes (1)

Fatigue (1) Peripheral Vascular disease (1)

Non compliance (1) Low EF (1)

Peripheral Vascular Others (1) disease (1) 
Table 45: Reasons for Not Prescribing Beta-blockers in Post-AMI Patients (contd.)

Reason $1(n=116) \quad$ Reason $2(n=57) \quad$ Reason $3(n=13) \quad$ Reason $4(n=3)$

Severe systolic

dysfixia (1)

Others (7)

Others: Already on medication, don't treat AMI, forgot, did not think, new indications, not in active patients, symptomatic, transferred for care

AMI: Acute myocardial infarction, n: Number of physicians, COPD: Chronic obstructive pulmonary disease, BP: Blood pressure, CHF: Congestive heart failure, EF: Ejection fraction 


\section{Discussion for Research Question 10}

The above summarization reveals that the most common reasons reported for not prescribing beta-blockers as post-AMI medications were COPD, bradycardia and contraindications. Bradycardia is an absolute contraindication of beta-blockers and is a correct reason for not prescribing as per the guidelines. However, COPD is a relative contraindication and patients should be prescribed beta-blocker therapy, thus this suggests inappropriate prescribing behavior. Also, the third most common reason given by physicians was contraindications, most physicians did not specify what the contraindication was. Thus, these could be relative contraindications or absolute contraindications, but due to lack of sufficient information it cannot be determined whether the reasons were appropriate or not. The other frequently mentioned reasons were asthma, hypotension, low heart rate, and adverse or side effects of therapy. Asthma and low heart rate are relative contraindications and not adequate reasons for not prescribing. Hypotension is an absolute contraindication and thus a correct reason for not prescribing as per the guidelines. Adverse reactions have been reported to be a barrier for prescribing beta-blockers in a previous study too (Kennedy \& Rosensen, 1995). This study reported that physicians believed that adverse reactions of beta-blockers could result in a negative quality of life. This study also reported exaggerated concerns for relative contraindications. Thus, based on the previous report by Kennedy and Rosensen and present findings, it appears as though physicians' reasons for not prescribing betablockers in post-AMI patients for secondary prevention haven't changed much. This is despite additional studies which have reported that beta-blockers can be used in all patients except those with absolute contraindications (Phillips et al., 2000). This gives 
rise to the need of increasing awareness of these newer study reports to physicians who attend to post-AMI patients and thus increase prescribing rates of beta-blockers in postAMI patients. A few physicians in this study also reported reasons such as forgot, and did not think. Effective reminder cards or services could be beneficial in increasing prescribing rates of such physicians. Thus, this summarization illustrates that reasons given by physicians for not prescribing beta-blockers may not necessarily be adequate and there is room for improving prescribing rates of this important therapy in post-AMI patients.

This chapter presented results and discussion for each research question that was proposed by the study. The next chapter will give summarization and make conclusion from these results. 


\section{CHAPTER FIVE}

\section{SUMMARY AND CONCLUSIONS}

This chapter presents a review of the study findings, draws conclusions, presents research implications, lists limitations for each phase of the study, and provides recommendations for future research.

\section{Phase I}

Acute myocardial infarction (AMI) is a condition responsible for high mortality and has a lot of economic implications. Hence, therapeutic interventions that reduce mortality and improve morbidity, as well as primary and secondary prevention strategies for an AMI are important. Beta-blocker therapy has been proven to be effective when used as a secondary preventive agent following an AMI. Due to its effectiveness, it has been recommended by American College of Cardiology (ACC)/ American Heart Association (AHA) guidelines for long-term use following an AMI. However, this important therapy has been reported to be inappropriately prescribed (underused and misused) in patients following an AMI. There have been a few investigations to understand the consequences of under use of this therapy. However, very little has been done to understand the effect of inappropriate use. Also, previous research has been in an elderly population, despite the fact that this condition is present in younger age groups.

Thus, the goal of this phase of the study was to evaluate the prescribing of betablockers as long-term therapy following an AMI in a Medicaid population. Another aim was to investigate the effect of appropriate and inappropriate prescribing of beta-blockers 
following an AMI on patient outcomes such as mortality, morbidity, health care utilization and expenditures.

\section{Conclusions for Phase I}

The conclusions for this phase of the study are presented based on the research questions proposed in the study:

\section{Research Question 1: Prescribing Patterns for Beta-blocker Therapy in WV Medicaid}

This was an exploratory question to assess prescribing of beta-blockers in the Medicaid population with regards to the published guidelines. The prescription rates were higher in eligible patients and lower in ineligible patients, compared to prescription rates reported in previous research. Another important observation was that there was a tendency to not prescribe in older patients. All these findings suggest that prescribing in this patient population is better that the previously reported prescribing in other populations, but there is abundant room for improvement in prescribing.

Research Question 2: Effect of Appropriate and Inappropriate Prescribing on Mortality

The null hypothesis this research question aimed to investigate was that there is no difference in mortality and cardiac mortality between the appropriately and inappropriately prescribed groups after controlling for confounding factors. Appropriate therapy with beta-blockers did affect all-cause mortality. The appropriate group had lower mortality compared to the inappropriate group. However, appropriate therapy was 
not significant when cardiac mortality was investigated. Thus, appropriate therapy did not affect cardiac mortality in this cohort of patients.

Research Question 3: Effect of Appropriate and Inappropriate Prescribing on Morbidity

The null hypothesis this research question aimed to investigate was that, there is no difference in morbidity due to: successive AMI, heart failure, stroke, angina, other ischemic disease, essential hypertension, cardiogenic shock, disease of endocardium, conduction disorders or cardiomyopathy between the appropriately and inappropriately prescribed groups after controlling for confounding factors. Appropriate beta-blocker therapy did affect morbidity due to these conditions. The inappropriate group had better survival compared to the appropriate group in the 12-month follow-up period in this patient population. The patients in the appropriate group were twice as likely to suffer from a cardiac event in the follow-up period compared to the inappropriate group after adjusting for all the co-variates available in the claims. However, there were indications that the appropriate group had a more severe condition and thus in a worse health state during the hospitalized AMI compared to the inappropriate group. Thus, whether the increase in morbidity in the follow-up period was because of appropriate therapy or because of patient severity could not be determined.

Research Question 4: Effect of Appropriate and Inappropriate Prescribing on Utilization and Expenditure

The null hypothesis this research question aimed to investigate was that, there is no difference in utilization of resources and expenditure due to successive AMI, heart 
failure, stroke, angina, other ischemic disease, essential hypertension, cardiogenic shock, disease of endocardium, conduction disorders or cardiomyopathy between the appropriately and inappropriately prescribed group after controlling for confounding factors. Appropriate beta-blocker therapy did affect utilization, but it did not affect expenditures. The inappropriate group had lower utilization-hospital visits, ER visits, and total length of stay in the 12-month follow-up period in this patient population. However, there were indications that the appropriate group had a more severe condition and thus in a worse health state during the incident AMI compared to the inappropriate group. Thus, whether the increase in utilization was because of appropriate therapy or because of patient severity could not be determined.

\section{Phase II}

This phase of the study involved exploring the association of physician characteristics such as physician knowledge of contraindications, physicians' willingness to prescribe and demographic and practice characteristics with their prescribing of betablocker therapy as a post-AMI secondary preventive agent. Previous studies have reported ample variation of medication use by different physician characteristics. This phase of the study aimed to understand effect of these factors individually and in combination with prescribing behavior in the state of WV for beta-blockers in post-AMI patients. 


\section{Conclusions for Phase II}

The conclusions of this phase of the study are presented based on the research questions proposed in the study:

Research Question 5: Knowledge of Contraindications and Prescribing Behavior

The null hypothesis this research question aimed to investigate was that, there is no relationship between knowledge of contraindications and prescribing behavior for beta-blocker therapy in post AMI patients. Physicians' knowledge of both absolute and relative contraindications did not reveal any association with prescribing behavior. On the other hand, knowledge of conditions which were not contraindications was positively associated with prescribing behavior.

\section{Research Question 6: Willingness to Prescribe and Prescribing Behavior}

The null hypothesis this research question aimed to investigate was that, there is no relationship between willingness to prescribe beta-blockers and prescribing behavior

for beta-blocker therapy in post AMI patients. Physicians' willingness to prescribe betablockers was positively associated with their prescribing of this therapy in post-AMI patients as secondary preventive agents irrespective of patient co-morbidity.

\section{Research Question 7: Demographic and Practice Characteristics and Prescribing Behavior}

The null hypothesis this research question aimed to investigate was that, there is no relationship between demographic and practice characteristics and prescribing behavior for beta-blocker therapy in post AMI patients. Of the various characteristics 
investigated age and size of hospital were associated with physician prescribing of betablocker in post-AMI patients as secondary preventive agents. Age was negatively associated with prescribing behavior and size of the hospital was positively associated with prescribing behavior.

\section{Research Question 8: Knowledge of Contraindications, Willingness to Prescribe and Demographic and Practice Characteristics}

The null hypothesis this research question aimed to investigate was that, there is no relationship between demographic and practice characteristics and knowledge of contraindications and willingness to prescribe beta-blocker therapy in post AMI patients. Of the various characteristics investigated specialty, practice site, age, and number of repeat AMI patients treated by physicians were associated with physicians' knowledge of contraindications. Similarly, specialty, practice site, age, and size of hospital were associated with willingness to prescribe beta-blockers as secondary preventive agent in post-AMI patients. Age was negatively associated with both knowledge and willingness to prescribe scores. Primary practitioners from family practice and general practice generally had lower knowledge and willingness to prescribe scores. University affiliation of the practice site was associated with higher knowledge and willingness to prescribe scores. More number of repeat AMI patients treated was associated with lower knowledge score and larger size of the practice hospital was associated with higher willingness to prescribe score. 


\section{Research Question 9: Physician Characteristics and Prescribing Behavior}

The null hypothesis this research question aimed to investigate was that, there is no relationship of prescribing behavior and physician characteristics such as demographics, practice characteristics, knowledge of contraindications, and willingness to prescribe beta-blocker therapy in post AMI patients. The study revealed that when all physician characteristics are controlled for, physician willingness to prescribe is the most significant predictor of physicians' prescribing behavior. However, when the various associations were individually assembled; age, specialty, university affiliation and size of the hospital emerged to be the modifiable factors which can be targeted with educational efforts to increase prescribing of beta-blockers in post-AMI patients.

\section{Research Question 10: Reasons for Not Prescribing Beta-Blockers}

This was an exploratory question to enlist reasons reported by physicians for not prescribing beta-blockers in post-AMI patients. The most common reasons were COPD, bradycardia, and contraindications. Comparison of the reasons with those reported in previous reports revealed that though prescribing has increased over the years, the reasons for not prescribing haven't changed much, thus there is a need to increase awareness which would further improve prescribing of beta-blockers as secondary preventive agents in post-AMI patients.

\section{Implications of Study}

This study was undertaken to study prescribing of beta-blockers as post-AMI secondary prevention agents and its effects on patient outcomes in a Medicaid 
population. The study also aimed to assess the physicians' role in prescribing betablockers following an AMI. The findings of the study should be useful to the Medicaid program to help them improve prescribing of beta-blockers for post-AMI long-term use. The study also has implications for patients, prescribers and decision-makers. These implications are described in the following sections.

\section{Implication to the Medicaid Program}

The results of this study demonstrated that there is indeed inappropriate prescribing of beta-blockers in this patient cohort of Medicaid. There is both under use and misuse. Inappropriate use of an important therapeutic agent can have adverse consequences on patient outcomes. This study revealed that appropriate use of betablocker therapy is associated with a significant lower mortality rate. Thus, this further stresses the importance of increasing appropriate prescribing of beta-blockers in these patients. However, the results did not show a significant association of appropriate prescribing with cardiac mortality and also revealed that patients with appropriate prescribing were associated with higher morbidity and utilization of resources. But it was not clear whether this increase in morbidity and utilization was associated with appropriate use of beta-blockers or patient severity. And the data suggests that patient severity maybe responsible for higher utilization. Thus, there is a need to improve appropriate prescribing of beta-blockers in these patients. The Medicaid program can develop and implement active interventions such as group or one-on-one education, and individual outreach visits that will increase the prescription of beta-blockers in eligible patients, and decrease the prescription of beta-blockers in ineligible patients. This could 
improve patient quality of life and reduce healthcare utilization and costs, as this would result in better quality of care, fewer deaths, better control of symptoms and exacerbations due to cardiovascular (CV) disease.

\section{Implication to Patients}

The findings of this study are also relevant to patients. AMI is a condition that affects quality of life, productivity, incurs high expenses to patients (Example; insurance plans where patients pay a 80:20 deductible) and can result in death. This study is one of the primary attempts to investigate outcomes of AMI in a younger population. Although no significant difference was demonstrated, mortality was lower in the appropriate group for cardiac conditions. Hence, increasing awareness of importance of beta-blockers among patients would result in patients asking their physicians about prescribing this therapy (they may want to know about contraindications). This will eventually lead to increases in appropriate prescribing and thus better outcomes (postponement of death) for AMI patients.

\section{Implications to Prescribers}

The study demonstrated lower mortality in the appropriately prescribed group. This will help those physicians who are skeptical about prescribing beta-blockers in patients with low risk, or presence of relative contraindications increase prescribing, and those physicians who prescribe in patients with absolute contraindications decrease prescribing. Both these scenarios will lead to increase in appropriate prescribing behavior. 


\section{Implications to Decision-Makers}

Decision-makers can benefit from this study in two ways. First, the study shows that beta-blockers are being inappropriately prescribed. Thus, they can develop and implement strategies which will increase appropriate prescribing of beta-blockers. Secondly, the study shows that a physician willingness to prescribe affects their prescribing behavior, and is dependent on their demographics, practice characteristics, and their knowledge about guidelines. Thus, they can target physicians who are older, from general practice, who work in solo settings or who work in small hospitals with interventions which will increase their willingness to prescribe beta-blockers. Ultimately, this should affect their prescribing of beta-blockers in post AMI patients.

\section{Limitations of Study}

The study had two phases, phase I used secondary data and phase II used primary data collection. Both phases have limitations, these are discussed in the following sections:

\section{Limitations of Phase I}

The study group for the phase was continuously eligible recipients of Medicaid, and excluded managed care and/or Medicare recipients. Beta-blockers are known to provide more benefits for high-risk patients. Age greater 65 is one of the high-risk criteria (ACC/AHA, 1996). Thus, a significant portion of those individuals, who would probably have more benefits of appropriate prescribing, were eliminated in the study due to unavailability of all utilization data. Also, since managed care recipients and those 
recipients who were not continuously eligible were excluded, the results cannot be generalized for these excluded patients.

The study used data collected for claims purposes and not research purposes, thus, errors due to billing and coding cannot be ruled out. Severity indicators which would have helped to adjust for patient risk were not available. Also, some of the relative contraindications could not be identified such as peripheral hypoperfusion, and intolerance or allergy to beta-blockers.

The death records obtained from Vital Statistics did not contain detailed information about the patients. This information would have helped determine the true cause of death and aid in deciding whether the deaths were cardiac or non-cardiac. Due to limitations of this missing information, a lot of deaths were excluded from the cardiac death investigation and the result for cardiac mortality was not significant.

Patients are generally advised to modify certain life-style related characteristics that affect their risk for future cardiac morbidity. These include smoking cessation, cardiac rehabilitation, exercise, and dietary modifications. This information is not available in Medicaid claims and could not be adjusted for in the analysis used.

\section{Limitations of Phase II}

This phase involved 1,019 physicians associated with post-AMI care. However, only a response rate of 25.61 percent was obtained. The non-response bias analysis revealed that the respondents and the non-respondents were significantly different from each other on some of the variables compared. There are chances that they can also be different in their prescribing behavior, knowledge and willingness to prescribe and thus 
the effect of non-response bias cannot be completely eliminated. This limits generalization of the results to the entire state. Additionally, the survey was conducted in the state of WV. The physicians in this state may have different characteristics compared to physicians in other states. Thus, the results may not be as easily extrapolated to other regions.

Another limitation of this phase is the way the instrument was designed. The respondents had to give their willingness to prescribe beta-blocker therapy for patients with different conditions. However, in clinical settings, patients may not have just one of these conditions. They can have multiple conditions, with different severity levels. These conditions will influence the prescribing of the physicians. This phase also suffers from another drawback-the absence of patient preferences. With side effects of betablockers such as decreased libido, impotence, and fatigue there is a possibility that patients' preference will affect physician prescribing. Also, beta-blocker prescribing percent or prescribing behavior was estimated based on prescription of beta-blockers to the last 10 post-AMI patients treated by the physicians. Treatment of the last 10 patients may not be representative of the physicians' general prescribing behavior for betablockers in post-AMI patients. Also, it could not be determined through the survey whether the prescribing of the physicians for the last 10 patients was appropriate or inappropriate. However, due to time and resource constraints this method of getting a proxy for prescribing behavior appeared to be the best. Another discrepancy was the fact that the guidelines classify atrioventricular (AV) block as both relative and absolute contraindication, hence there is uncertainty whether to classify it as an absolute contraindication or relative contraindication. For study purposes, first degree AV block 
was considered to be a relative contraindication and second and third degree AV blocks were considered to be absolute contraindications.

Based on the survey's estimation, greater than $90 \%$ of the physicians were prescribing beta-blockers to their post-AMI patients. Phase I of the study which investigated beta-blocker prescribing for WV Medicaid demonstrated a 64\% prescribing rate. Recent data reported by Schade et al., (2002) for the WV Medicare population, reported 59.4\% prescribing rates for beta-blockers in Medicare AMI patients. Both these rates are well below the rates obtained from the survey. Thus, it is likely that the respondents of the survey gave desirable responses and this introduces bias due to selfreported data collection method.

Finally, other limitations of using a self-administered mail questionnaire for data collection would apply for this study. Although mail questionnaires possess the advantages of being relatively inexpensive to administer, access to larger population, anonymity, and elimination of interviewer bias, there are substantial limitations. These include measurement errors due to respondents not understanding the instructions and items, item non-response, insensitivity to substitution, and recall bias.

\section{Directions for Future Research}

The two phases in this study have given rise to several interesting questions. These questions will provide the impetus for future research purposes. The simplest study that arises from phase I involves a prospective study evaluating utilization of betablockers and its effects on patient outcomes. The inability of this phase to adjust for patient severity and risk levels and the inability to adjust for life-style modifications can be adjusted by undertaking a prospective study where patients with AMI will be 
identified from one or multiple health systems and followed for a pre-specified duration of time to determine outcomes such as mortality, morbidity, utilization and expenditures. Patient chart information can be used to adjust for severity and patients can be interviewed to know any life-style modification/s undertaken.

Another limitation of the study was the inability to determine beta-blocker use and its effects on outcomes in the elderly. Linking Medicaid and Medicare records will allow inclusion of this cohort and thus extending the results of phase I of this study and hence should be pursued in future.

By conducting the study in Medicaid, phase I of the study involved younger patients who belong to lower socio-economic status. It would be interesting to duplicate this study in a third party setting, where the patients would be in a comparable age group to Medicaid and belong to a higher socio-economic class.

Phase II of the study provided current knowledge and willingness to prescribe among physicians. Interventions should be designed to educate the physicians about the gaps in their knowledge and a post study should be undertaken to find out the effectiveness of such interventions. This would help design interventions that enhance adherence to practice guidelines.

And finally, since there hasn't been much reported from the patient's perspective, a study that measures and compares effect of beta-blocker therapy on patients' quality of life in patients with AMI should be undertaken. 


\section{BIBLIOGRAPHY}

American College of Cardiology (ACC)/American Heart Association (AHA) (1996). Guidelines for the management of patients with acute myocardial infarction. Journal of American College of Cardiology, 28(5), 1328-428.

American College of Cardiology (ACC)/American Heart Association (AHA) (1999). Guidelines for the management of patients with acute myocardial infarction. A report of the American College of Cardiology/American Heart Association Task Force on Practice. Journal of American College of Cardiology, 34(3), 890-911.(\&/or) New recommendations from the 1999 American College of Cardiology/American Heart Association acute myocardial infarction guidelines. Annals of Pharmacotherapy, 35(5), 589-617.

Anis, A. H., Lynd, L. D., Wang, X. King, G., Spinelli, J. J., Fitzgerald, M., Bai, T., \& Pare, P. (2001). Double trouble: Impact of inappropriate use of asthma medication on the use of health care resources. Canadian Medical Association Journal, 164(5), 625631.

Agusti, A., Arnau, J.M., \& Laporte, J.R. (1994). Clinical trials versus clinical practice in the secondary prevention of myocardial infarction. European Journal of Clinical Pharmacology, 46(2), 95-9.

Ahdout, D.J., Daman, P.M., \& Ultan, L.B. (1989). Recurrent acute pulmonary emboli in association with acute myocardial infarction. Chest, 96(3), 682-4.

Ayanian, J.Z., Hauptman, P.J., Guadagnoli, E., Antman, E.M., Pashas, C.L., \& McNeil, B.J. (1994). Knowledge and practices of generalists and specialist physicians regarding drug therapy for acute myocardial infarction. New England Journal of Medicine, 331(17), 1136-42.

Bellenir K (2000). Heart diseases and disorders Source book. 2nd edition. Omnigraphics, Inc.

Benfield, P., Clissold, S.P., \& Brogden, R.N. (1986). Metoprolol: An update review of its pharmacodynamic and pharmacokinetic properties, and therapeutic efficacy, in hypertension, ischaemic heart disease and related cardiovascular disorders. Drugs, 31(5), 376-429.

Bero, L.A., Lipton, H.L., \& Bird, J.A. (1991). Characterizations of geriatric drugrelated readmissions. Medical Care, 29(10), 989-1003.

Bradford, W.D., Chen, J., \& Krumholz, H.M. (1999). Under-utilization of betablockers after acute myocardial infarction, pharmacoeconomic implications. Pharmacoeconomics, 15(3), 257-268. 
Brand, D.A., Newcomer, L.N., Freiburger, A., , \& Tian, H. (1995). Cardiologists' practices compared with practice guidelines: Use of beta-blockade after acute myocardial infarction. Journal of American College of Cardiology, 26(6), 1432-6.

Camm, A., J., Ward, D.E., \& Whitmarsh, V. B. (1982). The acute electrophysiological effects of intravenous metoprolol. Clinical Cardiology, 5(15), 32731.

Carson, J.L., Kelly, M.A., Duff, A., Fulkerson, W.J., Palevsky, J.S, Schwartz, J.S. et el. (1992) The clinical course of pulmonary embolism. New England Journal of Medicine, 326(19), 1240-1245.

Chadda, K., Goldsetin, S., Byinton, R., \& Curb, J.D. (1986) Effect of propranolol after acute myocardial infarction in patients with congestive heart failure. Circulation, 73(3), 503-10.

Chen, J., Radford, J., Wang, Y., Marciniak, T.R.A. \& Krumholz, H.M .(2000). Are beta-blockers effective in elderly patients who undergo coronary revascularization after acute myocardial infarction. Archives of Internal Medicine, 160(7), 947-952.

Chao, C.S. (2002). Physicians attitudes toward DNR of terminally ill cancer patients in Taiwan. Journal of Nursing Research, 10(3), 161-7.

Communiqué (2001). Underwriting young patients after myocardial infarct in the thrombolytic era. Available at: www.underwriting.co.za/communique/issue\%203/newpage3.

Coughlin, T.A., Ku, L., \& Holahan, J. (1994). Medicaid since 1980: costs, coverage, and the shifting alliance between the federal government and the states. Washington, D.C. The Urban Institute Press.

Cruickshank, J.M., \& Princhard, B.N.C. (1994). Beta blockers in clinical practice. $2^{\text {nd }}$ ed. New York (NY): Churchill Livingstone, 559-68.

D'Agnostino, R.B. (1998). Propensity score methods for bias reduction in the comparison of a treatment to a non-randomized control group. Statistics in Medicine, 17, 2265-2281.

Dillman D. (1978). Mail and Telephone Survey: The Total Design Method. New York: John Wiley \& Sons.

Doughty, M., Mehta, R., Bruckman, D., Das, S., Karavite, D., Tsai, T., \& Eagle, K. (2002). Acute myocardial infarction in the young-The university of Michigan experience. American Heart Journal, 143(1), 56-62. 
Edvardsson, N., \& Olsson, S.B. (1981). Effects of acute and chronic beta receptor blockade on ventricular repolarization in man. British Heart Journal, 45(6), 628-36.

Fehrenbach, S.N., Budnitz, D.S., Gazmararian, J.A., \& Krumholz, H.M. (2001). Physician characteristics and the initiation of beta-adrenergic blocking agent therapy after acute myocardial infarction in a managed care population. American Journal of Managed Care, 7(7), 717-723.

Figueiras, A., Sastre, I., \& Gestal-Oterao, J. (2001). Effectiveness of education interventions on the improvement of drug prescription in primary care: a critical literature review. Journal of Evaluation in Clinical Practice, 7(2), 223-241.

Fishkind, D., Paris, B.E., \& Aronow, W.S. (1997). Use of digoxin, diuretics, beta-blockers, angiotensin-converting enzyme inhibitors and calcium channel blockers in older patients in an academic hospital-based geriatrics practice. Journal of American Geriatric Society, 45(7), 809-12.

Frishman, W.H., \& Cheng A. (1999). Secondary prevention of myocardial infarction: Role of beta adrenergic blockers and angiotensin converting enzyme inhibitors. American Heart Journal, 137(4pt3), S25-34.

Frishman, W., \& Silverman, R. (1979). Appraisal and reappraisal of cardiac therapy. Clinical pharmacology of the new beta-adrenergic blocking drugs. Part 2. Physiologic and metabolic effects. American Heart Journal, 97(6), 797-807.

Furberg, C.D. \& Byington, R.P. (1983). What do subgroup analyses reveal about differential response to beta-blocker therapy? Circulation, 67(6pt2), I98-101.

Gold, M.R., Dec, G.W., Cocca-Spofford, D., \& Thompson, B.T. (1991) Esmolol and ventilatory function in cardiac patients with COPD. Chest, 100(5), 1215-8.

Goldman, L., Sia, B.S.T., Cook, E. F., Rutherford, J.D., \& Weinstein, M.C. (1988). Costs and effectiveness of routine therapy with long-term beta-adrenergic antagonists after acute myocardial infarction. New England Journal of Medicine, 319(3), $152-7$.

Goldstein, S. (1996). Beta-blockers in hypertensive and coronary heart disease. Archives of Internal Medicine, 156(12), 1267-76.

Gottlieb, S.S. \& McCarter, R.J. (2001). Comparative effects of three betablockers (atenolol, metoprolol, and propranolol) on survival after acute myocardial infarction. American Journal of Cardiology, 87(7), 823-26.

Gotleib, S.S., McCarter, R.J., \& Vogel, R.A. (1998). Effect of beta-blockade on mortality among high-risk and low-risk patients after myocardial infarction. New England Journal of Medicine, 339(8), 489-497. 
Gurwitz, J.H., Goldberg, R.J., Chen, Z., Gore, J.M., \& Alpert, J.S. (1992). Beta blocker therapy in acute myocardial infarction: evidence for under-utilization in the elderly. American Journal of Medicine, 93(6), 605-19.

Halverson, R., Palmquist, R. (1980). The interpretation of dummy variables in semi-logarithmic equations. American Economic Reviews, 70, 474-5.

Heller, D.A., Ahern, F.M., \& Kozak, M. (2000). Changes in rates of bet-blocker use between 1994 and 1997 among elderly survivors of acute myocardial infarction. American Heart Journal, 140(9), 663-71.

Herlitz, J., Dellborg, M., Karlson, B.W., Lindqvist, J. \& Wede, 1.H. (2000). Longterm mortality after myocardial infarction in relation to prescribed dosages of a betablocker at hospital discharge. Cardiovascular Drugs and Therapy, 14(6), 589-95.

Health Care Financing Administration (HCFA), 1997. Center for Medicaid State Operations. HCFA-2082 Report.

Health Care Financing Administration (HCFA), 2000. Medicaid Statistical Information System (MSIS). Source: HCFA CMSO, HCFA 2062 Report. Available at: www.hcfa.gov/medicaid/msis/mcd98t02.pdf.

Hjalmarson, A. (1988). International beta-blocker review in acute and post myocardial infarction. American Journal of Cardiology, 61(3), 26B-9B.

Huse, D. M., Roht, L. H., Alpert, J. S., \& Hartz, S. C.(2001). Physicians' knowledge, attitudes, and practice of pharmacologic treatment of hypertension. Annals of Pharmacotherapy, 35(10), 1173-9.

Ishikawa, K., Miyataka, M., Kanamasa, K., Hayashi ,T., Takenaka, T., Inoki, T., Katayama, K., Kimura, A., Yabushita, H., \& Kitayama, K. (2000). Retrospective analysis showing less cardiac events in post myocardial infarction patients treated with metoprolol. Japanese Circulation Journal, 64(5), 358-64.

Jollis, J.G., Delong, E.R., Peterson, D., Muhlbaier, L.H., Fortin, D.F., Califf, R.M., \& Mark, D.B. (1996). Outcomes of acute myocardial infarction according to the specialty of the admitting physician. New England Journal of Medicine, 335(25), 1880-7.

Kennedy, P. (1981). Estimation with correctly interpreted dummy variables in semi-logarithmic equations (letter). American Economic Review, 71, 801.

Kennedy, H.L., \& Rosenson, R.S.(1995). Physician use of beta-adrenergic blocking therapy: a changing perspective. Journal of American College of Cardiology, 26(2), 547-52. 
Khan (2000). Beta-blockers: Cornerstone of Cardiac therapy. In Cardiac Drug therapy. Harcourt Publishers Limited. London.

Knapp, D.E., Knapp, D.A., Speedie, M.K., Yaeger, D.M. \& Baker, C.L. (1979). Relationship of inappropriate drug prescribing to increased length of hospital stay. American Journal of Hospital Pharmacy, 36(10), 1334-37.

Krumholz, H.M., Radford, M.J., Wang, Y., Chen, J., Heiat, A., \& Marciniak, T.A. (1998). National use and effectiveness of beta-blockers for the treatment of elderly patients after acute myocardial infarction. Journal of American Medical Association, 280(7), 623-629.

Linden, T., Carmejo, G., Wiklund, O., Warnold, I., Olofsson, S.O., \& Bondjers, G. (1988). Effect of short-term beta-blocker on serum lipids and on the interaction of LDL with human arterial proteoglycan: A comparison between metoprolol-LR and atenolol. In Linden T. Doctoral thesis. Retention and accumulation of apolipoprotein B and lipids in human arterial tissue. Vassastagends, Goteborg.

Lindley, C.M., Tully, M.P., Paramsothy, V., \& Tallis, R.C. (1992). Inappropriate medication use is a major cause of adverse drug reactions in elderly patients. Age and Aging, 21(4), 294-300.

McDervitt, D.G. (1983). Clinical significance of cardioselectivity. State of the art. Section 5. Cardioselectivity. Drugs, 25, 219-26.

McCormik, D., Gurwitz, J.H., Lessard, D., Yarzebski, J., Gore, J.M., \& Goldberg, R.J. (1999). Use of aspirin, Beta-blockers and lipid-lowering medications before recurrent acute myocardial infarction. Archives of Internal Medicine, 159(6), 561-67.

Medicaid Consumer Information (2003). Available:http://cms.gov/medicaid/consumer.asp. Accessed 2/25/2003.

Meehan, T.P., Hennen, J., Radford, M.J., Petrillo, M.K., Elstein, P., \& Ballard, D.J. (1995). Process and outcome of care for acute myocardial infarction among Medicare beneficiaries in Connecticut: A quality improvement demonstration project. Annals of Internal Medicine, 122(12), 928-936.

Mehta, S. S., Wilcox, C. S., \& Schulman, K. A. (1999). Treatment of hypertension in patients with comorbidities: Results from the study of hypertensive prescribing practices. American Journal of Hypertension, 12(4 pt 1), 333-40.

Momani, A. (1999). Assessment of the impact of the West Virginia Medicaid's prior authorization policy for NSAIDs on chronic patients: economic and humanistic outcomes. Unpublished Dissertation, West Virginia University. 
Monette, J., Tamblyn, R.M., McLeod, P. J., \& Gayton, D. C. (1997). Characteristics of physicians who frequently prescribe long-acting benzodiazepines for the elderly. Evaluation and the Health Professions, 20(2), 115-30.

Mooss, A.N., Hilleman, D.E., Mohiuddin, S.M., \& Hunter, C.B. (1994). Safety of Esmolol in patients with acute myocardial infarction treated with thrombolytic therapy who had relative contraindications to beta-blocker therapy. Annals of Pharmacotherapy, 28(6), 701-3.

Mitra, S., Findley, K., Frohnapple, D. \& Mehta, J.L. (2002). Trends in long term management of survivors of acute myocardial infarction by cardiologist in a government university-affiliated teaching hospital. Clinical Cardiology, 25(1), 16-18.

Murray, D.P., Murray, R.G. \& Littler, W.A. (1986). The effects of metoprolol given early in acute myocardial infarction on ventricular arrhythmias. European Heart Journal, 7(3), 217-22.

Mussio (1987). Content Validity: A procedural Manual. Chicago: International Personnel Management Association.

National Committee for Quality Assurance. (1999). State of Managed Care Quality Report Washington, DC.

National Committee for Quality Assurance. (2000). State of Managed Care Quality Report. Available on: www.ncqa.org/somc2001/somc_2001_toc.html.

National Institute of Health (1995). Diabetes in America. NIH Publication No 95-1468. U.S government Printing office.

National Pharmaceutical Council (NCPA, 1995). Pharmaceutical benefits under state medical assistance programs. National Pharmaceutical Council, Inc. Reston VA.

National Pharmaceutical Council (NPCA, 1998). Pharmaceutical benefits under state medical assistance programs. National Pharmaceutical Council, Reston, VA.

National Registry of Myocardial Infarction- Quarterly Data Report, 1996.

Nicholls, S.J., McElduff, P., Dobson, A.J., Jamrozik, K.D., Hobbs, S.T. \& Leitch, J.W. (2001). Underuse of beta-blockers following myocardial infarction: a tale of two cities. Internal Medicine Journal, 31(7), 391-396.

Olsson, G., Ablad, B., \& Ryden, L. (1990). Long term cardiovascular effects of metoprolol therapy: a review article. Journal of Clinical Pharmacology, 30(2 suppl), S118-23. 
Olsson, G., Wikstrand, J., Warnold, I., Manger, C.V., McCoyle, D., Herlitz, J., Hjalmarson, A., \& Sonneblick, E.H. (1992). Metoprolol-induced reduction in postinfarction mortality: pooled results from five double blind randomized trials. European Heart Journal, 13(1), 28-32.

Olsson, G., \& Rehnqvist, N. (1984). Ventricular arrhythmia during the first year after myocardial infarction: influence of long-term treatment with metropolol. Circulation, 69(6), 1129-34.

Pashos, C.L., Normand, S.T., Garfinkle, J.B., Newhouse, J.P., Epstein, A.M., \& McNeil, B.J. (1994). Trends in the use of drug therapies in patients with acute myocardial infarction: 1988 to 1992. Journal of American College of Cardiology, 23(5), 1023-30.

Phillips, K.A., Shlipak, M.G., Coxson, P., Heindenreich, P.A., Hunink, M.G., Goldman, P.A, et al. (2000). Health and economic benefits of increased beta-blocker use following myocardial infarction. Journal of American Medical Association, 284(21), 2748-54.

Pilote, L., Califf, R.M., Sapp, S., Miller, D.P., Mark, D. B., Weaver, D., et al. (1995). Regional variation across the United States in the management of acute myocardial infarction. New England Journal of Medicine, 333(9), 565-72.

Pilote, L., Lavoie, F., Ho, V., \& Eisenberg, M.J. (2000). Changes in the treatment and outcomes of acute myocardial infarction in Quebec, 1988-1995. Canadian Medical Association Journal, 163(1), 31-36.

Plosker, G.L., \& Clissold, S.P. (1992). Controlled release metoprolol formulations: a review of their pharmacodynamic and pharmacokinteic properties, and therapeutic use in hypertension and ischaemic heart disease. Drugs, 43(3), 382-412.

Poldermans, D., Boersma, E., Bax, J.J., Thomson, I.R., Paelinck, B., van de Ven, L.L., et al (2001). Bisoprolol reduces cardiac death and myocardial infarction in highrisk patients as long as 2 years after successful major vascular surgery. European Heart Journal, 22(12), 1353-1358.

Podell, R.N. (1992). National guidelines for the management of asthma in adults. American Family Physician, 46(4), 1189-96.

Rathore, S.S., Beger, A.K., Weinfurt, K.P., Feinleib, M., Oetgen,W.J., Gersh, B.J. \& Schulman, K.A. (2000). Race, sex, poverty and the medical treatment of acute myocardial infarction in the elderly. Circulation, 102(6), 642-8.

Remohi, T. M., Arcos, S. A., Santos, R. B., Bautista, P. J., \& Aznar, G. (2000). Costs related to inappropriate use of albumin in Spain. Annals of Pharmacotherapy, 34(10), 1198-205. 
Rogers, W.J., Bowlby, L.J., Chandra, C.C, French, W.J., Gore, J.M, Lambrew, C.T, Rubinson, M. et al. (1994). Treatment of myocardial infarction in the United States (1990 to 1993). Circulation, 90(4), 2103-14.

Rogers, W.J., Canto, J.G., Lambrew, C.T., Tiefenbrunn, A.J., Kinkaid, B., Shoultz, D.A., et al (2000). Temporal trends in the treatment of over 1.5 million patients with myocardial infarction in the US from 1990 through 1999. Journal of the American College of Cardiology, 36(7), 2056-63.

Sanchez, G. F., Jernigan, D. B., Strausbaugh, L. J., Slemp, C. C., Perilla, M. J., \& Dowell, S. F. (2001). Treating cardiovascular disease with antimicrobial agents: A survey of knowledge, attitudes, and practices among physicians in the United States. Clinical Infectious Diseases, 33(2), 171-6.

Schade, C.P., Brehm, J.G., Stephens, M.K., \& Rezek, G. (2002). Beta-blocker use in acute myocardial infarction in West Virginia. West Virginia Medical Journal, 98(2), 56-60.

Schmander, K. E., Hanlon, J. T., Landsman, P. B., Samsa, G. P., Lewis, I. K., \& Weinberger, M. (1997). Inappropriate prescribing and health outcomes in elderly veteran outpatients. Geriatrics, 31(5), 529-33.

Shiekh, K., \& Bullock, C. (2001). Urban-rural differences in the quality of care for Medicare patients with acute myocardial infarction. Archives of internal medicine, 161(5), 737-743.

Shireman, T.I., Heaton, P. C., Gay, W. E., Cluxton, R. J., \& Moomaw, C.J. (2002). Relationship between asthma drug therapy patterns and health care utilization. Annals of Pharmacotherapy, 36(4), 557-64.

Sial, S.H., Malone, M., Freeman, J.L., Battiola, R., Nachodsky, J., \& Goodwin, J.S. (1994). Beta-blocker use in the treatment of community hospital patients discharged after myocardial infarction. Journal of General Internal Medicine, 9(11), 599-605.

Snow, P.J.D. (1965). Effect of propranolol in myocardial infarction. Lancet, 2(7412), 551-3.

Soumerai, S.B., McLaughlin, T.J., Spielgelman, D. Hertzmark, E., Thibault, G. \& Goldman, L. (1997). Adverse outcomes of under use of beta-blockers in elderly survivors of acute myocardial infarction. Journal of American Medical Association, $277(2), 115-21$.

Soons, K. R., Lynch, T., Seagrave, M., \& Roley, L. (1997). Determining physicians' knowledge and attitudes when prescribing drugs to treat gastrointestinal disorders. Clinical Performance and Quality Health Care, 5(2), 94-8. 
Soriano, J.B., Hoes, A.W., Meems, L., \& Grobbee, D.E. (1997) Increased survival with beta-blocks: importance of ancillary properties. Progress in Cardiovascular Diseases, 39(5), 445-56.

State of Managed Care Quality Report. Washington, DC: National Committee for Quality Assurance; 1999.

Stringer, K.A. \& Lopez, L.M. (1999). Myocardial Infarction. In Pharmacotherapy: A Pathological Approach. DiPiro JT, Talbert RL, Yee GC, Matzke GR, Wells BG, Posey LM (Eds). Appleton \& Lange.

Stroupe, K. T., Murray, M. D., Srump, T. E., \& Callahan, C. M., (2000). Association between medication supplies and healthcare costs in older adults from an urban healthcare system. Journal of American Geriatric Society, 48(7), 760-8.

Tsang, J., Sheppard, R., Mak, K.H., Brown. D., Huynh, T., Schechter. D., \& Eisenberg, M.J. (2002). Six-month outcomes of percutaneous transluminal coronary angioplasty in hypertensive patients: results for the ROSETTA registry. Routine Verses Selective Exercise Treadmill Testing After Angioplasty. American Heart Journal, 143(1), 124-9.

Vantrimpont, P., Rouleau, J.L., Wun, C.C., Ciampi, A., Klein, M., Sussex, B., Arnold, J.M., Moye, L., \& Pfeffer, M. (1997). Additive beneficial effects of beta-blockers to angiotensin converting enzyme inhibitors in the Survival and Ventricular Enlargement (SAVE) study. Journal of American College of Cardiology, 29(2), 229-36.

Venturini, F., Romero, M., Toggnoni, G. (1999). Patterns of practice for acute myocardial infarction in a population from ten countries. European Journal of Clinical Pharmacology, 54(11), 877-86.

White, C.M. (1999). Prevention of Sub optimal beta-blocker treatment in patients with myocardial infarction. Annals of Pharmacotherapy, 33(10), 1063-72.

Whitford, D.L. \& Southern A.J. (1994). Audit of secondary prophylaxis after myocardial infarction. British Medical Journal, 309(6964), 1268-9.

Wilkinson, P., Laji, K., Ranjadayalan, K., Parsons, L., \& Timmis, A.D. (1994). Acute myocardial infarction in women: survival analysis in first six months. British Medical Journal, 309(6954), 566-9.

Woods, K.L., Ketley, D., Lowy, A., Agusti, A., Hagn, C., Kala, R. et al. (1998). Beta-blockers and anti-thrombotic treatment for secondary prevention after acute myocardial infarction. European Heart Journal, 19(1), 74-79. 
Yusuf, S., Peto, P., Lewis, J., Collins, R., \& Sleight, P. (1985). Beta blockade during and after myocardial infarction: an overview of the randomized trials. Progress in Cardiovascular Diseases, 27(5), 335-71.

Zeirler, B.K., Meissner, M.H., Cain, K., \& Strandness, D.E. (2002). A survey of physicians' knowledge and management of venous thromboembolism. Vascular and Endovascular Surgery, 36(5), 367-75. 
APPENDICES 


\section{APPENDIX A: CLAIM FILES}

\section{Demographic File}

1. RECIPIENT NUMBER

2. RECIPIENT SOCIAL SECURITY NUMBER

3. DATE OF BIRTH

4. SEX

5. RACE

6. ELIGIBILITY BEGIN DATE

7. ELIGIBILITY END DATE

\section{Hospital / ER File}

1. RECIPIENT NUMBER

2. RECIPIENT SOCIAL SECURITY NUMBER

3. FIRST DATE OF SERVICE

4. LAST DATE OF SERVICE

5. BILLED AMOUNT

6. PAID AMOUNT

7. DRG CODE

8. PROCEDURE CODE

9. DIAGNOSIS CODES

10. HOSPITAL EXTRACT INDICATOR 


\section{Physician File}

1. RECIPIENT NUMBER

2. RECIPIENT SOCIAL SECURITY NUMBER

3. FIRST DATE OF SERVICE

4. LAST DATE OF SERVICE

5. BILLED AMOUNT

6. PAID AMOUNT

7. DRG CODE

8. PROCEDURE CODE

9. DIAGNOSIS CODES

10. PHYSICIAN EXTRACT INDICATOR

\section{Pharmacy File}

1. RECIPIENT NUMBER

2. RECIPIENT SOCIAL SECURITY NUMBER

3. DATE OF PRESCRIPTION FILLED

4. BILLED AMOUNT

5. PAID AMOUNT

6. NDC CODE

7. GENERIC CODE

8. DAYS SUPPLY 


\section{Managed Care File}

1. RECIPIENT SOCIAL SECURITY NUMBER

2. MANAGED CARE BEGIN DATE

3. MANAGED CARE END DATE

\section{Drug File}

1. NATIONAL DRUG CODE

2. DRUG NAME

3. GENERIC CODE

DRG File

1. DRG CODE

2. DRG NAME

\section{Procedure File}

1. PROCEDURE

2. CODE DESCRIPTION

Diagnosis File

1. DIAGNOSIS CODE

2. CODE DESCRIPTION 


\section{APPENDIX B: CODES FOR IDENTIFICATION}

\section{Relative Contraindications to Beta-blocker Therapy Post Acute Myocardial Infarction}

(AMI)

Heart rate less than $60 \mathrm{bpm}$

Systolic arterial pressure less than $100 \mathrm{Hg}$

Moderate or severe left ventricular failure

Signs of peripheral hypoperfusion
Cannot be identified

Cannot be identified

ICD-9 code 428.1

Cannot be identified, Proxy ICD-9

code 443

Second or third degree atrioventricular(AV) block $\quad$ ICD-9 code 426.11, 426.13

PR interval greater than 0.24 second

proxy AV block

Severe Chronic Obstructive Pulmonary Disease

Asthma

Severe peripheral vascular disease

Insulin dependent diabetes mellitus

Beta-blocker intolerance
ICD-9 codes 490, 491.x, 492.x,

494.x, 495.x, and 496

ICD-9 code 493. $x$

CPT-4 code 35456, 35521, 35533,

35546, 35551-58, 35566, 35621,

$35646,35651-61,35666,35700$

ICD-9 code 443

ICD-9 code 250.x 1 and 250.x3

Cannot be identified

\section{Absolute Contraindications to Beta-blocker Therapy Post AMI}

AV block

Bradycardia

Hypotension
ICD-9 code 426.0

ICD-9 code 427.89

ICD-9 code 458.x 


\section{Identifying Codes for Other Conditions Utilized in the Study}

AMI

Diabetes

Hypertension

Congestive heart failure

Coronary artery bypass graft

Percutaneous transluminal coronary

angioplasty

Stroke

Renal dysfunction
ICD-9 code 410. $x$

ICD-9 code 250. $x$

ICD-9 code 401

ICD-9 code 428. $\mathrm{x}$

CPT-4 coded 33517-30, 33533-36,

33542, 33545 DRG-code 107, 109

CPT-4 codes 35470-73

DRG-code 112, 116

ICD-9 code 430-436

ICD-9 code 588.0

\section{Cardiac Conditions}

Essential hypertension

AMI

Acute Ischemia

Angina pectoris

Other Ischemia

Pulmonary heart disease

Disease of Endocardium

Cardiomyopathy

Conduction Disorders

Cardiac Dysrhythmias

Shock
ICD-9 code 401. $\mathrm{x}$

ICD-9 code 410.x

ICD-9 code 411.x

ICD-9 code 413. $x$

ICD-9 code 414.X

ICD-9 code 416. $\mathrm{x}$

ICD-9 code 424. $x$

ICD-9 code 425. $x$

ICD-9 code 426. $x$

ICD-9 code 427. $x$

ICD-9 code 785.5-785.51 


\section{Medications}

Aspirin

Angiotensin converting enzyme inhibitor

Benazepril, Captopril, Cilazapril, Enalapril, Enalaprilat,

Fosinopril, Lisinopril, Moexipril, Perindopril, Quinapril,

Ramipril, Trandolapril

Calcium Channel blockers Bepridil, Diltiazem, Felodipine, Flunarizine, Isradipine,

Nicardipine, Nifedipine, Nimodipine, Verapamil

Loop diuretics

Bumetanide, Ethacrynic Acid, Furosemide

Digoxin

Beta blockers

Acebutolol, Atenolol, Betaxolol, Bisoprolol, Carteolol,

Labetalol, Metoprolol, Nadolol, Oxprenolol, Penbutolol,

Pindolol, Propranolol, Sotalol, Timolol

Lipid Lowering Drugs

Atorvastatin, Cerivastatin, Fluvastatin, Pravastatin,

Simvastatin, Lovastatin

Nitroglycerine

Antiarrhythmics

Amiodarone, Flecainide, Mexeletine, Procanamide,

Propafenone 
COVER LETTERS, STUDY SURVEY AND NON-RESPONSE SURVEY 


\section{APPENDIX C: FIRST COVER LETTER}

January 6, 2003

\section{Dear Doctor,}

Acute Myocardial Infarction (AMI) is the number one killer among both men and women in the United States. It is an area of high expenditures in the health care system. Thus, therapeutic interventions that reduce morbidity, mortality, as well as prevention strategies for AMI are extremely important and can have a significant impact on the quality and cost of patient care in the US health system.

Therapy with beta-blockers and ACE-inhibitors has been investigated in numerous clinical trials for reducing mortality following an AMI. The objective of this study is to identify current opinions and beliefs among WV physicians about the use of secondary preventive agents for long-term therapy in patients who have had an AMI. This study is part of a doctoral research project and is being undertaken by West Virginia University, School of Pharmacy. Information obtained from this study will be useful in developing educational strategies for use of secondary preventive medications in postAMI patients.

We hope that you will choose to participate by completing the attached questionnaire that asks about your opinions and beliefs regarding use of secondary preventive agents in post-AMI patients. To assure confidentiality, your responses will be coded and your name will not appear in any data analysis or study reports. Your participation in this study is voluntary. Although we hope that you will answer all of the questions, you do not have to answer any question that makes you uncomfortable.

Your response will provide valuable information and is critical to the results of the study. Thank you for taking a few minutes to complete the survey. Please return it in the enclosed postage-paid envelope. If you have any questions, please do not hesitate to contact Ancilla Fernandes at (304) 293-6991 or Dr. Suresh Madhavan at (304) 293-1652.

Thank You.

Sincerely,

Ancilla Fernandes, M.S.

Ph.D. Candidate
Suresh Madhavan, M.B.A, Ph.D. Professor and Chairperson 


\section{APPENDIX D: SECOND COVER LETTER}

January 20, 2003

Dear Doctor,

About two weeks ago, we sent you a survey asking your opinions and beliefs related to use of secondary preventive agents in post-AMI patients. We have not yet received your completed survey.

We understand that you are busy or may not have received the survey. However, your responses are extremely important to us, and the information obtained for the study will be very useful to develop educational strategies for enhancing use of secondary preventive agents in post-AMI patients. Therefore, we are sending you another copy of the survey and would appreciate it if you could take a few minutes to complete and return it in the enclosed postage-paid envelope. If you have responded, please disregard this letter and accept our gratitude for your time and effort.

This study is part of a doctoral research project and is being undertaken by West Virginia University, School of Pharmacy. Your participation in this study is voluntary. To assure confidentiality, your responses will be coded and your name will not appear in any data analysis or study reports. Although we hope that you could answer all of the questions, you do not have to answer any question that makes you uncomfortable.

Thank you for your participation. If you have any questions or concerns, please do not hesitate to contact Ancilla Fernandes at (304) 293-6991 or Dr. Suresh Madhavan at (304) 293-1652.

Thanks you.

Sincerely,

Ancilla Fernandes, M.S.

Ph.D. Candidate
Suresh Madhavan, M.B.A, Ph.D. Professor and Chairperson 


\section{APPENDIX E: THIRD COVER LETTER}

February 3, 2003

\section{Dear Doctor,}

About two weeks ago, we made our second attempt to reach you regarding our study, asking your opinions and beliefs related to use of secondary preventive agents in post-AMI patients. We have not yet received your completed survey.

We have undertaken this study because it is our belief that your opinions should be taken into account for developing educational strategies for enhancing use of secondary preventive agents in post-AMI patients.

We are writing to you again to emphasize the significance of your response and how important your opinions are in understanding patient care for AMI patients. We have enclosed a questionnaire just in case your earlier copies have been misplaced. We would appreciate it if you could take a few minutes to complete and return it in the enclosed postage-paid envelope. If you have responded, please disregard this letter and accept our gratitude for your time and effort.

This study is part of a doctoral research project and is being undertaken by West Virginia University, School of Pharmacy. Your participation in this study is voluntary. To assure confidentiality, your responses will be coded and your name will not appear in any data analysis or study reports. Although we hope that you could answer all of the questions, you do not have to answer any question that makes you uncomfortable.

Thank you for your participation. If you have any questions or concerns, please do not hesitate to contact Ancilla Fernandes at (304) 293-6991 or Dr. Suresh Madhavan at (304) 293-1652.

Thanks you.

Sincerely,

Ancilla Fernandes, M.S.

Ph.D. Candidate
Suresh Madhavan, M.B.A, Ph.D.

Professor and Chairperson 
APPENDIX F: STUDY SURVEY 


\section{Opinions and Beliefs about Long-term Secondary Preventive Agents following an Acute Myocardial Infarction}

(AMI)

INSTRUCTIONS: Your professional opinions and beliefs are extremely important for understanding the use of secondary preventive agents following an AMI. Please answer the questions carefully. Your response will be kept absolutely confidential.

\section{SECTION A}

1. Of the following medications, which according to you are the important secondary preventive agents following an AMI:(Check all that apply)
Aspirin
Angiotensin Converting Enzyme (ACE) inhibitors
Digitalis
Anti-arrhythymics
Calcium Channel blockers
Nitroglycerin
Beta-blockers
Lipid-lowering drugs
Anticoagulants
Magnesium
Others, please specify

2. How likely are you, on a scale from 1 to 5 where 1 is least likely and 5 is most likely, to prescribe these medications for secondary prevention following an AMI:

\section{Please answer this question by using:}

\section{Least likely}

\section{5 \\ Uncertain Most likely \\ Enter response (any number from 1 to 5)}

1. Aspirin

2. Angiotensin Converting Enzyme (ACE) inhibitors

3. Digitalis

4. Anti-arrhythymics

5. Calcium Channel blockers

6. Nitroglycerin

7. Beta-blockers

8. Magnesium

9. Lipid-lowering drugs

10. Anticoagulants

11. Others

3. A) Of the last 10 new AMI/post-AMI patients that you treated, you prescribed a beta-blocker as long- term therapy for secondary prevention in....( Please circle the appropriate number)

$\begin{array}{llllllllll}1 & 2 & 3 & 4 & 5 & 6 & 7 & 8 & 9 & 10\end{array}$

B) If your answer for the previous question is less than 10 please specify the reasons for not prescribing beta-blockers in these patients:

4. A) Of the last 10 new AMI/post-AMI patients that you treated, you prescribed a ACE inhibitor as long-term therapy for secondary prevention in....( Please circle the appropriate number)

$\begin{array}{llllllllll}1 & 2 & 3 & 4 & 5 & 6 & 7 & 8 & 9 & 10\end{array}$

B) If your answer for the previous question is less than 10 please specify the reasons for not prescribing ACE inhibitors in these patients: 


\section{SECTION B}

Clinical trials have consistently demonstrated lower mortality in AMI patients when they are treated with betablockers and ACE inhibitors. However, there are certain conditions in the presence of which beta-blockers and/or ACE inhibitors are not beneficial and can be harmful.

Given the normal side effects of beta-blockers (fatigue, sexual dysfunction, nightmares and difficulty to detect hypoglycemia in diabetics) and ACE inhibitors (dry cough, diarrhea, fatigue, dizziness) how likely are you, on a scale from 1 to 5 where 1 is least likely and 5 is most likely, to prescribe these for long-term use for secondary prevention in AMI patients with the following attributes:

Please answer this question by using:

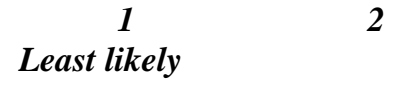

Enter response (any number from 1 to 5)

1. Age $>50$ years

2. Age $\leq 50$ years

3. Large or anterior AMI

4. Small infarction

5. Previous infarction

6. $\mathrm{EF}<40 \%$

7. History of Chronic Heart Failure

8. Had an angioplasty

9. Had by-pass surgery

10. Stroke

11. Hypotension (systolic pressure $<90 \mathrm{Hg}$ )

12. Hypertension (systolic pressure $>140 \mathrm{Hg}$ )

13. Systolic blood pressure $<100 \mathrm{Hg}$

14. Bradycardia (heart rate $<50 \mathrm{bpm}$ )

15. Heart rate $<60 \mathrm{bpm}$

16. Left Venticular (LV) failure

17. Complex Ventricular ectopy

18. First degree Atrio-Ventricular (AV) block

19. Second degree AV block

20. Third degree AV block

21. Peripheral vascular disease

22. Chronic Obstructive Pulmonary Disease (COPD)

23. Asthma

24. Insulin dependent diabetes mellitus
Beta-blockers

\section{Most likely}




\section{SECTION C}

Patient conditions in the presence of which medications such as beta-blockers and ACE inhibitors are not beneficial are divided into absolute contraindications (i.e. medication should not be used), and relative contraindications (i.e. medication can be used if patient is monitored). There are other conditions that are not contraindications (i.e. medication can be used without concern).

Which of the following conditions in your opinion are - absolute contraindications (AC), relative contraindications (RC), or are not contraindications (NC) for long-term use of these drugs (beta-blockers and ACE inhibitors) in AMI patients. Please answer this question by marking $(\mathrm{X})$ in the box that represents your answer.

Patient conditions:

1. Hypotension (systolic pressure $<90 \mathrm{Hg}$ )

2. Systolic blood pressure $<100 \mathrm{Hg}$

3. Hypertension (systolic pressure $>140 \mathrm{Hg}$ )

4. Heart rate $<60 \mathrm{bpm}$

5. Bradycardia (heart rate $<50 \mathrm{bpm}$ )

6. Peripheral vascular disease

7. Peripheral hypoperfusion

8. $\mathrm{EF}<40 \%$

9. First degree Atrio-Ventricular (AV) block

10. Second degree AV block

11. Third degree AV block

12. Chronic Obstructive Pulmonary Disease (COPD)

13. Asthma

14. Non-insulin dependent diabetes mellitus

15. Insulin dependent diabetes mellitus

16. PR interval on Electrocardiogram $(E C G)>0.24$ second

17. Stroke

18. Beta-blocker intolerance

19. ACE-inhibitor intolerance

\section{Beta-blockers}

AC RC NC

$1 \quad 1 \quad 1$

$1-1 \quad 1$

$1 \quad 1 \quad 1$

$1 \quad 1 \quad 1$

$1 \quad 1 \quad 1$

$1 \quad 1 \quad 1$

$\begin{array}{lll}1 & 1 & 1\end{array}$

$\begin{array}{lll}1 & 1 & 1\end{array}$

$1 \quad 1 \quad 1$

1

$1 \quad 1 \quad 1$

$\begin{array}{lll}1 & 1 & 1\end{array}$

$1 \quad 1 \quad 1$

$1 \quad 1 \quad 1$

$1 \quad 1 \quad 1$

$1 \quad 1 \quad 1$

$\begin{array}{lll}1 & 1 & 1\end{array}$

$1 \quad 1 \quad 1$

1
ACE inhibitors

AC RC NC

$1 \quad 1 \quad 1$

$1 \quad 1$

$1 \quad 1 \quad 1$

$\begin{array}{lll}1 & 1 & 1\end{array}$

$\begin{array}{lll}1 & 1 & 1\end{array}$

$\begin{array}{lll}1 & 1 & 1\end{array}$

$1 \quad 1 \quad 1$

$\begin{array}{lll}1 & 1 & 1\end{array}$

$1 \quad 11$

$1 \quad 1 \quad 1$

$1 \quad 1 \quad 1$

$\begin{array}{lll}1 & 1 & 1\end{array}$

$\begin{array}{lll}1 & 1 & 1\end{array}$

$1 \quad 1 \quad 1$

$1 \quad 1 \quad 1$

$1 \quad 11$

$\begin{array}{lll}1 & 1 & 1\end{array}$

$\begin{array}{lll}1 & 1 & 1 \\ 1 & 1 & 1\end{array}$




\section{SECTION D}

\section{A few questions about you and your primary site of practice.}

1. Age: __ Years __ Months

2. Gender: Male Female

3. Specialty or Primary Area of Practice:

4. Year of board certification in this specialty/primary area of practice:

5. Your primary practice site is:

$\begin{array}{ll}\text { Hospital based } & \text { (go to question 6) } \\ \text { University-affiliated Hospital } & \text { (go to question 6) } \\ \text { Solo, office-based } & \text { (go to question 8) } \\ \text { Group, office-based } & \text { (go to question 8) }\end{array}$

Others, please specify (go to question 8)

6. The hospital where you practice is: Check all that apply

$\begin{array}{ll}\text { Rural } & \text { Government/Non-Federal } \\ \text { Urban } & \text { Government/Federal } \\ \text { Sub-urban } & \text { Private Not-For-Profit } \\ & \text { Private Investor Owned }\end{array}$

Others, please specify

7. Number of beds at this hospital:

8. Average number of new AMI patients you see per month:

9. Average number of repeat AMI patients you see per month:

\section{COMMENTS}

If there is anything else that you would like to tell us about your beliefs or opinions with using beta-blockers and ACEinhibitors as secondary preventive agents after an AMI, please use this space to provide your comments.

\section{THANK YOU}

Please return the complete survey in the enclosed business reply envelope. 


\section{APPENDIX G: NON-RESPONSE SURVEY}

February 17, 2003

\section{Dear Doctor,}

During the past two months you may have received two-three mailings of a questionnaire regarding your opinions and beliefs for use of secondary preventive agents in post-Acute Myocardial Infarction (AMI) patients. We realize that you have been very busy and have chosen not to answer the survey or did not have the time. Since your view is extremely important to us, we would like to know your reason for not responding and obtain some key information for the study. This study is part of a doctoral research project and is being undertaken by West Virginia University, School of Pharmacy. Your participation in this study is voluntary. To assure confidentiality, your responses will be coded and your name will not appear in any data analysis or study reports. Although we hope that you could answer all of the questions, you do not have to answer any question that makes you uncomfortable. Please answer the few questions below and mail it to us in the business reply envelope provided. If you have any questions or concerns, please do not hesitate to contact us at (304) 293-6991 or (304) 293-1652.

Sincerely,

Ancilla Fernandes, M.S.

Suresh Madhavan, M.B.A, Ph.D.

Ph.D. Candidate

Professor and Chairperson

\section{Q1. I did not respond to the survey because:}

Did not have enough time to complete it

Did not receive it

Forgot about the survey

Survey was too long

Survey was misplaced

Don't like to respond to surveys

No incentive to complete it

Not enough information about benefits

Not interested in such studies

Topic was irrelevant

Others (Please specify)

\section{Q2. Demographics:}

1. Age:

Years

Months

2. Gender: Male Female

3. Specialty or Area of Practice:

4. Year of board certification in this specialty/area of practice:

5. Your primary practice site is:

Solo, office-based

Group, office-based

Hospital based

University affiliated-Hospital based

6. Average number of new AMI patients you see per month:

7. Average number of repeat AMI patients you see per month: 


\section{CURRICULUM VITAE}

ANCILLA W. FERNANDES

1056 Van Voorhis Road, Apt \# K308, Morgantown, WV 26505

Home: (304) 293-2558. Work: (304) 293-8194, 0755. Fax: (304) 293-2529

E-mail: afernandes@ hsc.wvu.edu

\section{AREAS OF RESEARCH INTEREST}

Health outcomes research, Pharmacoepidemiology, Marketing and Disease management

\section{ACADEMIC TRAINING \& QUALIFICATIONS}

2000- present

Doctoral Candidate (Ph.D.) in Pharmaceutical Systems and Policy, School of Pharmacy, West Virginia University (WVU), Morgantown, WV.

(Expected date of completion June 2003).

Dissertation Title: Evaluating Utilization of Beta-Blockers as Secondary Prevention for Post Myocardial Infarction in a Medicaid Population. Major Area: Health Outcomes and Pharmacoepidemiology Current GPA: 3.90

1997-2000

Master of Science (M.S.) in Pharmaceutical Systems and Policy, School of Pharmacy, West Virginia University (WVU), Morgantown, WV.

Thesis Title: Evaluating Diagnostic and Treatment Modalities in the Management of Benign Prostatic Hyperplasia in the Veterans

Administration Population.

Major Area: Health Services and Outcomes Research GPA: 3.88

1998-1999

Certificate in Health Care Administration

Department of Public Administration

West Virginia University (WVU), Morgantown, WV.

1993-1997

Bachelor's in Pharmaceutical Sciences (B. Pharm.)

Bombay College of Pharmacy,

University of Mumbai,

Bombay, India. 


\section{PROFESSIONAL EXPERIENCE}

1999 - Present $\quad$ Graduate Research Assistant, Office of Drug Abuse and Intervention Studies, Prevention Research Center, Department of Community Medicine, WVU.

Data management, statistical analysis and development of evaluation measures for Not-On-Tobacco projects conducted among high school students.

2002-2003

Summer 2001

$2000-2001$

Summer 2000

Guest Lecturer, Department of Pharmaceutical Systems and Policy, School of Pharmacy, West Virginia University.

Outcomes Assessment and Quality Improvement. Responsibilities included lecturing and preparing handouts on topics in pharmacoeconomics such as cost effectiveness analyses. Health promotion and Disease Prevention. Responsibilities included lecturing and preparing handouts on the topic of a successful community intervention.

Internship, Eli Lilly and Company, US Health Outcomes and Evaluation Group.

Evaluating pharmaceutical utilization for type 2 diabetics in PCS database.

Part-time Graduate Research Assistant, The economic burden of Barrett's esophagus and esophageal cancer in the West Virginia Medicaid population (Funded by GD Searle and Company).

Evaluating epidemiology of Barrett's esophagus to esophageal cancer in the state Medicaid.

Graduate Research Assistant, Pharmacy Immunization Project, Department of Pharmaceutical Systems and Policy, School of Pharmacy, West Virginia University.

Assessing predictors of immunization in rural adults and rural seniors.

$1997-1999$

Graduate Teaching Assistant, Department of Pharmaceutical Systems and Policy, School of Pharmacy, West Virginia University.

Pharmacy Management: Responsibilities included conducting recitations on financial case studies and problem solving activities, formulating and evaluating assignments, and group discussions.

Pharmacy Care Lab \& Patient Health Education: Responsibilities included conducting communication skills activities, evaluating student verbal and non-verbal patient consultation skills, and grading group presentations.

Summer 1996 Internship, Fulford (India) Ltd., an affiliate of Schering Plough. Underwent practical training in various departments. 


\section{RESEARCH PRESENTATIONS}

Kamal K, Kalsekar I, Fernandes A, Horn K, \& Dino G. Stage of Change and Smoking Cessation Outcomes in Adolescents. Poster presentation at the 2002 National Conference on Tobacco or Health (November, 2002).

Massey CJ, Horn KA, Dino GA, McCraken A, Goldcamp J, \& Fernandes AW. Recruiting Techniques for School-Based Interventions. Podium presentation at the 75th meeting of the American School Health Association, Albuquerque (by CJ Massey) (November, 2001).

Fernandes AW, Kalsekar I, Horn KA, Dino GA, \& Massey CJ. Adolescent Nicotine Dependence and Smoking Cessation Outcomes. Poster presentation at the $9^{\text {th }}$ Society of Prevention Research meeting (May, 2001).

Horn KA, Dino GA, Goldamp J, Fernandes AW, Kalsekar I, \& Massey CJ. A Two-Year Efficacy of Not On Tobacco. Poster presentation at the $9^{\text {th }}$ Society of Prevention Research meeting (May, 2001).

Fernandes AW, Amonkar MM, Madhavan SS, \& Nseyo UO. Benign Prostatic Hyperplasia Management in the Veterans Administration Population: A National Survey of Urologists. Poster presentation at the $148^{\text {th }}$ APhA Annual meeting (March, 2001).

Dino GA, Horn KA, Maniar SD, Kemp-Rye L, Fernandes AW, \& Massey CJ. The Relationship between Mental Health and Smoking Cessation among Adolescents. Poster presentation at $108^{\text {th }}$ APA meeting (August, 2000).

Fernandes AW, Amonkar MM, Madhavan SS, \& Nseyo UO. Matching VA Urologists Preferences in the Management of Benign Prostatic Hyperplasia with Clinical Guidelines. Poster presentation at $5^{\text {th }}$ ISPOR Annual International Meeting (May, 2000).

Fernandes AW, Amonkar MM, Madhavan SS, \& Nseyo UO. Evaluating Diagnostic and Treatment Modalities in the Management of Benign Prostatic Hyperplasia in the Veterans Administration Population. Poster presentation at the $146^{\text {th }}$ APhA Annual meeting (March, 1999).

\section{RESEARCH PUBLICATIONS}

Dino G, Kamal K, Horn K, Kalsekar I, \& Fernandes A (in press). Stages of change and smoking cessation outcomes among adolescents. Addiction Behavior.

Madhavan SS, Borker RD, Fernandes AW, Amonkar MM, \& Rosenbluth SA (in press). Assessing predictors of influenza and pneumonia vaccination in rural senior adults. Journal of Health and Social Policy.

Horn KA, Fernandes AW, Massey CJ, Kalsekar I, \& Dino GA. Adolescent nicotine dependence and smoking cessation outcomes. Addiction Behavior (June 2003). 
Madhavan SS, Rosenbluth SA, Amonkar MM, Fernandes AW, \& Borker RD. Predictors of influenza and pneumonia vaccinations in rural adults 18 to 64 years of age. Journal of Healthcare for the Poor and Undeserved (February 2003).

Horn KA, Dino GA, Goldcamp J, Fernandes AW, Kalsekar I, \& Massey CJ. A two-year efficacy study of Not On Tobacco in FL (1999-2000). Preventive Medicine (October 2001).

Dino GA, Horn KA, Goldcamp J, Maniar SD, Fernandes AW, \& Massey CJ. State-wide demonstration of Not On Tobacco: A gender-sensitive teen smoking cessation program. Journal of School Nursing (April 2001).

Fernandes AW, Amonkar MM, Madhavan SS, \& Nseyo UO. Assessing practice preferences of urologists in the management of BPH in the Veterans Administration program. Current Therapeutic Research (February 2001).

Horn K, Dino G, Fernandes A, \& Kalsekar I (under review). Appalachian teen smokers: NotOn-Tobacco 15 months later. American Journal of Public Health.

\section{RELEVANT COURSE WORK}

Epidemiology, Pharmacoeconomic Analysis, Outcomes Research, Secondary Data Analysis, Managed Care, Statistics (univariate, multiple regression, multivariate analysis), Health Services Marketing, Consumer Behavior, and Health Care Administration, Econometrics.

\section{COMPUTER SKILLS}

SPSS, SAS, Survey Pro, TreeAge, MS Word, MS Excel, MS PowerPoint, Corel WordPerfect, Atrisk (Monte-Carlo simulation), AMOS, and E-views.

\section{AWARDS AND HONORS}

2002

2001

1998
Vice-President WVU-ISPOR (ISPOR Student Chapter)

Graduate Research Award (School of Pharmacy, WVU)

Rho Chi Pharmacy Honor Society

\section{PROFESSIONAL MEMBERSHIP}

1999- Present International Society for Pharmacoeconomics and Outcomes Research 1999- Present American Pharmaceutical Association

\section{REVIEWER}

Journal of American Pharmaceutical Association 